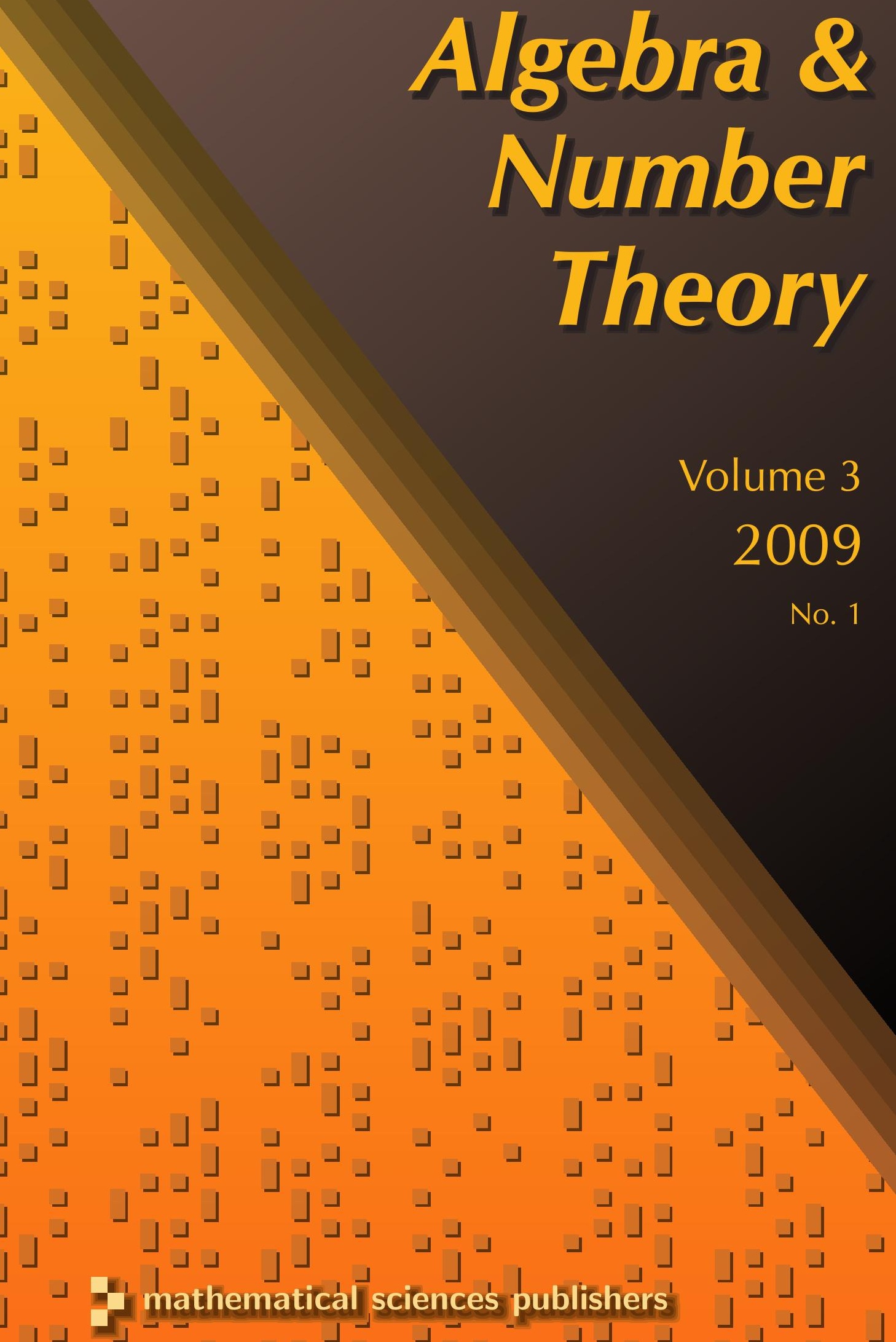




\section{Algebra \& Number Theory}

www.jant.org

\section{EDITORS}

\author{
MANAGING EDITOR \\ Bjorn Poonen \\ University of California \\ Berkeley, USA
}

\author{
EDITORIAL BOARD CHAIR \\ David Eisenbud \\ University of California \\ Berkeley, USA
}

\section{BOARD OF EDITORS}

Georgia Benkart

Dave Benson

Richard E. Borcherds

John H. Coates

J-L. Colliot-Thélène

Brian D. Conrad

Hélène Esnault

Hubert Flenner

Edward Frenkel

Andrew Granville

Joseph Gubeladze

Ehud Hrushovski

Craig Huneke

Mikhail Kapranov

Yujiro Kawamata

János Kollár

Hendrik W. Lenstra

Yuri Manin

Barry Mazur
University of Wisconsin, Madison, USA

University of Aberdeen, Scotland

University of California, Berkeley, USA

University of Cambridge, UK

CNRS, Université Paris-Sud, France

University of Michigan, USA

Universität Duisburg-Essen, Germany

Ruhr-Universität, Germany

University of California, Berkeley, USA

Université de Montréal, Canada

San Francisco State University, USA

Hebrew University, Israel

University of Kansas, USA

Yale University, USA

University of Tokyo, Japan

Princeton University, USA

Universiteit Leiden, The Netherlands

Northwestern University, USA

Harvard University, USA
Susan Montgomery

Shigefumi Mori

Andrei Okounkov

Raman Parimala

Victor Reiner

Karl Rubin

Peter Sarnak

Michael Singer

Ronald Solomon

Vasudevan Srinivas

J. Toby Stafford

Bernd Sturmfels

Richard Taylor

Ravi Vakil

Michel van den Bergh

Marie-France Vignéras

Kei-Ichi Watanabe

Andrei Zelevinsky

Efim Zelmanov
University of Southern California, USA

RIMS, Kyoto University, Japan

Princeton University, USA

Emory University, USA

University of Minnesota, USA

University of California, Irvine, USA

Princeton University, USA

North Carolina State University, USA

Ohio State University, USA

Tata Inst. of Fund. Research, India

University of Michigan, USA

University of California, Berkeley, USA

Harvard University, USA

Stanford University, USA

Hasselt University, Belgium

Université Paris VII, France

Nihon University, Japan

Northeastern University, USA

University of California, San Diego, USA

\section{PRODUCTION}

ant@mathscipub.org

Paulo Ney de Souza, Production Manager

Silvio Levy, Senior Production Editor

See inside back cover or www.jant.org for submission instructions.

Regular subscription rate for 2009: \$200.00 a year (\$140.00 electronic only).

Subscriptions, requests for back issues from the last three years and changes of subscribers address should be sent to Mathematical Sciences Publishers, Department of Mathematics, University of California, Berkeley, CA 94720-3840, USA.

Algebra \& Number Theory, ISSN 1937-0652, at Mathematical Sciences Publishers, Department of Mathematics, University of California, Berkeley, CA 94720-3840 is published continuously online. Periodical rate postage paid at Berkeley, CA 94704, and additional mailing offices.

\section{PUBLISHED BY \\ 7. mathematical sciences publishers \\ http://www.mathscipub.org}

A NON-PROFIT CORPORATION

Typeset in IATEX

Copyright $\odot 2009$ by Mathematical Sciences Publishers 


\title{
On the additive dilogarithm
}

\author{
Sinan Ünver
}

Let $k$ be a field of characteristic zero, and let $k[\varepsilon]_{n}:=k[\varepsilon] /\left(\varepsilon^{n}\right)$. We construct an additive dilogarithm $\mathrm{Li}_{2, n}: B_{2}\left(k[\varepsilon]_{n}\right) \rightarrow k^{\oplus(n-1)}$, where $B_{2}$ is the Bloch group which is crucial in studying weight two motivic cohomology. We use this construction to show that the Bloch complex of $k[\varepsilon]_{n}$ has cohomology groups expressed in terms of the K-groups $K_{(\cdot)}\left(k[\varepsilon]_{n}\right)$ as expected. Finally we compare this construction to the construction of the additive dilogarithm by Bloch and Esnault defined on the complex $T_{n} \mathbb{Q}(2)(k)$.

\section{Introduction}

1.1. For any scheme $S$ one expects a category $\mu_{S}$ of motivic (perverse) sheaves over $S$, which should be an abelian tensor category that satisfies all the formalism of mixed sheaf theory [Berlinson 1987, 5.10]. The Tate sheaves $\mathbb{Z}_{\mathcal{M}}(n)$ should play a special role. Namely, letting

$$
H^{i}\left(S, \mathbb{Z}_{\mathcal{M}}(n)\right):=\operatorname{Ext}_{\mathcal{M}_{S}}^{i}\left(\mathbb{Z}_{\mathcal{M}}(0), \mathbb{Z}_{\mathcal{M}}(n)\right),
$$

the Chern character map

$$
K_{2 n-i}(S)_{\mathbb{Q}}^{(n)} \rightarrow H^{i}\left(S, \mathbb{Q}_{\mu}(n)\right)
$$

from the $n$-th graded piece of Quillen's K-theory tensored with $\mathbb{Q}$, defined as the $k^{n}$-eigenspace for the $k$-th Adams operator (Remark 3.1.2), to motivic cohomology of weight $n$ should be an isomorphism when $S$ is regular (loc. cit.). Since $M_{S}$ is to have realizations corresponding to various cohomology theories, the regulator map

$$
K_{2 n-i}(S)_{\mathbb{Q}}^{(n)} \rightarrow H^{i}\left(S, \mathbb{Q}_{M}(n)\right) \rightarrow H_{*}^{i}\left(S, \mathbb{Q}_{*}(n)\right),
$$

where $*$ is the relevant realization, gives arithmetically important information.

The complexes $\underline{\mathrm{RHom}}_{\mathrm{Zar}}\left(\mathbb{Z}_{\mathcal{M}}(0), \mathbb{Z}_{\mathcal{M}}(n)\right)$ of sheaves on the Zariski site should have the property that $H^{i}\left(S_{\mathrm{Zar}}, \mathrm{RHom}_{\mathrm{Zar}}\left(\mathbb{Z}_{M}(0), \mathbb{Z}_{M}(n)\right)\right)=H^{i}\left(S, \mathbb{Z}_{M}(n)\right)$. Hence the motivic cohomology of $S$ of weight $n$ could be computed as the hypercohomology of a complex of sheaves on $S_{\mathrm{Zar}}$.

MSC2000: 11G55.

Keywords: polylogarithms, additive polylogarithms, mixed Tate motives, Hilbert's 3rd problem. 
Recently Voevodsky and others have made progress in motivic cohomology [Mazza et al. 2006]. If $S=\operatorname{Spec}(k)$, where $k$ is a field of characteristic zero, Voevodsky constructs a triangulated category $D M_{\mathrm{Nis}}^{\mathrm{eff},-}(k)$ [loc. cit., Chapter 14] and a complex of sheaves $\mathbb{Z}(n)$ on the big Zariski site over $k$, which should be isomorphic to the hypothetical $\underline{\mathrm{RHom}}_{\mathrm{Zar}}\left(\mathbb{Z}_{\mathcal{M}}(0), \mathbb{Z}_{\mathcal{M}}(n)\right)$ above, such that for any smooth scheme $X$ over $k$,

$$
H^{i}\left(X_{\mathrm{Zar}}, \underline{\mathbb{Z}}(n)\right) \simeq \mathrm{Ext}_{D M_{\mathrm{Nis}}^{\mathrm{eff},-}}^{\mathrm{ef}}(M(X), \underline{\mathbb{Z}}(n))
$$

(see [loc. cit., 14.16]), where $M(X)$ is the motive of $X$ [loc. cit., Definition 14.1]. Since $\underline{\mathbb{Z}}(n)$ and Bloch's complex of algebraic cycles of codimension $n$ are isomorphic [loc. cit., Chapter 19], the Bloch-Grothendieck-Riemann-Roch theorem [Bloch 1986] implies that the hypercohomology of $\underline{\mathbb{Q}}(n)$ on $X_{\text {Zar }}$ is expressed in terms of the K-groups of $X$ as above:

$$
K_{2 n-i}(X)_{\mathbb{Q}}^{(n)} \simeq H^{i}\left(X_{\text {Zar }}, \underline{\mathbb{Q}}(n)\right) .
$$

In order to study the motivic cohomology of $S$, it would be sufficient to restrict to a subcategory of $\mu_{S}$. Let $M \mathcal{T} M_{S}$ denote the smallest full subcategory of $\mu_{S}$ that contains the Tate motives and is closed under extensions. Then $H^{i}\left(S, \mathbb{Q}_{M}(n)\right) \simeq$ $\operatorname{Ext}_{\mathcal{M}_{S}}^{i}\left(\mathbb{Q}_{\mu}(0), \mathbb{Q}_{\mu}(n)\right)=\operatorname{Ext}_{\mathcal{M} \mathcal{T}_{M_{S}}}^{i}\left(\left(\mathbb{Q}_{\mathcal{M}}(0), \mathbb{Q}_{\mu}(n)\right)\right.$. The category $\mathcal{M}_{\mathcal{T}} M_{S}$ would be simpler than $M_{S}$. In fact for $S=\operatorname{Spec}(k)$, where $k$ is a number field, Deligne and Goncharov [2005] have constructed a candidate for $\mathcal{M} \mathcal{T} M_{S}$ as a tannakian category, using $D M_{\mathrm{Nis}}^{\mathrm{eff},-}$.

It is natural to expect that $\mathcal{M} \mathcal{T} \mathcal{M}_{S}$ can be constructed by using only the relative cohomologies of hyperplane arrangements and in turn that motivic cohomology can be computed using complexes of linear algebraic objects [Beřlinson et al. 1990], rather than all algebraic cycles. Special degenerate configurations of hyperplanes, called the polylogarithmic configurations [Beillinson et al. 1990; Goncharov 1995], act as building blocks for all configurations and thus play a special role in describing motivic cohomology.

Using the relations satisfied by the polylogarithmic configurations, Goncharov defines a complex $\Gamma_{k}(n)_{\mathbb{Q}}$ by

$$
\mathscr{B}_{n}(k) \rightarrow \mathscr{P}_{n-1}(k) \otimes k_{\mathbb{Q}}^{\times} \rightarrow \mathscr{B}_{n-2}(k) \otimes \bigwedge^{2} k_{\mathbb{Q}}^{\times} \rightarrow \cdots \rightarrow \mathscr{B}_{2}(k) \otimes \bigwedge^{n-2} k_{\mathbb{Q}}^{\times} \rightarrow \bigwedge^{n} k_{\mathbb{Q}}^{\times},
$$

which he conjectures can be used to compute the motivic cohomology of weight $n$ [Goncharov 1995, Conjectures A and 1.17].

If $k=\mathbb{C}$, integration over the polylogarithmic configurations can be used to define a map $\mathbb{Q}\left[\mathbb{P}^{1}(\mathbb{C})\right] \rightarrow \mathbb{R}$, the single-valued real analytic version of the $n$-th polylogarithmic function [Goncharov 1995, 1.0], which factors through the projection $\mathbb{Q}\left[\mathbb{P}^{1}(\mathbb{C})\right] \rightarrow \mathscr{B}_{n}(\mathbb{C})$ (loc. cit.) to give $\mathscr{L}_{n}: \mathscr{B}_{n}(\mathbb{C}) \rightarrow \mathbb{R}$, the $n$-th polylogarithm 
that is expected to induce the regulator $K_{2 n-1}(\mathbb{C})_{\mathbb{Q}}^{(n)} \simeq H^{1}\left(\operatorname{Spec}(\mathbb{C}), \mathbb{Q}_{\mu}(n)\right) \rightarrow$ $\mathscr{B}_{n}(\mathbb{C}) \rightarrow \mathbb{R}$ [Goncharov 1995, page 224].

For a general field $k$, one cannot expect a polylogarithm on $\mathscr{B}_{n}(k)$. However, through his interpretation of hyperbolic scissor congruence groups in terms of mixed Tate motives, Goncharov expected that there should be an infinitesimal polylogarithmic function that acts like a regulator map on $K_{2 n-1}\left(k[\varepsilon]_{2},(\varepsilon)\right)^{(n)}$, for any field $k$ of characteristic 0 [Goncharov 1999, pages 616-617; 2004], where $k[\varepsilon]_{m}:=k[\varepsilon] /\left(\varepsilon^{m}\right)$. In our notation, assuming the existence of mixed Tate motives and the complex $\Gamma_{n}$ over the dual numbers, this translates to the existence of a map

$$
\mathscr{B}_{n}\left(k[\varepsilon]_{2}\right) / \mathscr{B}_{n}(k) \rightarrow k
$$

that, when composed with $K_{2 n-1}\left(k[\varepsilon]_{2},(\varepsilon)\right)^{(n)} \rightarrow \mathscr{B}_{n}\left(k[\varepsilon]_{2}\right) / \mathscr{B}_{n}(k)$, gives an isomorphism. The map (1.1.3) is to be an analogue of both the volume map for euclidean scissor congruence groups and of polylogarithms.

In this paper we are interested in this question for weight two. Next we give details about this case.

1.2. Let $A$ be an artinian local ring and $I$ an ideal of $A$. In the rest of the paper, when we refer to weight two (rational) motivic cohomology of $A$ relative to $I$, what we mean are the groups $K_{3}(A, I)_{\mathbb{Q}}^{(2)}$ and $K_{2}(A, I)_{\mathbb{Q}}^{(2)}$ and not to the Voevodsky motivic cohomology groups in Section 1.1, which were there only to motivate the main results of this paper. This common abuse of notions is partly justified by the expected Chern character isomorphism (1.1.1), which is known to be true when $A$ is a field (1.1.2).

Let $k$ be an algebraically closed field of characteristic 0 , let $S$ the semilocal ring of rational functions on $\mathbb{A}_{k}^{1}$ that are regular on $\{0,1\}$, and let $J$ the Jacobson radical of $S$.

The first complex computing the weight two motivic cohomology is constructed by Bloch as follows. Localizing $\mathbb{A}_{k}^{1}$ away from 0 and 1 gives an exact sequence

$$
0 \rightarrow K_{3}(k)^{(2)} \rightarrow K_{2}(S, J) \stackrel{\varphi}{\longrightarrow} \bigoplus_{x \in k^{\times} \backslash\{1\}} k^{\times} \rightarrow K_{2}(k) \rightarrow 0
$$

(see [Lichtenbaum 1987, proof of 7.1; Bloch 1977]), where $\varphi$ is the tame symbol map. Let

$$
B(k):=K_{2}(S, J) / \operatorname{im}\left((1+J) \otimes k^{\times}\right),
$$

the part of $K_{2}(S, J)$ that does not come from the products of weight 1 terms. Then $\left(\bigoplus_{x \in k^{\times} \backslash\{1\}} k^{\times}\right) / \varphi\left((1+J) \otimes k^{\times}\right)=k^{\times} \otimes k^{\times}$, and the sequence

$$
0 \rightarrow K_{3}(k)_{\mathbb{Q}}^{(2)} \rightarrow B(k)_{\mathbb{Q}} \rightarrow\left(k^{\times} \otimes k^{\times}\right)_{\mathbb{Q}} \rightarrow K_{2}(k)_{\mathbb{Q}} \rightarrow 0,
$$

remains exact (same references). 
In complete analogy, Bloch and Esnault [2003] define a complex that computes the motivic cohomology of $k[t]_{2}$ relative to the ideal $(t)$ as follows. Let $R$ be the local ring of $\mathbb{A}_{k}^{1}$ at 0 . Then localizing away from 0 on $\mathbb{A}^{1}$ gives the sequence

$$
K_{2}\left(k[t],\left(t^{2}\right)\right) \rightarrow K_{2}\left(R,\left(t^{2}\right)\right) \stackrel{\varphi}{\longrightarrow} \bigoplus_{x \in k^{\times}} k^{\times} \rightarrow K_{1}\left(k[t],\left(t^{2}\right)\right) .
$$

Let $\mathscr{b}$ denote the subgroup generated by the symbols $\langle a, b\rangle \in K_{2}\left(R,\left(t^{2}\right)\right)$ with $a \in\left(t^{2}\right)$ and $b \in k$, and put $T B(k):=K_{2}\left(R,\left(t^{2}\right)\right) / \mathscr{C}$. Then we have $k^{\times} \otimes k=$ $\left(\bigoplus_{x \in k^{\times}} k^{\times}\right) / \varphi(\mathscr{C})$ and an exact sequence

$$
0 \rightarrow K_{2}\left(k[t],\left(t^{2}\right)\right)^{(2)} \rightarrow T B(k) \rightarrow k^{\times} \otimes k \rightarrow K_{1}\left(k[t],\left(t^{2}\right)\right) \rightarrow 0
$$

[Bloch and Esnault 2003, Proposition 2.1 and Corollary 2.5]. Then we have

$$
K_{2}\left(k[t],\left(t^{2}\right)\right)_{\mathbb{Q}}^{(2)} \simeq K_{3}\left(k[t]_{2},(t)\right)_{\mathbb{Q}}^{(2)} \quad \text { and } \quad K_{1}\left(k[t],\left(t^{2}\right)\right) \simeq K_{2}\left(k[t]_{2},(t)\right)
$$

(loc. cit.). Therefore the complex $T B(k) \rightarrow k^{\times} \otimes k$ (tensored with $\mathbb{Q}$ ), really computes the motivic cohomology of $k[t]_{2}$ relative to $(t)$. Moreover Bloch and Esnault define a dilogarithm map on $T B(k)$ :

Theorem 1.2.1 [Bloch and Esnault 2003, Corollary 2.5]. Let $\mathfrak{m}$ be the maximal ideal of $R$. There is a well-defined map $\rho: T B(k) \rightarrow \mathfrak{m}^{3} / \mathfrak{m}^{4}$ such that

$$
\rho(\langle a, b\rangle)=-a \cdot d b \quad \text { for }\langle a, b\rangle \in K_{2}\left(R,\left(t^{2}\right)\right) \text { with } a \in \mathfrak{m}^{2} \text { and } b \in R,
$$

and $\rho$ induces an isomorphism $K_{3}\left(k[t],\left(t^{2}\right)\right)^{(2)} \rightarrow \mathfrak{m}^{3} / \mathfrak{m}^{4}$ of abelian groups.

1.3. For $k$ a field of characteristic zero there is another natural complex, which is of more geometric origin and hence easier to relate to various definitions of categories of mixed Tate motives, that computes the weight two motivic cohomology groups of $k$.

Suppose $A$ is an artinian local ring with residue field $k$. The Bloch group $B_{2}(A)$ (denoted by $\mathfrak{p}(A)$ in [Suslin 1990]) is the free abelian group generated by the symbols $[x]$ such that $x(1-x) \in A^{\times}$, modulo the subgroup generated by elements of the form

$$
[x]-[y]+[y / x]-\left[\left(1-x^{-1}\right) /\left(1-y^{-1}\right)\right]+[(1-x) /(1-y)],
$$

for all $x, y \in A^{\times}$such that $(1-x)(1-y)(1-x / y) \in A^{\times}$. The map that sends $[x]$ to $x \wedge(1-x) \in \wedge^{2}{ }_{\mathbb{Z}} A^{\times}$induces the two term complex $\gamma_{A}$ (2) that sits in [1,2]:

$$
\delta_{A}: B_{2}(A) \rightarrow \bigwedge_{\mathbb{Z}}^{2} A^{\times} .
$$

The complex $\gamma_{k}(2)$ can be thought of as a more explicit version of $\Gamma_{k}(2)$. In fact, there is a natural map $\gamma_{k}(2)_{\mathbb{Q}} \rightarrow \Gamma_{k}(2)_{\mathbb{Q}}$, which is expected to be an isomorphism 
[Goncharov 1995, Conjecture 1.20], and there is an exact sequence [Suslin 1990]

$$
0 \rightarrow K_{3}(k)_{\mathbb{Q}}^{(2)} \rightarrow B_{2}(k)_{\mathbb{Q}} \rightarrow\left(\bigwedge^{2} k^{\times}\right)_{\mathbb{Q}} \rightarrow K_{2}(k)_{\mathbb{Q}} \rightarrow 0 .
$$

For $n \geq 2$, we are interested in the complex $\gamma_{k[\varepsilon]_{n}}(2)_{\mathbb{Q}}$, where $\delta_{k[\varepsilon]_{n}}$ will be denoted by $\delta_{n}$. We show that it has the expected cohomology:

Theorem 1.3.1. For $k$ a field of characteristic 0 , there is an exact sequence

$$
0 \rightarrow K_{3}\left(k[\varepsilon]_{n}\right)_{\mathbb{Q}}^{(2)} \longrightarrow B_{2}\left(k[\varepsilon]_{n}\right)_{\mathbb{Q}} \stackrel{\delta_{n}}{\longrightarrow}\left(\bigwedge^{2} k[\varepsilon]_{n}^{\times}\right)_{\mathbb{Q}} \longrightarrow K_{2}\left(k[\varepsilon]_{n}\right)_{\mathbb{Q}} \rightarrow 0 .
$$

For $n=2$ this theorem gives a "yes" answer to [Goncharov 2004, Problem 2.3].

While proving the previous theorem we construct an additive dilogarithm map on $B_{2}\left(k[\varepsilon]_{n}\right)$ :

Theorem 1.3.2. For every $n \geq 2$, there is a natural map

$$
\mathrm{Li}_{2, n}: B_{2}\left(k[\varepsilon]_{n}\right) \rightarrow k^{\oplus(n-1)}
$$

that, when composed with $K_{3}\left(k[\varepsilon]_{n},(\varepsilon)\right)^{(2)} \hookrightarrow B_{2}\left(k[\varepsilon]_{n}\right)$, induces an isomorphism $K_{3}\left(k[\varepsilon]_{n},(\varepsilon)\right)^{(2)} \simeq k^{\oplus(n-1)}$ of abelian groups.

The advantage of defining a dilogarithm map on $B_{2}\left(k[\varepsilon]_{n}\right)$ is that this group is closely related to the linear algebra-geometric complexes of mixed Tate motives. More precisely, $\mathrm{Li}_{2, n}$ immediately gives an analogue of the volume map for a pair of triangles over $k[\varepsilon]_{n}$, as in [Berllinson et al. 1990]: All one needs to do is to take the image of the pair of triangles in $B_{2}\left(k[\varepsilon]_{n}\right)$ under the map in [loc. cit., Proposition 3.7] and then apply $\mathrm{Li}_{2, n}$. In this context Theorems 1.3.1 and 1.3.2 imply that the class of a pair of triangles in $A_{2}\left(k\left[\varepsilon_{n}\right]\right) / A_{2}(k)$ (loc. cit.) is determined by its image in $\bigwedge^{2} k\left[\varepsilon_{n}\right]^{\times} / \bigwedge^{2} k^{\times}$and its image under $\operatorname{Li}_{2, n}$. This is a precise analogue of Sydler's theorem on Hilbert's 3rd problem that the scissors congruence class of a three-dimensional polyhedron is determined by its volume and its Dehn invariant [Goncharov 1999, Section 2.7]. We do not, however, pursue this application here.

1.4. In order to compare $\gamma_{k[\varepsilon]_{n}}(2)_{\mathbb{Q}}$ with the complex of Bloch and Esnault, we show that their argument extends to define a complex $T_{n} \mathbb{Q}(2)(k)$ by

$$
T_{n} B(k) \rightarrow k^{\times} \otimes\left(\varepsilon \cdot k[\varepsilon]_{n}\right)
$$

(for $n=2$ this is the complex in Section 1.2). Let $\gamma_{k[\varepsilon]_{n}}(2)_{\mathbb{Q}}=\gamma_{k}(2)_{\mathbb{Q}} \oplus \gamma_{k[\varepsilon]_{n}}(2)_{\mathbb{Q}}^{\circ}$, and note that the cohomology groups of $\gamma_{k[\varepsilon]_{n}}(2)_{\mathbb{Q}}^{\circ}$ and $T_{n} \mathbb{Q}(2)(k)$ coincide. We define a subcomplex $\gamma_{k[\varepsilon]_{n}}(2)_{\mathbb{Q}}^{\prime}$ of $\gamma_{k[\varepsilon]_{n}}(2)_{\mathbb{Q}}^{\circ}$ that has the same cohomology groups, and obtain a direct consequence of Theorems 1.2.1, 1.3.1, and 1.3.2:

Corollary 1.4.1. For $k$ an algebraically closed field of characteristic 0 , the complexes $T_{n} \mathbb{Q}(2)(k)$ and $\gamma_{k[\varepsilon]_{n}}(2)_{\mathbb{Q}}^{\prime}$ are isomorphic. 
1.5. The paper is organized as follows. In Section 2, we construct the additive dilogarithm, $\operatorname{Li}_{2, n}: B_{2}\left(k[\varepsilon]_{n}\right) \rightarrow k^{\oplus(n-1)}$. Two results in Section 2 are useful in studying $\mathrm{Li}_{2, n}$. On the one hand, $\mathrm{Li}_{2, n}$ is explicitly described in Proposition 2.2.3 and Definition 2.2.4. On the other hand, $\mathrm{Li}_{2, n}$ has a conceptual description: The image of an element in $B_{2}\left(k[\varepsilon]_{n}\right)$ under $\mathrm{Li}_{2, n}$ is obtained by lifting that element to an arbitrary element in $B_{2}\left(k[\varepsilon]_{2 n-1}\right)$ then taking its image in $\bigwedge^{2} k[\varepsilon]_{2 n-1}^{\times}$under the map in (1.3.1), and finally choosing certain algebraic combinations of its coordinates in $\bigwedge^{2} k[\varepsilon]_{2 n-1}^{\times}$as in Propositions 2.1.2, 2.2.1 and 2.2.2. It is this flexibility in the choice of the lifting that is used in the computations in Section 4.

In this paper, rather than working with K-theory we work with cyclic homology most of the time. This is possible since $K_{*}\left(k[\varepsilon]_{n}\right)=K_{*}\left(k[\varepsilon]_{n},(\varepsilon)\right) \oplus K_{*}(k)$ and by the theorem of Goodwillie [1986], $\mathrm{HC}_{*-1}\left(k[\varepsilon]_{n},(\varepsilon)\right) \simeq K_{*}\left(k[\varepsilon]_{n},(\varepsilon)\right)$, where $\mathrm{HC}$ denotes cyclic homology with respect to $\mathbb{Q}$. Note that since we are working with $\mathbb{Q}$-coefficients, $\mathrm{K}$-theory is nothing other than the primitive part of the rational homology of GL [Loday 1992, Corollary 11.2.12].

In Sections 3.1 through 3.6 we make Goodwillie's theorem explicit, following [Loday 1992], by giving the description of a map from $\mathrm{HC}_{2}\left(k[\varepsilon]_{n},(\varepsilon)\right)$ to $H_{3}\left(\mathrm{GL}\left(k[\varepsilon]_{n}\right), \mathbb{Q}\right)$. Then in Sections 3.7 and 3.8, Suslin and Guin's stability theorem and a construction of Bloch, Suslin and Goncharov is used to construct a map $H_{3}\left(\mathrm{GL}\left(k[\varepsilon]_{n}\right), \mathbb{Q}\right) \rightarrow \operatorname{ker}\left(\delta_{n}\right)$. More details about Section 3 are given in Section 3.1. This explicit description will be needed in Section 4.

In Section 4, we prove Theorem 1.3.2. This is done by first using the description of $\mathrm{HC}_{2}\left(k[\varepsilon]_{n},(\varepsilon)\right)$ given in [Cathelineau 1990/91] in Section 4.1.1, then constructing certain elements

$$
\alpha_{w} \in \mathrm{HC}_{2}\left(k[\varepsilon]_{n}, \varepsilon\right)^{(1)} \text { for } n+1 \leq w \leq 2 n-1,
$$

and chasing the images of these elements under the maps described in Sections 2 and 3. The proof also shows that $\left\{\alpha_{w}\right\}_{n+1 \leq w \leq 2 n-1}$ form a basis for $\mathrm{HC}_{2}\left(k[\varepsilon]_{n}, \varepsilon\right)^{(1)}$.

In Section 5, using [Suslin 1990], [Guin 1989], and Section 4, we show that the infinitesimal part of $\operatorname{ker}(\delta)$ is canonically isomorphic to $\mathrm{HC}_{2}\left(k[\varepsilon]_{n},(\varepsilon)\right)^{(1)}$. From this Theorem 1.3.1 follows.

In Section 6, we first define a subcomplex $\gamma_{k[\varepsilon]_{n}}(2)_{\mathbb{Q}}^{\prime}$ of $\gamma_{k[\varepsilon]_{n}}(2)_{\mathbb{Q}}$. Then we extend the construction of Bloch and Esnault to higher moduli and finally prove Corollary 1.4.1, which compares the two constructions.

Remarks. First, we mention the work of J. Park [2007], which gives an additive Chow theoretic description of the additive dilogarithm of Bloch and Esnault, and the work of K. Rülling [2007], which proves that the complex of additive Chow groups with modulus (not necessarily of 2) has the expected cohomology groups on the level of zero cycles. 
Second, there are many problems left unanswered in this note. The most important of these is the construction of additive polylogarithms for higher weights. We have made this construction, but we have yet to prove that the complex has the right cohomology groups. We will address in another paper the question of what happens in characteristic $p$, and we will also compare our construction to the work of Park and Rülling.

\section{Additive dilogarithm}

Notation 2.0.1. Let $k$ be a field of characteristic zero. An abelian group $A$ endowed with a group homomorphism $k^{\times} \rightarrow \operatorname{Aut}_{a b}(A)$ is said to be a $k^{\times}$-abelian group; we denote the action of $\lambda \in k^{\times}$on $a \in A$ by $\lambda \times a$. If $f: A \rightarrow k$ is an additive map that satisfies $f(\lambda \times a)=\lambda^{w} \cdot f(a)$ for all $\lambda \in k^{\times}$and $a \in A$, then we say that $f$ is of $k^{\times}$-weight $w$.

If $V$ is a $k$-module with a $k^{\times}$-action that is $k$-linear, that is, defined by a homomorphism $k^{\times} \rightarrow \operatorname{Aut}_{k \text {-mod }}(V)$, then we let

$$
V_{\langle w\rangle}:=\left\{v \in V \mid \lambda \times v=\lambda^{w} \cdot v \text { for all } \lambda \in k^{\times}\right\}
$$

be the subspace of elements of $V$ of weight $w$.

Define $k[\varepsilon]_{m}:=k[\varepsilon] /\left(\varepsilon^{m}\right), V_{m}:=k[\varepsilon]_{m}^{\times} \otimes_{\mathbb{Z}} \mathbb{Q}$ and $B_{2}\left(k[\varepsilon]_{m}\right)$ as in Section 1.3.

For an object $A$ defined over $k[\varepsilon]_{m}$, we denote by $A^{\circ}$ its infinitesimal part, for example,

$$
B_{2}\left(k[\varepsilon]_{m}\right)=B_{2}(k) \oplus B_{2}\left(k[\varepsilon]_{m}\right)^{\circ}, \quad k[\varepsilon]_{m}^{\circ}=\varepsilon \cdot k[\varepsilon]_{m}, \quad V_{m}^{\circ}=1+\varepsilon \cdot k[\varepsilon]_{m} .
$$

When the context requires it we write (say) $K_{*}\left(k[\varepsilon]_{m}\right)^{\circ}$ instead of $K_{*}\left(k[\varepsilon]_{m},(\varepsilon)\right)$. Finally, since in what follows the infinitesimal part $A^{\circ}$ of an object $A$ is canonically a direct summand of $A$, we never mention the natural maps $A^{\circ} \rightarrow A$ and $A \rightarrow A^{\circ}$, and take other liberties of this kind.

The exponential map gives an isomorphism $k[\varepsilon]_{m}^{\circ} \simeq V_{m}^{\circ}$, which endows $V_{m}^{\circ}$ with a $k$-space structure. For $\lambda \in k^{\times}$, the $k$-algebra map that sends $\varepsilon$ to $\lambda \cdot \varepsilon$ defines an action of $k^{\times}$on $k[\varepsilon]_{m}$ and $V_{m}^{\circ}$. Denote the weight $i$ subspace of $V_{m}^{\circ}$ under this action by $V_{m,\langle i\rangle}$, that is,

$$
V_{m,\langle i\rangle}=\left\{v \in V_{m}^{\circ} \mid \lambda \times v=\lambda^{i} \cdot v \text { for all } \lambda \in k^{\times}\right\}=\left\{\exp \left(a \cdot \varepsilon^{i}\right) \mid a \in k\right\} .
$$

Then $V_{m}^{\circ}=\bigoplus_{1 \leq i \leq m-1} V_{m,\langle i\rangle}$. To simplify the notation we also put $V_{m,\langle 0\rangle}:=k^{\times} \otimes \mathbb{Q}$.

Let $k[\varepsilon]_{m}^{\times \times} \subseteq k[\varepsilon]_{m}$ denote the set of exceptional units, that is, those $a \in k[\varepsilon]_{m}^{\times}$ such that $1-a \in k[\varepsilon]^{\times}$.

Let $\delta: \mathbb{Q}\left[k[\varepsilon]_{m}^{\times \times}\right] \rightarrow \bigwedge^{2} V_{m}$ be the map that sends $x \in k[\varepsilon]_{m}^{\times \times}$to $x \wedge(1-x)$. If we want to emphasize that we are working over $k[\varepsilon]_{m}$, we will use the notation $\delta_{m}$ 
instead of $\delta$. The map on $B_{2}\left(k[\varepsilon]_{m}\right)$ induced by $\delta_{m}$ is denoted by the same letter (see (1.3.1)).

2.1. Construction of $\mathbf{l i}_{2}$. In this section we collect the combinatorial arguments in the construction of the additive dilogarithm over $k[\varepsilon]_{n}$. The crucial step is the statement that $S_{k}(m, n)_{\langle w\rangle}$ is one dimensional in Proposition 2.1.2. This implies that if one thinks that the additive dilogarithm on $k[\varepsilon]_{n}$ should be constructed by first lifting to $k[\varepsilon]_{2 n-1}$ and then using $\delta$, then there is essentially one way to define it. That this is the right definition is justified in the next section.

Definition 2.1.1. Let $n, m \in \mathbb{N}$ such that $2 \leq n \leq m$. Let $\alpha_{m, n}: \mathbb{Q}\left[k[\varepsilon]_{m}^{\times \times}\right] \rightarrow \bigwedge^{2} V_{m}$ denote the map that sends $\gamma \in k[\varepsilon]_{m}^{\times \times}$to $\delta(\gamma)-\delta(\gamma \mid n)$, where $\gamma \mid n$ is the truncation of $\gamma$ to the sum of first $n$ powers of $\varepsilon$, that is, if $\gamma=a_{0}+a_{1} \cdot \varepsilon+\cdots+a_{m-1} \cdot \varepsilon^{m-1}$ then $\gamma \mid n=a_{0}+a_{1} \cdot \varepsilon+\cdots+a_{n-1} \cdot \varepsilon^{n-1}$.

Let $V(m, n)$ denote

$$
\bigoplus_{\substack{0<i \leq n-1 \\ n \leq j \leq m-1}}\left(V_{m,\langle i\rangle} \otimes V_{m,\langle j\rangle}\right) \subseteq \bigwedge^{2} V_{m},
$$

which we also consider as a quotient of $\bigwedge^{2} V_{m}$ via the direct sum decomposition

$$
\bigwedge^{2} V_{m}=\bigoplus_{0 \leq i<j<m}\left(V_{m,\langle i\rangle} \otimes V_{m,\langle j\rangle}\right) \oplus\left(\bigoplus_{0 \leq i<m} \bigwedge^{2} V_{m,\langle i\rangle}\right)
$$

Finally denote by $V_{k}(m, n)$ the quotient

$$
\bigoplus_{\substack{0<i \leq n-1 \\ n \leq j \leq m-1}}\left(V_{m,\langle i\rangle} \otimes_{k} V_{m,\langle j\rangle}\right)
$$

of $V(m, n)$, by $p(m, n): \bigwedge^{2} V_{m} \rightarrow V_{k}(m, n)$ the canonical projection, by $S_{k}(m, n)$ the $k^{\times}$-abelian group $V_{k}(m, n) / \operatorname{im}\left(p(m, n) \circ \alpha_{m, n}\right)$ and by $S_{k}(m, n)_{\langle i\rangle}$ the image of $V_{k}(m, n)_{\langle i\rangle}$ in $S_{k}(m, n)$. This notation is justified by noting that $S_{k}(m, n)$ has a natural $k$-module structure induced from that of $V_{k}(m, n)$ such that its weight $i$ subspace is equal to $S_{k}(m, n)_{\langle i\rangle}$ and $S_{k}(m, n)=\bigoplus_{0<i} S_{k}(m, n)_{\langle i\rangle}$.

For $0<i<j<m$, let $p_{i, j}: \bigwedge^{2} V_{m} \rightarrow V_{m,\langle i\rangle} \otimes V_{m,\langle j\rangle}$ denote the projection determined by the decomposition (2.1.1). Then $l_{i, j}: \bigwedge^{2} V_{m} \rightarrow k$ is defined by letting $(\log \otimes \log )\left(p_{i, j}(\alpha)\right)=l_{i, j}(\alpha) \cdot\left(\varepsilon^{i} \otimes \varepsilon^{j}\right)$ in $k[\varepsilon]_{m} \otimes_{k} k[\varepsilon]_{m}$ for any $\alpha \in \bigwedge^{2} V_{m}$.

Proposition 2.1.2. Let $n, m, w \in \mathbb{N}$ such that $2 \leq n<w \leq \min (2 n-1, m)$. Then $S_{k}(m, n)_{\langle w\rangle}$ is a one-dimensional $k$-module. The unique linear functional

$$
\mathrm{li}_{2,(m, n), w}: S_{k}(m, n)_{\langle w\rangle} \rightarrow k
$$


such that $\mathrm{li}_{2,(m, n), w}\left(\exp (\varepsilon) \otimes \exp \left(\varepsilon^{w-1}\right)\right)=1$ is given by

$$
\operatorname{li}_{2,(m, n), w}=\sum_{1 \leq j \leq w-n} j \cdot l_{j, w-j} .
$$

Proof. Let $\mathrm{li}_{2,(m, n), w}$ denote the map from $\bigwedge^{2} V_{m}$ to $k$ given by the formula

$$
\mathrm{li}_{2,(m, n), w}=\sum_{1 \leq j \leq w-n} j \cdot l_{j, w-j} .
$$

We would like to see that $\mathrm{li}_{2,(m, n), w} \circ \alpha_{m, n}=0$. Fix

$$
x:=s+s(1-s) a_{1} \varepsilon+\cdots+s(1-s) a_{m-1} \varepsilon^{m-1} \in k[\varepsilon]_{m}^{\times \times} .
$$

Let $A_{m}:=\{1, \ldots, m-1\}$ and let $\left(A_{m}\right)^{\times \alpha}$ denote the cartesian product of $A_{m}$ with itself $\alpha$-times. Define $\mathfrak{p}:\left(A_{m}\right)^{\times \alpha} \rightarrow k$ by $\mathfrak{p}\left(i_{1}, \ldots, i_{\alpha}\right):=a_{i_{1}} \cdot a_{i_{2}} \cdots a_{i_{\alpha}}$, and $\mathfrak{w}:\left(A_{m}\right)^{\times \alpha} \rightarrow \mathbb{N}$ by $\mathfrak{w}\left(i_{1}, \ldots, i_{\alpha}\right):=i_{1}+i_{2}+\cdots+i_{\alpha}$. Note that even though $\mathfrak{p}$ depends on $x$, we suppress it from the notation. In order to simplify the notation let $A(\alpha):=\left(A_{m}\right)^{\times \alpha}$ and $B(\alpha):=\left(A_{m}\right)^{\times \alpha} \backslash\left(A_{n}\right)^{\times \alpha}$.

If $1 \leq \alpha, \beta \leq w$, let

$$
C(\alpha, \beta):=\{(a, b) \mid a \in A(\alpha), b \in B(\beta), \mathfrak{w}(a)+\mathfrak{w}(b)=w\} .
$$

Let the permutation group $S_{\alpha+\beta}$ on $\alpha+\beta$ letters act on $A(\alpha) \times A(\beta)$ by permuting the coordinates. On $C(\alpha, \beta) \subseteq A(\alpha) \times A(\beta)$ consider the following equivalence relation. If $(a, b),(c, d) \in C(\alpha, \beta)$, then $(a, b)$ and $(c, d)$ are equivalent if there exists a permutation $\sigma \in S_{\alpha+\beta}$ such that $(a, b)^{\sigma}=(c, d)$. Denote the equivalence class of $(a, b)$ by $[(a, b)]$ and the set of all equivalence classes by $\mathscr{S}(\alpha, \beta)$. Let $\mathfrak{p}([a, b])=\mathfrak{p}(a) \cdot \mathfrak{p}(b)$.

Assume from now on that $\alpha+\beta \leq w$. Note that since $w \leq 2 n-1$, any element $(a, b) \in C(\alpha, \beta)$ has a unique coordinate that is greater than or equal to $n$. Denote this coordinate by $e(a, b)$. Denote by $(a, b)_{0}$ the element of $C(\alpha, \beta)$ obtained by interchanging the last coordinate of $(a, b)$ with the coordinate containing $e(a, b)$.

Then we define a map $\imath: C(\alpha, \beta) \rightarrow C(\beta, \alpha)$ as follows. Let $(a, b) \in C(\alpha, \beta)$. Then $\imath(a, b) \in C(\beta, \alpha)$ is the element $(a, b)_{0}$, where we think of both $C(\alpha, \beta)$ and $C(\beta, \alpha)$ as subsets of $A(\alpha) \times A(\beta) \simeq A(\alpha+\beta) \simeq A(\beta) \times A(\alpha)$. This passes to equivalence classes and gives a map $\mathscr{S}(\alpha, \beta) \rightarrow \mathscr{S}(\beta, \alpha)$ that we continue to denote by $l$. Note that $\iota^{2}=1$, and if $G \in \mathscr{S}(\alpha, \beta)$, then $\mathfrak{p}(l(G))=\mathfrak{p}(G)$, and

$$
\sum_{(a, b) \in G} \mathfrak{w}(a)=\sum_{(c, d) \in l(G)} \mathfrak{w}(c) .
$$

Letting $z=a_{1} \varepsilon+a_{2} \varepsilon^{2}+\cdots+a_{m-1} \varepsilon^{m-1}$, we have

$$
x=s(1+(1-s) z) \text { and } 1-x=(1-s)(1-s z) .
$$


Computing in $k[\varepsilon]_{m}$, this gives

$$
\log (x / s)=-\sum_{\ell=1}^{m-1} \frac{(s-1)^{\ell} z^{\ell}}{\ell} \quad \text { and } \quad \log ((1-x) /(1-s))=-\sum_{\ell=1}^{m-1} \frac{s^{\ell} z^{\ell}}{\ell} .
$$

Since $z^{\alpha}=\sum_{u \in A(\alpha)} \mathfrak{p}(u) \varepsilon^{\mathfrak{w}(u)}$, we have

$$
\begin{aligned}
\log (x / s) & =-\sum_{\ell=1}^{m-1} \frac{(s-1)^{\ell}}{\ell} \sum_{u \in A(\ell)} \mathfrak{p}(u) \varepsilon^{\mathfrak{w}(u)}, \\
\log ((1-x) /(1-s)) & =-\sum_{\ell=1}^{m-1} \frac{s^{\ell}}{\ell} \sum_{u \in A(\ell)} \mathfrak{p}(u) \varepsilon^{\mathfrak{w}(u)} .
\end{aligned}
$$

Then $\operatorname{li}_{2,(m, n), w}\left(\alpha_{m, n}(x)\right)$ is equal to

$$
\begin{aligned}
& \sum_{\substack{1 \leq \alpha \leq w \\
1 \leq \beta \leq w}} \sum_{\substack{a \in A(\alpha) \\
b \in B(\beta) \\
\mathfrak{w}(a)+\mathfrak{w}(b)=w}} \frac{\mathfrak{w}(a) \cdot \mathfrak{p}(a) \cdot \mathfrak{p}(b)}{\alpha \cdot \beta} \cdot\left((s-1)^{\alpha} \cdot s^{\beta}-s^{\alpha} \cdot(s-1)^{\beta}\right) \\
& =\sum_{\substack{1 \leq \alpha \leq w \\
1 \leq \beta \leq w}}\left((s-1)^{\alpha} \cdot s^{\beta}-s^{\alpha} \cdot(s-1)^{\beta}\right) \sum_{G \in \mathscr{S}(\alpha, \beta)}\left(\frac{\mathfrak{p}(G)}{\alpha \cdot \beta}\right) \sum_{(a, b) \in G} \mathfrak{w}(a) .
\end{aligned}
$$

On the other hand

$$
\begin{aligned}
\sum_{G \in \mathscr{S}(\alpha, \beta)}\left(\frac{\mathfrak{p}(G)}{\alpha \cdot \beta}\right) \sum_{(a, b) \in G} \mathfrak{w}(a) & =\sum_{G \in \mathscr{S}(\alpha, \beta)}\left(\frac{\mathfrak{p}(l(G))}{\alpha \cdot \beta}\right) \sum_{(c, d) \in l(G)} \mathfrak{w}(c) \\
& =\sum_{G \in \mathscr{S}(\beta, \alpha)}\left(\frac{\mathfrak{p}(G)}{\beta \cdot \alpha}\right) \sum_{(a, b) \in G} \mathfrak{w}(a) .
\end{aligned}
$$

Therefore $\operatorname{li}_{2,(m, n), w}\left(\alpha_{m, n}(x)\right)=0$, and we have a linear functional

$$
\operatorname{li}_{2,(m, n), w}: S_{k}(m, n)_{\langle w\rangle} \rightarrow k .
$$

By the definition of $\mathrm{li}_{2,(m, n), w}$ it is clear that $\mathrm{li}_{2,(m, n), w}\left(\exp (\varepsilon) \otimes \exp \left(\varepsilon^{w-1}\right)\right)=1$.

To finish the proof we only need to show that the space of linear functionals on $S_{k}(m, n)_{\langle w\rangle}$ is generated by $\mathrm{li}_{2,(m, n), w}$, or equivalently that if $l$ is a linear combination of $\left\{l_{2, w-2}, l_{3, w-3}, \ldots, l_{w-n, n}\right\}$ such that $l\left(\alpha_{m, n}(x)\right)=0$ for all $x \in k[\varepsilon]_{m}^{\times \times}$, then $l$ is zero. So let $l=\sum_{2 \leq i \leq w-n} c_{i} \cdot l_{i, w-i}$ satisfy $l\left(\alpha_{m, n}(x)\right)=0$ for all $x \in k[\varepsilon]_{m}^{\times \times}$. Assume that $l \neq 0$ and let $i_{0}$ be the smallest integer $i$ such that $c_{i} \neq 0$. For all $s \in k^{\times \times}$and $a_{1}, a_{i_{0}-1}, a_{w-i_{0}} \in k$, we have

$l\left(\alpha_{m, n}\left(s+s(1-s) \cdot a_{1} \cdot \varepsilon+s(1-s) \cdot a_{i_{0}-1} \cdot \varepsilon^{i_{0}-1}+s(1-s) \cdot a_{w-i_{0}} \cdot \varepsilon^{w-i_{0}}\right)\right)=0$. 
If we denote the left hand side of the above equation by $l\left(s, a_{1}, a_{i_{0}-1}, a_{w-i_{0}}\right)$, then

$$
\begin{aligned}
c_{i_{0}} \cdot \frac{1}{2}\left((s-1)^{2} s-s^{2}(s-1)\right) \cdot\left(a_{1} \cdot a_{i_{0}-1} \cdot a_{w-i_{0}}\right) & \\
& =l\left(s, a_{1}, a_{i_{0}-1}, a_{w-i_{0}}\right)-l\left(s, a_{1}, 0, a_{w-i_{0}}\right)=0 .
\end{aligned}
$$

Therefore $c_{i_{0}}=0$, contradicting the assumption.

2.2. Construction of $\mathbf{L i}$. Using the construction in the previous section, we show that $\mathrm{li}_{2,(2 n-1, n), w}$ descends to a function on $B_{2}\left(k[\varepsilon]_{n}\right)$, as defined in Section 1.3.

Proposition 2.2.1. For $n+1 \leq w \leq 2 n-1$, the map

$$
\operatorname{li}_{2,(2 n-1, n), w} \circ \delta: \mathbb{Q}\left[k[\varepsilon]_{2 n-1}^{\times \times}\right] \rightarrow k
$$

factors through the canonical projection $\mathbb{Q}\left[k[\varepsilon]_{2 n-1}^{\times \times}\right] \rightarrow \mathbb{Q}\left[k[\varepsilon]_{n}^{\times \times}\right]$.

We denote the induced map from $\mathbb{Q}\left[k[\varepsilon]_{n}^{\times \times}\right]$to $k$ by $\operatorname{Li}_{2, n, w}$.

Proof. This follows from the fact that $\mathrm{li}_{2,(2 n-1, n), w} \circ \alpha_{2 n-1, n}=0$ by the construction in Proposition 2.1.2.

Proposition 2.2.2. The map $\operatorname{Li}_{2, n, w}: \mathbb{Q}\left[k[\varepsilon]_{n}^{\times \times}\right] \rightarrow k$ factors through the canonical projection $\mathbb{Q}\left[k[\varepsilon]_{n}^{\times \times}\right] \rightarrow B_{2}\left(k[\varepsilon]_{n}\right)$.

We continue to denote the induced map by $\mathrm{Li}_{2, n, w}$.

Proof. We need to show that for $x, y \in k[\varepsilon]_{n}^{\times \times}$such that $x / y \in k[\varepsilon]_{n}^{\times \times}$,

$$
\operatorname{Li}_{2, n, w}\left([x]-[y]+[y / x]-\left[\left(1-x^{-1}\right) /\left(1-y^{-1}\right)\right]+[(1-x) /(1-y)]\right)=0 .
$$

If $\tilde{x}$ and $\tilde{y}$ are arbitrary liftings of $x$ and $y$ to $k[\varepsilon]_{2 n-1}^{\times \times}$, then Proposition 2.2.1 implies that the left side of the last equation is equal to

$$
\left(\mathrm{li}_{2,(2 n-1), w} \circ \delta\right)\left([\tilde{x}]-[\tilde{y}]+[\tilde{y} / \tilde{x}]-\left[\left(1-\tilde{x}^{-1}\right) /\left(1-\tilde{y}^{-1}\right)\right]+[(1-\tilde{x}) /(1-\tilde{y})]\right) .
$$

The proposition then follows from the fact that

$$
\begin{gathered}
\delta\left([\tilde{x}]-[\tilde{y}]+[\tilde{y} / \tilde{x}]-\left[\left(1-\tilde{x}^{-1}\right) /\left(1-\tilde{y}^{-1}\right)\right]+[(1-\tilde{x}) /(1-\tilde{y})]\right)=0 . \\
\text { If } \underline{c}=\left(c_{1}, \ldots, c_{r}\right) \in \mathbb{N}^{r} \text { and } x=s+s(1-s) a_{1} \varepsilon+\cdots+s(1-s) a_{n-1} \varepsilon^{n-1} \in k[\varepsilon]_{n}^{\times \times}
\end{gathered}
$$
then

$$
\mathfrak{p}(x ; \underline{c}):=a_{c_{1}} \cdot a_{c_{2}} \cdots a_{c_{r}} \text { and } \quad \mathfrak{w}(\underline{c}):=c_{1}+\cdots+c_{r} .
$$

Let $C(\alpha):=\{1,2, \ldots, n-1\}^{\times \alpha}$.

Proposition 2.2.3. For $n+1 \leq w \leq 2 n-1$, we have

$$
\operatorname{Li}_{2, n, w}([x])=\sum_{1 \leq \alpha, \beta \leq w} \frac{(s-1)^{\alpha} \cdot s^{\beta}-s^{\alpha} \cdot(s-1)^{\beta}}{\alpha \cdot \beta} \sum_{\substack{(a, b) \in C(\alpha) \times C(\beta) \\ \mathfrak{w}(a) \leq w-n \\ \mathfrak{w}(a, b)=w}} \mathfrak{w}(a) \cdot \mathfrak{p}(x ;(a, b)) .
$$


Proof. Direct computation.

Definition 2.2.4. Define the additive dilogarithm $\operatorname{Li}_{2, n}: B_{2}\left(k[\varepsilon]_{n}\right) \rightarrow k^{\oplus(n-1)}$ by

$$
\mathrm{Li}_{2, n}:=\bigoplus_{n+1 \leq w \leq 2 n-1} \mathrm{Li}_{2, n, w} .
$$

\section{The map from cyclic homology to the Bloch group}

3.1. This section is based on Goodwillie's theorem [1986] and the construction of Bloch [1977], Suslin [1990] and Goncharov [1995] of a map from the $K_{3}$ of a field to its Bloch group. Our main reference for cyclic homology and Goodwillie's theorem is [Loday 1992]. Here all cyclic homology groups are relative to $\mathbb{Q}$.

We will need the following to pass from cyclic homology to the rational homology of GL.

Theorem 3.1.1 [Goodwillie 1986; Loday 1992, Theorem 11.3.1]. Let A be a $\mathbb{Q}$-algebra and I a nilpotent ideal in A. Then there is a canonical isomorphism

$$
\mathrm{HC}_{n-1}(A, I) \simeq K_{n}(A, I)_{\mathbb{Q}} \text { for } n \geq 1 .
$$

Remark 3.1.2. This isomorphism is compatible with the $\lambda$-structures on both sides by [Cathelineau 1990/91, Theorem 1]. Hence, if $\operatorname{HC}_{*}(A, I)^{(i-1)}$ and $K_{*}(A, I)_{\mathbb{Q}}^{(i)}$ denote the $k^{i}$-eigenspace for the $k$-th Adams operator (for any $k$ ), then the above isomorphism induces an isomorphism $\mathrm{HC}_{*-1}(A, I)^{(i-1)} \simeq K_{*}(A, I)_{\mathbb{Q}}^{(i)}$ by [loc. cit., corollary in Section 1.3].

For a ring $A$, the Hurewicz map induces an isomorphism from $\oplus_{n>0} K_{n}(A)_{\mathbb{Q}}$ to the primitive part Prim $H_{*}(\mathrm{GL}(A), \mathbb{Q})$ of the homology of GL [Loday 1992, 11.2.12 Corollary]. The map in Theorem 3.1.1 is constructed as the composition of a map from cyclic homology to the primitive part of the homology of GL and then using the inverse of the Hurewicz map. Since we will only need the map from cyclic homology to the homology of GL, we next describe the steps in its construction, following [Loday 1992].

In Section 3.2, cyclic homology of $A$ is computed as the homology of the Connes complex. This section also describes the natural map from the Connes complex to the Chevalley-Eilenberg complex of the Lie algebra gl. This map induces an isomorphism from cyclic homology to the primitive homology of $\mathfrak{g l}$. In Section 3.3, homology of $\mathfrak{g l}$ is replaced with the sum of the homology of its nilpotent parts $\mathfrak{t}_{\sigma}(A, I)$. In Section 3.4, homology of $\mathfrak{t}_{\sigma}(A, I)$ is replaced with that of the completion of its universal enveloping algebra, and in Section 3.5, the latter is replaced with the homology of the group algebra of $T_{\sigma}(A, I)$, via Malčev theory. We reach the group homology of GL(A) in Section 3.6. 
In Section 3.7, this construction in combination with Suslin and Guin's stability theorem [Suslin 1984; Guin 1989] induces a map

$$
\mathrm{HC}_{n-1}(A, I) \rightarrow H_{n}\left(\mathrm{GL}_{n}(A), \mathbb{Q}\right)
$$

when $A$ is an artinian local algebra over $\mathbb{Q}$ and $I$ is a proper ideal of $A$. We will use this map for $n=3$.

Finally we use a slight variation of the construction of Suslin and Goncharov in Section 3.8 to get a map $H_{3}\left(\mathrm{GL}_{3}(A), \mathbb{Q}\right) \rightarrow \operatorname{ker}(\delta)$.

The details can be found in [Loday 1992, Section 11.3] and the references therein. The main result of this section is Proposition 3.8.9.

\subsection{Map from cyclic homology to Lie algebra homology.}

3.2.1. For any associative $\mathbb{Q}$-algebra $A$, the Connes complex $C_{*}^{\lambda}(A)$ is defined as follows. Let $\mathbb{Z} / n \mathbb{Z}$ act on $A^{\otimes n}$ by

$$
1 \times\left(a_{1} \otimes a_{2} \otimes \cdots \otimes a_{n}\right)=(-1)^{n-1} a_{2} \otimes a_{3} \otimes \cdots \otimes a_{n} \otimes a_{1},
$$

and let $C_{n-1}^{\lambda}(A)$ denote the coinvariants of $A^{\otimes n}$ under this action. Define

$$
\begin{aligned}
& b: C_{n}^{\lambda}(A) \rightarrow C_{n-1}^{\lambda}(A), \\
&\left(a_{0}, a_{1}, \ldots, a_{n}\right) \mapsto \sum_{0 \leq i \leq n-1}(-1)^{i}\left(a_{0}, \ldots, a_{i} \cdot a_{i+1}, \ldots, a_{n}\right) \\
&+(-1)^{n}\left(a_{n} \cdot a_{0}, a_{1}, \ldots, a_{n-1}\right) .
\end{aligned}
$$

Then $C_{*}^{\lambda}(A)$ is the complex

$$
\cdots \stackrel{b}{\longrightarrow} C_{n+1}^{\lambda}(A) \stackrel{b}{\longrightarrow} C_{n}^{\lambda}(A) \stackrel{b}{\longrightarrow} \cdots \longrightarrow C_{0}^{\lambda}(A) \longrightarrow 0,
$$

and $\mathrm{HC}_{*}(A)=H_{*}\left(C_{*}^{\lambda}(A)\right)$ [Loday 1992, Theorem 2.1.5]: The cyclic homology relative to $\mathbb{Q}$ can be computed as the homology of the Connes complex.

3.2.2. For $\mathfrak{g}$ a Lie algebra over $\mathbb{Q}$, the Chevalley-Eilenberg complex $C_{*}(\mathfrak{g}, \mathbb{Q})$ of $\mathfrak{g}$ with coefficients in $\mathbb{Q}$ is defined by

$$
\cdots \stackrel{d}{\longrightarrow} \bigwedge^{n} \mathfrak{g} \stackrel{d}{\longrightarrow} \bigwedge^{n-1} \mathfrak{g} \longrightarrow \cdots \stackrel{d}{\longrightarrow} \mathfrak{g} \stackrel{d}{\longrightarrow} \mathbb{Q} \longrightarrow 0,
$$

where $d: \bigwedge^{n} \mathfrak{g} \rightarrow \bigwedge^{n-1} \mathfrak{g}$ is given by

$d\left(a_{1} \wedge a_{2} \wedge \cdots \wedge a_{n}\right)=\sum_{1 \leq i<j \leq n}(-1)^{i+j-1}\left[a_{i}, a_{j}\right] \wedge a_{1} \wedge \cdots \wedge \hat{a}_{i} \wedge \cdots \wedge \hat{a}_{j} \wedge \cdots \wedge a_{n}$.

The Lie algebra homology $H_{*}(\mathfrak{g}, \mathbb{Q})$ of $\mathfrak{g}$ with coefficients in $\mathbb{Q}$ is the homology of the complex $C_{*}(\mathfrak{g}, \mathbb{Q})$. The diagonal map $\mathfrak{g} \rightarrow \mathfrak{g} \oplus \mathfrak{g}$ induces a map

$$
\Delta: C_{*}(\mathfrak{g}, \mathbb{Q}) \rightarrow C_{*}(\mathfrak{g}, \mathbb{Q}) \otimes C_{*}(\mathfrak{g}, \mathbb{Q}),
$$


which makes $\left(C_{*}(\mathfrak{g}, \mathbb{Q}), d\right)$ a DG-coalgebra. Let Prim $H_{*}(\mathfrak{g}, \mathbb{Q})$ denote the primitive elements in $H_{*}(\mathfrak{g}, \mathbb{Q})$, that is, those $\alpha$ such that $\Delta(\alpha)=1 \otimes \alpha+\alpha \otimes 1$.

Let $\mathfrak{g l}_{n}(A)$ denote the Lie algebra of $n \times n$ matrices, and let $\mathfrak{g l}(A)$ denote the direct $\operatorname{limit}_{\lim } \rightarrow \infty \mathfrak{g l}_{n}(A)$ with respect to the natural inclusions $\mathfrak{g l}_{n}(A) \subseteq \mathfrak{g l}_{m}(A)$ for $n \leq m$. Then $\mathfrak{g l}(\mathbb{Q})$ acts on $C_{*}(\mathfrak{g l}(A), \mathbb{Q})$ by

$$
\left[h, g_{1} \wedge \cdots \wedge g_{n}\right]:=\sum_{1 \leq i \leq n} g_{1} \wedge \cdots \wedge\left[h, g_{i}\right] \wedge \cdots \wedge g_{n}
$$

Let $C_{*}(\mathfrak{g l}(A), \mathbb{Q})_{\mathfrak{g l}(\mathbb{Q})}$ denote the complex of coinvariants with respect to this action, and let $H_{*}(\mathfrak{g l}(A), \mathbb{Q})_{\mathfrak{g l}(\mathbb{Q})}$ and Prim $H_{*}(\mathfrak{g l}(A), \mathbb{Q})_{\mathfrak{g l}(\mathbb{Q})}$ denote respectively the homology and the primitive part of the homology of the complex $C_{*}(\mathfrak{g l}(A), \mathbb{Q})_{\mathfrak{g l} l(\mathbb{Q})}$. Then the theorem of Loday, Quillen, and Tsygan says this:

Theorem 3.2.1 [Loday 1992, Theorem 10.2.4]. If $A$ is an algebra over $\mathbb{Q}$, then there is a natural isomorphism

$$
\mathrm{HC}_{*-1}(A) \simeq \operatorname{Prim} H_{*}(\mathfrak{g l}(A), \mathbb{Q})_{\mathfrak{g} l(\mathbb{Q})} \simeq \operatorname{Prim} H_{*}(\mathfrak{g} l(A), \mathbb{Q}) .
$$

Explicitly, the first isomorphism above is induced by the chain map that sends the class of $a_{1} \otimes a_{2} \otimes \cdots \otimes a_{n}$ in $C_{n-1}^{\lambda}(A)$ to the class of $a_{1} e_{12} \wedge a_{2} e_{23} \wedge \cdots \wedge a_{n} e_{n 1}$ in $C_{n}(\mathfrak{g l}(A), \mathbb{Q})_{\mathfrak{g l}(\mathbb{Q})}$. Here $e_{i j}$ denotes the matrix whose only nonzero entry is the one in the $i$-th row and the $j$-th column, which is 1 .

3.3. Volodin's construction in the Lie algebra case. Assume that $I$ is a nilpotent ideal of $A$, and let $\mathrm{HC}_{*}(A, I)$ denote the cyclic homology of $A$ relative to $I$, the homology of the complex $C_{*}^{\lambda}(A, I)$ that is the kernel of the natural surjection $C_{*}^{\lambda}(A) \rightarrow C_{*}^{\lambda}(A / I)$.

For any permutation $\sigma \in S_{n}$, let $\mathfrak{t}_{\sigma}(A, I)$ denote the Lie subalgebra of $\mathfrak{g l}(A)$ given by $\mathfrak{t}_{\sigma}(A, I):=\left\{\left(a_{i j}\right) \in \mathfrak{g l}(A): a_{i j} \in I\right.$ if $\left.\sigma(j) \leq \sigma(i)\right\}$. Let $x(A, I):=$ $\sum_{\sigma} C_{*}\left(\mathfrak{t}_{\sigma}(A, I), \mathbb{Q}\right)$ denote the sum of the subcomplexes

$$
C_{*}\left(\mathfrak{t}_{\sigma}(A, I), \mathbb{Q}\right) \subseteq C_{*}(\mathfrak{g l}(A), \mathbb{Q}),
$$

over all $n$ and $\sigma \in S_{n}$, and let $H_{*}(\mathfrak{g l}(A, I), \mathbb{Q})$ denote the homology of $x(A, I)$. Then the map in Theorem 3.2.1 induces an isomorphism

$$
\mathrm{HC}_{*-1}(A, I) \simeq \operatorname{Prim} H_{*}(\mathfrak{g l}(A, I), \mathbb{Q}) \simeq \sum_{\sigma} \operatorname{Prim} H_{*}\left(\mathfrak{t}_{\sigma}(A, I), \mathbb{Q}\right)
$$

[Loday 1992, Proposition 11.3.12].

3.4. From the Lie algebra to the universal enveloping algebra. For a Lie algebra $\mathfrak{g}$ over $\mathbb{Q}$, let $U(\mathfrak{g})$ denote its universal enveloping algebra and $\hat{U}(\mathfrak{g})$ its completion with respect to its augmentation ideal. We will next express the homology of $\mathfrak{g}$ in terms of the homology of $\mathcal{U}(\mathfrak{g})$. 
Let $B$ be an associative algebra over $\mathbb{Q}$ endowed with an augmentation map $\varepsilon: B \rightarrow \mathbb{Q}$. Let $C_{*}(B, \mathbb{Q})$ denote the complex

$$
\cdots \stackrel{b}{\longrightarrow} B^{\otimes n} \stackrel{b}{\longrightarrow} B^{\otimes(n-1)} \stackrel{b}{\longrightarrow} \cdots \stackrel{b}{\longrightarrow} \mathbb{Q} \longrightarrow 0,
$$

where $b: B^{\otimes n} \rightarrow B^{\otimes(n-1)}$ is the map that sends $b_{1} \otimes \cdots \otimes b_{n}$ to

$$
\begin{aligned}
\varepsilon\left(b_{1}\right) \cdot b_{2} \otimes \cdots \otimes b_{n}+\sum_{1 \leq i \leq n-1}(-1)^{i} b_{1} \otimes \cdots \otimes b_{i} \cdot b_{i+1} \otimes \cdots \otimes b_{n} \\
+(-1)^{n} \varepsilon\left(b_{n}\right) \cdot b_{1} \otimes \cdots \otimes b_{n-1} .
\end{aligned}
$$

Let $H_{*}(B, \mathbb{Q})$ denote the homology of this complex.

Then the natural maps

$$
H_{*}\left(\mathfrak{t}_{\sigma}(A, I), \mathbb{Q}\right) \simeq H_{*}\left(\mathcal{U}\left(\mathfrak{t}_{\sigma}(A, I)\right), \mathbb{Q}\right) \simeq H_{*}\left(\hat{\imath}\left(\mathfrak{t}_{\sigma}(A, I)\right), \mathbb{Q}\right)
$$

are isomorphisms [Loday 1992, Theorem 3.3.2]. Here the first map is induced by the chain map $\alpha_{\text {as }}$, where "as" stands for antisymmetrization, defined by

$$
\alpha_{\mathrm{as}}\left(t_{1} \wedge \cdots \wedge t_{n}\right)=\sum_{\tau \in S_{n}} \operatorname{sign}(\tau) \cdot t_{\tau(1)} \otimes \cdots \otimes t_{\tau(n)} .
$$

3.5. Malčev theory. For $\sigma \in S_{n}$, let $T_{\sigma}(A, I) \subseteq \mathrm{GL}_{n}(A)$ denote the group

$$
\left\{1+\left(a_{i j}\right) \in \mathrm{GL}_{n}(A) \mid a_{i j} \in I \text { if } \sigma(j) \leq \sigma(i)\right\} .
$$

For a discrete group $G$, denote by $U(G)$ its group $\operatorname{ring}$ over $\mathbb{Q}$, and by $\hat{U}(G)$ its completion with respect to the augmentation ideal.

Since $T_{\sigma}(A, I)$ is a unipotent group with Lie algebra $\mathfrak{t}_{\sigma}(A, I)$, the natural maps

$$
H_{*}\left(\hat{U}\left(\mathfrak{t}_{\sigma}(A, I), \mathbb{Q}\right)=H_{*}\left(\hat{U}\left(T_{\sigma}(A, I), \mathbb{Q}\right) \simeq H_{*}\left(U\left(T_{\sigma}(A, I)\right), \mathbb{Q}\right) .\right.\right.
$$

are isomorphisms [Loday 1992, Section 11.3.13].

Combining (3.3.1), (3.4.1) and (3.5.1) we get a map

$$
\mathrm{HC}_{*-1}(A, I) \rightarrow \sum_{\sigma} H_{*}\left(U\left(T_{\sigma}(A, I)\right), \mathbb{Q}\right) \rightarrow H_{*}(U(\mathrm{GL}(A)), \mathbb{Q}) .
$$

3.6. Group homology. Let $G$ be any (discrete) group and $C_{*}(G, \mathbb{Q})$ the complex

$$
\cdots \stackrel{d}{\longrightarrow} \mathbb{Q}\left[G^{n+1}\right] \stackrel{d}{\longrightarrow} \mathbb{Q}\left[G^{n}\right] \stackrel{d}{\longrightarrow} \cdots \stackrel{d}{\longrightarrow} \mathbb{Q}[G] \longrightarrow 0,
$$

where $C_{n}(G, \mathbb{Q})=\mathbb{Q}\left[G^{n+1}\right]$ and $d$ is the map that sends $\left(g_{0}, g_{1}, \ldots, g_{n}\right)$ to

$$
\sum_{0 \leq i \leq n}(-1)^{i}\left(g_{0}, \ldots, \hat{g}_{i}, \ldots, g_{n}\right) \text {. }
$$

Let $G$ act on this complex by multiplication on the left, that is, $g \times\left(g_{0}, \ldots, g_{n}\right):=$ $\left(g \cdot g_{0}, \ldots, g \cdot g_{n}\right)$, and let $H_{*}(G, \mathbb{Q}):=H_{*}\left(C_{*}(G, \mathbb{Q})_{G}\right)$ where the subscript $G$ denotes the space of coinvariants. 
The natural map $C_{*}(U(G), \mathbb{Q}) \rightarrow C_{*}(G, \mathbb{Q})$ that sends $g_{1} \otimes g_{2} \otimes \cdots \otimes g_{n}$ to

$$
\left(1, g_{1}, g_{1} \cdot g_{2}, \ldots, g_{1} \cdot g_{2} \cdots g_{n}\right)
$$

induces an isomorphism $H_{*}(U(G), \mathbb{Q}) \rightarrow H_{*}(G, \mathbb{Q})$ [Loday 1992, Appendix C.3]. Applying this to GL(A) and combining with (3.5.2) we obtain the map

$$
\mathrm{HC}_{*-1}(A, I) \rightarrow H_{*}(\mathrm{GL}(A), \mathbb{Q}) .
$$

3.7. Suslin's stability theorem. Suslin's stability theorem [1990] was generalized by Guin:

Theorem 3.7.1 [Guin 1989, Section 2]. For any $1 \leq n$ and any artinian local algebra $A$ over $\mathbb{Q}$, the map $H_{n}\left(\mathrm{GL}_{n}(A), \mathbb{Q}\right) \rightarrow H_{n}(\mathrm{GL}(A), \mathbb{Q})$ induced by the inclusion $\mathrm{GL}_{n} \hookrightarrow \mathrm{GL}$ is an isomorphism.

Therefore if $A$ is an artinian local algebra over $\mathbb{Q}$ and $I$ is a proper ideal, we have a map $\rho_{1}: \mathrm{HC}_{n-1}(A, I) \rightarrow H_{n}\left(\mathrm{GL}_{n}(A), \mathbb{Q}\right)$.

3.8. Bloch-Suslin map. Let $A$ be an artinian local algebra over $\mathbb{Q}$ with residue field $k$. We now describe the Bloch-Suslin map [Goncharov 1995, Section 2.6]

$$
\rho_{2}: H_{3}\left(\mathrm{GL}_{3}(A), \mathbb{Q}\right) \rightarrow \operatorname{ker}\left(\delta_{A}\right),
$$

where $\delta_{A}: B_{2}(A)_{\mathbb{Q}} \rightarrow \bigwedge^{2} A_{\mathbb{Q}}^{\times}$is the differential in the Bloch complex.

Definition 3.8.1. Let $V$ be a finite free module over $A$, and denote $\tilde{C}_{m}(V)$ by the $\mathbb{Q}$ vector space with basis consisting of $m$-tuples $\left(x_{0}, \ldots, x_{m-1}\right)$ of elements of $V$ that are in general position, that is, for any $I \subseteq\{0,1, \ldots, m-1\}$ with $|I| \leq \operatorname{rank}(V)$, the set $\left\{x_{i} \mid i \in I\right\}$ can be extended to a basis of $V$. Let $C_{m}(V)$ denote the coinvariants of this space under the natural action of $\operatorname{GL}(V)$. Finally, let $\tilde{C}_{m}(p):=\tilde{C}_{m}\left(A^{\oplus p}\right)$ and $C_{m}(p):=C_{m}\left(A^{\oplus p}\right)$.

Remark 3.8.2. Let $\tilde{C}_{m}(\mathbb{P}(V))$ denote the $\mathbb{Q}$-space with basis $\left(v_{0}, \ldots, v_{m-1}\right)$ of $m$-tuples of points in $\mathbb{P}(V)$ that are in general position, and define

$d: \tilde{C}_{m+1}(\mathbb{P}(V)) \rightarrow \tilde{C}_{m}(\mathbb{P}(V)), \quad\left(v_{0}, \ldots, v_{m}\right) \mapsto \sum_{0 \leq i \leq m}(-1)^{i}\left(v_{0}, \ldots, \hat{v}_{i}, \ldots, v_{m}\right)$.

Let $C_{m}(\mathbb{P}(V))$ denote the space of coinvariants of $\tilde{C}_{m}(\mathbb{P}(V))$ under the natural action of $\operatorname{GL}(V)$. Then by identifying $[x]$ with $(0, x, 1, \infty) \in C_{4}\left(\mathbb{P}\left(A^{\oplus 2}\right)\right)$ and by comparing the dilogarithm relation in the definition of $B_{2}(A)$ to $d(0, x, y, 1, \infty)$ in $C_{4}\left(\mathbb{P}\left(A^{\oplus 2}\right)\right)$, we see that

$$
B_{2}(A)_{\mathbb{Q}}=C_{4}\left(\mathbb{P}\left(A^{\oplus 2}\right)\right) / d\left(C_{5}\left(\mathbb{P}\left(A^{\oplus 2}\right)\right)\right) .
$$

For $\left(x_{1}, \ldots, x_{4}\right)$ a quadruple of points in $\mathbb{P}_{A}^{1}$, we denote the corresponding element in $B_{2}(A)_{\mathbb{Q}}$ by $\left[x_{1}, \ldots, x_{4}\right]$. 
Since $A$ is a local ring, a subset of $V$ is in general position if its reduction modulo the maximal ideal is in general position in the $k$-space $V \otimes_{A} k$.

Define two maps $d, d^{\prime}: \tilde{C}_{m+1}(p) \rightarrow \tilde{C}_{m}(p)$ by

$$
\begin{aligned}
& d\left(x_{0}, x_{1}, \ldots, x_{m}\right)=\sum_{0 \leq i \leq m}(-1)^{i}\left(x_{0}, \ldots, \hat{x}_{i}, \ldots, x_{m}\right), \\
& d^{\prime}\left(x_{0}, x_{1}, \ldots, x_{m}\right)=\sum_{1 \leq i \leq m}(-1)^{i}\left(x_{0}, \ldots, \hat{x}_{i}, \ldots, x_{m}\right) .
\end{aligned}
$$

Let $\varepsilon: \tilde{C}_{1}(p) \rightarrow \mathbb{Q}$ be the map that sends each term to the sum of its coefficients.

Lemma 3.8.3. The following complexes are acyclic.

$$
\begin{aligned}
& \cdots \stackrel{d}{\longrightarrow} \tilde{C}_{2}(p) \stackrel{d}{\longrightarrow} \tilde{C}_{1}(p) \stackrel{\varepsilon}{\longrightarrow} \mathbb{Q} \longrightarrow 0, \\
& \cdots \stackrel{d^{\prime}}{\longrightarrow} \tilde{C}_{2}(p) \stackrel{d^{\prime}}{\longrightarrow} \tilde{C}_{1}(p) \longrightarrow 0 .
\end{aligned}
$$

Proof. Let $\sum_{j \in J} a_{j} \cdot\left(x_{0}(j), \ldots, x_{m-1}(j)\right)$ be an $m$-cycle in the first or the second complex. Since the reductions modulo the maximal ideal $\left\{\bar{x}_{0}(j), \ldots, \bar{x}_{m-1}(j)\right\}$ are in general position in $k^{\oplus p}$ and $k$ is an infinite field, we can choose $\alpha \in A$ such that all $\left\{\bar{x}_{0}(j), \ldots, \bar{x}_{m-1}(j), \bar{\alpha}\right\}$ are in general position. Note that if $W_{i}$ for $1 \leq i \leq r$ are proper subspaces of a vector space $W$ over an infinite field, then $\bigcup_{1 \leq i \leq r} W_{i} \neq W$. If $m \geq 2$ and $d \sum_{j \in J} a_{j} \cdot\left(x_{0}(j), \ldots, x_{m-1}(j)\right)=0$, or if $m=1$ and $\sum_{j \in J} a_{j}=0$, we have

$$
(-1)^{m} d\left(\sum_{j \in J} a_{j} \cdot\left(x_{0}(j), \ldots, x_{m-1}(j), \alpha\right)\right)=\sum_{j \in J} a_{j} \cdot\left(x_{0}(j), \ldots, x_{m-1}(j)\right) .
$$

Similarly, if $m \geq 2$ and $d^{\prime} \sum_{j \in J} a_{j} \cdot\left(x_{0}(j), \ldots, x_{m-1}(j)\right)=0$, or if $m=1$, we have

$$
(-1)^{m} d^{\prime}\left(\sum_{j \in J} a_{j} \cdot\left(x_{0}(j), \ldots, x_{m-1}(j), \alpha\right)\right)=\sum_{j \in J} a_{j} \cdot\left(x_{0}(j), \ldots, x_{m-1}(j)\right) .
$$

Define maps $\lambda: \tilde{C}_{m}(p) \rightarrow \tilde{C}_{m}(p)$ by

$$
\lambda\left(x_{0}, \ldots, x_{m-1}\right)=\sum_{0 \leq i \leq m-1} \operatorname{sign}\left(\sigma(m)^{i}\right)\left(x_{\sigma(m)^{i}(0)}, \ldots, x_{\sigma(m)^{i}(m-1)}\right),
$$

where $\sigma(m):=\left(\begin{array}{llll}0 & 1 & \cdots & m-1\end{array}\right)$ is the standard $m$-cyclic permutation.

Then $\lambda \circ d=d^{\prime} \circ \lambda$, and we have a double complex

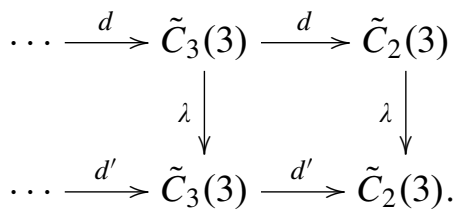


Definition 3.8.4. Let $\tilde{D}$ be the complex associated to the double complex above. That is, $\tilde{D}_{0}=\tilde{C}_{2}(3)$ and $\tilde{D}_{i}=\tilde{C}_{i+2}(3) \oplus \tilde{C}_{i+1}(3)$ and the maps are given by $(x, y) \rightarrow\left(d^{\prime}(x)+\lambda(y),-d(y)\right)$.

Let $\varepsilon: \tilde{D}_{0} \rightarrow \mathbb{Q}$ be the map that sends each term to the sum of its coefficients. Then by Lemma 3.8.3 the complex $\cdots \longrightarrow \tilde{D}_{1} \longrightarrow \tilde{D}_{0} \stackrel{\varepsilon}{\longrightarrow} \mathbb{Q} \longrightarrow 0$ is acyclic.

If we endow $\tilde{D}$ with its natural $\mathrm{GL}_{3}(A)$ action and $\mathbb{Q}$ with the trivial action, then the complex above is an acyclic complex of $\mathrm{GL}_{3}(A)$-modules. Therefore we get a canonical map

$$
H_{3}\left(\mathrm{GL}_{3}(A), \mathbb{Q}\right) \rightarrow H_{3}(D),
$$

where $D:=\tilde{D}_{\mathrm{GL}_{3}(A)}$ is the complex of coinvariants of $\tilde{D}$.

Next we define a map from $H_{3}(D)$ to $B_{2}(A)_{\mathbb{Q}}$. This will be a slight modification of Goncharov's map [1995, Section 2.6].

From the double complex above, we are interested in the part

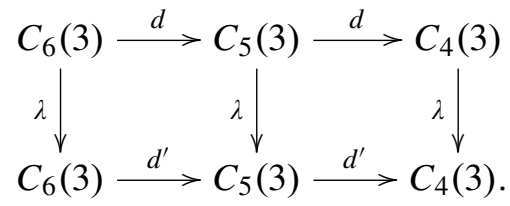

We define a map $\phi$ from this double complex to the double complex

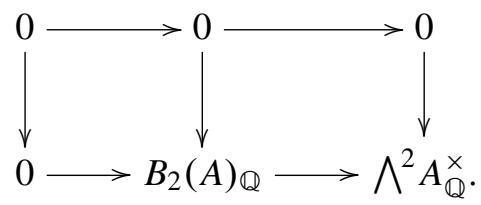

In $\phi$, the only nontrivial map

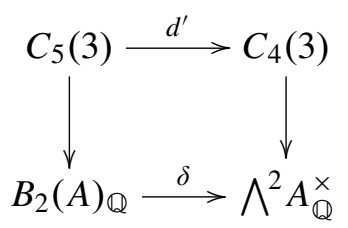

is a composition of the following two maps:

The first map is

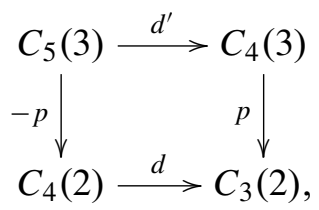

where $p: C_{m+1}(3) \rightarrow C_{m}(2)$ sends $\left(v_{0}, v_{1}, \ldots, v_{m-1}\right)$ to $\left(\tilde{v}_{1}, \ldots, \tilde{v}_{m-1}\right)$. Here $\tilde{v}_{i}$ denotes the image of $v_{i}$ in $A^{\oplus 3} /\left\langle v_{0}\right\rangle$, and the term $\left(\tilde{v}_{1}, \ldots, \tilde{v}_{m-1}\right)$ is identified with an element of $C_{m}(2)$ by choosing any isomorphism between $A^{\oplus 3} /\left\langle v_{0}\right\rangle$ and $A^{\oplus 2}$. 
The second map is

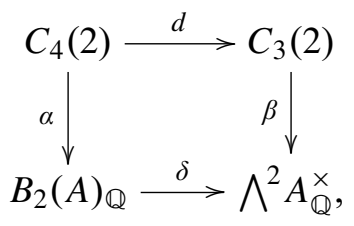

where $\alpha$ is the map that sends $\left(v_{0}, v_{1}, v_{2}, v_{3}\right)$ to $\left[\underline{v}_{0}, \underline{v}_{1}, \underline{v}_{2}, \underline{v}_{3}\right]$. Here $\underline{v}_{i}$ denotes the image of $v_{i}$ in $\mathbb{P}\left(A^{\oplus 2}\right)$, and $\left[\underline{v}_{0}, \underline{v}_{1}, \underline{v}_{2}, \underline{v}_{3}\right]$ denotes the image of $\left(\underline{v}_{0}, \underline{v}_{1}, \underline{v}_{2}, \underline{v}_{3}\right)$ under the map $C_{4}\left(\mathbb{P}\left(A^{\oplus 2}\right)\right) \rightarrow B_{2}(A)_{\mathbb{Q}}$, as in Remark 3.8.2. And $\beta$ is the map that sends $\left(v_{0}, v_{1}, v_{2}\right)$ to

$$
\left(\frac{v_{0} \wedge v_{1}}{v_{1} \wedge v_{2}}\right) \wedge\left(\frac{v_{0} \wedge v_{2}}{v_{1} \wedge v_{2}}\right)
$$

The next three lemmas imply that the maps defined so far can be extended to a map $\phi$ of the double complexes.

Lemma 3.8.5. The map $C_{6}(3) \stackrel{d^{\prime}}{\longrightarrow} C_{5}(3) \stackrel{-p}{\longrightarrow} C_{4}(2) \stackrel{\alpha}{\longrightarrow} B_{2}(A)_{\mathbb{Q}}$ is zero.

Proof. This follows from that $-p \circ d^{\prime}\left(v_{0}, v_{1}, v_{2}, v_{3}, v_{4}, v_{5}\right)=d\left(v_{1}, v_{2}, v_{3}, v_{4}, v_{5}\right)$, and that this maps to zero in $B_{2}(A)_{\mathbb{Q}}$, by Remark 3.8.2.

Lemma 3.8.6. The map $C_{5}(3) \stackrel{\lambda}{\longrightarrow} C_{5}(3) \stackrel{-p}{\longrightarrow} C_{4}(2) \stackrel{\alpha}{\longrightarrow} B_{2}(A)_{\mathbb{Q}}$ is zero.

Proof. (See [Goncharov 1995, Lemma 2.18].) Let $\left(v_{0}, \ldots, c_{4}\right) \in C_{5}(3)$. Then there is a conic $Q$ passing through the images of the five points $v_{0}, v_{1}, v_{2}, v_{3}, v_{4}$ in the projective plane. Choosing any isomorphism, we identify $Q$ with $\mathbb{P}_{A}^{1}$. Let the images of $v_{i}$ be $x_{i} \in \mathbb{P}_{A}^{1}$ under this isomorphism. The composition of the maps in the statement of the lemma then maps $\left(v_{0}, \ldots, v_{4}\right)$ in $C_{5}(3)$ to

$$
-\sum_{0 \leq i \leq 4}\left[x_{i}, x_{i+1}, \ldots, x_{i+3}\right]
$$

in $B_{2}(A)_{\mathbb{Q}}$, where the indices are modulo 5 .

Claim 3.8.7. In $B_{2}(A)_{\mathbb{Q}}$ we have

$$
\left[x_{1}, x_{2}, x_{3}, x_{4}\right]=\operatorname{sign}(\sigma) \cdot\left[x_{\sigma(1)}, x_{\sigma(2)}, x_{\sigma(3)}, x_{\sigma(4)}\right] \quad \text { for any } \sigma \in S_{4} .
$$

Proof of the claim. Note that since we are working with $\mathbb{Q}$-modules we have $[0, x, 1, \infty]=-[0, x /(x-1), 1, \infty]$ by [Suslin 1990, Lemmas 1.2 and 1.5]. Hence

$$
[0, x, 1, \infty]=-[x, 0,1, \infty] \text { and }[0, x, 1, \infty]=-[0,1 / x, 1, \infty]
$$

[loc. cit., Lemma 1.2]. Hence $[0, x, 1, \infty]=-[0,1, x, \infty]$, and using again that $[0, x, 1, \infty]=-[0, x /(x-1), 1, \infty]$, we have $[0, x, 1, \infty]=-[0, x, \infty, 1]$.

Therefore the formula in the statement holds for the transpositions (1 2), (2 3), and (34). Since these generate $S_{4}$, the statement follows. 
Finally by the claim,

$$
\sum_{0 \leq i \leq 4}\left[x_{i}, x_{i+1}, \ldots, x_{i+3}\right]=\sum_{0 \leq i \leq 4}(-1)^{i}\left[x_{0}, \ldots, \hat{x}_{i}, \ldots, x_{4}\right]
$$

and the right side is zero in $B_{2}(A)_{\mathbb{Q}}$ by Remark 3.8.2.

Lemma 3.8.8. The map $C_{4}(3) \stackrel{\lambda}{\longrightarrow} C_{4}(3) \stackrel{p}{\longrightarrow} C_{3}(2) \stackrel{\beta}{\longrightarrow} \bigwedge^{2} A_{\mathbb{Q}}^{\times}$is zero.

Proof. First note that $\beta$ sends $\left(v_{0}, v_{1}, v_{2}\right)$ to

$$
\left(\frac{v_{0} \wedge v_{1}}{v_{1} \wedge v_{2}}\right) \wedge\left(\frac{v_{0} \wedge v_{2}}{v_{1} \wedge v_{2}}\right)=\left(\frac{\ell\left(v_{0} \wedge v_{1}\right)}{\ell\left(v_{1} \wedge v_{2}\right)}\right) \wedge\left(\frac{\ell\left(v_{0} \wedge v_{2}\right)}{\ell\left(v_{1} \wedge v_{2}\right)}\right),
$$

where $\ell: \operatorname{det}_{A}\left(A^{\oplus 2}\right) \rightarrow A$ is any surjective $A$-linear map. Therefore since we are looking at configurations in general position, the composition $\beta \circ p$ maps $\left(y_{0}, y_{1}, y_{2}, y_{3}\right) \in C_{4}(3)$ to

$$
\left(\frac{y_{0} \wedge y_{1} \wedge y_{2}}{y_{0} \wedge y_{2} \wedge y_{3}}\right) \wedge\left(\frac{y_{0} \wedge y_{1} \wedge y_{3}}{y_{0} \wedge y_{2} \wedge y_{3}}\right)
$$

This implies the statement by direct computation.

Therefore $\phi$ is a map of double complexes that induces a map $H_{3}(D) \rightarrow \operatorname{ker}(\delta)$ of the homology of the associated complexes. Combining this with the map

$$
H_{3}\left(\mathrm{GL}_{3}(A), \mathbb{Q}\right) \rightarrow H_{3}(D)
$$

in (3.8.1), we obtain a map $\rho_{2}: H_{3}\left(\mathrm{GL}_{3}(A), \mathbb{Q}\right) \rightarrow \operatorname{ker}(\delta)$.

Therefore applying Sections $3.1-3.7$ to $(A, I)=\left(k[\varepsilon]_{n},(\varepsilon)\right)$ proves this:

Proposition 3.8.9. The composition $T:=\rho_{2} \circ \rho_{1}$ defines a natural map

$$
T: \mathrm{HC}_{2}\left(k[\varepsilon]_{n},(\varepsilon)\right)^{(1)} \hookrightarrow \mathrm{HC}_{2}\left(k[\varepsilon]_{n},(\varepsilon)\right) \rightarrow B_{2}\left(k[\varepsilon]_{n}\right)_{\mathbb{Q}},
$$

whose image lies in $\operatorname{ker}\left(\delta_{n}\right)$.

\section{Nonvanishing of $\mathrm{Li}_{2, n}$ on $\mathrm{HC}_{2}\left(k[\varepsilon]_{n},(\varepsilon)\right)^{(1)}$}

4.1. This section shows that $\mathrm{Li}_{2, n}$ is the correct map, as we show that it does not vanish on $\mathrm{HC}_{2}\left(k[\varepsilon]_{n},(\varepsilon)\right)^{(1)}$. First we describe $\mathrm{HC}_{2}\left(k[\varepsilon]_{n},(\varepsilon)\right)^{(1)}$ and define some elements $\alpha_{w}$ in it on which we will evaluate the additive dilogarithm.

4.1.1. Note that $\operatorname{HC}_{*}\left(k[\varepsilon]_{n},(\varepsilon)\right)$ is a $k^{\times}$-abelian group, where $\lambda \in k^{\times}$acts as the map induced by the $k$-algebra automorphism of $k[\varepsilon]_{n}$ that sends $\varepsilon$ to $\lambda \cdot \varepsilon$. This action is compatible with the decomposition (Remark 3.1.2) of

$$
\mathrm{HC}_{2}\left(k[\varepsilon]_{n},(\varepsilon)\right)=\mathrm{HC}_{2}\left(k[\varepsilon]_{n},(\varepsilon)\right)^{(1)} \oplus \mathrm{HC}_{2}\left(k[\varepsilon]_{n},(\varepsilon)\right)^{(2)}
$$


[Cathelineau 1990/91, pages 593-594];

$$
\mathrm{HC}_{2}\left(k[\varepsilon]_{n},(\varepsilon)\right)^{(1)}=\bigoplus_{n+1 \leq w \leq 2 n-1} \mathrm{HC}_{2}\left(k[\varepsilon]_{n},(\varepsilon)\right)_{\langle w\rangle}^{(1)},
$$

where each summand is isomorphic to $k$ (loc. cit.); and

$$
\mathrm{HC}_{2}\left(k[\varepsilon]_{n},(\varepsilon)\right)^{(2)}=\bigoplus_{1 \leq w \leq n-1} \mathrm{HC}_{2}\left(k[\varepsilon]_{n},(\varepsilon)\right)_{\langle w\rangle}^{(2)},
$$

where each summand is isomorphic to $\Omega_{k / \mathbb{Q}}^{2}$ (loc. cit.).

4.1.2. $\chi(n)=0$ if $n$ is even and $\chi(n)=1$ if $n$ is odd. For $n+1 \leq w \leq 2 n-1$, let

$$
\alpha_{w}:=\sum_{0 \leq j<(2 n-1-w) / 2}\left(\varepsilon^{n-1-j}, \varepsilon^{w-n+j}, \varepsilon\right)+\frac{1}{2} \cdot \chi(w) \cdot\left(\varepsilon^{(w-1) / 2}, \varepsilon^{(w-1) / 2}, \varepsilon\right)
$$

in $C_{2}^{\lambda}\left(k[\varepsilon]_{n}\right)$. Since $\alpha_{w}$ is a cycle, as can be checked by direct computation, with $k^{\times}$-weight $w$, it defines an element $\alpha_{w} \in \mathrm{HC}_{2}\left(k[\varepsilon]_{n},(\varepsilon)\right)_{\langle w\rangle}^{(1)}$ by Section 4.1.1.

4.2. Computation of $\mathbf{L i}_{2, n}$ on $\mathbf{H C}_{2}$. In this section, we compute $\operatorname{Li}_{2, n}\left(T\left(\alpha_{w}\right)\right)$ (which is the same as $\operatorname{Li}_{2, n, w}\left(T\left(\alpha_{w}\right)\right)$ ). This we will do in several steps.

4.2.1. From $\mathfrak{g l}_{3}\left(k[\varepsilon]_{n}\right)$ to $\mathfrak{g l}_{2}\left(k[\varepsilon]_{n}\right)$. Consider the 2 -chain $\left(\varepsilon^{a}, \varepsilon^{b}, \varepsilon\right) \in C_{2}^{\lambda}\left(k[\varepsilon]_{n}\right)$ in the Connes complex, where $a+b \geq n$. By the map in Section 3.2, at the chain complex level, $\left(\varepsilon^{a}, \varepsilon^{b}, \varepsilon\right)$ goes to $\beta_{a, b}:=\varepsilon^{a} e_{12} \wedge \varepsilon^{b} e_{23} \wedge \varepsilon e_{31} \in C_{3}\left(\mathfrak{g l}_{3}\left(k[\varepsilon]_{n}\right)\right)_{\mathfrak{g l}}(\mathbb{Q})$. Therefore we need to compute the image of

$$
\beta_{w}:=\sum_{0 \leq j<(2 n-1-w) / 2} \beta_{n-1-j, w-n+j}+\frac{1}{2} \chi(w) \beta_{(w-1) / 2,(w-1) / 2}
$$

in $k$. Let $\gamma_{a, b}:=\varepsilon^{a} e_{12} \wedge \varepsilon^{b} e_{21} \wedge \varepsilon e_{11}$, and

$$
\gamma_{w}:=\sum_{0 \leq j<(2 n-1-w) / 2} \gamma_{n-1-j, w-n+j}+\frac{1}{2} \chi(w) \gamma_{(w-1) / 2,(w-1) / 2} .
$$

We defined $T$ as the composition

$$
\begin{aligned}
\mathrm{HC}_{2}\left(k[\varepsilon]_{n},(\varepsilon)\right)^{(1)} & \rightarrow \operatorname{Prim} H_{3}\left(\mathfrak{g l}\left(k[\varepsilon]_{n}\right), \mathbb{Q}\right)_{\mathfrak{g l}(\mathbb{Q})} \simeq \operatorname{Prim} H_{3}\left(\mathfrak{g l}\left(k[\varepsilon]_{n}\right), \mathbb{Q}\right) \\
& \rightarrow H_{3}\left(\mathfrak{g l}\left(k[\varepsilon]_{n}\right), \mathbb{Q}\right) \rightarrow H_{3}\left(\mathrm{GL}\left(k[\varepsilon]_{n}\right), \mathbb{Q}\right) \rightarrow \operatorname{ker}(\delta) .
\end{aligned}
$$

Let $T^{\prime}: \operatorname{Prim} H_{3}\left(\mathfrak{g l}\left(k[\varepsilon]_{n}\right), \mathbb{Q}\right)_{\mathfrak{g l}(\mathbb{Q})} \rightarrow \operatorname{ker}(\delta)$ and $T^{\prime \prime}: H_{3}\left(\mathfrak{g l}\left(k[\varepsilon]_{n}\right), \mathbb{Q}\right) \rightarrow \operatorname{ker}(\delta)$ be the obvious compositions.

The following lemma enables us to work in the homology of $\mathfrak{g l}_{2}\left(k[\varepsilon]_{n}\right)$ rather than that of $\mathfrak{g l}_{3}\left(k[\varepsilon]_{n}\right)$.

Lemma 4.2.1. We have $\left(\operatorname{Li}_{2, n, w} \circ T^{\prime}\right)\left(\beta_{w}\right)=\left(\operatorname{Li}_{2, n, w} \circ T^{\prime}\right)\left(\gamma_{w}\right)$. 
Proof. First note that

$$
\begin{aligned}
d\left(e_{13} \wedge \varepsilon^{a} e_{12} \wedge \varepsilon^{b} e_{21} \wedge \varepsilon e_{31}\right)=- & \beta_{a, b}+\gamma_{a, b} \\
& -\varepsilon^{a} e_{12} \wedge \varepsilon^{b} e_{21} \wedge \varepsilon e_{33}-e_{13} \wedge \varepsilon^{a+1} e_{32} \wedge \varepsilon^{b} e_{21},
\end{aligned}
$$

that $\varepsilon^{a} e_{12} \wedge \varepsilon^{b} e_{21} \wedge \varepsilon e_{33}$ is a cycle; and that $e_{13} \wedge \varepsilon^{a+1} e_{32} \wedge \varepsilon^{b} e_{21}$ is a boundary in $C_{*}\left(\mathfrak{g l}\left(k[\varepsilon]_{n}\right)\right)_{\mathfrak{g l}(\mathbb{Q})}$, since this element corresponds to the element $\left(1, \varepsilon^{a+1}, \varepsilon^{b}\right)$ in the Connes complex and $d\left(1, \varepsilon^{a+1}, \varepsilon^{b}, 1\right)=\left(1, \varepsilon^{a+1}, \varepsilon^{b}\right)$.

Therefore since $\beta_{w}$ is a cycle, so is $\gamma_{w}$, and to prove the lemma it suffices to show that $\left(\mathrm{Li}_{2, n, w} \circ T^{\prime}\right)\left(\varepsilon^{a} e_{12} \wedge \varepsilon^{b} e_{21} \wedge \varepsilon e_{33}\right)=0$ for $a+b \geq n$.

Note that since

$$
\begin{aligned}
d\left(e_{12} \wedge \varepsilon^{a} e_{11} \wedge \varepsilon^{b} e_{21} \wedge \varepsilon e_{33}\right)=-\varepsilon^{a} e_{12} \wedge \varepsilon^{b} e_{21} \wedge \varepsilon e_{33} \\
+\varepsilon^{a} e_{11} \wedge \varepsilon^{b} e_{11} \wedge \varepsilon e_{33}-\varepsilon^{a} e_{11} \wedge \varepsilon^{b} e_{22} \wedge \varepsilon e_{33},
\end{aligned}
$$

it is sufficient to show the vanishing of both

$$
\begin{aligned}
& \left(\mathrm{Li}_{2, n, w} \circ T^{\prime}\right)\left(\varepsilon^{a} e_{11} \wedge \varepsilon^{b} e_{11} \wedge \varepsilon e_{33}\right)=\left(\mathrm{Li}_{2, n, w} \circ T^{\prime \prime}\right)\left(\varepsilon^{a} e_{11} \wedge \varepsilon^{b} e_{11} \wedge \varepsilon e_{33}\right), \\
& \left(\mathrm{Li}_{2, n, w} \circ T^{\prime}\right)\left(\varepsilon^{a} e_{11} \wedge \varepsilon^{b} e_{22} \wedge \varepsilon e_{33}\right)=\left(\operatorname{Li}_{2, n, w} \circ T^{\prime \prime}\right)\left(\varepsilon^{a} e_{11} \wedge \varepsilon^{b} e_{22} \wedge \varepsilon e_{33}\right) .
\end{aligned}
$$

The equalities above follow immediately from the fact that $\varepsilon^{a} e_{11} \wedge \varepsilon^{b} e_{11} \wedge \varepsilon e_{33}$ and $\varepsilon^{a} e_{11} \wedge \varepsilon^{b} e_{22} \wedge \varepsilon e_{33}$ are cycles not only in $C_{*}\left(\mathfrak{g l}_{3}\left(k[\varepsilon]_{n}\right), \mathbb{Q}\right)_{\mathfrak{g r}_{3}(\mathbb{Q})}$ but also in $C_{*}\left(\mathfrak{g l}_{3}\left(k[\varepsilon]_{n}\right), \mathbb{Q}\right)$.

Propositions 2.2.1 and 2.2.2 give that $\mathrm{Li}_{2, n, w}(x)=\left(\mathrm{li}_{2,(2 n-1, n), w} \circ \delta_{2 n-1}\right)(\tilde{x})$ for $x \in B_{2}\left(k[\varepsilon]_{n}\right)$, where $\tilde{x} \in B_{2}\left(k[\varepsilon]_{2 n-1}\right)$ is any lift of $x$.

Let $\tilde{\alpha} \in\left\{\varepsilon^{a} e_{11} \wedge \varepsilon^{b} e_{11} \wedge \varepsilon e_{33}, \varepsilon^{a} e_{11} \wedge \varepsilon^{b} e_{22} \wedge \varepsilon e_{33}\right\} \subseteq C_{3}\left(\mathfrak{g l}\left(k[\varepsilon]_{2 n-1}\right), \mathbb{Q}\right)$, and let $\alpha$ the reduction of $\tilde{\alpha}$ to $C_{3}\left(\mathfrak{g l}\left(k[\varepsilon]_{n}\right), \mathbb{Q}\right)$. Then

$$
\operatorname{Li}_{2, n, w}\left(T^{\prime \prime}(\alpha)\right)=\left(\operatorname{li}_{2,(2 n-1, n), w} \circ \delta_{2 n-1}\right)\left(\underline{T}^{\prime \prime}(\tilde{\alpha})\right) .
$$

Here $\underline{T}^{\prime \prime}$ denotes the chain map, mapping $C_{3}\left(\mathfrak{g l}_{3}\left(k[\varepsilon]_{2 n-1}\right), \mathbb{Q}\right)$ to $B_{2}\left(k[\varepsilon]_{2 n-1}\right)_{\mathbb{Q}}$ and $C_{2}\left(\mathfrak{g l}_{3}\left(k[\varepsilon]_{2 n-1}\right), \mathbb{Q}\right)$ to $\bigwedge^{2} V_{2 n-1}$, that induces $T^{\prime \prime}$. The map $\underline{T}^{\prime \prime}$ depends on certain choices (see the next paragraph).

Recall how $\underline{T}^{\prime \prime}(\tilde{\alpha})$ is defined in Section 3: Through the antisymmetrization map $\alpha_{\text {as }}$ (Section 3.4) and the exponential map [Loday 1992, Sections 3.5 and 11.3.13], we get a chain map $C_{*}\left(\mathfrak{g l}_{3}\left(k[\varepsilon]_{2 n-1}\right), \mathbb{Q}\right)^{\circ} \rightarrow C_{*}\left(\hat{U}\left(\mathrm{GL}_{3}\left(k[\varepsilon]_{2 n-1}\right)\right), \mathbb{Q}\right)$. In fact, it is immediately seen that the image of $\tilde{\alpha}$ under these maps lies inside the image of $C_{*}\left(U\left(\mathrm{GL}_{3}\left(k[\varepsilon]_{2 n-1}\right)\right), \mathbb{Q}\right)$ in $C_{*}\left(\hat{U}\left(\mathrm{GL}_{3}\left(k[\varepsilon]_{2 n-1}\right)\right), \mathbb{Q}\right)$. To get from $C_{*}\left(U\left(\mathrm{GL}_{3}\left(k[\varepsilon]_{2 n-1}\right)\right), \mathbb{Q}\right)$ to $C_{*}\left(\mathrm{GL}_{3}\left(k[\varepsilon]_{2 n-1}\right), \mathbb{Q}\right)$ we pass via the map described in Section 3.6. Bypassing the need for stabilization since we are already in $\mathrm{GL}_{3}$, and using that $\tilde{D}$ is an acyclic complex of $\mathrm{GL}_{3}\left(k[\varepsilon]_{2 n-1}\right)$ modules, we get a (noncanonical) map from $C_{*}\left(\mathrm{GL}_{3}\left(k[\varepsilon]_{2 n-1}\right), \mathbb{Q}\right)$ to $\tilde{D}$. Finally taking $\mathrm{GL}_{3}\left(k[\varepsilon]_{2 n-1}\right)$ 
coinvariants, we end up in the complex $D$, and using the map of double complexes (induced by (3.8.2)), we pass from $D$ to the complex

$$
\gamma_{k[\varepsilon]_{2 n-1}}(2)_{\mathbb{Q}}: B_{2}\left(k[\varepsilon]_{2 n-1}\right)_{\mathbb{Q}} \stackrel{\delta_{2 n-1}}{\longrightarrow} \bigwedge^{2} V_{2 n-1}
$$

Since $\underline{T}^{\prime \prime}$ is a map of complexes, $\delta_{2 n-1}\left(\underline{T}^{\prime \prime}(\tilde{\alpha})\right)=\underline{T}^{\prime \prime}(d(\tilde{\alpha}))=0$, as $d(\tilde{\alpha})=0$ in $C_{*}\left(\mathfrak{g l}_{3}\left(k[\varepsilon]_{2 n-1}\right), \mathbb{Q}\right)$. This implies that

$$
\operatorname{Li}_{2, n, w}\left(T^{\prime \prime}(\alpha)\right)=\operatorname{li}_{2,(2 n-1, n), w}\left(\delta_{2 n-1}\left(T^{\prime \prime}(\tilde{\alpha})\right)\right)=0 .
$$

The next lemma will help us to reduce the computation to $\mathfrak{g l}_{2}$ :

Lemma 4.2.2. The chain $\gamma_{w}$ as defined above is a cycle in $C_{3}\left(\mathfrak{g l}_{2}\left(k[\varepsilon]_{n}\right), \mathbb{Q}\right)_{\mathfrak{g} \mathfrak{g}_{2}(\mathbb{Q})}$

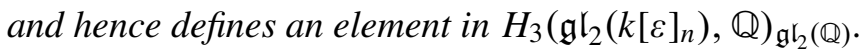

Proof. We already know that $\gamma_{w}$ defines a cycle in $C_{3}\left(\mathfrak{g l}_{3}\left(k[\varepsilon]_{n}\right), \mathbb{Q}\right)_{\mathfrak{g l}}(\mathbb{Q})$. Since $C_{i}\left(\mathfrak{g l}_{m}\left(k[\varepsilon]_{n}\right), \mathbb{Q}\right)_{\mathfrak{g l}_{m}(\mathbb{Q})}=\left(\bigwedge^{i} \mathfrak{g l}_{m}\left(k[\varepsilon]_{n}\right)\right)_{\mathfrak{g l}_{m}(\mathbb{Q})}($ see Section 3.2.2) and

$$
\left(\bigwedge^{i} \mathfrak{g l}_{m}\left(k[\varepsilon]_{n}\right)\right)_{\mathfrak{g l}_{m}(\mathbb{Q})}=\left(\bigwedge^{i} \mathfrak{g l}_{i}\left(k[\varepsilon]_{n}\right)\right)_{\mathfrak{g l}_{i}(\mathbb{Q})} \quad \text { for } m \geq i
$$

[Loday 1992, Corollary 9.2.8 and (10.2.10.1)], we have

$$
d\left(\gamma_{w}\right)=0 \in C_{2}\left(\mathfrak{g l}_{3}\left(k[\varepsilon]_{n}\right), \mathbb{Q}\right)_{\mathfrak{g l}_{3}(\mathbb{Q})}=C_{2}\left(\mathfrak{g l}_{2}\left(k[\varepsilon]_{n}\right), \mathbb{Q}\right)_{\mathfrak{g l}_{2}(\mathbb{Q})} .
$$

4.2.2. From $C_{*}\left(\mathfrak{g l}_{2}\left(k[\varepsilon]_{2 n-1}\right), \mathbb{Q}\right)_{\mathfrak{g l}}(\mathbb{Q})$ to $C_{*}\left(\mathfrak{g l}_{2}\left(k[\varepsilon]_{2 n-1}\right), \mathbb{Q}\right)$. In order to continue with the computation of $\operatorname{Li}_{2, n, w}\left(T^{\prime}\left(\gamma_{w}\right)\right)$, we need to compute the image of $\gamma_{w}$ in $C_{3}\left(\mathfrak{g l}_{2}\left(k[\varepsilon]_{n}, \mathbb{Q}\right)\right.$. This would be a very long computation, but in fact we will see in this section that we can get away with much less. The following proposition will be crucial.

Proposition 4.2.3. For any $\mathbb{Q}$-algebra $A$, let $\mathfrak{g l}_{n}(\mathbb{Q})$ act on $\mathfrak{g l}_{n}(A)$ by its adjoint action. Let $C_{*}^{\prime}\left(\mathfrak{g l}_{n}(A), \mathbb{Q}\right)_{\mathfrak{g l}}(\mathbb{Q})$ be the subcomplex of $C_{*}\left(\mathfrak{g l}_{n}(A), \mathbb{Q}\right)$ on which $\mathfrak{g l}_{n}(\mathbb{Q})$ acts trivially. Then the canonical map

$$
C_{*}^{\prime}\left(\mathfrak{g l}_{n}(A), \mathbb{Q}\right)_{\mathfrak{g l}_{n}(\mathbb{Q})} \rightarrow C_{*}\left(\mathfrak{g l}_{n}(A), \mathbb{Q}\right) \rightarrow C_{*}\left(\mathfrak{g l}_{n}(A), \mathbb{Q}\right)_{\mathfrak{g l}_{n}(\mathbb{Q})}
$$

is an isomorphism and there is a canonical direct sum of complexes

$$
C_{*}\left(\mathfrak{g l}_{n}(A), \mathbb{Q}\right)=C_{*}^{\prime}\left(\mathfrak{g l}_{n}(A), \mathbb{Q}\right)_{\mathfrak{g l}_{n}(\mathbb{Q})} \oplus L_{*},
$$

with $\mathfrak{g l}_{n}(\mathbb{Q})$-action, such that $L_{*}$ is acyclic.

Proof. This is [Loday 1992, Proposition 10.1.8], taking $\mathfrak{g}=\mathfrak{g l}_{n}(A)$ and $\mathfrak{h}=\mathfrak{g l}_{n}(\mathbb{Q})$, and noting the reductivity of $\mathfrak{g l}_{n}(\mathbb{Q})$ [loc. cit., 10.2.9].

To continue, we need to compute the image $\gamma_{w}^{\prime}$ of $\gamma_{w}$ in $H_{3}\left(\mathfrak{g l}_{2}\left(k[\varepsilon]_{n}\right), \mathbb{Q}\right)$. Then we should lift $\gamma_{w}^{\prime}$ to a chain $\tilde{\gamma}_{w}^{\prime}$ in $C_{3}^{\prime}\left(\mathfrak{g l}_{2}\left(k[\varepsilon]_{2 n-1}\right), \mathbb{Q}\right)_{\mathfrak{g l}}(\mathbb{Q})$ and continue 
just as in the last part of the proof of Lemma 4.2.1. Namely,

$$
\begin{aligned}
\operatorname{Li}_{2, n, w}\left(T^{\prime}\left(\gamma_{w}\right)\right) & =\operatorname{Li}_{2, n, w}\left(\underline{T}^{\prime \prime}\left(\tilde{\gamma}_{w}^{\prime}\right)\right)=\operatorname{li}_{2,(2 n-1, n), w}\left(\delta_{2 n-1}\left(\underline{T}^{\prime \prime}\left(\tilde{\gamma}_{w}^{\prime}\right)\right)\right) \\
& =\operatorname{li}_{2,(2 n-1, n), w}\left(\underline{T}^{\prime \prime}\left(d\left(\tilde{\gamma}_{w}^{\prime}\right)\right)\right) .
\end{aligned}
$$

Let $\tilde{\gamma}_{w}^{*}$ be any chain in $C_{3}\left(\mathfrak{g l}_{2}\left(k[\varepsilon]_{2 n-1}\right), \mathbb{Q}\right)$ that has a cycle for its image in $C_{3}\left(\mathfrak{g l}_{2}\left(k[\varepsilon]_{n}\right), \mathbb{Q}\right)_{\mathfrak{g l}_{2}(\mathbb{Q})}$ (under the canonical maps) and that lifts $\gamma_{w}$. Then, by Proposition 4.2 .3 , the first component $\tilde{\gamma}_{w}^{*(1)}$ of $\tilde{\gamma}_{w}^{*}$ under the decomposition in (4.2.1) is a lift of the element $\gamma_{w}^{\prime}$. Therefore we can choose $\tilde{\gamma}_{w}^{\prime}:=\tilde{\gamma}_{w}^{*(1)}$, and to continue we need to compute $d\left(\tilde{\gamma}_{w}^{*(1)}\right)=d\left(\tilde{\gamma}_{w}^{*}\right)^{(1)}$.

For the rest of the computation, we will let $\tilde{\gamma}_{w}^{*}:=\tilde{\gamma}_{w}$, where

$$
\begin{aligned}
\tilde{\gamma}_{w} & :=\sum_{0 \leq j<(2 n-1-w) / 2} \tilde{\gamma}_{n-1-j, w-n+j}+\frac{1}{2} \chi(w) \tilde{\gamma}_{(w-1) / 2,(w-1) / 2}, \\
\tilde{\gamma}_{a, b} & :=\varepsilon^{a} e_{12} \wedge \varepsilon^{b} e_{21} \wedge \varepsilon e_{11} .
\end{aligned}
$$

Combining the above we have

$$
\operatorname{Li}_{2, n, w}\left(T^{\prime}\left(\gamma_{w}\right)\right)=\operatorname{li}_{2,(2 n-1, n), w}\left(\underline{T}^{\prime \prime}\left(d\left(\tilde{\gamma}_{w}\right)^{(1)}\right)\right) .
$$

Next we will compute $d\left(\tilde{\gamma}_{a, b}\right)^{(1)}$. For any $\mathbb{Q}$-algebra $A$ there is a canonical isomorphism for $i \geq n$ given by

$$
C_{n}\left(\mathfrak{g l}_{i}(A), \mathbb{Q}\right)_{\mathfrak{g l}_{i}(\mathbb{Q})}=\left(\bigwedge^{n} \mathfrak{g l}_{i}(A)\right)_{\mathfrak{g l}_{i}(\mathbb{Q})} \rightarrow\left(\mathbb{Q}\left[S_{n}\right] \otimes A^{\otimes n}\right)_{S_{n}},
$$

where $S_{n}$ acts on $\mathbb{Q}\left[S_{n}\right]$ by conjugation and on $A^{\otimes n}$ by permuting the factors and multiplying with sign [Loday 1992, 10.2.10.1].

Letting

$$
\Gamma_{x, y}:=x e_{12} \wedge y e_{21}+x e_{21} \wedge y e_{12}+\frac{1}{2} x\left(e_{22}-e_{11}\right) \wedge y\left(e_{22}-e_{11}\right) \text { for } x, y \in A,
$$

we see by direct computation that $\Gamma_{x, y} \in C_{2}^{\prime}\left(\mathfrak{g l}_{2}(A), \mathbb{Q}\right)_{\mathfrak{g l}}(\mathbb{Q})$.

Under the map (4.2.3),

$$
\begin{aligned}
\Gamma_{x, y} \mapsto(3 \cdot \tau) \otimes(x \otimes y), \quad x\left(e_{11}-e_{22}\right) \wedge y e_{11} & \mapsto(1 \cdot \tau) \otimes(x \otimes y), \\
x e_{21} \wedge y e_{12} & \mapsto(1 \cdot \tau) \otimes(x \otimes y), \\
x e_{12} \wedge y e_{21} & \mapsto(1 \cdot \tau) \otimes(x \otimes y),
\end{aligned}
$$

where $S_{2}=\{\mathrm{id}, \tau\}$. Therefore, using Proposition 4.2.3, we have

$$
\left(x\left(e_{11}-e_{22}\right) \wedge y e_{11}\right)^{(1)}=\left(x e_{21} \wedge y e_{12}\right)^{(1)}=\left(x e_{12} \wedge y e_{21}\right)^{(1)}=\frac{1}{3} \Gamma_{x, y} .
$$

Since $d\left(\tilde{\gamma}_{a, b}\right)=\varepsilon^{a+b}\left(e_{11}-e_{22}\right) \wedge \varepsilon e_{11}-\varepsilon^{b} e_{21} \wedge \varepsilon^{a+1} e_{12}-\varepsilon^{a} e_{12} \wedge \varepsilon^{b+1} e_{21}$, we have

$$
d\left(\tilde{\gamma}_{a, b}\right)^{(1)}=\frac{1}{3}\left(\Gamma_{\varepsilon^{a+b}, \varepsilon}-\Gamma_{\varepsilon^{b}, \varepsilon^{a+1}}-\Gamma_{\varepsilon^{a}, \varepsilon^{b+1}}\right) .
$$


4.2.3. Fixing a choice for $\underline{T}^{\prime \prime}$. We need to fix a choice for the restriction of $\underline{T}^{\prime \prime}$ to $C_{*}\left(\mathfrak{g l}_{2}\left(k[\varepsilon]_{2 n-1}\right), \mathbb{Q}\right)$. So, recalling the last part of the proof of Lemma 4.2.1, we need to fix the map from $C_{*}\left(\mathrm{GL}_{2}\left(k[\varepsilon]_{2 n-1}\right), \mathbb{Q}\right) \rightarrow \tilde{D} \rightarrow D \rightarrow \gamma_{k[\varepsilon]_{2 n-1}}(2)_{\mathbb{Q}}$, in degree 2.

Fixing $v_{1}, v_{2}, v_{3}$ any three vectors in $k[\varepsilon]_{2 n-1}^{\oplus 2}$ in general position, we define a map that sends $\left(g_{1}, g_{2}, g_{3}\right) \in C_{2}\left(\mathrm{GL}_{2}\left(k[\varepsilon]_{2 n-1}\right), \mathbb{Q}\right)$ to

$$
\begin{aligned}
\left(w, g_{1} v_{1}, g_{2} v_{2}, g_{3} v_{3}\right)-\left(w, g_{1} v_{1},\right. & \left.g_{2} v_{2}, g_{2} v_{3}\right) \\
& -\left(w, g_{1} v_{1}, g_{1} v_{2}, g_{3} v_{3}\right)+\left(w, g_{1} v_{1}, g_{1} v_{2}, g_{2} v_{3}\right)
\end{aligned}
$$

in $\tilde{C}_{4}\left(k[\varepsilon]_{2 n-1}^{\oplus 3}\right)=\tilde{C}_{4}(3) \subseteq \tilde{C}_{4}(3) \oplus \tilde{C}_{3}(3)$, where we view

$$
k[\varepsilon]_{2 n-1}^{\oplus 2}=\left\{\left(a_{1}, a_{2}, a_{3}\right) \in k[\varepsilon]_{2 n-1}^{\oplus 3} \mid a_{3}=0\right\},
$$

and we let $w=(0,0,1)$. It is seen without difficulty that this map can be extended to a map of complexes $C_{*}\left(\mathrm{GL}_{2}\left(k[\varepsilon]_{2 n-1}\right), \mathbb{Q}\right) \rightarrow \tilde{D}$.

Composing with the remaining map given in (3.8.2) this gives a map that sends $\left(g_{1}, g_{2}, g_{3}\right)$ to

$\beta\left(\left(g_{1} v_{1}, g_{2} v_{2}, g_{3} v_{3}\right)-\left(g_{1} v_{1}, g_{2} v_{2}, g_{2} v_{3}\right)-\left(g_{1} v_{1}, g_{1} v_{2}, g_{3} v_{3}\right)+\left(g_{1} v_{1}, g_{1} v_{2}, g_{2} v_{3}\right)\right)$

in $\bigwedge^{2} V_{2 n-1}$, where $\beta$ is the map in (3.8.3). From now on we fix $v_{1}:=(1,1)$, $v_{2}:=(0,1)$ and $v_{3}:=(1,0)$ and denote the resulting map by $\underline{T}^{\prime \prime}$.

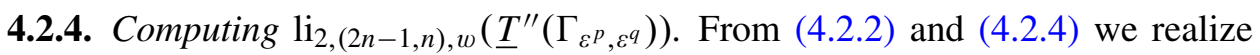
that we need to compute $\mathrm{li}_{2,(2 n-1, n), w}\left(\underline{T}^{\prime \prime}\left(\Gamma_{\varepsilon^{p}, \varepsilon^{q}}\right)\right)$ for $p+q=w$. We will do this in a few steps.

Lemma 4.2.4. For $i=1,2$ and $p+q=w$, with $p, q \geq 1$, we have

$$
\operatorname{li}_{2,(2 n-1, n), w}\left(\underline{T}^{\prime \prime}\left(\varepsilon^{p} e_{i i} \wedge \varepsilon^{q} e_{i i}\right)\right)=0 .
$$

Proof. The element $\varepsilon^{p} e_{i i} \wedge \varepsilon^{q} e_{i i}$ maps to

$$
\varepsilon^{p} e_{i i} \otimes \varepsilon^{q} e_{i i}-\varepsilon^{q} e_{i i} \otimes \varepsilon^{p} e_{i i} \in C_{2}\left(u\left(\mathfrak{g l}_{2}\left(k[\varepsilon]_{2 n-1}\right)\right), \mathbb{Q}\right) .
$$

Since $\varepsilon^{x} e_{i i}=\log \left(1-\left(1-\exp \left(\varepsilon^{x} e_{i i}\right)\right)\right)=-\sum_{1 \leq k}\left(1-\exp \left(\varepsilon^{x} e_{i i}\right)\right)^{k} / k$ for $x \geq 1$, we see that $\varepsilon^{p} e_{i i} \otimes \varepsilon^{q} e_{i i}$ is a $\mathbb{Q}$-linear combination of terms of the form

$$
\exp \left(\varepsilon^{s} e_{i i}\right)^{u} \otimes \exp \left(\varepsilon^{t} e_{i i}\right)^{v} .
$$

Let $g_{1}:=\exp \left(\varepsilon^{s} e_{i i}\right)^{u}$ and $g_{2}:=\exp \left(\varepsilon^{t} e_{i i}\right)^{v}$. Then $g_{1} \otimes g_{2}$ maps to $\left(1, g_{1}, g_{1} g_{2}\right)$, which maps to

$$
\left(v_{1}, g_{1} v_{2}, g_{1} g_{2} v_{3}\right)-\left(v_{1}, g_{1} v_{2}, g_{1} v_{3}\right)-\left(v_{1}, v_{2}, g_{1} g_{2} v_{3}\right)+\left(v_{1}, v_{2}, g_{1} v_{3}\right) \text {. }
$$

Since, depending on $i, g_{1}\left(v_{2}\right)=v_{2}$ or $g_{1}\left(v_{3}\right)=g_{1} g_{2}\left(v_{3}\right)=v_{3}$, we see that the last expression is 0 . 
Lemma 4.2.5. The value of $\mathrm{li}_{2,(2 n-1, n), w}$ on the image of the element $\varepsilon^{p} e_{i j} \otimes \varepsilon^{q} e_{k l}$ in $\bigwedge^{2} V_{2 n-1}$, under the chain map that we fixed in Section 4.2.3, is 0 if $p+q \neq w$ and $p, q \geq 1$.

Proof. By Proposition 2.1.2 to compute the value of $\mathrm{li}_{2,(2 n-1, n), w}$ on the image of $\varepsilon^{p} e_{i j} \otimes \varepsilon^{q} e_{k l}$ in $\bigwedge^{2} V_{2 n-1}$, we first need to project that image to $S_{k}(2 n-1, n)_{\langle w\rangle}$. But for $\lambda \in \mathbb{Q}$, replacing $\varepsilon$ with $\lambda \varepsilon$ multiplies $\varepsilon^{p} e_{i j} \otimes \varepsilon^{q} e_{k l}$ by $\lambda^{p+q}$, whereas the projection of its image to $S_{k}(2 n-1, n)_{\langle w\rangle}$ gets multiplied by $\lambda^{w}$. Therefore this projection is 0 . Hence the statement in the lemma.

Lemma 4.2.6. For $p+q=w$ with $p, q \geq 1$, we have

$$
\operatorname{li}_{2,(2 n-1, n), w}\left(\underline{T}^{\prime \prime}\left(\varepsilon^{p} e_{22} \wedge \varepsilon^{q} e_{11}\right)\right)=\operatorname{li}_{2,(2 n-1, n), w}\left(\left(1+\varepsilon^{q}\right) \wedge\left(1+\varepsilon^{p}\right)\right) .
$$

Proof. The expression $\varepsilon^{p} e_{22} \wedge \varepsilon^{q} e_{11}$ maps to

$$
\varepsilon^{p} e_{22} \otimes \varepsilon^{q} e_{11}-\varepsilon^{q} e_{11} \otimes \varepsilon^{p} e_{22} .
$$

Both $\varepsilon^{p} e_{i i} \otimes \varepsilon^{q} e_{j j}$ and $\exp \left(\varepsilon^{p} e_{i i}\right) \otimes \exp \left(\varepsilon^{q} e_{j j}\right)-\exp \left(\varepsilon^{p} e_{i i}\right) \otimes 1-1 \otimes \exp \left(\varepsilon^{q} e_{j j}\right)$ have the same $k^{\times}$-weight $w$ component, and therefore by Lemma 4.2 .5 have the same image. Note that terms of the form $1 \otimes g$ and $g \otimes 1$ map to 0 , because of the computation in (4.2.5). Hence the left side of the expression in the lemma is equal to the image of $\exp \left(\varepsilon^{p} e_{22}\right) \otimes \exp \left(\varepsilon^{q} e_{11}\right)-\exp \left(\varepsilon^{q} e_{11}\right) \otimes \exp \left(\varepsilon^{p} e_{22}\right)$. Since $\exp \left(\varepsilon^{q} e_{11}\right) v_{2}=v_{2}$, using the expression (4.2.5) we see that $\exp \left(\varepsilon^{q} e_{11}\right) \otimes \exp \left(\varepsilon^{p} e_{22}\right)$ maps to 0. Again using (4.2.5) and the definition of $\beta$ and $\operatorname{li}_{2,(2 n-1, n), w}$, we see that $\exp \left(\varepsilon^{p} e_{22}\right) \otimes \exp \left(\varepsilon^{q} e_{11}\right)$ maps to $\operatorname{li}_{2,(2 n-1, n), w}\left(\left(1+\varepsilon^{q}\right) \wedge\left(1+\varepsilon^{p}\right)\right)$.

Lemma 4.2.7. For $p+q=w$ with $p, q \geq 1$, we have

$$
\operatorname{li}_{2,(2 n-1, n), w}\left(\underline{T}^{\prime \prime}\left(\varepsilon^{p} e_{12} \wedge \varepsilon^{q} e_{21}\right)\right)=\operatorname{li}_{2,(2 n-1, n), w}\left(\left(1-\varepsilon^{p}\right) \wedge\left(1-\varepsilon^{q}\right)\right) .
$$

Proof. Exactly as in the proof of Lemma 4.2.6, we see that the left side of the expression above is equal to the image of

$$
\exp \left(\varepsilon^{p} e_{12}\right) \otimes \exp \left(\varepsilon^{q} e_{21}\right)-\exp \left(\varepsilon^{q} e_{21}\right) \otimes \exp \left(\varepsilon^{p} e_{12}\right) .
$$

As $\exp \left(\varepsilon^{q} e_{21}\right)\left(v_{2}\right)=v_{2}$, we see, using (4.2.5), that $\exp \left(\varepsilon^{q} e_{21}\right) \otimes \exp \left(\varepsilon^{p} e_{12}\right)$ maps to 0 . Finally using (4.2.5), and the definition of $\beta$ and $\operatorname{li}_{2,(2 n-1, n), w}$ we see that $\exp \left(\varepsilon^{p} e_{12}\right) \otimes \exp \left(\varepsilon^{q} e_{21}\right)$ maps to $\operatorname{li}_{2,(2 n-1, n), w}\left(\left(1-\varepsilon^{p}\right) \wedge\left(1-\varepsilon^{q}\right)\right)$.

Lemma 4.2.8. For $p+q=w$ with $p, q \geq 1$,

$$
\operatorname{li}_{2,(2 n-1, n), w}\left(\underline{T}^{\prime \prime}\left(\Gamma_{\varepsilon^{p}, \varepsilon^{q}}\right)\right)=3 \operatorname{li}_{2,(2 n-1, n), w}\left(\left(1-\varepsilon^{p}\right) \wedge\left(1-\varepsilon^{q}\right)\right) .
$$

Proof. This follows from Lemmas 4.2.4, 4.2.6, and 4.2.7, together with the fact, which is immediate from the definition of $\operatorname{li}_{2,(2 n-1, n), w}$, that

$$
\operatorname{li}_{2,(2 n-1, n), w}\left(\left(1-\varepsilon^{p}\right) \wedge\left(1-\varepsilon^{q}\right)\right)=\operatorname{li}_{2,(2 n-1, n), w}\left(\left(1+\varepsilon^{p}\right) \wedge\left(1+\varepsilon^{q}\right)\right) .
$$


Let $[|\cdot|]$ denote the greatest integer function.

Theorem 4.2.9. With the notation as in Section 4.1.2,

$$
\operatorname{Li}_{2, n}\left(T\left(\alpha_{w}\right)\right)= \begin{cases}-\left(\left[\left|\frac{1}{2}(2 n-1-w)\right|\right]+w-n+1+\frac{1}{2} \chi(w)\right) & \text { if } w \neq 2 n-1, \\ -\frac{1}{2}(2 n-1) & \text { if } w=2 n-1 .\end{cases}
$$

Proof. Since $\operatorname{Li}_{2, n}\left(T\left(\alpha_{w}\right)\right)=\operatorname{Li}_{2, n, w}\left(T^{\prime}\left(\beta_{w}\right)\right)$, using Lemma 4.2.1, (4.2.2), (4.2.4) we see that $\operatorname{Li}_{2, n}\left(T\left(\alpha_{w}\right)\right)=\frac{1}{2} \operatorname{li}_{2,(2 n-1, n), w} \circ \underline{T}^{\prime \prime}$ evaluated on

$\sum_{0 \leq j<(2 n-1-w) / 2}\left(\Gamma_{\varepsilon^{w-1}, \varepsilon}-\Gamma_{\varepsilon^{w-n+j}, \varepsilon^{n-j}}-\Gamma_{\varepsilon^{n-1-j}, \varepsilon^{w-n+j+1}}\right)$

$$
+\frac{1}{2} \chi(w)\left(\Gamma_{\varepsilon^{w-1}, \varepsilon}-2 \Gamma_{\varepsilon^{(w-1) / 2}, \varepsilon^{(w+1) / 2}}\right) .
$$

Using Lemma 4.2.8 and the definition of $\operatorname{li}_{2,(2 n-1, n), w}$ we see that if $w \neq 2 n-1$, then the contribution from $j=0$ is $-(w-n+1)$; the contribution from each of the terms where $0<j$ is -1 ; the last term contributes $-\frac{1}{2} \chi(w)$.

In the case $w=2 n-1$, there is only one contribution, coming from the last term, and this is $\frac{1}{2} \chi(2 n-1)(-1-2(n-1))=-\frac{1}{2}(2 n-1)$.

4.3. Proof of Theorem 1.3.2. In order to prove this, by Goodwillie's theorem (Theorem 3.1.1), Remark 3.1.2 and Sections 4.1.1 and 4.1.2, we need only show

$$
\mathrm{Li}_{2, n, w}:(k \simeq) \mathrm{HC}_{2}\left(k[\varepsilon]_{n},(\varepsilon)\right)_{\langle w\rangle}^{(1)} \rightarrow k
$$

is an isomorphism. We know that this map is nonzero by Theorem 4.2.9, and replacing $\varepsilon$ by $\lambda \varepsilon$ has the effect of multiplication by $\lambda^{w}$, using the vector space structures on both sides [Hesselholt 2005, Proposition 8.1]. This immediately implies the theorem when $k$ is algebraically closed. In the general case, we just need to use Theorem 1.3.2 for $\bar{k}$, and the equivariance of $\operatorname{Li}_{2, n, w}$ with respect to $\operatorname{Gal}(\bar{k} / k)$ and take galois invariants on both sides.

\section{The complex $\gamma_{k[\varepsilon]_{n}}(2)_{\mathbb{Q}}$}

5.1. To compute the kernel of $\delta_{n}$ in Theorem 1.3.1, we will need the following proposition. Following Suslin's notation, let $T_{m}(A) \subseteq \mathrm{GL}_{m}(A)$ denote the subgroup of diagonal matrices.

Proposition 5.1.1. The map $\rho_{2}: H_{3}\left(\mathrm{GL}_{3}\left(k[\varepsilon]_{n}\right), \mathbb{Q}\right) \rightarrow \operatorname{ker}\left(\delta_{n}\right)$ from Section 3.8 has the property that

$$
\rho_{2}\left(H_{3}\left(\mathrm{GL}_{2}\left(k[\varepsilon]_{n}\right), \mathbb{Q}\right)=\operatorname{ker}\left(\delta_{n}\right) \quad \text { and } \quad H_{3}\left(T_{3}\left(k[\varepsilon]_{n}\right), \mathbb{Q}\right) \subseteq \operatorname{ker}\left(\rho_{2}\right) .\right.
$$


Proof. The first statement is proved in the case of fields in [Suslin 1990, Section 2]. The same proof works for $k[\varepsilon]_{n}$, if in the first line of [Suslin 1990, page 222], we use [Guin 1989, Theorem 2.2.2] to show that

$$
H_{*}\left(T_{2}\left(k[\varepsilon]_{n}\right), \mathbb{Q}\right)=H_{*}\left(U T_{2}\left(k[\varepsilon]_{n}\right), \mathbb{Q}\right),
$$

where $U T_{2}(A)$ denotes upper triangular matrices in $\mathrm{GL}_{2}(A)$ (this is denoted by $B_{2}(A)$ in [Suslin 1990]). We note that there is a slight difference between the construction of our map $\rho_{2}$ and the corresponding map of Suslin. Namely, Suslin uses configurations in the projective space rather than the affine space, but the resulting maps $H_{3}\left(\mathrm{GL}_{3}\left(k[\varepsilon]_{n}\right), \mathbb{Q}\right) \rightarrow \operatorname{ker}\left(\delta_{n}\right)$ are the same.

The proof of [Suslin 1990, Proposition 3.1] works for $k[\varepsilon]_{n}$ as well, proving the second statement.

Proposition 5.1.2. The map $T: \mathrm{HC}_{2}\left(k[\varepsilon]_{n},(\varepsilon)\right)^{(1)} \rightarrow \operatorname{ker}\left(\delta_{n}\right)^{\circ}$ (see Notation 2.0.1) defined in Proposition 3.8.9 is surjective.

Proof. Because of Proposition 5.1.1, Theorem 3.1.1 and Remark 3.1.2, it suffices to show that the image of $K_{3}\left(k[\varepsilon]_{n}\right)_{\mathbb{Q}}^{(2)}$ in $H_{3}\left(\mathrm{GL}_{3}\left(k[\varepsilon]_{n}\right), \mathbb{Q}\right)^{\circ} / H_{3}\left(T_{3}\left(k[\varepsilon]_{n}\right), \mathbb{Q}\right)^{\circ}$, under the composition of the maps

$$
\begin{aligned}
K_{3}\left(k[\varepsilon]_{n}\right)_{\mathbb{Q}}^{(2)} & \rightarrow K_{3}\left(k[\varepsilon]_{n}\right)_{\mathbb{Q}} \rightarrow H_{3}\left(\mathrm{GL}\left(k[\varepsilon]_{n}\right), \mathbb{Q}\right) \simeq H_{3}\left(\mathrm{GL}_{3}\left(k[\varepsilon]_{n}\right), \mathbb{Q}\right) \\
& \rightarrow H_{3}\left(\mathrm{GL}_{3}\left(k[\varepsilon]_{n}\right), \mathbb{Q}\right)^{\circ} / H_{3}\left(T_{3}\left(k[\varepsilon]_{n}\right), \mathbb{Q}\right)^{\circ},
\end{aligned}
$$

contains that of $H_{3}\left(\mathrm{GL}_{2}\left(k[\varepsilon]_{n}\right), \mathbb{Q}\right)$ in $H_{3}\left(\mathrm{GL}_{3}\left(k[\varepsilon]_{n}\right), \mathbb{Q}\right)^{\circ} / H_{3}\left(T_{3}\left(k[\varepsilon]_{n}\right), \mathbb{Q}\right)^{\circ}$.

Let $\wedge V$ denote the graded symmetric algebra over a graded vector space $V$. By the Milnor-Moore theorem, $H_{*}(\mathrm{GL}(A), \mathbb{Q}) \simeq \bigwedge\left(\left(K_{*}(A)_{\mathbb{Q}}\right)_{>0}\right)$ [Loday 1992, Corollary 11.2.12]; by the stability theorem,

$$
H_{3}\left(\mathrm{GL}_{3}\left(k[\varepsilon]_{n}\right), \mathbb{Q}\right)=H_{3}\left(\mathrm{GL}\left(k[\varepsilon]_{n}\right), \mathbb{Q}\right)
$$

[Guin 1989, Section 2]. Combining these, we obtain $H_{3}\left(\mathrm{GL}_{3}\left(k[\varepsilon]_{n}\right), \mathbb{Q}\right)=\bigwedge^{3} K_{1}\left(k[\varepsilon]_{n}\right)_{\mathbb{Q}} \oplus\left(K_{1}\left(k[\varepsilon]_{n}\right)_{\mathbb{Q}} \otimes K_{2}\left(k[\varepsilon]_{n}\right)_{\mathbb{Q}}\right) \oplus K_{3}\left(k[\varepsilon]_{n}\right)_{\mathbb{Q}}$.

The first two components of the decomposition lie inside

$$
H_{1}\left(\mathrm{GL}_{1}\left(k[\varepsilon]_{n}\right), \mathbb{Q}\right) \otimes H_{2}\left(\mathrm{GL}_{2}\left(k[\varepsilon]_{n}\right), \mathbb{Q}\right) \subseteq H_{3}\left(T_{3}\left(k[\varepsilon]_{n}\right), \mathbb{Q}\right),
$$

(by the proof of [Suslin 1990, Lemma 4.2]; [Guin 1989]). Therefore it suffices to prove that the image of $K_{3}\left(k[\varepsilon]_{n}\right)_{\mathbb{Q}}^{(2)}$ under the canonical projection

$$
H_{3}\left(\mathrm{GL}_{3}\left(k[\varepsilon]_{n}\right), \mathbb{Q}\right) \rightarrow \operatorname{Prim} H_{3}\left(\mathrm{GL}_{3}\left(k[\varepsilon]_{n}\right), \mathbb{Q}\right) \rightarrow\left(\operatorname{Prim} H_{3}\left(\mathrm{GL}_{3}\left(k[\varepsilon]_{n}\right), \mathbb{Q}\right)\right)^{\circ}
$$

contains the image of $H_{3}\left(\mathrm{GL}_{2}\left(k[\varepsilon]_{n}\right), \mathbb{Q}\right)$. 
By the construction of $\rho_{1}$ in Sections 3.2-3.7 and Remark 3.1.2, the last translates to showing that the image $\operatorname{im}\left(H_{3}\left(\mathfrak{g l}_{2}\left(k[\varepsilon]_{n}\right), \mathbb{Q}\right)\right)$ of $H_{3}\left(\mathfrak{g l}_{2}\left(k[\varepsilon]_{n}\right), \mathbb{Q}\right)$ in

$\left(\operatorname{Prim} H_{3}\left(\mathfrak{g l}_{3}\left(k[\varepsilon]_{n}\right), \mathbb{Q}\right)\right)^{\circ}=\mathrm{HC}_{2}\left(k[\varepsilon]_{n}\right)^{\circ}=\mathrm{HC}_{2}\left(k[\varepsilon]_{n}\right)^{\circ(1)} \oplus \mathrm{HC}_{2}\left(k[\varepsilon]_{n}\right)^{\circ(2)}$

is contained in $\mathrm{HC}_{2}\left(k[\varepsilon]_{n}\right)^{\circ(1)}$.

First note that $\alpha_{w}$ for $n+1 \leq w \leq 2 n-1$ form a basis for $\operatorname{HC}_{2}\left(k[\varepsilon]_{n}\right)^{\circ(1)}$ by Theorem 4.2.9 and Section 4.1.1. By Lemmas 4.2.1 and 4.2.2 and Proposition 4.2.3 and the discussion following it, the image of $\alpha_{w}$ in $H_{3}\left(\mathfrak{g l}_{3}\left(k[\varepsilon]_{n}\right)\right)^{\circ}$ is equal to that of an element $\gamma_{w}^{\prime} \in H_{3}\left(\mathfrak{g l}_{2}\left(k[\varepsilon]_{n}\right)\right)^{\circ}$. This implies immediately that $\mathrm{HC}_{2}\left(k[\varepsilon]_{n}\right)^{\circ(1)} \subseteq \operatorname{im}\left(H_{3}\left(\mathfrak{g l}_{2}\left(k[\varepsilon]_{n}\right), \mathbb{Q}\right)\right)$.

On the other hand [Loday 1992, Theorems 10.3.4 and 4.6.8] and [Loday and Quillen 1984, Remark 6.10] imply that there is a natural map

$$
\left(\operatorname{Prim} H_{3}\left(\mathfrak{g l}_{3}\left(k[\varepsilon]_{n}\right), \mathbb{Q}\right)\right)^{\circ} / \operatorname{im}\left(H_{3}\left(\mathfrak{g l}_{2}\left(k[\varepsilon]_{n}\right), \mathbb{Q}\right)\right) \rightarrow \mathrm{HC}_{2}\left(k[\varepsilon]_{n}\right)^{\circ(2)}
$$

which induces an automorphism of $\mathrm{HC}_{2}\left(k[\varepsilon]_{n}\right)^{\circ(2)}$ when precomposed with

$$
\mathrm{HC}_{2}\left(k[\varepsilon]_{n}\right)^{\circ(2)} \rightarrow\left(\operatorname{Prim} H_{3}\left(\mathfrak{g l}_{3}\left(k[\varepsilon]_{n}\right), \mathbb{Q}\right)\right)^{\circ} / \operatorname{im}\left(H_{3}\left(\mathfrak{g l}_{2}\left(k[\varepsilon]_{n}\right), \mathbb{Q}\right)\right) .
$$

These imply that $\operatorname{im}\left(H_{3}\left(\mathfrak{g l}_{2}\left(k[\varepsilon]_{n}\right), \mathbb{Q}\right)\right)=\mathrm{HC}_{2}\left(k[\varepsilon]_{n}\right)^{\circ(1)}$.

The corollary below computes the infinitesimal part of the first cohomology of the complex $\gamma_{k[\varepsilon]_{n}}(2)_{\mathbb{Q}}$. Note that $H^{1}\left(\gamma_{k[\varepsilon]_{n}}(2)_{\mathbb{Q}}\right)^{\circ}=\operatorname{ker}\left(\delta_{n}\right)^{\circ}$.

Corollary 5.1.3. The maps

$$
T: \mathrm{HC}_{2}\left(k[\varepsilon]_{n},(\varepsilon)\right)^{(1)} \rightarrow \operatorname{ker}\left(\delta_{n}\right)^{\circ} \quad \text { and } \quad \operatorname{Li}_{2, n}: \operatorname{ker}\left(\delta_{n}\right)^{\circ} \rightarrow k^{\oplus n-1}
$$

are isomorphisms.

Proof. This follows from the fact that $T$ is surjective (Proposition 5.1.2) and that $\mathrm{Li}_{2, n} \circ T$ is an isomorphism (Theorem 1.3.2).

Proposition 5.1.4. There are natural isomorphisms

$$
\left.H^{2}\left(\gamma_{k[\varepsilon]_{n}}(2)_{\mathbb{Q}}\right)^{\circ} \simeq \mathrm{HC}_{1}\left(k[\varepsilon]_{n}\right)^{\circ}=\mathrm{HC}_{1}\left(k[\varepsilon]_{n}\right)\right)^{\circ(1)} \simeq \bigoplus_{1 \leq i \leq n-1} \Omega_{k}^{1} .
$$

Proof. Note that by the definition of Milnor K-theory [Loday 1992, 11.1.16]

$$
H^{2}\left(\gamma_{k[\varepsilon]_{n}}(2)_{\mathbb{Q}}\right)=K_{2}^{M}\left(k[\varepsilon]_{n}\right) .
$$

Since

$$
K_{2}^{M}\left(k[\varepsilon]_{n}\right)=K_{2}\left(k[\varepsilon]_{n}\right)
$$

[Guin 1989, Section 4.2], we have, by [Loday 1992, Proposition 2.1.14],

$$
K_{2}\left(k[\varepsilon]_{n}\right)^{\circ}=\mathrm{HC}_{1}\left(k[\varepsilon]_{n}\right)^{\circ}=\Omega_{k[\varepsilon]_{n}}^{1} /\left(\Omega_{k}^{1}+d\left(k[\varepsilon]_{n}\right)\right) \simeq \bigoplus_{1 \leq i \leq n-1} \Omega_{k}^{1} .
$$


Finally $\mathrm{HC}_{1}\left(k[\varepsilon]_{n}\right)=\mathrm{HC}_{1}\left(k[\varepsilon]_{n}\right)^{(1)}$ follows from [loc. cit., Theorem 4.6.7].

5.2. Proof of Theorem 1.3.1. Over $k$ this is the main theorem in [Suslin 1990]. However, note that there the indecomposable quotient $K_{3}(k)_{\text {ind, } \mathbb{Q}}$ of $K_{3}(k)_{\mathbb{Q}}$ appears instead of $K_{3}(k)_{\mathbb{Q}}^{(2)}$. To see that these two groups are canonically isomorphic, see [Lichtenbaum 1987, page 207]. Therefore we only need to compute the cohomology of the infinitesimal part of the complex $\gamma_{k[\varepsilon]_{n}}(2)_{\mathbb{Q}}$. And this is done in Corollary 5.1.3 and (5.1.1) and (5.1.2).

\section{Comparison with the additive dilogarithm of Bloch and Esnault}

In this section we compare the complex $\gamma_{k[\varepsilon]_{n}}(2)_{\mathbb{Q}}^{\circ}$ to the complex $T_{n} \mathbb{Q}(2)(k)$ of Bloch and Esnault.

6.1. The reduced complex. To make the comparison we first define a subcomplex of $\gamma_{k[\varepsilon]_{n}}(2)_{\mathbb{Q}}^{\circ}$ : Define $\gamma_{k[\varepsilon]_{n}}(2)_{\mathbb{Q}}^{\prime}$ to be the subcomplex of $\gamma_{k[\varepsilon]_{n}}(2)_{\mathbb{Q}}^{\circ}$ whose degree 2 term is

$$
k^{\times} \otimes V_{n}^{\circ} \subseteq\left(\bigwedge^{2} V_{n}\right)^{\circ}
$$

and whose degree 1 term is the inverse image $\delta_{n}^{-1}\left(k^{\times} \otimes V_{n}^{\circ}\right) \subseteq B_{2}\left(k[\varepsilon]_{n}\right)_{\mathbb{Q}}^{\circ}$. Denote this last group by $B_{2}\left(k[\varepsilon]_{n}\right)_{\mathbb{Q}}^{\prime}$. Then we have

$$
\gamma_{k[\varepsilon]_{n}}(2)_{\mathbb{Q}}^{\prime}: B_{2}\left(k[\varepsilon]_{n}\right)_{\mathbb{Q}}^{\prime} \rightarrow k^{\times} \otimes V_{n}^{\circ} .
$$

We need a lemma to compute the cohomology of this reduced complex.

Lemma 6.1.1. The natural map $\left(k^{\times}\right)^{\otimes(i-1)} \otimes k[\varepsilon]_{n}^{\times} \rightarrow K_{i}^{M}\left(k[\varepsilon]_{n}\right)$ is a surjection. Proof. By the definition of Milnor K-theory, it is clear that it suffices to prove the lemma for $i=2$. In this case the lemma follows from the isomorphism

$$
K_{2}\left(k[\varepsilon]_{n}\right) \simeq K_{2}(k) \oplus \frac{\Omega_{k[\varepsilon]_{n}}^{1}}{\Omega_{k}^{1}+d\left(k[\varepsilon]_{n}\right)},
$$

[Graham 1973, Theorem 3] and the observation that $k^{\times} \otimes k[\varepsilon]_{n}^{\times}$surjects onto the expression on the right, under this isomorphism. Note that $K_{2}^{M}\left(k[\varepsilon]_{n}\right)=K_{2}\left(k[\varepsilon]_{n}\right)$ [Guin 1989].

Proposition 6.1.2. The inclusion $\gamma_{k[\varepsilon]_{n}}(2)_{\mathbb{Q}}^{\prime} \rightarrow \gamma_{k[\varepsilon]_{n}}(2)_{\mathbb{Q}}^{\circ}$ is a quasiisomorphism.

Proof. The only thing that needs justification is the surjectivity of the induced map on the degree 2 cohomology groups or equivalently the surjectivity of the composition

$$
k_{\mathbb{Q}}^{\times} \otimes_{\mathbb{Q}} V_{n}^{\circ} \rightarrow\left(\bigwedge^{2} V_{n}\right)^{\circ} \rightarrow \Omega_{k[\varepsilon]_{n}}^{1} /\left(\Omega_{k}^{1}+d\left(k[\varepsilon]_{n}\right)\right),
$$

where the last map is the one in the proof of Proposition 5.1.4. But this is exactly Lemma 6.1.1. 
6.2. The construction of Bloch and Esnault with higher modulus. For the rest of the section we assume that $k$ is algebraically closed. In [2003], Bloch and Esnault construct the additive weight 2 complex with modulus 2 ; their proof goes through to give a construction for all moduli $n \geq 2$. We describe the properties of this complex below. The proofs and the details of the construction can be found in [Bloch and Esnault 2003, Section 2].

Following their notation, we let $R$ be the local ring of 0 in $\mathbb{A}_{k}^{1}$. The localization (away from 0 ) sequence for the pair $\left(k[t],\left(t^{n}\right)\right)$ splits into the exact sequences

$$
K_{2}\left(k[t],\left(t^{n}\right)\right) \rightarrow K_{2}\left(R,\left(t^{n}\right)\right) \stackrel{\partial}{\longrightarrow} \bigoplus_{x \in k^{\times}} K_{1}(k) \rightarrow K_{1}\left(k[t],\left(t^{n}\right)\right) \rightarrow 0
$$

and

$$
0 \rightarrow K_{1}\left(R,\left(t^{n}\right)\right) \stackrel{\partial}{\longrightarrow} \bigoplus_{x \in k^{\times}} K_{0}(k) \rightarrow K_{0}\left(k[t],\left(t^{n}\right)\right) \rightarrow 0,
$$

since $K_{0}\left(R,\left(t^{n}\right)\right)=0$ and the map $K_{1}\left(R,\left(t^{n}\right)\right) \rightarrow \bigoplus_{x \in k^{\times}} K_{0}(k)$ is injective, as $K_{1}\left(R,\left(t^{n}\right)\right)=1+\left(t^{n}\right)$ and the map is given by the divisor of the function [Lichtenbaum 1987, Appendix]. This description also gives a canonical identification

$$
K_{0}\left(k[t],\left(t^{n}\right)\right)=\left(k[t]_{n}^{\times}\right)^{\circ} .
$$

Using the product structure on K-theory, let

$$
T_{n} B_{2}(k):=\left(K_{2}\left(R,\left(t^{n}\right)\right) / \operatorname{im}\left(K_{1}(k) \cdot K_{1}\left(R,\left(t^{n}\right)\right)\right)_{\mathbb{Q}},\right.
$$

and let $T_{n} H_{M}^{1}(k, 2)$ be the image of $K_{2}\left(k[t],\left(t^{n}\right)\right)_{\mathbb{Q}}$ in $T_{n} B_{2}(k)$. Then the above exact sequences give the exact sequence

$$
0 \rightarrow T_{n} H_{M}^{1}(k, 2) \rightarrow T_{n} B_{2}(k) \rightarrow k^{\times} \otimes V_{n}^{\circ} \rightarrow K_{1}\left(k[t],\left(t^{n}\right)\right)_{\mathbb{Q}} \rightarrow 0 .
$$

We let $T_{n} \mathbb{Q}(2)(k): T_{n} B_{2}(k) \rightarrow k^{\times} \otimes V_{n}^{\circ}$ denote the middle part of this sequence. This is the exact generalization to higher moduli of the complex considered by Bloch and Esnault [2003] (the complex described in Section 1.2).

We will try to express the cohomology groups of $T_{n} \mathbb{Q}(2)(k)$ in terms of the groups $K_{*}\left(k[t]_{n},(t)\right)_{\mathbb{Q}}$.

First note that the long exact sequence for the pair $\left(k[t],\left(t^{n}\right)\right)$, together with the homotopy invariance of K-theory, gives canonical isomorphisms

$$
K_{*+1}\left(k[t]_{n},(t)\right) \simeq K_{*}\left(k[t],\left(t^{n}\right)\right),
$$

and therefore there is a surjection

$$
\left(\frac{K_{3}\left(k[t]_{n},(t)\right)}{K_{1}(k) \cdot K_{2}\left(k[t]_{n},(t)\right)}\right)_{\mathbb{Q}} \simeq\left(\frac{K_{2}(k[t],(t))}{K_{1}(k) \cdot K_{1}(k[t],(t))}\right)_{\mathbb{Q}} \rightarrow T_{n} H_{M}^{1}(k, 2) .
$$

Lemma 6.2.1. There is a canonical surjection $K_{3}\left(k[t]_{n},(t)\right)_{\mathbb{Q}}^{(2)} \rightarrow T_{n} H_{M}^{1}(k, 2)$. 
Proof. By [Lichtenbaum 1987, page 191],

$$
K_{3}\left(k[t]_{n}\right)_{\mathbb{Q}}=K_{3}\left(k[t]_{n}\right)_{\mathbb{Q}}^{(2)} \oplus K_{3}^{M}\left(k[t]_{n}\right)_{\mathbb{Q}},
$$

and by Lemma 6.1.1, the image of $K_{1}(k) \otimes K_{2}\left(k[t]_{n}\right)$ in $K_{3}\left(k[t]_{n}\right)$ is $K_{3}^{M}\left(k[t]_{n}\right)$. Hence that (6.2.2) is a surjection proves the lemma.

Let

$$
\rho: T_{n} B_{2}(k)=\left(\frac{K_{2}\left(R,\left(t^{n}\right)\right)}{K_{1}(k) \cdot K_{1}\left(R,\left(t^{n}\right)\right)}\right)_{\mathbb{Q}} \rightarrow\left(\frac{K_{2}\left(k[t]_{2 n-1},\left(t^{n}\right)\right)}{K_{1}(k) \cdot K_{1}\left(k[t]_{2 n-1},\left(t^{n}\right)\right)}\right)_{\mathbb{Q}}=: N
$$

denote the map induced by reduction modulo $\left(t^{2 n-1}\right)$. We will prove that $\rho$ behaves like an additive dilogarithm in this setting.

Proposition 6.2.2. The composition $K_{3}\left(k[t]_{n},(t)\right)_{\mathbb{Q}}^{(2)} \rightarrow T_{n} H_{M}^{1}(k, 2) \rightarrow N$ induced by the inclusion

$$
K_{3}\left(k[t]_{n},(t)\right)_{\mathbb{Q}}^{(2)} \rightarrow K_{3}\left(k[t]_{n},(t)\right)_{\mathbb{Q}},
$$

(6.2.2), and $\rho$ is an isomorphism.

Proof. This map is induced by the long exact sequence of the pair $\left(k[t]_{2 n-1},\left(t^{n}\right)\right)$ :

$$
\cdots \rightarrow K_{3}\left(k[t]_{n},(t)\right) \rightarrow K_{2}\left(k[t]_{2 n-1},\left(t^{n}\right)\right) \rightarrow K_{2}\left(k[t]_{2 n-1},(t)\right) \rightarrow \cdots .
$$

By Goodwillie's theorem, Remark 3.1.2 and Section 4.1.1, the map

$$
K_{3}\left(k[t]_{2 n-1},(t)\right)_{\mathbb{Q}}^{(2)} \rightarrow K_{3}\left(k[t]_{n},(t)\right)_{\mathbb{Q}}^{(2)}
$$

is equivalent to a map $k^{\oplus(2 n-2)} \rightarrow k^{\oplus(n-1)}$, where the $k^{\times}$-weights in the source range in $[2 n, 4 n-3]$, whereas in the target they range in $[n, 2 n-1]$. Therefore this last map is zero and hence $K_{3}\left(k[t]_{n},(t)\right)_{\mathbb{Q}}^{(2)} \rightarrow K_{2}\left(k[t]_{2 n-1},\left(t^{n}\right)\right)_{\mathbb{Q}}$ is injective.

By [Stienstra 1981, Theorem 1.11], $K_{2}\left(k[t]_{2 n-1},\left(t^{n}\right)\right)_{\mathbb{Q}} \simeq k^{\oplus(n-1)} \oplus\left(\Omega_{k}^{1}\right)^{\oplus(n-1)}$, and $K_{1}(k) \otimes K_{1}\left(k[t]_{2 n-1},\left(t^{n}\right)\right) \rightarrow K_{2}\left(k[t]_{2 n-1},\left(t^{n}\right)\right)_{\mathbb{Q}}$ has image $\left(\Omega_{k}^{1}\right)^{\oplus(n-1)}$.

Corollary 6.2.3. There are canonical isomorphisms

$$
\begin{aligned}
& H^{1}\left(T_{n} \mathbb{Q}(2)(k)\right) \simeq K_{3}\left(k[t]_{n},(t)\right)_{\mathbb{Q}}^{(2)} \simeq \mathrm{HC}_{2}\left(k[t]_{n},(t)\right)^{(1)}, \\
& H^{2}\left(T_{n} \mathbb{Q}(2)(k)\right) \simeq K_{2}\left(k[t]_{n},(t)\right)_{\mathbb{Q}} \simeq \mathrm{HC}_{1}\left(k[t]_{n},(t)\right) .
\end{aligned}
$$

Proof. The first isomorphism is an immediate consequence of Lemma 6.2.1 and Proposition 6.2.2, and the second is a consequence of the isomorphism

$$
K_{2}\left(k[t]_{n},(t)\right) \simeq K_{1}\left(k[t],\left(t^{n}\right)\right),
$$

which follows from the long exact sequence for $\left(k[t],\left(t^{n}\right)\right)$ and the homotopy invariance of $K$-theory. 
Proof of Corollary 1.4.1. First we note that the degree 2 terms of $T_{n} \mathbb{Q}(2)(k)$ and $\gamma_{k[\varepsilon]_{n}}(2)_{\mathbb{Q}}^{\prime}$ are both equal to $k^{\times} \otimes V_{n}^{\circ}$ and that the cohomology groups of the two complexes are canonically isomorphic (Theorem 1.3.1, Proposition 6.2.2, and Corollary 6.2.3). In both cases the projection from $k^{\times} \otimes V_{n}^{\circ}$ to the degree 2 cohomology is induced by the composition

$$
k^{\times} \otimes V_{n}^{\circ} \rightarrow K_{2}^{M}\left(k[\varepsilon]_{n}\right) \rightarrow \Omega_{k[\varepsilon]_{n}}^{1} /\left(\Omega_{k}^{1}+d\left(k[\varepsilon]_{n}\right)\right)
$$

(see the proof of Lemma 6.1.1). Therefore the images of $T_{n} B_{2}(k)$ and of $B_{2}\left(k[\varepsilon]_{n}\right)_{\mathbb{Q}}^{\prime}$ in $k^{\times} \otimes V_{n}^{\circ}$ are the same. The exact sequence (6.2.1) and Proposition 6.2.2 give a splitting of $T_{n} B_{2}(k)$; and Theorems 1.3.1 and 1.3.2 give a splitting of $B_{2}\left(k[\varepsilon]_{n}\right)_{\mathbb{Q}}^{\prime}$. This proves the corollary.

We would like to emphasize that the isomorphism given in the statement of the corollary uses the additive dilogarithm in both constructions and thus should not be considered as natural.

\section{Acknowledgments}

The author warmly thanks Professor S. Bloch for mathematical discussions and encouragement and l'Institut des Hautes Études Scientifiques for its support during the summer of 2004 where part of this paper was written. Finally, he thanks James Borger for his comments on the paper.

\section{References}

[Beǔlinson 1987] A. Beǔlinson, "Height pairing between algebraic cycles", pp. 1-24 in Current trends in arithmetical algebraic geometry (Arcata, CA, 1985), edited by K. A. Ribet, Contemp. Math. 67, Amer. Math. Soc., Providence, RI, 1987. MR 89g:11052 Zbl 0624.14005

[Beŭlinson et al. 1990] A. A. Beřlinson, A. B. Goncharov, V. V. Schechtman, and A. N. Varchenko, "Aomoto dilogarithms, mixed Hodge structures and motivic cohomology of pairs of triangles on the plane", pp. 135-172 in The Grothendieck Festschrift, I, edited by P. Cartier et al., Progr. Math. 86, Birkhäuser, Boston, 1990. MR 92h:19007

[Bloch 1977] S. Bloch, "Higher regulators, algebraic K-theory, and zeta functions of elliptic curves", lecture notes, University of California at Irvine, 1977.

[Bloch 1986] S. Bloch, "Algebraic cycles and higher K-theory", Adv. in Math. 61:3 (1986), 267304. MR 88f:18010 Zbl 0608.14004

[Bloch and Esnault 2003] S. Bloch and H. Esnault, “The additive dilogarithm”, Doc. Math. (extra volume) (2003), 131-155. MR 2005e:19006 Zbl 1052.11048

[Cathelineau 1990/91] J.-L. Cathelineau, " $\lambda$-structures in algebraic $K$-theory and cyclic homology", K-Theory 4:6 (1990/91), 591-606. MR 92k:19003 Zbl 0735.19005

[Deligne and Goncharov 2005] P. Deligne and A. B. Goncharov, "Groupes fondamentaux motiviques de Tate mixte”, Ann. Sci. École Norm. Sup. (4) 38:1 (2005), 1-56. MR 2006b:11066 Zbl 1084.14024 
[Goncharov 1995] A. B. Goncharov, "Geometry of configurations, polylogarithms, and motivic cohomology”, Adv. Math. 114:2 (1995), 197-318. MR 96g:19005 Zbl 0863.19004

[Goncharov 1999] A. Goncharov, "Volumes of hyperbolic manifolds and mixed Tate motives", $J$. Amer. Math. Soc. 12:2 (1999), 569-618. MR 99i:19004 Zbl 0919.11080

[Goncharov 2004] A. B. Goncharov, "Euclidean scissor congruence groups and mixed Tate motives over dual numbers", Math. Res. Lett. 11:5-6 (2004), 771-784. MR 2005m:11124 Zbl 1122.11043

[Goodwillie 1986] T. G. Goodwillie, "Relative algebraic $K$-theory and cyclic homology", Ann. of Math. (2) 124:2 (1986), 347-402. MR 88b:18008 Zbl 0627.18004

[Graham 1973] J. Graham, "Continuous symbols on fields of formal power series", pp. 474-486 in Algebraic K-theory, II: "Classical" algebraic K-theory and connections with arithmetic (Seattle, WA, 1972), Lecture Notes in Math. 342, Springer, Berlin, 1973. MR 51 \#442 Zbl 0272.18008

[Guin 1989] D. Guin, "Homologie du groupe linéaire et $K$-théorie de Milnor des anneaux", J. Algebra 123:1 (1989), 27-59. MR 90g:11051 Zbl 0669.20037

[Hesselholt 2005] L. Hesselholt, " $K$-theory of truncated polynomial algebras", pp. 71-110 in Handbook of $K$-theory, I, edited by E. M. Friedlander and D. R. Grayson, Springer, Berlin, 2005. MR 2006m:19005 Zbl 1102.19002

[Lichtenbaum 1987] S. Lichtenbaum, "The construction of weight-two arithmetic cohomology", Invent. Math. 88:1 (1987), 183-215. MR 88d:14011 Zbl 0615.14004

[Loday 1992] J.-L. Loday, Cyclic homology, Grundlehren der Mathematischen Wissenschaften 301, Springer, Berlin, 1992. MR 94a:19004 Zbl 0780.18009

[Loday and Quillen 1984] J.-L. Loday and D. Quillen, "Cyclic homology and the Lie algebra homology of matrices”, Comment. Math. Helv. 59:4 (1984), 569-591. MR 86i:17003 Zbl 0565.17006

[Mazza et al. 2006] C. Mazza, V. Voevodsky, and C. Weibel, Lecture notes on motivic cohomology, Clay Mathematics Monographs 2, American Mathematical Society, Providence, RI, 2006. MR 2007e:14035 Zbl 1115.14010

[Park 2007] J. Park, “Algebraic cycles and additive dilogarithm”, Int. Math. Res. Not. 2007:18 (2007), Art. ID rnm067. MR 2008i:14015 Zbl 1124.14013

[Rülling 2007] K. Rülling, "The generalized de Rham-Witt complex over a field is a complex of zero-cycles”, J. Algebraic Geom. 16:1 (2007), 109-169. MR 2007j:14006 Zbl 1122.14006

[Stienstra 1981] J. Stienstra, "On $K_{2}$ and $K_{3}$ of truncated polynomial rings", pp. 409-455 in Algebraic $K$-theory (Evanston, IL, 1980), edited by E. M. Friedlander and M. R. Stein, Lecture Notes in Math. 854, Springer, Berlin, 1981. MR 82k:13016 Zbl 0463.13007

[Suslin 1984] A. A. Suslin, "Homology of $\mathrm{GL}_{n}$, characteristic classes and Milnor $K$-theory", Trudy Mat. Inst. Steklov. 165 (1984), 188-204. In Russian; translated in Proc. Steklov Inst. Math. 165 (1985), 207-226. MR 86f:11090b Zbl 0591.18006

[Suslin 1990] A. A. Suslin, “ $K_{3}$ of a field, and the Bloch group", Trudy Mat. Inst. Steklov. 183 (1990), 180-199, 229. In Russian; translated in Proc. Steklov Inst. Math. 4 (1991), 217-239. MR 91k:19003 Zbl 0741.19005

Communicated by Hélène Esnault

Received 2007-09-14 Revised 2008-11-01 Accepted 2008-11-11

sunver@ku.edu.tr

Koc University, Department of Mathematics, Rumelifeneri Yolu, 34450 Sarlyer-Istanbul, Turkey 


\title{
Nichols algebras with standard braiding
}

\author{
Iván Ezequiel Angiono
}

\begin{abstract}
The class of standard braided vector spaces, introduced by Andruskiewitsch and the author in 2007 to understand the proof of a theorem of Heckenberger, is slightly more general than the class of braided vector spaces of Cartan type. In the present paper, we classify standard braided vector spaces with finitedimensional Nichols algebra. For any such braided vector space, we give a PBW basis, a closed formula of the dimension and a presentation by generators and relations of the associated Nichols algebra.
\end{abstract}

Introduction

1. PBW bases 38

2. Transformations of braided graded Hopf algebras 44

3. Standard braidings 52

4. Nichols algebras of standard braided vector spaces 60

5. Presentation by generators and relations of Nichols algebras of $\begin{array}{ll}\text { standard braided vector spaces } & 71\end{array}$

$\begin{array}{ll}\text { Acknowledgments } & 104\end{array}$

$\begin{array}{ll}\text { References } & 104\end{array}$

\section{Introduction}

A breakthrough in the development of the theory of Hopf algebras occurred with the discovery of quantized enveloping algebras [Drinfel'd 1987; Jimbo 1985]. This special class of Hopf algebras has been intensively studied by many authors and from many points of view. In particular, finite-dimensional analogues of quantized enveloping algebras were introduced and investigated by Lusztig [1990a; 1990b].

About ten year ago, a classification program of pointed Hopf algebras was launched by Andruskiewitsch and Schneider [1998] (see also [Andruskiewitsch and Schneider 2002b]). The success of this program depends on finding solutions to several questions, among them:

MSC2000: primary 17B37; secondary 16W20, 16W30.

Keywords: quantized enveloping algebras, Nichols algebras, automorphisms of noncommutative algebras, pointed Hopf algebras. 
Question 1 [Andruskiewitsch 2002, Question 5.9]. Given a braided vector space of diagonal type $V$, such that the entries of its matrix are roots of unity, compute the dimension of the associated Nichols algebra $\mathfrak{B}(V)$. If it is finite, give a nice presentation of $\mathfrak{B}(V)$.

Partial answers to this question were given in [Andruskiewitsch and Schneider 2000; Heckenberger 2006b] for the class of braided vector spaces of Cartan type. These answers were already crucial to proving a classification theorem for finitedimensional Hopf algebras whose group is abelian with prime divisors of the order great than 7 [Andruskiewitsch and Schneider 2005]. Later, a complete answer to the first part of Question 1 was given in [Heckenberger 2006a].

The notion of a standard braided vector space, a special kind of diagonal braided vector space, was introduced in [Andruskiewitsch and Angiono 2008], and is reviewed in Definition 3.5 below. This class includes properly the class of braided vector spaces of Cartan type.

The purpose of this paper is to develop from scratch the theory of standard braided vector spaces. Here are our main contributions:

- We give a complete classification of standard braided vector spaces with finitedimensional Nichols algebras. As usual, we may assume the connectedness of the corresponding braiding. It turns out that standard braided vector spaces are of Cartan type when the associated Cartan matrix is of type $C, D, E$ or $F$, see Proposition 3.8. For types $A, B, G$ there are standard braided vector spaces not of Cartan type; these are listed in Propositions 3.9, 3.10 and 3.11. Those of type $A_{2}$ and $B_{2}$ appeared already in [Graña 2000]. Our classification does not rely on [Heckenberger 2006a], but we can identify our examples in the tables of that reference.

- We describe a concrete PBW (Poincaré-Birkhoff-Witt) basis of the Nichols algebra of a standard braided vector space as in the previous point; this follows from the general theory of Kharchenko [1999] together with [Heckenberger 2006b, Theorem 1]. As an application, we give closed formulas for the dimension of these Nichols algebras.

- We present a concrete set of defining relations of the Nichols algebras of standard braided vector spaces as in the previous points. This is an answer to the second part of Question 1 in the standard case. We note that this seems to be new even for Cartan type, for some values of the roots of unity appearing in the picture. Essentially, these relations are either quantum Serre relations or powers of root vectors; but in some cases, there are some substitutes of the quantum Serre relations due to the smallness of the intervening root vectors. Some of these substitutes can be recognized already in the relations in [Andruskiewitsch and Dăscălescu 2005]. 
Here is the plan of this article. We start by collecting necessary tools. Namely, we recall the definition of Lyndon words and give some properties about them, such as the Shirshov decomposition, in Section 1A. Next, in Section 1B, we discuss the notions of hyperletter and hyperword, following [Kharchenko 1999] (where they are called superletter and superword); these are certain iterations of braided commutators applied to Lyndon words. In Section 1C, a PBW basis is given for any quotient of the tensor algebra of a diagonal braided vector space $V$ by a Hopf ideal using these hyperwords. This applies in particular to Nichols algebras.

In Section 2, after some technical preparations, we present a transformation of a braided graded Hopf algebra into another, with different space of degree one. This generalizes an analogous transformation for Nichols algebras given in [Heckenberger 2006b, Proposition 1]; see Section 2C.

In Section 3 we classify standard braided vector spaces with finite-dimensional Nichols algebra. In Section 3A, we prove that if the set of PBW generators is finite, the associated generalized Cartan matrix is of finite type. So in Section 3B we obtain all the standard braidings associated to Nichols algebras of finite dimension.

Section 4 is devoted to PBW bases of Nichols algebras of standard braided vector spaces with finite Cartan matrix. In Section 4A we prove that there is exactly one PBW generator whose degree corresponds to each positive root associated to the finite Cartan matrix. We give a set of PBW generators in Section 4B, following a nice presentation from [Lalonde and Ram 1995]. As a consequence, we compute the dimension in Section 4C.

The main result of this paper is the explicit presentation by generators and relations of Nichols algebras of standard braided vector spaces with finite Cartan matrix, given in Section 5. It relies on the explicit PBW basis and transformation described in Section 2C. Section 5A states some relations for Nichols algebras of standard braidings and proves facts about the coproduct. Sections 5B-5D contain the explicit presentation for types $A_{\theta}, B_{\theta}$ and $G_{2}$, respectively. For this, we establish relations among the elements of the PBW basis, inspired in [Andruskiewitsch and Dăscălescu 2005] and [Graña 2000]. We finally prove the presentation in the case of Cartan type in Section 5E. To our knowledge, this is the first self-contained exposition of Nichols algebras of braided vector spaces of Cartan type.

Notation. We fix an algebraically closed field $k$ of characteristic 0 ; all vector spaces, Hopf algebras and tensor products are considered over $k$.

For each $N>0, \mathbb{G}_{N}$ denotes the set of primitive $N$-th roots of unity in k.

Given $n \in \mathbb{N}$ and $q \in \mathrm{k}, q \notin \bigcup_{0 \leq j \leq n} \mathbb{G}_{j}$, we define

$$
\left(\begin{array}{c}
n \\
j
\end{array}\right)_{q}=\frac{(n)_{q} !}{(k)_{q} !(n-k)_{q} !}, \quad \text { where }(n)_{q} !=\prod_{j=1}^{n}(k)_{q}, \quad \text { and }(k)_{q}=\sum_{j=0}^{k-1} q^{j} \text {. }
$$


We define

$$
\mathfrak{q}_{h}(t):=\frac{t^{h}-1}{t-1} \in \mathrm{k}[t], \quad h \in \mathbb{N} ; \quad \mathfrak{q}_{\infty}(t):=\frac{1}{1-t}=\sum_{s=0}^{\infty} t^{s} \in \mathrm{k} \llbracket t \rrbracket .
$$

For each $\theta \in \mathbb{N}$ and each $n=\left(n_{1}, \ldots, n_{\theta}\right) \in \mathbb{Z}^{\theta}$, we set $x^{n}=x_{1}^{n_{1}} \cdots x_{\theta}^{n_{\theta}} \in$ $\mathrm{k} \llbracket x_{1}^{ \pm 1}, \ldots, x_{\theta}^{ \pm 1} \rrbracket$. For each $\mathbb{Z}^{\theta}$-graded vector spaces $\mathfrak{B}$, we denote by $\mathscr{H}_{\mathfrak{B}}=$ $\sum_{n \in \mathbb{Z}^{\theta}} \operatorname{dim} \mathfrak{B}^{n} x^{n}$ the Hilbert series associated to $\mathfrak{B}$.

Let $C=\bigoplus_{n \in \mathbb{N}_{0}} C_{i+j}$ be a $\mathbb{N}_{0}$-graded coalgebra, with projections $\pi_{n}: C \rightarrow C_{n}$. Given $i, j \geq 0$, we denote by

$$
\Delta_{i, j}:=\left(\pi_{i} \otimes \pi_{j}\right) \circ \Delta: C_{i+j} \rightarrow C_{i} \otimes C_{j},
$$

the $(i, j)$-th component of the comultiplication.

\section{PBW bases}

Let $A$ be an algebra, $P, S \subset A$ and $h: S \mapsto \mathbb{N} \cup\{\infty\}$. Let also $<$ be a linear order on $S$. Let us denote by $B(P, S,<, h)$ the set

$$
\left\{p s_{1}^{e_{1}} \ldots s_{t}^{e_{t}}: t \in \mathbb{N}_{0}, s_{1}>\cdots>s_{t}, s_{i} \in S, 0<e_{i}<h\left(s_{i}\right), p \in P\right\} .
$$

If $B(P, S,<, h)$ is a linear basis of $A$, then we say that $(P, S,<, h)$ is a set of $P B W$ generators with height $h$, and that $B(P, S,<, h)$ is a $P B W$ basis of $A$. Occasionally, we shall simply say that $S$ is a PBW basis of $A$.

In this section, following [Kharchenko 1999], we describe an appropriate PBW basis of a braided graded Hopf algebra $\mathfrak{B}=\bigoplus_{n \in \mathbb{N}} \mathfrak{B}^{n}$ such that $\mathfrak{B}^{1} \cong V$, where $V$ is a braided vector space of diagonal type. This applies in particular, to the Nichols algebra $\mathfrak{B}(V)$. In Section 1A we recall the classical construction of Lyndon words. Let $V$ be a vector space together with a fixed basis. Then there is a basis of the tensor algebra $T(V)$ by certain words satisfying a special condition, called Lyndon words. Each Lyndon word has a canonical decomposition as a product of a pair of smaller Lyndon words, called the Shirshov decomposition.

We briefly recall the notions of a braided vector space $(V, c)$ of diagonal type and of a Nichols algebra in Section 1B. In Section 1C we recall the definition of the hyperletter $[l]_{c}$, for any Lyndon word $l$; this is the braided commutator of the hyperletters corresponding to the words in the Shirshov decomposition. Hyperletters are a set of generators for a PBW basis of $T(V)$ and their classes form a PBW basis of $\mathfrak{B}$.

1A. Lyndon words. Let $\theta \in \mathbb{N}$. Let $X$ be a set with $\theta$ elements and fix an enumeration $x_{1}, \ldots, x_{\theta}$ of $X$; this induces a total order on $X$. Let $\mathbb{X}$ be the corresponding vocabulary (the set of words with letters in $X$ ) and consider the lexicographical order on $\mathbb{X}$. 
Definition 1.1. An element $u \in \mathbb{X}, u \neq 1$, is called a Lyndon word if $u$ is smaller than any of its proper ends; that is, if $u=v w, v, w \in \mathbb{X}-\{1\}$, then $u<w$. The set of Lyndon words is denoted by $L$.

We shall need the following properties of Lyndon words.

(1) Let $u \in \mathbb{X}-X$. Then $u$ is Lyndon if and only if for any representation $u=u_{1} u_{2}$, with $u_{1}, u_{2} \in \mathbb{X}$ not empty, one has $u_{1} u_{2}=u<u_{2} u_{1}$.

(2) Any Lyndon word begins by its smallest letter.

(3) If $u_{1}, u_{2} \in L, u_{1}<u_{2}$, then $u_{1} u_{2} \in L$.

The basic Theorem about Lyndon words, due to Lyndon, says that any word $u \in \mathbb{X}$ has a unique decomposition

$$
u=l_{1} l_{2} \ldots l_{r},
$$

with $l_{i} \in L, l_{r} \leq \cdots \leq l_{1}$, as a product of nonincreasing Lyndon words. This is called the Lyndon decomposition of $u \in \mathbb{X}$; the $l_{i} \in L$ appearing in the decomposition (1-1) are called the Lyndon letters of $u$.

The lexicographical order of $\mathbb{X}$ turns out to be the same as the lexicographical order in the Lyndon letters. Namely, if $v=l_{1} \ldots l_{r}$ is the Lyndon decomposition of $v$, then $u<v$ if and only if

(i) the Lyndon decomposition of $u$ is $u=l_{1} \ldots l_{i}$, for some $1 \leq i<r$, or

(ii) the Lyndon decomposition of $u$ is $u=l_{1} \ldots l_{i-1} l l_{i+1}^{\prime} \ldots l_{s}^{\prime}$, for some $1 \leq i<r$, $s \in \mathbb{N}$ and $l, l_{i+1}^{\prime}, \ldots, l_{s}^{\prime}$ in $L$, with $l<l_{i}$.

Here is another useful characterization of Lyndon words.

Lemma 1.2 [Kharchenko 1999, p. 6]. Let $u \in \mathbb{X}-X$. Then $u \in L$ if and only if there exist $u_{1}, u_{2} \in L$ with $u_{1}<u_{2}$ such that $u=u_{1} u_{2}$.

Definition 1.3. Let $u \in L-X$. A decomposition $u=u_{1} u_{2}$, with $u_{1}, u_{2} \in L$ such that $u_{2}$ is the smallest end among those proper nonempty ends of $u$ is called the Shirshov decomposition of $u$.

Let $u, v, w \in L$ be such that $u=v w$. Then $u=v w$ is the Shirshov decomposition of $u$ if and only if either $v \in X$, or else if $v=v_{1} v_{2}$ is the Shirshov decomposition of $v$, then $w \leq v_{2}$.

1B. Braided vector spaces of diagonal type and Nichols algebras. A braided vector space is a pair $(V, c)$, where $V$ is a vector space and $c \in \operatorname{Aut}(V \otimes V)$ is a solution of the braid equation

$$
(c \otimes \mathrm{id})(\mathrm{id} \otimes c)(c \otimes \mathrm{id})=(\mathrm{id} \otimes c)(c \otimes \mathrm{id})(\mathrm{id} \otimes c) .
$$


We extend the braiding to $c: T(V) \otimes T(V) \rightarrow T(V) \otimes T(V)$ in the usual way. If $x, y \in T(V)$, the braided commutator is

$$
[x, y]_{c}:=\text { multiplication } \circ(\mathrm{id}-c)(x \otimes y) .
$$

Assume that $\operatorname{dim} V<\infty$ and pick a basis $X=\left\{x_{1}, \ldots, x_{\theta}\right\}$ of $V$; we may then identify $\mathrm{kX}$ with $T(V)$. We consider the following gradings of the algebra $T(V)$ :

(i) The usual $\mathbb{N}_{0}$-grading $T(V)=\bigoplus_{n \geq 0} T^{n}(V)$. If $\ell$ denotes the length of a word in $\mathbb{X}$, then $T^{n}(V)=\bigoplus_{x \in \mathbb{X}, \ell(x)=n} \mathrm{k} x$.

(ii) Let $\mathbf{e}_{1}, \ldots, \mathbf{e}_{\theta}$ be the canonical basis of $\mathbb{Z}^{\theta}$. Then $T(V)$ is also $\mathbb{Z}^{\theta}$-graded, where the degree is determined by $\operatorname{deg} x_{i}=\mathbf{e}_{i}, 1 \leq i \leq \theta$.

A braided vector space $(V, c)$ is of diagonal type with respect to the basis $x_{1}, \ldots x_{\theta}$ if there exist $q_{i j} \in \mathrm{k}^{\times}$such that $c\left(x_{i} \otimes x_{j}\right)=q_{i j} x_{j} \otimes x_{i}, 1 \leq i, j \leq \theta$. Let $\chi: \mathbb{Z}^{\theta} \times \mathbb{Z}^{\theta} \rightarrow \mathrm{k}^{\times}$be the bilinear form determined by $\chi\left(\mathbf{e}_{i}, \mathbf{e}_{j}\right)=q_{i j}, 1 \leq i, j \leq \theta$. Then

$$
c(u \otimes v)=\chi(\operatorname{deg} u, \operatorname{deg} v) v \otimes u
$$

for any $u, v \in \mathbb{X}$, where $q_{u, v}=\chi(\operatorname{deg} u, \operatorname{deg} v) \in \mathrm{k}^{\times}$. In this case, the braided commutator satisfies a "braided" Jacobi identity as well as braided derivation properties, namely

$$
\begin{aligned}
{\left[[u, v]_{c}, w\right]_{c} } & =\left[u,[v, w]_{c}\right]_{c}-\chi(\alpha, \beta) v[u, w]_{c}+\chi(\beta, \gamma)[u, w]_{c} v, \\
{[u, v w]_{c} } & =[u, v]_{c} w+\chi(\alpha, \beta) v[u, w]_{c}, \\
{[u v, w]_{c} } & =\chi(\beta, \gamma)[u, w]_{c} v+u[v, w]_{c},
\end{aligned}
$$

for any homogeneous $u, v, w \in T(V)$, of degrees $\alpha, \beta, \gamma \in \mathbb{N}^{\theta}$, respectively.

We denote by ${ }_{H}^{H}$ yळ the category of Yetter-Drinfeld module over $H$, where $H$ is a Hopf algebra with bijective antipode. Any $V \in{ }_{H}^{H}$ yळ becomes a braided vector space [Montgomery 1993]. If $H$ is the group algebra of a finite abelian group, then any $V \in{ }_{H}^{H}$ yळ is a braided vector space of diagonal type. Indeed, $V=\bigoplus_{g \in \Gamma, \chi \in \widehat{\Gamma}} V_{g}^{\chi}$, where $V_{g}^{\chi}=V^{\chi} \cap V_{g}$ with $V_{g}=\{v \in V \mid \delta(v)=g \otimes v\}$ and $V \chi=\{v \in V \mid g \cdot v=\chi(g) v$ for all $g \in \Gamma\}$. The braiding is given by $c(x \otimes y)=\chi(g) y \otimes x$, for all $x \in V_{g}, g \in \Gamma, y \in V^{\chi}, \chi \in \widehat{\Gamma}$.

Reciprocally, any braided vector space of diagonal type can be realized as a Yetter-Drinfeld module over the group algebra of an abelian group.

If $V \in{ }_{H}^{H}$ yळ, the tensor algebra $T(V)$ admits a unique structure of graded braided Hopf algebra in ${ }_{H}^{H} y \mathscr{D}$ such that $V \subseteq \mathscr{P}(V)$. Following [Andruskiewitsch and Schneider 2002b], we consider the class $\mathfrak{S}$ of all the homogeneous two-sided ideals $I \subseteq T(V)$ such that

- $I$ is generated by homogeneous elements of degree $\geq 2$, 
- $I$ is a Yetter-Drinfeld submodule of $T(V)$, and

- $I$ is a Hopf ideal: $\Delta(I) \subset I \otimes T(V)+T(V) \otimes I$.

The Nichols algebra $\mathfrak{B}(V)$ associated to $V$ is the quotient of $T(V)$ by the maximal element $I(V)$ of $\mathfrak{S}$.

Let $(V, c)$ be a braided vector space of diagonal type, and assume that $q_{i j}=q_{j i}$ for all $i, j$. Let $\Gamma$ be the free abelian group of rank $\theta$, with basis $g_{1}, \ldots, g_{\theta}$, and define the characters $\chi_{1}, \ldots, \chi_{\theta}$ of $\Gamma$ by

$$
\chi_{j}\left(g_{i}\right)=q_{i j}, \quad 1 \leq i, j \leq \theta .
$$

Consider $V$ as a Yetter-Drinfeld module over $\mathrm{k} \Gamma$ by defining $x_{i} \in V_{g_{i}}^{\chi_{i}}$.

Proposition 1.4 [Lusztig 1993, Proposition 1.2.3; Andruskiewitsch and Schneider 2002b, Proposition 2.10]. Let $a_{1}, \ldots, a_{\theta} \in \mathrm{k}^{\times}$. There is a unique bilinear form ( | ) $: T(V) \times T(V) \rightarrow \mathrm{k}$ such that $(1 \mid 1)=1$,

$$
\begin{aligned}
& \left(x_{i} \mid x_{j}\right)=\delta_{i j} a_{i} \text { for all } i, j, \\
& \left(x \mid y y^{\prime}\right)=\left(x_{(1)} \mid y\right)\left(x_{(2)} \mid y^{\prime}\right) \text { for all } x, y, y^{\prime} \in T(V) \\
& \left(x x^{\prime} \mid y\right)=\left(x \mid y_{(1)}\right)\left(x^{\prime} \mid y_{(2)}\right) \text { for all } x, x^{\prime}, y \in T(V) .
\end{aligned}
$$

This form is symmetric and also satisfies

$$
(x \mid y)=0 \quad \text { for all } x \in T(V)_{g}, y \in T(V)_{h}, g, h \in \Gamma, g \neq h .
$$

The quotient $T(V) / I(V)$, where

$$
I(V):=\{x \in T(V):(x \mid y)=0 \text { for all } y \in T(V)\}
$$

is the radical of the form, is canonically isomorphic to the Nichols algebra of $V$. Thus, ( I ) induces a nondegenerate bilinear form on $\mathfrak{B}(V)$ denoted by the same name.

If $(V, c)$ is of diagonal type, the ideal $I(V)$ is $\mathbb{Z}^{\theta}$-homogeneous, since it is the radical of a bilinear form in which the different $\mathbb{Z}^{\theta}$-homogeneous components are orthogonal; see [Andruskiewitsch and Schneider 2004, Proposition 2.10]. Hence $\mathfrak{B}(V)$ is $\mathbb{Z}^{\theta}$-graded. The following statement, that we include for later reference, is well-known.

Lemma 1.5. Let $V$ be a braided vector space of diagonal type, and consider its Nichols algebra $\mathfrak{B}(V)$.

(a) If $q_{i i}$ is a root of unity of order $N>1$, then $x_{i}^{N}=0$.

(b) If $i \neq j$, then $\left(\operatorname{ad}_{c} x_{i}\right)^{r}\left(x_{j}\right)=0$ if and only if

$$
(r)_{q_{i i}} ! \prod_{0 \leq k \leq r-1}\left(1-q_{i i}^{k} q_{i j} q_{j i}\right)=0 .
$$


(c) If $i \neq j$ and $q_{i j} q_{j i}=q_{i i}^{r}$, for some $r \leq 0$, then $\left(\operatorname{ad}_{c} x_{i}\right)^{1-r}\left(x_{j}\right)=0$.

1C. PBW basis of a quotient of the tensor algebra by a Hopf ideal. Let $(V, c)$ be a braided vector space with a basis $X=\left\{x_{1}, \ldots, x_{\theta}\right\}$; identify $T(V)$ with kX. There is an important graded endomorphism []$_{c}$ of $\mathrm{k} \mathbb{X}$ given by

$$
[u]_{c}:= \begin{cases}u & \begin{array}{ll}
u & \text { if } u=1 \text { or } u \in X \\
{\left[[v]_{c},[w]_{c}\right]_{c}} & \text { if } u \in L, \ell(u)>1 \\
{\left[u_{1}\right]_{c} \ldots\left[u_{t}\right]_{c}} & \text { if } u \in \mathbb{X}-L \text { with Lyndon decomposition } u=u_{1} \ldots u_{t} .
\end{array} \text { and } u=v w \text { is the Shirshov decomposition; }\end{cases}
$$

Now assume that $(V, c)$ is of diagonal type with respect to the basis $x_{1}, \ldots, x_{\theta}$, with matrix $\left(q_{i j}\right)$.

Definition 1.6. The hyperletter corresponding to $l \in L$ is the element $[l]_{c}$. A hyperword is a word in hyperletters, and a monotone hyperword is a hyperword of the form $W=\left[u_{1}\right]_{c}^{k_{1}} \ldots\left[u_{m}\right]_{c}^{k_{m}}$, where $u_{1}>\cdots>u_{m}$.

Remark 1.7. If $u \in L$, then $[u]_{c}$ is a homogeneous polynomial with coefficients in $\mathbb{Z}\left[q_{i j}\right]$ and $[u]_{c} \in u+\mathrm{k}_{>u}^{\ell(u)}$.

The hyperletters inherit the order from the Lyndon words; this induces in turn an ordering in the hyperwords (the lexicographical order on the hyperletters). Now, given monotone hyperwords $W, V$, it can be shown that

$$
W=\left[w_{1}\right]_{c} \ldots\left[w_{m}\right]_{c}>V=\left[v_{1}\right]_{c} \ldots\left[v_{t}\right]_{c},
$$

where $w_{1} \geq \cdots \geq w_{r}, \quad v_{1} \geq \cdots \geq v_{s}$, if and only if

$$
w=w_{1} \ldots w_{m}>v=v_{1} \ldots v_{t} .
$$

Furthermore, the principal word of the polynomial $W$, when decomposed as sum of monomials, is $w$ with coefficient 1 .

Theorem 1.8 [Rosso 1999]. Let $m, n \in L$, with $m<n$. Then the braided commutator $\left[[m]_{c},[n]_{c}\right]_{c}$ is a $\mathbb{Z}\left[q_{i j}\right]$-linear combination of monotone hyperwords $\left[l_{1}\right]_{c}, \ldots$, $\left[l_{r}\right]_{c}, l_{i} \in L$, such that

- the hyperletters of those hyperwords satisfy $n>l_{i} \geq m n$,

- $[\mathrm{mn}]_{c}$ appears in the expansion with a nonzero coefficient, and

- any hyperword appearing in this decomposition satisfies

$$
\operatorname{deg}\left(l_{1} \ldots l_{r}\right)=\operatorname{deg}(m n) .
$$

A crucial result of Rosso describes the behavior of the coproduct of $T(V)$ in the basis of hyperwords. 
Lemma 1.9 [Rosso 1999]. Let $u \in \mathbb{X}$, and $u=u_{1} \ldots u_{r} v^{m}, v, u_{i} \in L, v<u_{r} \leq$ $\cdots \leq u_{1}$ the Lyndon decomposition of $u$. Then

$$
\begin{aligned}
\Delta\left([u]_{c}\right)=1 \otimes[u]_{c}+\sum_{i=0}^{m}\left(\begin{array}{c}
m \\
i
\end{array}\right)_{q_{v, v}}\left[u_{1}\right]_{c} \ldots\left[u_{r}\right]_{c}[v]_{c}^{i} \otimes[v]_{c}^{m-i} & +\sum_{\substack{l_{1} \geq \cdots \geq l_{p}>v, l_{i} \in L \\
0 \leq j \leq m}} x_{l_{1}, \ldots, l_{p}}^{(j)} \otimes\left[l_{1}\right]_{c} \ldots\left[l_{p}\right]_{c}[v]_{c}^{j},
\end{aligned}
$$

where each $x_{l_{1}, \ldots, l_{p}}^{(j)}$ is $\mathbb{Z}^{\theta}$-homogeneous and

$$
\operatorname{deg}\left(x_{l_{1}, \ldots, l_{p}}^{(j)}\right)+\operatorname{deg}\left(l_{1} \ldots l_{p} v^{j}\right)=\operatorname{deg}(u) .
$$

As in [Ufer 2004], we consider another order in $\mathbb{X}$; it is implicit in [Kharchenko 1999].

Definition 1.10. Let $u, v \in \mathbb{X}$. We say that $u \succ v$ if and only if either $\ell(u)<\ell(v)$, or else $\ell(u)=\ell(v)$ and $u>v$ (lexicographical order). This $\succ$ is a total order, called the deg-lex order.

Note that the empty word 1 is the maximal element for $\succ$. Also, this order is invariant by right and left multiplication.

Let now $I$ be a proper ideal of $T(V)$, and set $R=T(V) / I$. Let $\pi: T(V) \rightarrow R$ be the canonical projection. Consider the subset of $\mathbb{X}$ given by

$$
G_{I}:=\left\{u \in \mathbb{X}: u \notin \mathrm{k} \mathbb{X}_{\succ u}+I\right\} .
$$

(a) If $u \in G_{I}$ and $u=v w$, then $v, w \in G_{I}$.

(b) Any word $u \in G_{I}$ factorizes uniquely as a nonincreasing product of Lyndon words in $G_{I}$.

Proposition 1.11 ([Kharchenko 1999]; see also [Rosso 1999]). The set $\pi\left(G_{I}\right)$ is a basis of $R$.

In what follows, $I$ is a Hopf ideal. We seek to find a PBW basis by hyperwords of the quotient $R$ of $T(V)$. For this, we look at the set

$$
S_{I}:=G_{I} \cap L .
$$

We then define the function $h_{I}: S_{I} \rightarrow\{2,3, \ldots\} \cup\{\infty\}$ by

$$
h_{I}(u):=\min \left\{t \in \mathbb{N}: u^{t} \in \mathrm{k} \mathbb{X}_{\succ u^{t}}+I\right\} .
$$

The next result plays a fundamental role in this paper. 
Theorem 1.12 [Kharchenko 1999]. Keep the notation above. Then

$$
B_{I}^{\prime}:=B\left(\{1+I\},\left[S_{I}\right]_{c}+I,<, h_{I}\right)
$$

is a PBW basis of $H=T(V) / I$.

The next three results are consequences of Theorem 1.12; see [Kharchenko 1999] for their proofs.

Corollary 1.13. A word $u$ belongs to $G_{I}$ if and only if the corresponding hyperletter $[u]_{c}$ is not a linear combination, modulo I, of hyperwords $[w]_{c}, w \succ u$, where all the hyperwords have their hyperletters in $S_{I}$.

Proposition 1.14. In the conditions of the Theorem 1.12, if $v \in S_{I}$ is such that $h_{I}(v)<\infty$, then $q_{v, v}$ is a root of unity. In this case, if $t$ is the order of $q_{v, v}$, then $h_{I}(v)=t$.

Corollary 1.15. If $h_{I}(v):=h<\infty$, then $[v]^{h}$ is a linear combination of hyperwords $[w]_{c}, w \succ v^{h}$.

\section{Transformations of braided graded Hopf algebras}

In Section 2C, we shall introduce a transformation over certain graded braided Hopf algebras, generalizing [Heckenberger 2006b, Proposition 1]. It is an instrumental step in the proof of Theorem 5.25, one of the main results of this article.

2A. Preliminaries on braided graded Hopf algebras. Let $H$ be the group algebra of an abelian group $\Gamma$. Let $V \in{ }_{H}^{H_{\mathscr{O}}}$ y with a basis $X=\left\{x_{1}, \ldots, x_{\theta}\right\}$ such that $x_{i} \in V_{g_{i}}^{\chi_{i}}, 1 \leq i \leq \theta$. Let $q_{i j}=\chi_{j}\left(g_{i}\right)$, so that $c\left(x_{i} \otimes x_{j}\right)=q_{i j} x_{j} \otimes x_{i}, 1 \leq i, j \leq \theta$.

We fix an ideal $I$ in the class $\mathfrak{S}$; we assume that $I$ is $\mathbb{Z}^{\theta}$-homogeneous. Let $\mathfrak{B}:=T(V) / I$ : this is a braided graded Hopf algebra, $\mathfrak{B}^{0}=\mathrm{k} 1$ and $\mathfrak{B}^{1}=V$. By definition of $I(V)$, there exists a canonical epimorphism of braided graded Hopf algebras $\pi: \mathfrak{B} \rightarrow \mathfrak{B}(V)$. Let $\sigma_{i}: \mathfrak{B} \rightarrow \mathfrak{B}$ be the algebra automorphism given by the action of $g_{i}$.

For the proof of the next result, see [Andruskiewitsch and Schneider 2002b, 2.8], for example.

Proposition 2.1. (1) For each $1 \leq i \leq \theta$, there exists a uniquely determined (id, $\left.\sigma_{i}\right)$-derivation $D_{i}: \mathfrak{B} \rightarrow \mathfrak{B}$ with $D_{i}\left(x_{j}\right)=\delta_{i, j}$ for all $j$.

(2) $I=I(V)$ if and only if $\bigcap_{i=1}^{\theta} \operatorname{ker} D_{i}=\mathrm{k} 1$.

These operators are defined for each $x \in \mathfrak{B}^{k}, k \geq 1$ by the formula

$$
\Delta_{n-1,1}(x)=\sum_{i=1}^{\theta} D_{i}(x) \otimes x_{i} .
$$


Analogously, we can define operators $F_{i}: \mathfrak{B} \rightarrow \mathfrak{B}$ by $F_{i}(1)=0$ and

$$
\Delta_{1, n-1}(x)=\sum_{i=1}^{\theta} x_{i} \otimes F_{i}(x) \quad \text { for all } x \in \bigoplus_{k>0} \mathfrak{B}^{k} .
$$

Let $\chi$ be as in Section 1B. Consider the action $\triangleright$ of $k \mathbb{Z}^{\theta}$ on $\mathfrak{B}$ given by

$$
\mathbf{e}_{i} \triangleright b=\chi\left(\mathbf{u}, \mathbf{e}_{i}\right) b, \quad b \text { homogeneous of degree } \mathbf{u} \in \mathbb{Z}^{\theta} .
$$

Such operators $F_{i}$ satisfy $F_{i}\left(x_{j}\right)=\delta_{i, j}$ for all $j$, and

$$
F_{i}\left(b_{1} b_{2}\right)=F_{i}\left(b_{1}\right) b_{2}+\left(\mathbf{e}_{i} \triangleright b_{1}\right) F_{i}\left(b_{2}\right), \quad b_{1}, b_{2} \in \mathfrak{B} .
$$

Let $z_{r}^{(i j)}:=\left(\operatorname{ad}_{c} x_{i}\right)^{r}\left(x_{j}\right), i, j \in\{1, \ldots, \theta\}, i \neq j$ and $r \in \mathbb{N}_{0}$.

Remark 2.2. The operators $D_{i}, F_{i}$ satisfy

$$
\begin{aligned}
& D_{i}\left(x_{i}^{n}\right)=(n)_{q_{i i}} x_{i}^{n-1}, \\
& D_{i}\left(\left(\operatorname{ad}_{c} x_{i}\right)^{r}\left(x_{j_{1}} \ldots x_{j_{s}}\right)\right)=0 \text { for } r, s \geq 0, j_{k} \neq i, \\
& D_{j}\left(z_{r}^{(i j)}\right)=\prod_{k=0}^{r-1}\left(1-q_{i i}^{k} q_{i j} q_{j i}\right) x_{i}^{r} \text { for } r \geq 0, \\
& F_{i}\left(z_{m}^{(i j)}\right)=(m)_{q_{i i}}\left(1-q_{i i}^{m-1} q_{i j} q_{j i}\right) z_{m-1}^{(i j)}, \\
& F_{j}\left(z_{m}^{(i j)}\right)=0, m \geq 1 .
\end{aligned}
$$

The proof of the first three identities is as in [Andruskiewitsch and Schneider 2004, Lemma 3.7]; the proof of the last two is by induction on $m$.

For each pair $1 \leq i, j \leq \theta, i \neq j$, we define

$$
\begin{aligned}
M_{i, j}(\mathfrak{B}) & :=\left\{\left(\operatorname{ad}_{c} x_{i}\right)^{m}\left(x_{j}\right): m \in \mathbb{N}\right\} ; \\
m_{i j} & :=\min \left\{m \in \mathbb{N}_{0}:(m+1)_{q_{i i}}\left(1-q_{i i}^{m} q_{i j} q_{j i}\right)=0\right\} .
\end{aligned}
$$

Then either $q_{i i}^{m_{i j}} q_{i j} q_{j i}=1$, or $q_{i i}^{m_{i j}+1}=1$, if $q_{i i}^{m} q_{i j} q_{j i} \neq 1$ for all $m=0,1, \ldots, m_{i j}$, or such $m_{i j}$ does not exist, in which case we consider $m_{i j}=\infty$.

If $\mathfrak{B}=\mathfrak{B}(V)$, we write simply $M_{i, j}=M_{i, j}(\mathfrak{B}(V))$. Note that $\left(\operatorname{ad}_{c} x_{i}\right)^{m_{i j}+1} x_{j}=0$ and $\left(\operatorname{ad}_{c} x_{i}\right)^{m_{i j}} x_{j} \neq 0$, by Lemma 1.5 , so

$$
\left|M_{i, j}\right|=m_{i j}+1 \text {. }
$$

By Theorem 1.12, the braided graded Hopf algebra $\mathfrak{B}$ has a PBW basis consisting of homogeneous elements (with respect to the $\mathbb{Z}^{\theta}$-grading). As in [Heckenberger 2006b], we can even assume that

$\circledast$ The height of a PBW generator $[u], \operatorname{deg}(u)=d$, is finite if and only if $2 \leq$ $\operatorname{ord}\left(q_{u, u}\right)<\infty$, and in such case, $h_{I(V)}(u)=\operatorname{ord}\left(q_{u, u}\right)$. 
This is possible because if the height of $[u], \operatorname{deg}(u)=d$, is finite, then $2 \leq$ $\operatorname{ord}\left(q_{u, u}\right)=m<\infty$, by Proposition 1.14. And if $2 \leq \operatorname{ord}\left(q_{u, u}\right)=m<\infty$, but $h_{I(V)}(u)$ is infinite, we can add $[u]^{m}$ to the PBW basis: in this case, $h_{I(V)}(u)=$ $\operatorname{ord}\left(q_{u, u}\right)$, and $q_{u^{m}, u^{m}}=q_{u, u}^{m^{2}}=1$.

Let $\Delta^{+}(\mathfrak{B}) \subseteq \mathbb{N}^{n}$ be the set of degrees of the generators of the PBW basis, counted with their multiplicities and let also $\Delta(\mathfrak{B})=\Delta^{+}(\mathfrak{B}) \cup\left(-\Delta^{+}(\mathfrak{B})\right)$ : $\Delta^{+}(\mathfrak{B})$ is independent of the choice of the PBW basis with the property $\circledast$ (see [Andruskiewitsch and Angiono 2008, Lemma 2.18] for a proof of this statement).

In what follows, we write

$$
q_{\alpha}:=\chi(\alpha, \alpha), \quad N_{\alpha}:=\operatorname{ord} q_{\alpha}, \quad \alpha \in \Delta^{+}(\mathfrak{B}) .
$$

2B. Auxiliary results. Let $I$ be $\mathbb{Z}^{\theta}$-homogeneous ideal in $\mathfrak{S}$ and $\mathfrak{B}=T(V) / I$ as in Section 2A. We shall use repeatedly the following fact.

In what follows, we use the convention ord $1=1$.

Remark 2.3. If $x_{i}^{N}=0$ in $\mathfrak{B}$ with $N$ minimal (this is called the order of nilpotency of $\left.x_{i}\right)$, then $q_{i i}$ is a root of 1 of order $N$. Hence $\left(\operatorname{ad}_{c} x_{i}\right)^{N} x_{j}=0$.

The following result extends (18) in the proof of [Heckenberger 2006b, Proposition 1].

Lemma 2.4. For $i \in\{1, \ldots, \theta\}$, let $\mathscr{K}_{i}$ be the subalgebra generated by $\bigcup_{j \neq i} M_{i, j}(\mathfrak{B})$ and denote by $n_{i}$ the order of $q_{i i}$. Then there are isomorphisms of graded vector spaces

- $\operatorname{ker}\left(D_{i}\right) \cong \mathscr{K}_{i} \otimes \mathrm{k}\left[x_{i}^{n_{i}}\right]$, if $1<\operatorname{ord} q_{i i}<\infty$ but $x_{i}$ is not nilpotent, or

- $\operatorname{ker}\left(D_{i}\right) \cong \mathcal{K}_{i}$, if ord $q_{i i}$ is the order of nilpotency of $x_{i}$ or $q_{i i}=1$.

Moreover,

$$
\mathfrak{B} \cong \mathscr{K}_{i} \otimes \mathrm{k}\left[x_{i}\right] .
$$

Proof. We assume for simplicity $i=1$ and consider the PBW basis obtained in the Theorem 1.12. Now $x_{1} \in S_{I}$, and it is the least element of $S_{I}$, so each element of $B_{I}^{\prime}$ is of the form $\left[u_{1}\right]^{s_{1}} \ldots\left[u_{k}\right]^{s_{k}} x_{1}^{s}$, with $u_{k}<\cdots<u_{1}, u_{i} \in S_{I} \backslash\left\{x_{1}\right\}, 0<s_{i}<$ $h_{I}\left(u_{i}\right), 0 \leq s<h_{I}\left(x_{1}\right)$. Call $S^{\prime}=S_{I} \backslash\left\{x_{1}\right\}$, and

$$
B_{2}:=B\left(1+I,\left[S^{\prime}\right]_{c}+I,<,\left.h_{I}\right|_{S^{\prime}}\right),
$$

that is, the PBW set generated by $\left[S^{\prime}\right]_{c}+I$, whose height is the restriction of the height of the PBW basis corresponding to $S^{\prime}$. We have

$$
\mathfrak{B} \cong \mathrm{k} B_{2} \otimes \mathrm{k}\left[x_{1}\right] .
$$

By (2-3), any $\left(\operatorname{ad}_{c} x_{1}\right)^{r}\left(x_{j}\right) \in \operatorname{ker}\left(D_{1}\right)$; as $D_{1}$ is a skew-derivation, we have $\mathscr{K}_{1} \subseteq \operatorname{ker}\left(D_{1}\right)$. 
Also, $\operatorname{ad}_{c} x_{1}$ is a $\left(\sigma_{1}, \mathrm{id}\right)$-derivation of $\mathfrak{B}$. This derivation restricts to an endomorphism of the algebra $\mathscr{K}_{1}$, because if we apply ad $x_{c} x_{1}$ to the generators of $\mathscr{K}_{1}$, we obtain another generators (or 0 ).

We shall prove by induction on the length of $u$ that $[u]_{c} \in \mathscr{Y}_{1}$ for each $u \in L \backslash\left\{x_{1}\right\}$. If $u=x_{j}, j>1$, then $[u]_{c}=x_{j} \in \mathscr{K}_{1}$. Now let $u \in L \backslash\left\{x_{1}\right\}$ be of length greater than 1 , and $(v, w)$ its Lyndon decomposition. Then:

- If $v \neq x_{1}$, then $[v]_{c},[w]_{c} \in \mathscr{K}_{1}$ by induction hypothesis, so

$$
[u]_{c}=[v]_{c}[w]_{c}-\chi(\operatorname{deg} v, \operatorname{deg} w)[w]_{c}[v]_{c} \in \mathscr{K}_{1},
$$

because $\mathscr{K}_{1}$ is a subalgebra.

- If $v=x_{1}$, then $[u]_{c}=\operatorname{ad}_{c} x_{1}\left([w]_{c}\right) \subset \operatorname{ad}_{c} x_{1}\left(\mathscr{K}_{1}\right) \subseteq \mathscr{K}_{1}$, because by induction hypothesis $[w]_{c} \in \mathscr{K}_{1}$.

Then we prove that $[L]_{c} \backslash\left\{x_{1}\right\} \subseteq \mathscr{K}_{1}$, and $B_{2}$ is generated by $[L]_{c} \backslash\left\{x_{1}\right\}$; that is, $\mathrm{k} B_{2} \subseteq \mathscr{K}_{1}$, and $D_{1}\left(B_{2}\right)=0$.

If $u \in \operatorname{ker}\left(D_{1}\right)$, we can write $[u]_{c}=\sum_{w \in B_{I}^{\prime}} \alpha_{w}[w]_{c}$. If $w$ does not end with $x_{1}$, then $w \in B_{2}$, and $D_{1}\left([w]_{c}\right)=0$. But if $w=u_{w} x_{1}^{t_{w}},\left[u_{w}\right]_{c} \in B_{2}, 0<t_{w}<h_{I}\left(x_{1}\right)$, we have

$$
D_{1}\left([w]_{c}\right)=\left(t_{w}\right)_{q_{11}^{-1}}\left[u_{w}\right]_{c} x_{1}^{t_{w}-1}
$$

where $\left(t_{w}\right)_{q_{11}^{-1}} \neq 0$ if $n_{i}$ does not divide $t_{w}$. Then

$$
0=D_{1}\left([u]_{c}\right)=\sum_{w \in B_{I}^{\prime} / t_{w}>0} \alpha_{w}\left(t_{w}\right)_{q_{11}^{-1}}\left[u_{w}\right]_{c} x_{1}^{t_{w}-1},
$$

But $\left[u_{w}\right]_{c} x_{1}^{t_{w}-1} \in B_{2}$, and $B_{2}$ is a basis, so $\alpha_{w}=0$ for each $w$ such that $n_{i}$ does not divide $t_{w}$. Then $\operatorname{ker}\left(D_{1}\right)=\mathscr{K}_{1} \mathrm{k}\left[x_{i}^{n_{i}}\right]$, so $\operatorname{ker}\left(D_{1}\right) \simeq \mathscr{K}_{1} \otimes \mathrm{k}\left[x_{i}^{n_{i}}\right]$ as k-vector spaces. This fact and the first part conclude the proof.

2C. Transformations of certain braided graded Hopf algebras. Let $I$ be $\mathbb{Z}^{\theta}$ homogeneous ideal in $\mathfrak{S}$ and $\mathfrak{B}=T(V) / I$ as in the previous subsections. We fix $i \in\{1, \ldots, \theta\}$.

Remark 2.5. ord $q_{i i}=\min \left\{k \in \mathbb{N}: F_{i}^{k}=0\right\}$, if $q_{i i} \neq 1$.

Proof. If $k \in \mathbb{N}$, then $F_{i}\left(x_{i}^{k}\right)=(k)_{q_{i i}} x_{i}^{k-1}$, and for all $k \in \mathbb{N}$,

$$
F_{i}^{k}\left(x_{i}^{k}\right)=(k) q_{i i}^{-1} !
$$

That is, if $F_{i}^{k}=0$, then $(k)_{i i} q_{i i}^{-1}=0$. Hence ord $q_{i i} \leq \min \left\{k \in \mathbb{N}: F_{i}^{k}=0\right\}$. Reciprocally, if $q_{i i}$ is a root of unity of order $k$, then $F_{i}^{k}\left(x_{i}^{t}\right)=0$ for all $t \geq k$ by the previous claim, and $F_{i}^{k}\left(x_{i}^{t}\right)=0$ for all $t<k$ by degree arguments. Since $F_{i}\left(x_{j}\right)=0$ for $j \neq i, F_{i}^{k}=0$. 
We now extend some considerations in [Heckenberger 2006b, p. 180]. We consider the Hopf algebra defined by

$$
H_{i}:= \begin{cases}\mathrm{k}\left\langle y, e_{i}, e_{i}^{-1} \mid e_{i} y-q_{i i}^{-1} y e_{i}, y^{N_{i}}\right\rangle & \text { where } N_{i} \text { is the order of nilpotency } \\ \mathrm{k}\left\langle y, e_{i}, e_{i}^{-1} \mid e_{i} y-q_{i i}^{-1} y e_{i}\right\rangle & \text { of } x_{i} \text { in } \mathfrak{B}, \text { if } x_{i} \text { is nilpotent }\end{cases}
$$

together with $\Delta\left(e_{i}\right)=e_{i} \otimes e_{i}, \Delta(y)=e_{i} \otimes y+y \otimes 1$.

Notice that $\Delta$ is well-defined by Remark 2.3. We also consider the action $\triangleright$ of $H_{i}$ on $\mathfrak{B}$ given by

$$
e_{i} \triangleright b=\chi\left(\mathbf{u}, \mathbf{e}_{i}\right) b, \quad y \triangleright b=F_{i}(b),
$$

if $b$ is homogeneous of degree $\mathbf{u} \in \mathbb{N}^{\theta}$, extending the previous one defined in (2-1). The action is well-defined by Remark 2.3 and because

$$
\left(e_{i} y\right) \triangleright b=e_{i} \triangleright\left(F_{i}(b)\right)=q_{i i}^{-1} F_{i}\left(e_{i} \triangleright b\right)=\left(q_{i i}^{-1} y e_{i}\right) \triangleright b \quad \text { for } b \in \mathfrak{B} .
$$

It is easy to see that $\mathfrak{B}$ is an $H_{i}$-module algebra; hence we can form

$$
\mathscr{A}_{i}:=\mathfrak{B} \# H_{i} .
$$

Also, if we denote explicitly by $\cdot$ the multiplication in $\mathscr{A}_{i}$, we have

$$
(1 \# y) \cdot(b \# 1)=\left(e_{i} \triangleright b \# 1\right) \cdot(1 \# y)+F_{i}(b) \# 1 \quad \text { for all } b \in \mathfrak{B} .
$$

As in [Heckenberger 2006b], $\mathscr{A}_{i}$ is a left Yetter-Drinfeld module over $\mathrm{k} \Gamma$, where the action and the coaction are given by

$$
\begin{aligned}
& g_{k} \cdot x_{j} \# 1=q_{k j} x_{j} \# 1, \quad g_{k} \cdot 1 \# y=q_{k i}^{-1} 1 \# y, \quad g_{k} \cdot 1 \# e_{i}=1 \# e_{i}, \\
& \delta\left(x_{j} \# 1\right)=g_{j} \otimes x_{j} \# 1, \delta(1 \# y)=g_{i}^{-1} \otimes 1 \# y, \delta\left(1 \# e_{i}\right)=1 \otimes 1 \# e_{i},
\end{aligned}
$$

for each pair $k, j \in\{1, \ldots, \theta\}$. Also, $\mathscr{A}_{i}$ is a $\mathrm{k} \Gamma$-module algebra.

We now prove a generalization of [Heckenberger 2006b, Proposition 1] in the more general context of our braided Hopf algebras $\mathfrak{B}$. Although the general strategy of the proof is similar as in loc. cit., many points need slightly different argumentations here.

Theorem 2.6. Keep the notation above. Assume that $M_{i, j}(\mathfrak{B})$ is finite and

$$
\left|M_{i, j}(\mathfrak{B})\right|=m_{i j}+1, \quad j \in\{1, \ldots, \theta\}, j \neq i .
$$

(1) Let $V_{i}$ be the vector subspace of $\mathscr{A}_{i}$ generated by

$$
\left\{\left(\operatorname{ad}_{c} x_{i}\right)^{m_{i j}}\left(x_{j}\right) \# 1: j \neq i\right\} \cup\{1 \# y\} .
$$


The subalgebra $s_{i}(\mathfrak{B})$ of $\mathscr{A}_{i}$ generated by $V_{i}$ is a graded algebra such that $s_{i}(\mathfrak{B})^{1} \cong V_{i}$. There exist skew derivations $Y_{i}: s_{i}(\mathfrak{B}) \rightarrow s_{i}(\mathfrak{B})$ such that, for all $b_{1}, b_{2} \in s_{i}(\mathfrak{B})$, and $l, j \in\{1, \ldots, \theta\}, j \neq i$,

$$
\begin{aligned}
& Y_{j}\left(b_{1} b_{2}\right)=b_{1} Y_{j}\left(b_{2}\right)+Y_{j}\left(b_{2}\right)\left(g_{i}^{-m_{i j}} g_{j}^{-1} \cdot b_{2}\right), \\
& Y_{i}\left(b_{1} b_{2}\right)=b_{1} Y_{i}\left(b_{2}\right)+Y_{i}\left(b_{1}\right)\left(g_{i}^{-1} \cdot b_{1}\right), \\
& Y_{l}\left(\left(\operatorname{ad}_{c} x_{i}\right)^{m_{i j}}\left(x_{j}\right) \# 1\right)=\delta_{l j}, \quad Y_{l}(1 \# y)=\delta_{l i} .
\end{aligned}
$$

(2) Set $N_{i}:=\left\{n \in \mathbb{N}: n \mathbf{e}_{i} \in \Delta(\mathfrak{B})\right\}$ (by the previous remarks, $N_{i}=\{1\}$ or $N_{i}=$ $\left.\left\{1, h_{i}\right\}\right)$. The Hilbert series of $s_{i}(\mathfrak{B})$ satisfies

$$
\mathscr{H}_{s_{i}(\mathfrak{B})}=\left(\prod_{\alpha \in \Delta^{+}(\mathfrak{B}) \backslash N_{i} \mathbf{e}_{i}} \mathfrak{q}_{h_{\alpha}}\left(X^{s_{i}(\alpha)}\right)\right)\left(\prod_{s \in N_{i}} \mathfrak{q}_{h_{s \mathbf{s}_{i}}}\left(x_{i}^{s}\right)\right) .
$$

Therefore, if $s_{i}(\mathfrak{B})$ is a graded braided Hopf algebra,

$$
\Delta^{+}\left(s_{i}(\mathfrak{B})\right)=\left\{s_{i}\left(\Delta^{+}(\mathfrak{B})\right) \backslash-N_{i} \mathbf{e}_{i}\right\} \cup N_{i} \mathbf{e}_{i} .
$$

(3) If $\mathfrak{B}=\mathfrak{B}(V)$, the algebra $s_{i}(\mathfrak{B})$ is isomorphic to the Nichols algebra $\mathfrak{B}\left(V_{i}\right)$.

Proof. (i) Note that $V_{i}$ is a Yetter-Drinfeld submodule over $\mathrm{k} \Gamma$ of $\mathscr{A}_{i}$. Now, $\mathscr{A}_{i} \cong \mathfrak{B} \otimes H_{i}$ as graded vector spaces. Let $\mathscr{K}_{i}$ be the subalgebra generated by $\bigcup_{j \neq i} M_{i, j}(\mathfrak{B})$, as in Lemma 2.4. Then $s_{i}(\mathfrak{B}) \subseteq \mathcal{K}_{i} \otimes \mathrm{k}[y]$, since $F_{i}$ is a skewderivation and $F_{i}\left(z_{k}^{(i j)}\right)=(k)_{q_{i i}}\left(1-q_{i i}^{k-1} q_{i j} q_{j i}\right) z_{k-1}^{(i j)}$, by (2-5). From (2-10),

$$
(1 \# y) \cdot\left(z_{m_{i j}}^{(i j)} \# 1\right)=\left(z_{m_{i j}}^{(i j)} \# 1\right) \cdot(1 \# y)+F_{i}\left(z_{m_{i j}}^{(i j)}\right) \# 1 .
$$

Also, since $m_{i j}+1=\left|M_{i, j}(\mathfrak{B})\right|$, we have $\left(m_{i j}\right)_{q_{i i}}\left(1-q_{i i}^{m_{i j}-1} q_{i j} q_{j i}\right) \neq 0$, so $z_{m_{i j}-1}^{(i j)} \# 1$ lies in $s_{i}(\mathfrak{B})$, and by induction each $z_{k}^{(i j)} \# 1$, for $k=0, \ldots, m_{i j}-1$, is an element of $s_{i}(\mathfrak{B})$. Then $\mathcal{K}_{i} \otimes \mathrm{k}[y] \subseteq s_{i}(\mathfrak{B})$, and therefore

$$
s_{i}(\mathfrak{B})=\mathscr{K}_{i} \otimes \mathrm{k}[y] .
$$

Thus, $s_{i}(\mathfrak{B})$ is a graded algebra in ${ }_{\mathrm{k} \Gamma}^{\mathrm{k} \Gamma} \mathrm{y} \mathscr{D}$ with $s_{i}(\mathfrak{B})^{1}=V_{i}$. We have to find the skew derivations $Y_{l} \in \operatorname{End}\left(s_{i}(\mathfrak{B})\right), l=1, \ldots, \theta$. Set $Y_{i}:=\left.g_{i}^{-1} \circ \operatorname{ad}\left(x_{i} \# 1\right)\right|_{s_{i}(\mathfrak{B})}$. Then, for each $b \in \mathscr{K}_{i}$ and each $j \neq i$,

$$
\begin{aligned}
& \operatorname{ad}\left(x_{i} \# 1\right)(b \# 1)=\left(\operatorname{ad}_{c} x_{i}\right)(b) \# 1, \\
& \operatorname{ad}\left(x_{i} \# 1\right)\left(\left(\operatorname{ad}_{c} x_{i}\right)^{m_{i j}}\left(x_{j}\right) \# 1\right)=\left(\operatorname{ad}_{c} x_{i}\right)^{m_{i j}+1}\left(x_{j}\right) \# 1=0 .
\end{aligned}
$$

Also,

$$
\begin{aligned}
Y_{i}(1 \# y) & =g_{i}^{-1} \cdot\left(\left(x_{i} \# 1\right) \cdot(1 \# y)-\left(g_{i} \cdot(1 \# y)\right) \cdot\left(x_{i} \# 1\right)\right) \\
& =g_{i}^{-1} \cdot\left(x_{i} \# y+1-q_{i i}\left(q_{i i}^{-1} x_{i} \# y\right)\right)=1 .
\end{aligned}
$$

Thus $Y_{i} \in \operatorname{End}\left(s_{i}(\mathfrak{B})\right)$ satisfies (2-14). 
Therefore, $\operatorname{ad}\left(x_{i} \# 1\right)\left(b_{1} b_{2}\right)=\operatorname{ad}\left(x_{i} \# 1\right)\left(b_{1}\right) b_{2}+\left(g_{i} \cdot b_{1}\right) \operatorname{ad}\left(x_{i} \# 1\right)\left(b_{2}\right)$, for each pair $b_{1}, b_{2} \in s_{i}(\mathfrak{B})$, so we conclude that $\operatorname{ad}\left(x_{i} \# 1\right)\left(s_{i}(\mathfrak{B})\right) \subseteq s_{i}(\mathfrak{B})$, and $Y_{i} \in$ $\operatorname{End}\left(s_{i}(\mathfrak{B})\right)$ satisfies $(2-13)$.

Before proving that $Y_{i}$ satisfies (2-12), we need to establish some preliminary facts. Let us fix $j \neq i$, and let $z_{k}^{(i j)}=\left(\operatorname{ad}_{c} x_{i}\right)^{k}\left(x_{j}\right)$ as before. We define inductively

$$
\hat{z}_{0}^{(i j)}:=D_{j}, \quad \hat{z}_{k+1}^{(i j)}:=D_{i} \hat{z}_{k}^{(i j)}-q_{i i}^{k} q_{i j} \hat{z}_{k+1}^{(i j)} D_{i} \in \operatorname{End}(\mathfrak{B}) .
$$

We calculate

$$
\begin{aligned}
\lambda_{i j} & :=\hat{z}_{m_{i j}}^{(i j)}\left(z_{m_{i j}}^{(i j)}\right)=\sum_{s=0}^{m_{i j}} a_{s} D_{i}^{m_{i j}-s} D_{j} D_{i}^{s}\left(z_{m_{i j}}^{(i j)}\right) \\
& =\left(D_{i}\right)^{m_{i j}}\left(D_{j}\right)\left(z_{m_{i j}}^{(i j)}\right)=\alpha_{m_{i j}}\left(m_{i j}\right)_{q_{i i}} ! \in \mathrm{k}^{\times},
\end{aligned}
$$

where $a_{s}=(-1)^{k}\left(\begin{array}{c}m \\ k\end{array}\right)_{q_{i i}} q_{i i}^{k(k-1) / 2} q_{i j}^{k}$.

Note that $\left(D_{i}\right)^{m_{i j}+1} D_{j}(b)=0$ for all $b \in M_{i, k}, k \neq i, j$, and that

$$
\left(D_{i}\right)^{m_{i j}+1} D_{j}\left(z_{r}^{(i j)}\right)=\left(D_{i}\right)^{m_{i j}+1}\left(q_{j i}^{-r} \alpha_{r} x_{i}^{r}\right)=0 \quad \text { for all } r \leq m_{i j},
$$

so $\left(D_{i}\right)^{m_{i j}+1} D_{j}\left(\mathscr{K}_{i}\right)=0$. This implies that $\hat{z}_{m_{i j}}^{(i j)}(b) \in \mathscr{K}_{i}$, for each $b \in \mathscr{K}_{i}$. Now define $Y_{j} \in \operatorname{End}\left(s_{i}(\mathfrak{B})\right)$ by

$$
Y_{j}\left(b \# y^{m}\right):=q_{i i}^{m m_{i j}} q_{j i}^{m} \lambda_{i j}^{-1} \hat{z}_{m_{i j}}^{(i j)}(b) \# y^{m} \quad \text { for } b \in \mathcal{K}_{i}, m \in \mathbb{N} .
$$

We have $Y_{j}(1 \# y)=0$, and moreover $Y_{j}\left(\left(\operatorname{ad}_{c} x_{i}\right)^{m_{i l}}\left(x_{l}\right) \# 1\right)=0$ if $l \neq i, j$. By the choice of $\lambda_{i j}, Y_{j}\left(\left(\operatorname{ad}_{c} x_{i}\right)^{m_{i j}}\left(x_{j}\right) \# 1\right)=1$.

Now, using that $D_{k}\left(g_{l} \cdot b\right)=q_{k l} g_{l} \cdot\left(D_{k}(b)\right)$ for each $b \in \mathfrak{B}$ and $k, l \in\{1, \ldots, \theta\}$, we prove inductively that for $b_{1}, b_{2} \in \mathscr{K}_{i}$,

$$
\hat{z}_{k}^{(i j)}\left(b_{1} b_{2}\right)=b_{1} \hat{z}_{k}^{(i j)}\left(b_{2}\right)+\hat{z}_{k}^{(i j)}\left(b_{1}\right)\left(g_{i}^{k} g_{j} \cdot b_{2}\right) .
$$

Hence,

$$
\begin{aligned}
Y_{j}\left(b_{1} \# 1 \cdot b_{2} \# 1\right) & =Y_{j}\left(b_{1} b_{2} \# 1\right)=\lambda_{i j}^{-1} \hat{z}_{m_{i j}}\left(b_{1} b_{2}\right) \# 1 \\
& =b_{2} \# 1 \cdot Y_{j}\left(b_{2} \# 1\right)+Y_{j}\left(b_{1} \# 1\right) \cdot\left(g_{i}^{m_{i j}} g_{j} \cdot\left(b_{2} \# 1\right)\right) .
\end{aligned}
$$

By induction on the degree we prove that $F_{i}$ commutes with $D_{i}, D_{j}$, so

$$
\hat{z}_{m_{i j}}^{(i j)}\left(F_{i}(b)\right)=F_{i}\left(\hat{z}_{m_{i j}}^{(i j)}(b)\right) \quad \text { for all } b \in \mathfrak{B} .
$$

Consider $b \in \mathscr{K}_{i} \subseteq \operatorname{ker}\left(D_{i}\right)$,

$$
\begin{aligned}
Y_{j}(b \# 1 \cdot 1 \# y) & =Y_{j}(b \# y)=q_{i i}^{m_{i j}} q_{j i} \hat{z}_{m_{i j}}^{(i j)}(b) \# y \\
& =b \# 1 \cdot Y_{j}(1 \# y)+Y_{j}(b \# 1) \cdot\left(g_{i}^{m_{i j}} g_{j} \cdot(1 \# y)\right),
\end{aligned}
$$


where we use that $Y_{j}(1 \# y)=0$. Since,

$$
b_{1} \# 1 \cdot b_{2} \# y^{t}=b_{1} \# 1 \cdot b_{2} \# 1 \cdot(1 \# y)^{t},
$$

(2-12) is valid for products of this form. To prove it in the general case, note that

$$
\left(b_{1} \# y^{t}\right) \cdot\left(b_{2} \# y^{s}\right)=\left(b_{1} \# 1\right) \cdot(1 \# y)^{t} \cdot\left(b_{2} \# y^{s}\right) .
$$

At this point, we have to prove (2-12) for $b \in \mathscr{K}_{i} \operatorname{ker}\left(D_{i}\right), s \in \mathbb{N}$ :

$$
\begin{aligned}
Y_{j}(1 \# & \left.y \cdot b \# y^{s}\right) \\
& =Y_{j}\left(F_{i}(b) \# y^{s}+\left(e_{i} \triangleright b \# y\right) \cdot 1 \# y\right) \\
& =q_{i i}^{m_{i j} s} q_{j i}^{s} \lambda_{i j}^{-1} \hat{z}_{m_{i j}}^{(i j)}\left(F_{i}(b)\right) \# y^{s}+q_{i i}^{m_{i j}(s+1)} q_{j i}^{s+1} \lambda_{i j}^{-1} \cdot \hat{z}_{m_{i j}}^{(i j)}\left(e_{i} \triangleright b\right) \# y^{s+1} \\
& =F_{i}\left(q_{i i}^{m_{i j}(s+1)} q_{j i}^{s+1} \lambda_{i j}^{-1} \hat{z}_{m_{i j}}^{(i j)}(b)\right) \# y^{s}+q_{i i}^{m_{i j}} q_{j i}\left(e_{i} \triangleright\left(q_{i i}^{m_{i j} s} q_{j i}^{s} \lambda_{i j}^{-1} \hat{z}_{m_{i j}}^{(i j)}(b)\right) \# y^{s}\right) \\
& =(1 \# y) \cdot Y_{j}\left(b \# y^{s}\right) \\
& =1 \# y \cdot Y_{j}\left(b \# y^{s}\right)+Y_{j}(1 \# y) \cdot\left(g_{i}^{m_{i j}} g_{j} \cdot b \# y^{s}\right),
\end{aligned}
$$

where we use that $\hat{z}_{m_{i j}}^{(i j)}\left(e_{i} \triangleright b\right)=q_{i i}^{m_{i j}} q_{j i} e_{i} \triangleright\left(\hat{z}_{m_{i j}}^{(i j)}(b)\right)$.

(ii) The algebra $H_{i}$ is $\mathbb{Z}^{\theta}$-graded, with

$$
\operatorname{deg} y=-\mathbf{e}_{i}, \quad \operatorname{deg} e_{i}^{ \pm 1}=0 .
$$

Since $\mathfrak{B}$ and $H_{i}$ are graded and (2-10) holds, the algebra $\mathscr{A}_{i}$ is $\mathbb{Z}^{\theta}$-graded.

Consider the abstract basis $\left\{u_{j}\right\}_{j \in\{1, \ldots, \theta\}}$ of $V_{i}$. With the grading $\operatorname{deg} u_{j}=\mathbf{e}_{j}$, the algebra $\mathfrak{B}\left(V_{i}\right)$ is $\mathbb{Z}^{\theta}$-graded. Consider also the algebra homomorphism $\Omega$ : $T\left(V_{i}\right) \rightarrow s_{i}(\mathfrak{B})$ given by

$$
\Omega\left(u_{j}\right):= \begin{cases}\left(\operatorname{ad}_{c} x_{i}\right)^{m_{i j}}\left(x_{j}\right) & \text { if } j \neq i \\ y & \text { if } j=i .\end{cases}
$$

By part (i) of the theorem, $\Omega$ is an epimorphism, so it induces an isomorphism between $s_{i}(\mathfrak{B})^{\prime}:=T\left(V_{i}\right) / \operatorname{ker} \Omega$ and $s_{i}(\mathfrak{B})$, which we also denote by $\Omega$. We have

$$
\begin{aligned}
& \operatorname{deg} \Omega\left(u_{j}\right)=\operatorname{deg}\left(\left(\operatorname{ad}_{c} x_{i}\right)^{m_{i j}}\left(x_{j}\right)\right)=\mathbf{e}_{j}+m_{i j} \mathbf{e}_{i}=s_{i}\left(\operatorname{deg} \mathbf{u}_{j}\right) \text { if } j \neq i, \\
& \operatorname{deg} \Omega\left(u_{i}\right)=\operatorname{deg}(y)=-\mathbf{e}_{i}=s_{i}\left(\operatorname{deg} \mathbf{u}_{i}\right) .
\end{aligned}
$$

Since $\Omega$ is an algebra homomorphism, we have $\operatorname{deg}(\Omega(\mathbf{u}))=s_{i}(\operatorname{deg}(\mathbf{u}))$ for all $\mathbf{u} \in s_{i}(\mathfrak{B})^{\prime}$. Since $s_{i}^{2}=\mathrm{id}, s_{i}(\operatorname{deg}(\Omega(\mathbf{u})))=\operatorname{deg}(\mathbf{u})$ for all $\mathbf{u} \in s_{i}(\mathfrak{B})^{\prime}$, and $\mathfrak{H}_{s_{i}(\mathfrak{B})^{\prime}}=$ $s_{i}\left(\mathfrak{H}_{s_{i}(\mathfrak{B})}\right)$.

From this point on, the proof goes exactly as in [Andruskiewitsch and Angiono 2008, Theorem 3.2]. 
(iii) This is Proposition 1 in [Heckenberger 2006b].

By Theorem 2.6, the initial braided vector space with matrix $\left(q_{k j}\right)_{1 \leq k, j \leq \theta}$ is transformed into another braided vector space of diagonal type $V_{i}$, with matrix $\left(\tilde{q}_{k j}\right)_{1 \leq k, j \leq \theta}$, where $\tilde{q}_{j k}=q_{i i}^{m_{i j} m_{i k}} q_{i k}^{m_{i j}} q_{j i}^{m_{i k}} q_{j k}$ for $j, k \in\{1, \ldots, \theta\}$.

If $j \neq i$, then $\widetilde{m}_{i j}=\min \left\{m \in \mathbb{N}:(m+1) \tilde{q}_{i i}\left(\tilde{q}_{i i}^{m} \tilde{q}_{i j} \tilde{q}_{j i}=0\right)\right\}=m_{i j}$.

For later use in Section 5, we recall a result from [Andruskiewitsch et al. 2008], adapted to diagonal braided vector spaces.

Lemma 2.7 [Andruskiewitsch et al. 2008, Lemma 2.8(ii)]. Let $V$ be a diagonal braided vector space and $I$ a $\mathbb{Z}^{\theta}$-homogeneous ideal of $T(V)$. Set $\mathfrak{B}:=T(V) / I$ and assume that for all $i \in\{1, \ldots, \theta\}$ there exist (id, $\left.\sigma_{i}\right)$-derivations $D_{i}: \mathfrak{B} \rightarrow \mathfrak{B}$ with $D_{i}\left(x_{j}\right)=\delta_{i, j}$ for all $j$. Then $I \subseteq I(V)$.

That is, the canonical surjective algebra morphisms from $T(V)$ onto $\mathfrak{B}$ and $\mathfrak{B}(V)$ induce a surjective algebra morphism $\mathfrak{B} \rightarrow \mathfrak{B}(V)$.

\section{Standard braidings}

Heckenberger [2006a] has classified diagonal braidings whose set of PBW generators is finite. Standard braidings form an special subclass, which includes properly braidings of Cartan type.

We first recall the definition of a standard braiding from [Andruskiewitsch and Angiono 2008], and the notion of a Weyl groupoid, introduced in [Heckenberger 2006b]. Then we present the classification of standard braidings, and compare them with [Heckenberger 2006a].

Like Heckenberger, we use the generalized Dynkin diagram associated to a braided vector space of diagonal type, with matrix $\left(q_{i j}\right)_{1 \leq i, j \leq \theta}$ : this is a graph with $\theta$ vertices, each labeled with the corresponding $q_{i i}$, and an edge between two vertices $i, j$ labeled with $q_{i j} q_{j i}$ if this scalar is different from 1 . So two braided vector spaces of diagonal type have the same generalized Dynkin diagram if and only if they are twist equivalent. We shall assume that the generalized Dynkin diagram is connected, by [Andruskiewitsch and Schneider 2000, Lemma 4.2].

Summarizing, the main result of this section says:

Theorem 3.1. Any standard braiding is twist equivalent with one or more of

- a braiding of Cartan type,

- a braiding of type $A_{\theta}$ listed in Proposition 3.9,

- a braiding of type $B_{\theta}$ listed in Proposition 3.10, or

- a braiding of type $G_{2}$ listed in Proposition 3.11. 
The generalized Dynkin diagrams appearing in Propositions 3.9 and 3.10 correspond to rows 1, 2, 3, 4, 5, 6 in [Heckenberger 2006a, Table C]. The generalized Dynkin diagrams in Proposition 3.11 are (T8) in [Heckenberger 2008, Section 3]. However, our classification does not rely on Heckenberger's papers.

3A. The Weyl groupoid and standard braidings. Let $E=\left(\mathbf{e}_{1}, \ldots, \mathbf{e}_{\theta}\right)$ be the canonical basis of $\mathbb{Z}^{\theta}$. Consider an arbitrary matrix $\left(q_{i j}\right)_{1 \leq i, j \leq \theta} \in\left(\mathrm{k}^{\times}\right)^{\theta \times \theta}$, and fix once and for all the bilinear form $\chi: \mathbb{Z}^{\theta} \times \mathbb{Z}^{\theta} \rightarrow \mathrm{k}^{\times}$determined by

$$
\chi\left(\mathbf{e}_{i}, \mathbf{e}_{j}\right)=q_{i j}, \quad 1 \leq i, j \leq \theta .
$$

If $F=\left(\mathbf{f}_{1}, \ldots, \mathbf{f}_{\theta}\right)$ is another ordered basis of $\mathbb{Z}^{\theta}$, then we set $\tilde{q}_{i j}=\chi\left(\mathbf{f}_{i}, \mathbf{f}_{j}\right)$, $1 \leq i, j \leq \theta$. We call $\left(\tilde{q}_{i j}\right)$ the braiding matrix with respect to the basis $F$. Fix $i \in\{1, \ldots, \theta\}$. If $1 \leq i, j \leq \theta$, we consider the set

$$
\widetilde{M}_{i j}:=\left\{m \in \mathbb{N}_{0}:(m+1)_{\tilde{q}_{i i}}\left(\tilde{q}_{i i}^{m} \tilde{q}_{i j} \tilde{q}_{j i}-1\right)=0\right\} .
$$

If this set is nonempty, its minimal element is denoted $\tilde{m}_{i j}$ (which of course depends on the basis $F$ ). Define also $\tilde{m}_{i i}=2$. Let $s_{i, F} \in \mathrm{GL}\left(\mathbb{Z}^{\theta}\right)$ be the pseudoreflection given by $s_{i, F}\left(\mathbf{f}_{j}\right):=\mathbf{f}_{j}+\tilde{m}_{i j} \mathbf{f}_{i}$, for $j \in\{1, \ldots, \theta\}$.

Let $G$ be a group acting on a set $X$. We define the transformation groupoid as $G \times X$ with the operation given by $(g, x)(h, y)=(g h, y)$ if $x=h(y)$, but undefined otherwise.

Definition 3.2. Consider the set $\mathfrak{X}$ of all ordered bases of $\mathbb{Z}^{\theta}$, and the canonical action of $\operatorname{GL}\left(\mathbb{Z}^{\theta}\right)$ over $\mathfrak{X}$. The Weyl groupoid $W(\chi)$ of the bilinear form $\chi$ is the smallest subgroupoid of the transformation groupoid $\operatorname{GL}\left(\mathbb{Z}^{\theta}\right) \times \mathfrak{X}$ that satisfies following properties:

- (id, $E) \in W(\chi)$,

- if (id, $F) \in W(\chi)$ and $s_{i, F}$ is defined, then $\left(s_{i, F}, F\right) \in W(\chi)$.

Let $\mathfrak{P}(\chi)=\{F:(\mathrm{id}, F) \in W(\chi)\}$ be the set of points of the groupoid $W(\chi)$. The set

$$
\Delta(\chi)=\bigcup_{F \in \mathfrak{P}(\chi)} F
$$

is called the generalized root system ${ }^{1}$ associated to $\chi$.

We record for later use the following evident facts.

Remark 3.3. Take $i \in\{1, \ldots, \theta\}$ such that $s_{i, E}$ is defined. Set $F=s_{i, E}(E)$ and let $\left(\tilde{q}_{i j}\right)$ be the braiding matrix with respect to the basis $F$. Assume that

\footnotetext{
${ }^{1}$ Following the traditional notation in the theory of Lie algebras, we should speak about systems of real roots, since in the case of braidings of symmetrizable Cartan type one would get just the real roots. But we prefer to follow the denomination in [Andruskiewitsch and Angiono 2008]
} 
- $q_{i i}=-1$ (so $m_{i k}=0$ if $q_{i k} q_{k i}=1$ or $m_{i k}=1$, for each $k \neq i$ );

- there exists $j \neq i$ such that $q_{j j} q_{j i} q_{i j}=1$ (that is, $m_{i j}=m_{j i}=1$ ).

Then $\tilde{q}_{j j}=-1$.

Proof. Simply, $\tilde{q}_{j j}=q_{i i} q_{i j} q_{j i} q_{j j}=q_{i i}=-1$.

Remark 3.4. If the $m_{i j}$ satisfy $q_{i i}^{m_{i j}} q_{i j} q_{j i}=1$ for all $j \neq i$, the braiding of $V_{i}$ is twist equivalent with the corresponding to $V$.

Define $\alpha: W(\chi) \rightarrow \operatorname{GL}(\theta, \mathbb{Z})$ by $\alpha(s, F)=s$ if $(s, F) \in W(\chi)$, and denote by $W_{0}(\chi)$ the subgroup generated by the image of $\alpha$.

Definition 3.5. [Andruskiewitsch and Angiono 2008] We say that $\chi$ is standard if for any $F \in \mathfrak{P}(\chi)$, the integers $m_{r j}$ are defined, for all $1 \leq r, j \leq \theta$, and the integers $m_{r j}$ for the bases $s_{i, F}(F)$ coincide with those for $F$ for all $i, r, j$. Clearly it is enough to assume this for the canonical basis $E$.

We now assume that $\chi$ is standard. We set $C:=\left(a_{i j}\right) \in \mathbb{Z}^{\theta \times \theta}$, where $a_{i j}=-m_{i j}$; this is a generalized Cartan matrix.

Proposition 3.6 [Andruskiewitsch and Angiono 2008]. $W_{0}(\chi)=\left\langle s_{i, E}: 1 \leq i \leq \theta\right\rangle$. Furthermore $W_{0}(\chi)$ acts freely and transitively on $\mathfrak{P}(\chi)$.

Hence, $W_{0}(\chi)$ is a Coxeter group, and $W_{0}(\chi)$ and $\mathfrak{P}(\chi)$ have the same cardinality.

Lemma 3.7 [Andruskiewitsch and Angiono 2008]. The following are equivalent:

(1) The groupoid $W(\chi)$ is finite.

(2) The set $\mathfrak{P}(\chi)$ is finite.

(3) The generalized root system $\Delta(\chi)$ is finite.

(4) The group $W_{0}(\chi)$ is finite.

(5) The Cartan matrix $C$ is symmetrizable and of finite type.

We shall prove in Theorem 4.1, that if $\Delta(\chi)$ is finite, the matrix $C$ is symmetrizable, hence of finite type. Thus $\mathfrak{B}(V)$ is of finite dimension if and only if the Cartan matrix $C$ is of finite type.

3B. Classification of standard braidings. We now classify standard braidings such that the Cartan matrix is of finite type. We begin with types $C_{\theta}, D_{\theta}, E_{l}(l=6,7,8)$ and $F_{4}$ : these standard braidings are necessarily of Cartan type.

Proposition 3.8. Let $V$ be a braided vector space of standard type, $\operatorname{set} \theta=\operatorname{dim} V$, and let $C=\left(a_{i j}\right)_{i, j \in\{1, \ldots, \theta\}}$ be the corresponding Cartan matrix, of type $C_{\theta}, D_{\theta}$, $E_{l}(l=6,7,8)$ or $F_{4}$. Then $V$ is of Cartan type (associated to the corresponding matrix of finite type). 
Proof. Let $V$ be standard of type $C_{\theta}, \theta \geq 3$.

$$
\circ^{1}-\circ^{2}-\circ^{3} \ldots \quad \circ^{\theta-2}-\circ^{\theta-1} \Longleftarrow \circ^{\theta}
$$

Note that $q_{\theta-1, \theta-1} \neq-1$ by Remark 3.3 and the assumption $m_{\theta-1, \theta}=2$. Since $m_{\theta-1, \theta-2}=1, q_{\theta-1, \theta-1} q_{\theta-1, \theta-2} q_{\theta-2, \theta-1}=1$. Using Remark 3.3 when $i=\theta-$ $2, j=\theta-1$, since $\tilde{q}_{\theta-1, \theta-1} \neq-1$ when we transform by $s_{\theta-2}$ (since the new braided vector space is also standard), we have $q_{\theta-2, \theta-2} \neq-1$, so

$$
q_{\theta-2, \theta-2} q_{\theta-2, \theta-1} q_{\theta-1, \theta-2}=q_{\theta-2, \theta-2} q_{\theta-2, \theta-3} q_{\theta-3, \theta-2}=1,
$$

and $q_{\theta-1, \theta-1}=q_{\theta-2, \theta-2}$. Inductively,

$$
q_{k k} q_{k, k-1} q_{k-1, k}=q_{k k} q_{k, k+1} q_{k+1, k}=q_{11} q_{12} q_{21}=1, \quad k=2, \ldots, \theta-1
$$

and $q_{11}=q_{22}=\ldots=q_{\theta-1, \theta-1}$. So we look at $q_{\theta \theta}$ : since $m_{\theta, \theta-1}=1$, we have $q_{\theta \theta}=-1$ or $q_{\theta \theta} q_{\theta, \theta-1} q_{\theta-1, \theta}=1$. If $q_{\theta \theta}=-1$, transforming by $s_{\theta}$, we have

$$
\tilde{q}_{\theta-1, \theta-1}=-q^{-1}, \quad \tilde{q}_{\theta-1, \theta} \tilde{q}_{\theta, \theta-1}=q^{2},
$$

and $q^{2}=-1$ since $m_{\theta-1, \theta-2}=1$. Then

$$
q_{\theta \theta} q_{\theta, \theta-1} q_{\theta-1, \theta}=1, \quad q_{\theta \theta}=q^{2},
$$

and the braiding is of Cartan type in both cases.

Let $V$ be standard of type $D_{\theta}, \theta \geq 4$.

We prove the statement by induction on $\theta$. Let $V$ be standard of type $D_{4}$, and suppose that $q_{22}=-1$. Let $\left(\tilde{q}_{i j}\right)$ the braiding matrix with respect to $F=s_{2, E}(E)$. We calculate for each pair $j \neq k \in\{1,3,4\}$ :

$$
\tilde{q}_{j k} \tilde{q}_{k j}=\left((-1) q_{2 k} q_{j 2} q_{j k}\right)\left((-1) q_{2 j} q_{k 2} q_{k j}\right)=\left(q_{2 k} q_{k 2}\right)\left(q_{2 j} q_{j 2}\right),
$$

where we use that $q_{j k} q_{k j}=1$. Since also $\tilde{q}_{j k} \tilde{q}_{k j}=1$, we have $q_{2 k} q_{k 2}=\left(q_{2 j} q_{j 2}\right)^{-1}$ for $j \neq k$, so $q_{2 k} q_{k 2}=-1, k=1,3,4$, since $q_{2 k} q_{k 2} \neq 1$. In this case, the braiding is of Cartan type, with $q=-1$. Suppose then $q_{22} \neq-1$. From the fact that $m_{2 j}=1$, we have

$$
q_{22} q_{2 j} q_{j 2}=1, \quad j=1,3,4 \text {. }
$$

For each $j$, applying Remark 3.3, we see that $q_{j j} \neq-1$ (since $\tilde{q}_{22} \neq-1$ ), so $q_{j j} q_{2 j} q_{j 2}=1$, for $j=1,3,4$, and the braiding is of Cartan type.

$$
\circ^{1}-\circ^{2}-\circ^{3} \ldots \quad \circ^{\theta-2}-\circ^{\theta}
$$


We now suppose the statement valid for $\theta$. Let $V$ be a standard braided vector space of type $D_{\theta+1}$. The subspace generated by $x_{2}, \ldots, x_{\theta+1}$ is a standard braided vector space associated to the matrix $\left(q_{i j}\right)_{i, j=2, \ldots, \theta+1}$, of type $D_{\theta}$, so it is of Cartan type. To finish, apply Remark 3.3 with $i=1, j=2$, to conclude that $V$ is of Cartan type with $q=-1$, or, if $q_{22} \neq-1$, we have $q_{11} \neq-1$ and $q_{11} q_{12} q_{21}=1$, and in this case it is of Cartan type too (because also $q_{1 k} q_{k 1}=1$ when $k>2$ ).

Let $V$ be standard of type $E_{6}$. Note that $1,2,3,4,5$ determine a braided vector subspace, which is standard of type $D_{5}$, hence of Cartan type. To prove that $q_{66} q_{65} q_{56}=1$, we use Remark 3.3 as above.

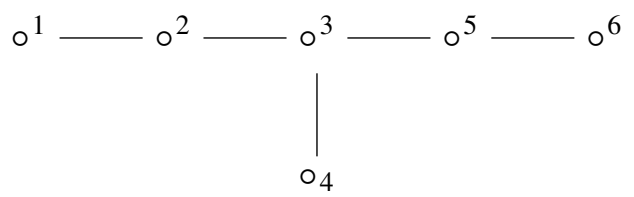

If $V$ is standard of type $E_{7}$ or $E_{8}$, we proceed similarly by reduction to $E_{6}$ or $E_{7}$, respectively.

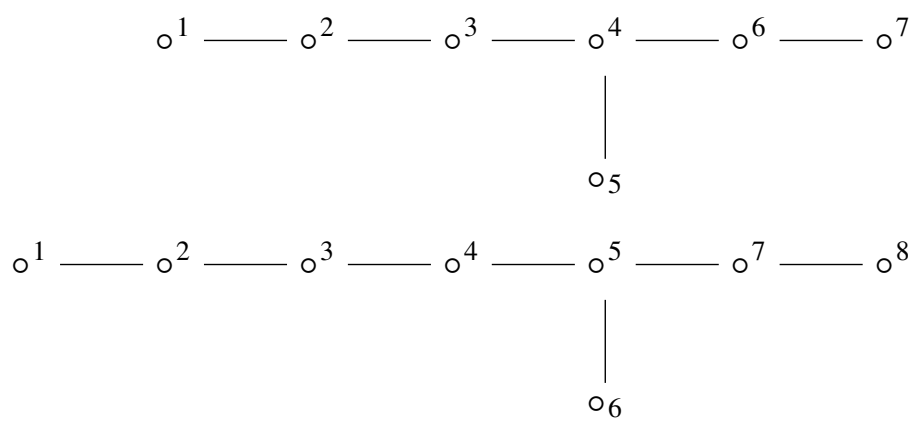

Let $V$ be standard of type $F_{4}$. Vertices 2, 3, 4 determine a braided subspace, which is standard of type $C_{3}$, so the $q_{i j}$ satisfy the corresponding relations. Let $\left(\tilde{q}_{i j}\right)$ the braiding matrix with respect to $F=s_{2, E}(E)$. Since $\tilde{q}_{13} \tilde{q}_{31}=1$ and $q_{22} q_{23} q_{32}=1$, we have $q_{22} q_{12} q_{21}=1$.

$$
\circ^{1} \longrightarrow \circ^{2} \Longrightarrow \circ^{3}-\circ^{4}
$$

Now, if we suppose $q_{11}=-1$, applying Remark 3.3 we have $q_{22}=-1=q_{21} q_{12}$, and the corresponding vector space is of Cartan type $F_{4}$, associated to $q \in \mathbb{G}_{4}$. If $q_{11} \neq-1$, then $q_{11} q_{12} q_{21}=1$, and the space it again is of Cartan type.

To finish the classification of standard braidings, we describe the standard braidings that are not of Cartan type. They are associated to Cartan matrices of type $A_{\theta}, B_{\theta}$ or $G_{2}$. 
We use a notation similar to the one in [Heckenberger 2006a] for a special kind of braiding of type $A_{\theta}$ (here we emphasize the positions where $q_{i i}=-1$, which we use to compute the dimension of the corresponding Nichols algebra); $\mathscr{C}\left(\theta, q ; i_{1}, \ldots, i_{j}\right)$ corresponds to the generalized Dynkin diagram

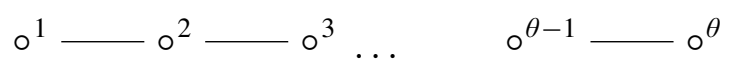

where the following equations hold:

- $q=q_{\theta-1, \theta} q_{\theta, \theta-1} q_{\theta \theta}^{2}$,

- $\left(q_{\theta \theta}+1\right)\left(q_{\theta \theta} q_{\theta-1, \theta} q_{\theta, \theta-1}-1\right)=\left(q_{11}+1\right)\left(q_{11} q_{12} q_{21}-1\right)=0$;

- $-q_{i i}=q_{i-1, i} q_{i, i-1} q_{i+1, i} q_{i, i+1}=1$ if $i \in\left\{i_{1}, \ldots, i_{j}\right\}$.

- $q_{i i} q_{i-1, i} q_{i, i-1}=q_{i i} q_{i+1, i} q_{i, i+1}=1$, otherwise.

Then $q_{i i}=-1$ if and only if $q_{i-1, i} q_{i, i-1}=\left(q_{i+1, i} q_{i, i+1}\right)^{-1}$.

Proposition 3.9. Let $V$ be a braided vector space of diagonal type. Then $V$ is standard of type $A_{\theta}$ if and only if its generalized Dynkin diagram is of the form

$$
\mathscr{C}\left(\theta, q ; i_{1}, \ldots, i_{j}\right) \text {. }
$$

This braiding is of Cartan type if and only if $j=0$, or $j=n$ with $q=-1$.

Proof. Let $V$ be a braided vector space of standard $A_{\theta}$ type. For each vertex $i$, with $1<i<\theta$, we have $q_{i i}=-1$ or $q_{i i} q_{i, i-1} q_{i-1, i}=q_{i i} q_{i, i+1} q_{i+1, i}=1$, and similar formulas hold for $i=1, \theta$. So suppose that $1<i<\theta$ and $q_{i i}=-1$. We transform by $s_{i}$ and obtain

$$
\tilde{q}_{i-1, i+1}=-q_{i, i+1} q_{i-1, i} q_{i-1, i+1}, \quad \tilde{q}_{i+1, i-1}=-q_{i, i-1} q_{i+1, i} q_{i+1, i-1},
$$

and using that $m_{i-1, i+1}=\widetilde{m}_{i-1, i+1}=0$, we have

$$
q_{i-1, i+1} q_{i+1, i-1}=1, \quad \tilde{q}_{i-1, i+1} \tilde{q}_{i+1, i-1}=1,
$$

so we deduce that $q_{i, i+1} q_{i+1, i}=\left(q_{i, i-1} q_{i-1, i}\right)^{-1}$. Then the corresponding matrix $\left(q_{i j}\right)$ is of the form (3-10).

Now consider $V$ of the form (3-10). Assume $q_{i i}=q^{ \pm 1}$; if we transform by $s_{i}$, the braided vector space $V_{i}$ is twist equivalent with $V$ by Remark 3.4. Thus, $\widetilde{m}_{i j}=m_{i j}$.

Assume $q_{i i}=-1$. We transform by $s_{i}$ and calculate

$$
\tilde{q}_{j j}=(-1)^{m_{i j}^{2}}\left(q_{i j} q_{j i}\right)^{m_{i j}} q_{j j}= \begin{cases}q_{j j} & \text { if }|j-i|>1, \\ (-1) q^{\mp 1} q^{ \pm 1}=-1 & \text { if } j=i \pm 1, q_{j j}=q^{ \pm 1} \\ (-1) q^{ \pm 1}(-1)=q^{ \pm 1} & \text { if } j=i \pm 1, q_{j j}=-1\end{cases}
$$


Also, $\tilde{q}_{i j} \tilde{q}_{j i}=q_{i j} q_{j i}$ if $|j-i|>1$ and $\tilde{q}_{i j} \tilde{q}_{j i}=q_{i j}^{-1} q_{j i}^{-1}$ if $|j-i|=1$; moreover

$$
\tilde{q}_{k j} \tilde{q}_{j k}=\left(q_{i k} q_{k i}\right)^{m_{i j}}\left(q_{i j} q_{j i}\right)^{m_{i k}} q_{k j} q_{j k}= \begin{cases}q_{k j} q_{j k} & \text { if }|j-i| \text { or }|k-i|>1, \\ 1 & \text { if } j=i-1, k=i+1 .\end{cases}
$$

Then $V_{i}$ has a braiding of the above form too, and $\left(-m_{i j}\right)$ corresponds to the finite Cartan matrix of type $A_{\theta}$, so it is a standard braiding of type $A_{\theta}$. Thus this is the complete family of standard braidings of type $A_{\theta}$.

Proposition 3.10. Let $V$ a diagonal braided vector space. Then $V$ is standard of type $B_{\theta}$ if and only if its generalized Dynkin diagram is of one of these forms:

$$
\zeta q^{-1} q \text { with } \zeta \in \mathbb{G}_{3}, q \neq \zeta(\theta=2)
$$

(b)

$$
\begin{aligned}
& \text { (b) } \mathscr{C}\left(\theta-1, q^{2} ; i_{1}, \ldots, i_{j}\right)-q^{-2} q \text { with } q \neq 0,-1, \quad 0 \leq j \leq \theta-1 \text {; } \\
& \text { (c) } \mathscr{C}\left(\theta-1,-\zeta^{-1} ; i_{1}, \ldots, i_{j}\right)-\zeta \zeta \text { with } \zeta \in \mathbb{G}_{3}, \quad 0 \leq j \leq \theta-1 .
\end{aligned}
$$

This braiding is of Cartan type if and only if it is as in (b) and $j=0$.

Proof. First we analyze the case $\theta=2$. Let $V$ a standard braided vector space of type $B_{2}$. There are several possibilities:

- $q_{11}^{2} q_{12} q_{21}=q_{22} q_{21} q_{12}=1$ : this braiding is of Cartan type, with $q=q_{11}$. Note that $q \neq-1$. This braiding has the form (b) with $\theta=2, j=0$.

- $q_{11}^{2} q_{12} q_{21}=1, q_{22}=-1$. We transform by $s_{2}$, obtaining

$$
\tilde{q}_{11}=-q_{11}^{-1}, \quad \tilde{q}_{12} \tilde{q}_{21}=q_{12}^{-1} q_{21}^{-1} .
$$

Thus $\tilde{q}_{11}^{2} \tilde{q}_{12} \tilde{q}_{21}=1$. It has the form (b) with $j=1$.

- $q_{11} \in \mathbb{G}_{3}, q_{22} q_{21} q_{12}=1$. We transform by $s_{1}$, obtaining

$$
\tilde{q}_{22}=q_{11} q_{12} q_{21}, \quad \tilde{q}_{12} \tilde{q}_{21}=q_{11}^{2} q_{12}^{-1} q_{21}^{-1} .
$$

So $\tilde{q}_{22} \tilde{q}_{21} \tilde{q}_{12}=1$, which is the case (a).

- $q_{11} \in \mathbb{G}_{3}, q_{22}=-1$ : we transform by $s_{1}$, obtaining

$$
\tilde{q}_{22}=-q_{12}^{2} q_{21}^{2} q_{11}, \quad \tilde{q}_{12} \tilde{q}_{21}=q_{11}^{2} q_{12}^{-1} q_{21}^{-1} .
$$

If we transform by $s_{2}$,

$$
\tilde{q}_{11}=-q_{12} q_{21} q_{11}, \quad \tilde{q}_{12} \tilde{q}_{21}=q_{12}^{-1} q_{21}^{-1} .
$$

So $q_{12} q_{21}= \pm q_{11}$, and we discard the case $q_{12} q_{21}=q_{11}$ because it has been considered before. The braiding has the form (c) with $j=0$, and is standard. 
Conversely, all braidings (a), (b) and (c) are standard of type $B_{2}$.

Now let $V$ be of type $B_{\theta}$, with $\theta \geq 3$. The first $\theta-1$ vertices determine a braiding of standard type $A_{\theta-1}$, and the last two determine a braiding of standard type $B_{2}$; so we have to glue the possible such braidings. The possible cases are the two presented in Proposition 3.10, plus

$$
\mathscr{C}\left(\theta-2, q ; i_{1}, \ldots, i_{j}\right) q^{-1} \stackrel{q}{O} q^{-1} q^{\zeta} .
$$

But if we transform by $s_{\theta}$, we obtain

$$
\tilde{q}_{\theta-1, \theta-1}=\zeta q^{-1}, \quad \tilde{q}_{\theta-1, \theta-2} \tilde{q}_{\theta-2, \theta-1}=q^{-1},
$$

so $1=\tilde{q}_{\theta-1, \theta-1} \tilde{q}_{\theta-1, \theta-2} \tilde{q}_{\theta-2, \theta-1}$ and we obtain $q= \pm \zeta^{-1}$, or $\tilde{q}_{\theta-1, \theta-1}=-1$. Then $q=-\zeta^{-1}$ or $q=-1$, so it is of some of the above forms.

To prove that (b) and (c) are standard braidings, we use the following fact: if $m_{i j}=0$ (that is, $q_{i j} q_{j i}=1$ ) and we transform by $s_{i}$, then

$$
\tilde{q}_{j j}=q_{j j} \quad \text { and } \quad \tilde{q}_{j k} \tilde{q}_{j k}=q_{j k} q_{k j} \quad \text { for } k \neq i .
$$

In this case, $m_{i j}=0$ if $|i-j|>1$; if, on the contrary, $j=i \pm 1$, we use the fact that the subdiagram determined by these two vertices is standard of type $B_{2}$ or type $A_{2}$. So this is the complete family of all twist equivalence classes of standard braidings of type $B_{\theta}$.

Proposition 3.11. Let $V$ a braided vector space of diagonal type. Then $V$ is standard of type $G_{2}$ if and only if its generalized Dynkin diagram is one of the following:

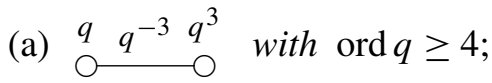

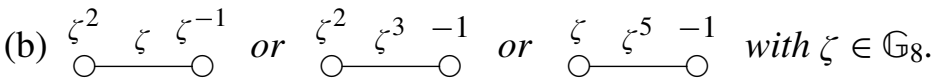

This braiding is of Cartan type if and only if it is as in (a).

Proof. Let $V$ be a standard braiding of type $G_{2}$. There are four possible cases:

- $q_{11}^{3} q_{12} q_{21}=1, q_{22} q_{21} q_{12}=1$ : this braiding is of Cartan type, as in (a), with $q=q_{11}$. If $q$ is a root of unity, then ord $q \geq 4$ because $m_{12}=3$.

- $q_{11}^{3} q_{12} q_{21}=1, q_{22}=-1$ : we transform by $s_{2}$, obtaining

$$
\tilde{q}_{11}=-q_{11}^{-2}, \quad \tilde{q}_{12} \tilde{q}_{21}=q_{12}^{-1} q_{21}^{-1} .
$$

If $1=\tilde{q}_{11}^{3} \tilde{q}_{12} \tilde{q}_{21}=-q_{11}^{-3}$, then $q_{12} q_{21}=-1$, and the braiding is of Cartan type with $q_{11} \in \mathbb{G}_{6}$. If not, $1=\tilde{q}_{11}^{4}=q_{11}^{-8}$ and ord $\tilde{q}_{11}=4$, so ord $q_{11}=8$. Then we can express the braiding in the form of the third diagram in (b). 
- $q_{11} \in \mathbb{G}_{4}, q_{22} q_{21} q_{12}=1$ : we transform by $s_{1}$, obtaining

$$
\tilde{q}_{22}=q_{11} q_{12}^{2} q_{21}^{2}, \quad \tilde{q}_{12} \tilde{q}_{21}=-q_{12}^{-1} q_{21}^{-1} .
$$

If $1=\tilde{q}_{22} \tilde{q}_{21} \tilde{q}_{12}=-q_{11} q_{12} q_{21}$, we have $q_{11}^{3} q_{12} q_{21}=1$ because $q_{11}^{2}=-1$, and this is a braiding of Cartan type. So consider now the case $-1=\tilde{q}_{22}=q_{11} q_{12}^{2} q_{21}^{2}$, from which $q_{22}^{2}=q_{11}^{-1}$ and $q_{22} \in \mathbb{G}_{8}$. Then we obtain a braiding of the form of the first diagram in (b).

- $q_{11} \in \mathbb{G}_{4}, q_{22}=-1:$ we transform by $s_{2}$, obtaining

$$
\tilde{q}_{11}=-q_{12} q_{21} q_{11}, \quad \tilde{q}_{12} \tilde{q}_{21}=q_{12}^{-1} q_{21}^{-1} .
$$

If $\tilde{q}_{11} \in \mathbb{G}_{4}$, then $\left(q_{12} q_{21}\right)^{4}=1$. Moreover $q_{12} q_{21} \neq 1$ and $q_{12} q_{21} \neq q_{11}^{-1}$ because $m_{12}=3$. So $q_{12} q_{21}=-1$ or $q_{12} q_{21}=q_{11}=q_{11}^{-3}$; but these cases have been considered already. There remains to analyze the case

$$
1=\tilde{q}_{11}^{3} \tilde{q}_{12} \tilde{q}_{21}=q_{11} q_{12}^{2} q_{21}^{2},
$$

which we can express in the form of the second diagram in (b), for some $\zeta \in \mathbb{G}_{8}$. A simple calculation proves that these braidings are of standard type, so they are all the standard braidings of type $G_{2}$.

\section{Nichols algebras of standard braided vector spaces}

In this section we study Nichols algebras associated to standard braidings. We assume that the Dynkin diagram is connected, as in Section 3. In Section 4A we prove that the set $\Delta^{+}(\mathfrak{B}(V))$ is in bijection with $\Delta_{C}^{+}$, the set of positive roots associated with the finite Cartan matrix $C$.

We describe an explicit set of generators in Section 4B, following [Lalonde and Ram 1995]. We adapt their proof since they work on enveloping algebras of simple Lie algebras. In Section 4C, we calculate the dimension of Nichols algebra associated to a standard braided vector space, type by type.

4A. PBW bases of Nichols algebras. We start with a result analogous to [Heckenberger 2006b, Theorem 1], but for braidings of standard type.

Theorem 4.1. Let $V$ be a braided vector space of standard type with Cartan matrix $C$. Then the set $\Delta(\mathfrak{B}(V))$ is finite if and only if the Cartan matrix $C$ is symmetrizable and of finite type.

Proof. Since we assume $V$ of standard type, $\Delta(\mathfrak{B}(V))$ coincides with the set of real roots corresponding to the matrix $C$ by [Heckenberger 2006b, Proposition 1], where we identify corresponding simple roots. Hence, if $C$ is not symmetrizable or not of finite type, the set of real roots is infinite by the classification of finite Coxeter groups, and hence $\Delta(\mathfrak{B}(V))$ is infinite. 
Conversely, let $C$ be symmetrizable and of finite type. Then the set of real roots is finite. Take $\alpha \in \Delta(\mathfrak{B}(V))$ and let $k \in \mathbb{N}, i_{1}, \ldots, i_{k} \in\{1, \ldots, \theta\}$ be a sequence of integers such that $s_{i_{1}} \cdots s_{i_{k}}$ is a longest element in $W_{0}(\chi)$. Since all roots are positive or negative, there exists $l \in\{1, \ldots, k\}$ such that $\beta=s_{i_{l+1}} \cdots s_{i_{k}}(\alpha)$ is positive and $s_{i_{l}}(\beta)$ is negative. But then $\beta=\alpha_{i_{l}}$, and $\alpha=s_{i_{k}} \cdots s_{i_{l+1}}\left(\alpha_{i_{l}}\right)$ is a real root. Thus $\Delta(\mathfrak{B}(V))$ is finite.

Corollary 4.2. Let $V$ be a braided vector space of standard type, set $\theta=\operatorname{dim} V$, and let $C=\left(a_{i j}\right)_{i, j \in\{1, \ldots, \theta\}}$ be the corresponding generalized Cartan matrix of finite type.

(a) $\phi\left(\Delta_{C}\right)=\Delta(\mathfrak{B}(V))$, where as before $\phi: \mathbb{Z} \pi \rightarrow \mathbb{Z}^{\theta}$ is the $\mathbb{Z}$-linear map determined by $\phi\left(\alpha_{i}\right):=\mathbf{e}_{i}$.

(b) The multiplicity of each root in $\Delta(\mathfrak{B}(V))$ is one.

Proof. Statement (a) follows from the proof of Theorem 4.1.

Using this condition, since each root is of the form $\beta=w\left(\alpha_{i}\right)$ for some $w \in W$ and $i \in\{1, \ldots, \theta\}$, we conclude by applying a certain sequence of transformations $s_{i}$ that this is the degree corresponding to a generator of the corresponding Nichols algebra, so the multiplicity (which is invariant under these transformations) is 1 .

4B. Explicit generators for a PBW basis. In view of Corollary 4.2, we restrict our attention to finding one Lyndon word for each positive root of the root system associated with the corresponding finite Cartan matrix.

Proposition 4.3 [Lalonde and Ram 1995, Proposition 2.9]. Let $l$ be an element of $S_{I}$. Then $l$ is of the form $l=l_{1} \ldots l_{k} a$, for some $k \in \mathbb{N}_{0}$, where

- $l_{i} \in S_{I}$ for each $i=1, \ldots, k$;

- $l_{i}$ is a beginning of $l_{i-1}$ for each $i>1$; and

- $a$ is a letter.

Also, if $l=u v$ is the Shirshov decomposition, then $u, v \in S_{I}$.

In what follows, we describe a set of Lyndon words for each Cartan matrix of finite type $C$.

Consider $\alpha=\sum_{j=1}^{\theta} a_{j} \alpha_{j} \in \Delta^{+}$and let $l_{\alpha} \in S_{I}$ be such that $\operatorname{deg} l_{\alpha}=\alpha$. Let $l_{\alpha}=l_{\beta_{1}} \ldots l_{\beta_{k}} x_{s}$ be a decomposition as above, where $s \in\{1, \ldots, \theta\}$ and $\operatorname{deg} l_{\beta_{j}}=\beta_{j}$. Since each $l_{\beta_{j}}$ is a beginning of $l_{\beta_{j-1}}$, all the words begin with the same letter $x^{\prime}$, which satisfies $x^{\prime}<x_{s}$ because $l$ is a Lyndon word. Therefore $x^{\prime}$ is the least letter of $l$, so

$$
x^{\prime}=x_{i}, \quad i=\min \left\{j: a_{j} \neq 0\right\} \quad \Longrightarrow \quad \alpha=\sum_{j=i}^{\theta} a_{j} \alpha_{j}
$$


Then $k \leq a_{i} \leq 3$, for the order given in (3-9), (3-4), (3-5), (3-6), (3-7), (3-8) (the value $a_{i}=3$ appears only when $C$ is of type $G_{2}$ ).

Now, each $l_{\beta_{j}}$ lies in $S_{I}$, so $\beta_{j} \in \Delta^{+}$; i.e., it corresponds to a term of the PBW basis. Also $\sum_{j=1}^{k} \beta_{j}+\alpha_{s}=\alpha$. If $k=2$, we have $\beta_{1}-\beta_{2}=\sum_{j=1}^{\theta} b_{j} \alpha_{j}$ and $b_{j} \geq 0$, because $\beta_{2}$ is a beginning of $\beta_{1}$ (an analogous claim is valid when the matrix is of type $G_{2}$ and $k=3$ ). With these rules we define inductively Lyndon words for a PBW basis corresponding with a standard braiding for a fixed order on the letters. This is done as in [Lalonde and Ram 1995], but taking care that in that reference Serre relations are used; here we have quantum Serre relations, and some quantum binomial coefficients may be zero.

Type $A_{\theta}:$ In this case, the roots are of the form

$$
\mathbf{u}_{i, j}:=\sum_{k=i}^{j} \alpha_{k}, \quad 1 \leq i \leq j \leq \theta .
$$

By induction on $s=j-i$, we have

$$
l_{\mathbf{u}_{i, j}}=x_{i} x_{i+1} \ldots x_{j} .
$$

This is because when $s=0$ we have $i=j$, and the unique possibility is $l_{\mathbf{u}_{i, i}}=x_{i}$. If we remove the last letter (when $j-i>0$ ), we must obtain a Lyndon word, so the last letter must be $x_{j}$.

Type $B_{\theta}$ : For convenience, we use the following vertex numbering:

$$
\circ^{1} \Longleftarrow \circ^{2}-\circ^{3} \ldots \quad \circ^{\theta-1}-\circ^{\theta} .
$$

The roots are of the form $\mathbf{u}_{i, j}:=\sum_{k=i}^{j} \alpha_{k}$, or

$$
\mathbf{v}_{i, j}:=2 \sum_{k=1}^{i} \alpha_{k}+\sum_{k=i+1}^{j} \alpha_{k} .
$$

In the first case we have $l_{\mathbf{u}_{i, j}}=x_{i} x_{i+1} \ldots x_{j}$, as above. In the second case, if $j=i+1$, we must have $x_{i+1}$ as the last letter to obtain a decomposition in two words $x_{1} \cdots x_{i}$; if $j>i+1$, the last letter must be $x_{j}$, so we obtain

$$
l_{\mathbf{v}_{i, j}}=x_{1} x_{2} \ldots x_{i} x_{1} x_{2} \ldots x_{j} .
$$

$\underline{\text { Type } C_{\theta}}$ : The roots are of the form $\mathbf{u}_{i, j}:=\sum_{k=i}^{j} \alpha_{k}$, or

$$
\mathbf{w}_{i, j}:=\sum_{k=i}^{j-1} \alpha_{k}+2 \sum_{k=j}^{\theta-1} \alpha_{k}+\alpha_{\theta}, \quad i \leq j<\theta .
$$


As before, $l_{\mathbf{u}_{i, j}}=x_{i} x_{i+1} \ldots x_{j}$. Now, if $i<j$, the least letter $x_{i}$ has degree 1 , so if we remove the last letter, we obtain a Lyndon word; that is, $\mathbf{w}_{i, j}-x_{s}$ is a root, and then $x_{s}=x_{j}$, so

$$
l_{\mathbf{w}_{i, j}}=x_{i} x_{i+1} \ldots x_{\theta-1} x_{\theta} x_{\theta-1} \ldots x_{j} .
$$

When $i=j, a_{i}=2$, so there are one or two Lyndon words $\beta_{j}$ as before. Since $\mathbf{w}-x_{s}$ is not a root, for $s=i+1, \ldots, \theta$, and $i<s$, there are two Lyndon words $\beta_{1} \geq \beta_{2}$, and $\beta_{1}+\beta_{2}=2 \sum_{k=i}^{\theta-1} \alpha_{k}$. The only possibility is $\beta_{1}=\beta_{2}=x_{i} x_{i+1} \ldots x_{\theta-1}$; that is,

$$
l_{\mathbf{w}_{i, i}}=x_{i} x_{i+1} \ldots x_{\theta-1} x_{i} x_{i+1} \ldots x_{\theta-1} x_{\theta} .
$$

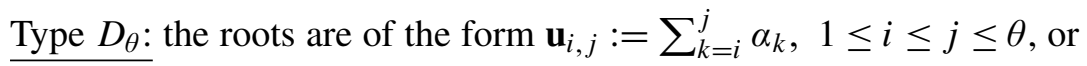

$$
\begin{aligned}
\mathbf{z}_{i, j} & :=\sum_{k=i}^{j-1} \alpha_{k}+2 \sum_{k=j}^{\theta-2} \alpha_{k}+\alpha_{\theta-1}+\alpha_{\theta}, i<j \leq \theta-2, \\
\overline{\mathbf{z}}_{i} & :=\sum_{k=i}^{\theta-2} \alpha_{k}+\alpha_{\theta}, \quad 1 \leq i \leq \theta-2 .
\end{aligned}
$$

As above, $l_{\mathbf{u}_{i, j}}=x_{i} x_{i+1} \ldots x_{j}$ if $j \leq n-1$. When the roots are of type $\overline{\mathbf{z}}_{i}$, we have $s=\theta$, since $\overline{\mathbf{z}}_{i}-x_{s}$ must be a root (if $x_{s}$ is the last letter); thus $l_{\overline{\mathbf{z}}_{i}}=x_{i} x_{i+1} \ldots x_{\theta-2} x_{\theta}$ is the unique possibility.

Now, when $\alpha=\mathbf{u}_{i, \theta}$, the last letter is $x_{\theta-1}$ or $x_{\theta}$ : if it is $x_{\theta}$, we have $l_{\mathbf{u}_{i, \theta}}=$ $x_{i} x_{i+1} \ldots x_{\theta-1} x_{\theta}$. Since $m_{\theta-1, \theta}=0$, we have $x_{\theta-1} x_{\theta}=q_{\theta-1, \theta} x_{\theta} x_{\theta-1}$, so

$$
x_{i} x_{i+1} \ldots x_{\theta-1} x_{\theta} \equiv x_{i} x_{i+1} \ldots x_{\theta-2} x_{\theta} x_{\theta-1} \bmod I,
$$

and then $x_{i} x_{i+1} \ldots x_{\theta-1} x_{\theta} \notin S_{I}$. So, $l_{\mathbf{u}_{i, \theta}}=x_{i} \ldots x_{\theta-2} x_{\theta} x_{\theta-1}$.

In the last case, note that if $j=n-2$, the unique possibility is $\beta_{t}$ as before, because the least letter $x_{i}$ has degree 1 and $x_{s}=x_{\theta-2}$ (since $\alpha-\alpha_{s}$ is a root). Hence $l_{\mathbf{z}_{i, \theta-2}}=x_{i} \ldots x_{\theta-2} x_{\theta} x_{\theta-1} x_{\theta-2}$, and inductively,

$$
l_{\mathbf{z}_{i, j}}=x_{i} \ldots x_{\theta-2} x_{\theta} x_{\theta-1} x_{\theta-2} \ldots x_{j} .
$$

Type $E_{6}$ : Let $\alpha=\sum_{j=1}^{6} a_{j} \alpha_{j}$. If $a_{6}=0, \alpha$ corresponds to the Dynkin subdiagram of type $D_{5}$ determined by $1,2,3,4,5$, and we obtain $l_{\alpha}$ as above. If $a_{1}=0$ then $\alpha$ corresponds to the Dynkin subdiagram of type $D_{5}$ determined by $2,3,4,5,6$; the numbering is different from the one given in (3-4). Anyway, the roots are defined in a similar way, and we obtain the same list as in [Lalonde and Ram 1995, Fig.1]. If $a_{4}=0$, then $\alpha$ corresponds to the Dynkin subdiagram of type $A_{5}$ determined by $1,2,3,5,6$.

So we restrict our attention to the case $a_{i} \neq 0, i=1,2,3,4,5,6$. We consider each case in turn: 
- $\alpha=\alpha_{1}+\alpha_{2}+\alpha_{3}+\alpha_{4}+\alpha_{5}+\alpha_{6}$ : since $a_{1}=1, \alpha-\alpha_{s}=\beta_{1}$ is a root, where $\alpha_{s}$ is the last letter. Then $s=2$ or $s=6$. In the second case, $l_{\beta_{1}}=x_{1} x_{2} x_{3} x_{4} x_{5}$, but using that $x_{2} x_{3}=q_{23} x_{3} x_{2}$, we have $x_{1} x_{2} x_{3} x_{4} x_{5} \notin S_{I}$. So $s=2$, and $l_{\alpha}=x_{1} x_{3} x_{4} x_{5} x_{6} x_{2}$.

- $\alpha=\alpha_{1}+\alpha_{2}+\alpha_{3}+2 \alpha_{4}+\alpha_{5}+\alpha_{6}$ : from $a_{1}=1$, we note that $\alpha-\alpha_{s}=\beta_{1}$ is a root. Then $s=4$, and $l_{\alpha}=x_{1} x_{3} x_{4} x_{5} x_{6} x_{2} x_{4}$.

- $\alpha=\alpha_{1}+\alpha_{2}+2 \alpha_{3}+2 \alpha_{4}+\alpha_{5}+\alpha_{6}$ : since $a_{1}=1, \alpha-\alpha_{s}=\beta_{1}$ is a root. So $s=3$, and $l_{\alpha}=x_{1} x_{3} x_{4} x_{5} x_{6} x_{2} x_{4} x_{3}$.

- $\alpha=\alpha_{1}+\alpha_{2}+\alpha_{3}+2 \alpha_{4}+2 \alpha_{5}+\alpha_{6}$ : since $a_{1}=1, \alpha-\alpha_{s}=\beta_{1}$ is a root. The only possibility is $s=5$, and $l_{\alpha}=x_{1} x_{3} x_{4} x_{5} x_{6} x_{2} x_{4} x_{5}$.

- $\alpha=\alpha_{1}+\alpha_{2}+2 \alpha_{3}+2 \alpha_{4}+2 \alpha_{5}+\alpha_{6}$ : as above $a_{1}=1$, and $\alpha-\alpha_{s}=\beta_{1}$ is a root. So $s=3$, and $l_{\alpha}=x_{1} x_{3} x_{4} x_{5} x_{6} x_{2} x_{4} x_{5} x_{3}$.

- $\alpha=\alpha_{1}+\alpha_{2}+2 \alpha_{3}+3 \alpha_{4}+2 \alpha_{5}+\alpha_{6}$ : since $a_{1}=1, \alpha-\alpha_{s}=\beta_{1}$ is a root. Then $s=4$ and $l_{\alpha}=x_{1} x_{3} x_{4} x_{5} x_{6} x_{2} x_{4} x_{5} x_{3} x_{4}$.

- $\alpha=\alpha_{1}+2 \alpha_{2}+2 \alpha_{3}+3 \alpha_{4}+2 \alpha_{5}+\alpha_{6}$ : since $a_{1}=1, \alpha-\alpha_{s}=\beta_{1}$ is a root. So $s=2$, and $l_{\alpha}=x_{1} x_{3} x_{4} x_{5} x_{6} x_{2} x_{4} x_{5} x_{3} x_{4}$.

Type $E_{7}$ : If $\alpha=\sum_{j=1}^{7} a_{j} \alpha_{j}$ and $a_{7}=0$, the root corresponds to the subdiagram of type $D_{6}$ determined by $1,2,3,4,5,6$, and we obtain $l_{\alpha}$ as above. If $a_{1}=0$, it corresponds to the subdiagram of type $E_{6}$ determined by $2,3,4,5,6,7$. If $a_{5}=0$, then $\alpha$ corresponds to the subdiagram of type $A_{6}$ determined by $1,2,3,4,6,7$.

As above, consider each case where $a_{i} \neq 0, i=1,2,3,4,5,6,7$ :

- $\alpha=\alpha_{1}+\alpha_{2}+\alpha_{3}+\alpha_{4}+\alpha_{5}+\alpha_{6}+\alpha_{7}$ : since $a_{1}=1, \alpha-\alpha_{s}=\beta_{1}$ is a root, if $\alpha_{s}$ is the last letter. Then $s=2$ or $s=7$. In the second case, $l_{\beta_{1}}=x_{1} x_{2} x_{3} x_{4} x_{5} x_{6}$, but from $x_{2} x_{3}=q_{23} x_{3} x_{2}$, we have $x_{1} x_{2} x_{3} x_{4} x_{5} x_{6} x_{7} \notin S_{I}$. So $s=2$, and $l_{\alpha}=$ $x_{1} x_{3} x_{4} x_{5} x_{6} x_{7} x_{2}$.

- $\alpha=\alpha_{1}+\alpha_{2}+\alpha_{3}+2 \alpha_{4}+\alpha_{5}+\alpha_{6}+\alpha_{7}$ : now $s=4,7$. We discard the case $s=7$ since $m_{47}=0$; for the case $s=4$ we have $l_{\alpha}=x_{1} x_{3} x_{4} x_{5} x_{6} x_{7} x_{2} x_{4}$.

- $\alpha=\alpha_{1}+\alpha_{2}+2 \alpha_{3}+2 \alpha_{4}+\alpha_{5}+\alpha_{6}+\alpha_{7}$ : as above, $s=3,7$, but we discard $s=7$ since $m_{37}=0$, so $l_{\alpha}=x_{1} x_{3} x_{4} x_{5} x_{6} x_{7} x_{2} x_{4} x_{3}$.

- $\alpha=\alpha_{1}+\alpha_{2}+\alpha_{3}+2 \alpha_{4}+2 \alpha_{5}+\alpha_{6}+\alpha_{7}$ : now $s=5,7$, and we discard the case $s=7$ because $m_{57}=0$, so $l_{\alpha}=x_{1} x_{3} x_{4} x_{5} x_{6} x_{7} x_{2} x_{4} x_{5}$.

- $\alpha=\alpha_{1}+\alpha_{2}+2 \alpha_{3}+2 \alpha_{4}+2 \alpha_{5}+\alpha_{6}+\alpha_{7}$ : now $s=3,7$, and as above we discard the case $s=7$, so $l_{\alpha}=x_{1} x_{3} x_{4} x_{5} x_{6} x_{7} x_{2} x_{4} x_{5} x_{3}$.

- $\alpha=\alpha_{1}+\alpha_{2}+2 \alpha_{3}+3 \alpha_{4}+2 \alpha_{5}+\alpha_{6}+\alpha_{7}$ : now $s=4$, and therefore we have $l_{\alpha}=x_{1} x_{3} x_{4} x_{5} x_{6} x_{7} x_{2} x_{4} x_{5} x_{3} x_{4}$.

- $\alpha=\alpha_{1}+2 \alpha_{2}+2 \alpha_{3}+3 \alpha_{4}+2 \alpha_{5}+\alpha_{6}+\alpha_{7}$ : now $s=2$, as above, and $l_{\alpha}=$ $x_{1} x_{3} x_{4} x_{5} x_{6} x_{7} x_{2} x_{4} x_{5} x_{3} x_{4} x_{2}$. 
- $\alpha=\alpha_{1}+\alpha_{2}+\alpha_{3}+2 \alpha_{4}+2 \alpha_{5}+2 \alpha_{6}+\alpha_{7}$ : as above, the unique possibility is $s=6$, so $l_{\alpha}=x_{1} x_{3} x_{4} x_{5} x_{6} x_{7} x_{2} x_{4} x_{5} x_{6}$.

- $\alpha=\alpha_{1}+\alpha_{2}+2 \alpha_{3}+2 \alpha_{4}+2 \alpha_{5}+2 \alpha_{6}+\alpha_{7}: s=3, l_{\alpha}=x_{1} x_{3} x_{4} x_{5} x_{6} x_{7} x_{2} x_{4} x_{5} x_{6} x_{3}$.

- $\alpha=\alpha_{1}+\alpha_{2}+2 \alpha_{3}+3 \alpha_{4}+2 \alpha_{5}+2 \alpha_{6}+\alpha_{7}: s=4, l_{\alpha}=x_{1} x_{3} x_{4} x_{5} x_{6} x_{7} x_{2} x_{4} x_{5} x_{6} x_{3} x_{4}$.

- $\alpha=\alpha_{1}+2 \alpha_{2}+2 \alpha_{3}+3 \alpha_{4}+2 \alpha_{5}+2 \alpha_{6}+\alpha_{7}: s=2$, and in this case we obtain $l_{\alpha}=x_{1} x_{3} x_{4} x_{5} x_{6} x_{7} x_{2} x_{4} x_{5} x_{6} x_{3} x_{4} x_{2}$.

- $\alpha=\alpha_{1}+\alpha_{2}+2 \alpha_{3}+3 \alpha_{4}+3 \alpha_{5}+2 \alpha_{6}+\alpha_{7}: s=5$, and in this case we obtain $l_{\alpha}=x_{1} x_{3} x_{4} x_{5} x_{6} x_{7} x_{2} x_{4} x_{5} x_{6} x_{3} x_{4} x_{5}$.

- $\alpha=\alpha_{1}+2 \alpha_{2}+2 \alpha_{3}+3 \alpha_{4}+3 \alpha_{5}+2 \alpha_{6}+\alpha_{7}$ : as above, $s=2$, and we get $l_{\alpha}=x_{1} x_{3} x_{4} x_{5} x_{6} x_{7} x_{2} x_{4} x_{5} x_{6} x_{3} x_{4} x_{5} x_{2}$.

- $\alpha=\alpha_{1}+2 \alpha_{2}+2 \alpha_{3}+4 \alpha_{4}+3 \alpha_{5}+2 \alpha_{6}+\alpha_{7}: s=4$, and in this case we obtain $l_{\alpha}=x_{1} x_{3} x_{4} x_{5} x_{6} x_{7} x_{2} x_{4} x_{5} x_{6} x_{3} x_{4} x_{5} x_{2} x_{4}$.

- $\alpha=\alpha_{1}+2 \alpha_{2}+3 \alpha_{3}+4 \alpha_{4}+3 \alpha_{5}+2 \alpha_{6}+\alpha_{7}: s=3$, and in this case we obtain $l_{\alpha}=x_{1} x_{3} x_{4} x_{5} x_{6} x_{7} x_{2} x_{4} x_{5} x_{6} x_{3} x_{4} x_{5} x_{2} x_{4} x_{3}$.

- $\alpha=2 \alpha_{1}+2 \alpha_{2}+3 \alpha_{3}+4 \alpha_{4}+3 \alpha_{5}+2 \alpha_{6}+\alpha_{7}$ : now there are one or two words $\beta_{j}$. Since $\alpha-\alpha_{s} \in \Delta^{+}$if and only if $s=1$ and $x_{1}$ is not the last letter (because it is the least letter), there are two words $\beta_{j}$. So looking at the roots we obtain $s=7$, and $l_{\alpha}=\left(x_{1} x_{3} x_{4} x_{5} x_{6} x_{2} x_{4} x_{5} x_{3} x_{4} x_{2}\right)\left(x_{1} x_{3} x_{4} x_{5} x_{6}\right) x_{7}$

Type $E_{8}$ : Consider $\alpha=\sum_{j=1}^{8} a_{j} \alpha_{j}$; if $a_{8}=0$, the root corresponds to the subdiagram of type $D_{7}$ determined by $1,2,3,4,5,6,7$, and we obtain $l_{\alpha}$ as in that case. If $a_{1}=0$, it corresponds to the subdiagram of type $E_{7}$ determined by $2,3,4,5,6,7,8$. If $a_{6}=0$, then $\alpha$ corresponds to a subdiagram of type $A_{7}$ determined by $1,2,3,4,5,7,8$.

So, we consider the case $a_{i} \neq 0, i=1,2,3,4,5,6,7,8$, and solve it case by case in a similar way as for $E_{7}$, by induction on the height.

Type $F_{4}$ : Now $\alpha=\sum_{j=1}^{4} a_{j} \alpha_{j}$. If $a_{4}=0$, then it corresponds to the subdiagram of type $B_{3}$ determined by $1,2,3$, so we obtain $l_{\alpha}$ as before. If $a_{1}=0, \alpha$ corresponds to the subdiagram of type $C_{3}$ determined by $2,3,4$.

So consider the case $a_{i} \neq 0, i=1,2,3,4$ :

- $\alpha=\alpha_{1}+\alpha_{2}+\alpha_{3}+\alpha_{4}: a_{1}=1$, so $\alpha-\alpha_{s}=\beta_{1}$ is a root, where $\alpha_{s}$ is the last letter. Then $s=4$, and $l_{\alpha}=x_{1} x_{2} x_{3} x_{4}$.

- $\alpha=\alpha_{1}+\alpha_{2}+2 \alpha_{3}+\alpha_{4}: a_{1}=1$, so $\alpha-\alpha_{s}=\beta_{1}$ is a root. Now $s=3$ or $s=4$. If $s=4$, then $l_{\alpha}=x_{1} x_{2} x_{3}^{2} x_{4}$. But $m_{34}=2$, so

$$
x_{3}^{2} x_{4} \equiv q_{34}\left(1+q_{33}\right) x_{3} x_{4} x_{3}-q_{33} q_{34} x_{4} x_{3}^{2} \bmod I,
$$

and $x_{1} x_{2} x_{3}^{2} x_{4} \notin S_{I}$, a contradiction. So $s=3$, and we have $l_{\alpha}=x_{1} x_{2} x_{3} x_{4} x_{3}$. 
- $\alpha=\alpha_{1}+2 \alpha_{2}+2 \alpha_{3}+\alpha_{4}: a_{1}=1$, and as above, $s=2$ or $s=4:$ if $s=4$, then $l_{\alpha}=x_{1} x_{2} x_{3}^{2} x_{2} x_{4}$, but it is not an element of $S_{I}$, because $x_{2} x_{4} \equiv q_{24} x_{2} x_{4} \bmod I$. Then $s=2$, and $l_{\alpha}=x_{1} x_{2} x_{3} x_{4} x_{3} x_{2}$.

- $\alpha=\alpha_{1}+2 \alpha_{2}+3 \alpha_{3}+\alpha_{4}: a_{1}=1$, so $s=3$, and we have $l_{\alpha}=x_{1} x_{2} x_{3} x_{4} x_{3} x_{2} x_{3}$.

- $\alpha=\alpha_{1}+\alpha_{2}+2 \alpha_{3}+2 \alpha_{4}: a_{1}=1$, so $s=4$, and $l_{\alpha}=x_{1} x_{2} x_{3} x_{4} x_{3} x_{4}$.

- $\alpha=\alpha_{1}+2 \alpha_{2}+2 \alpha_{3}+2 \alpha_{4}: a_{1}=1$, so $s=2$ or $s=4$, but we discard the case $s=4$ since $x_{2} x_{4} \equiv q_{24} x_{2} x_{4} \bmod I$. So, $l_{\alpha}=x_{1} x_{2} x_{3} x_{4} x_{3} x_{4} x_{2}$.

- $\alpha=\alpha_{1}+2 \alpha_{2}+3 \alpha_{3}+2 \alpha_{4}: a_{1}=1$, so $s=3$, and $l_{\alpha}=x_{1} x_{2} x_{3} x_{4} x_{3} x_{4} x_{2} x_{3}$.

- $\alpha=\alpha_{1}+2 \alpha_{2}+4 \alpha_{3}+2 \alpha_{4}: a_{1}=1$, so $s=3$, and $l_{\alpha}=x_{1} x_{2} x_{3} x_{4} x_{3} x_{4} x_{2} x_{3}^{2}$.

- $\alpha=\alpha_{1}+3 \alpha_{2}+4 \alpha_{3}+2 \alpha_{4}: a_{1}=1$, so $s=2$, and $l_{\alpha}=x_{1} x_{2} x_{3} x_{4} x_{3} x_{4} x_{2} x_{3}^{2} x_{2}$.

- $\alpha=2 \alpha_{1}+3 \alpha_{2}+4 \alpha_{3}+2 \alpha_{4}: a_{1}=2$, and there are one or two Lyndon words $\beta_{j}$. If there is only one, $\beta_{1}=\alpha-\alpha_{s} \in \Delta^{+}$. The only possibility is $s=1$, but it contradicts that $l_{\alpha}$ is a Lyndon word. Hence there exist $\beta_{1}, \beta_{2} \in \Delta^{+}$such that $\beta_{1}+\beta_{2}=\alpha-\alpha_{s}$, and $\beta_{2}$ is a beginning of $\beta_{1}$. So $s=2$ and $\beta_{1}=\beta_{2}=\alpha_{1}+\alpha_{2}+2 \alpha_{3}+\alpha_{4}$, i.e., $l_{\alpha}=x_{1} x_{2} x_{3} x_{4} x_{3} x_{1} x_{2} x_{3} x_{4} x_{3} x_{2}$.

Type $G_{2}$ : the roots are $\alpha_{1}, \alpha_{2}, \alpha_{1}+\alpha_{2}, 2 \alpha_{1}+\alpha_{2}, 3 \alpha_{1}+\alpha_{2}, 3 \alpha_{1}+2 \alpha_{2}$ :

$$
l_{\alpha_{1}}=x_{1}, \quad l_{\alpha_{2}}=x_{2}, \quad l_{m \alpha_{1}+\alpha_{2}}=x_{1}^{m} x_{2}, \quad m=1,2,3 .
$$

If $\alpha=3 \alpha_{1}+2 \alpha_{2}$, the last letter is $x_{2}$. If we suppose $\beta_{1}=3 \alpha_{1}+\alpha_{2}$, then $l_{\alpha}=x_{1}^{3} x_{2}^{2}$, but

$$
\left(\operatorname{ad} x_{2}\right)^{2} x_{1}=x_{2}^{2} x_{1}-q_{21}\left(1+q_{22}\right) x_{2} x_{1} x_{2}+q_{22} q_{21} x_{1} x_{2}^{2} \equiv 0 \quad \bmod I,
$$

so we have

$$
x_{1}^{3} x_{2}^{2} \equiv\left(q_{22}^{-1}+1\right) x_{1}^{2} x_{2} x_{1} x_{2}-q_{22}^{-1} q_{21}^{-1} x_{1}^{2} x_{2}^{2} x_{1} \quad \bmod I,
$$

and then $l_{\alpha}=x_{1}^{3} x_{2}^{2} \notin S_{I}$ because $q_{22}^{-1} q_{21}^{-1} \neq 0$, so there are at least two words $\beta_{j}$. Analogously, if we suppose that there are three words $\beta_{j}$, we obtain $l_{\beta_{1}}=l_{\beta_{2}}=$ $x_{1}>l_{\beta_{3}}=x_{1} x_{2}$ since $\beta_{1} \geq \beta_{2} \geq \beta_{3}$ and $\beta_{1}+\beta_{2}+\beta_{3}=3 \alpha_{1}+\alpha_{2}$; moreover $l_{\alpha}=x_{1}^{3} x_{2}^{2} \notin S_{I}$. So there are two Lyndon words of degree $\beta_{1} \geq \beta_{2}$, and the unique possibility is $\beta_{1}=2 \alpha_{1}+\alpha_{2}, \beta_{2}=\alpha_{1}$; that is, $l_{\alpha}=x_{1}^{2} x_{2} x_{1} x_{2}$.

4C. Dimensions of Nichols algebras of standard braidings. We begin with the standard braidings of types $C_{\theta}, D_{\theta}, E_{6}, E_{7}, E_{8}, F_{4}$, which are of Cartan type.

Proposition 4.4. Let $V$ a braided vector space of Cartan type, with $q_{44} \in \mathbb{G}_{N}$ if $V$ is of type $F_{4}$ and $q_{11} \in \mathbb{G}_{N}$ otherwise, in each case for some $N \in \mathbb{N}$. The dimension of the associated Nichols algebra $\mathfrak{B}(V)$ is as follows:

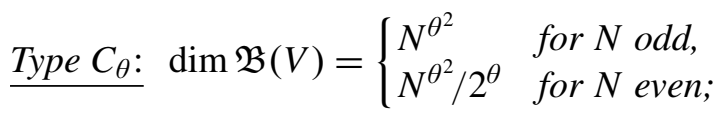


Type $F_{4}: \operatorname{dim} \mathfrak{B}(V)= \begin{cases}N^{24} & \text { for } N \text { odd }, \\ N^{24} / 2^{12} & \text { for } N \text { even; }\end{cases}$

Types $D_{\theta}, E_{6}, E_{7}, E_{8}$ : $\operatorname{dim} \mathfrak{B}(V)=N^{\left|\Delta^{+}\right|}$.

The last case corresponds to simply laced Dynkin diagrams.

Proof. If $N$ is odd, then ord $q^{2}=\operatorname{ord} q=N$, but if $N$ is even, we have ord $q^{2}=N / 2$. Since the braiding is of Cartan type,

$$
q_{s_{i}(\alpha)}=\chi\left(s_{i}(\alpha), s_{i}(\alpha)\right)=\tilde{\chi}(\alpha, \alpha)=\chi(\alpha, \alpha)=q_{\alpha} .
$$

Using this, we just have to determine how many roots there are in the orbit of each simply root.

When $V$ is of type $C_{\theta}$, we have $q_{i i}=q$, except for $q_{\theta \theta}=q^{2}$. The roots in the orbit of $\alpha_{\theta}$ by the action of the Weyl group are $q_{\mathbf{w}_{i i}}$ for $1 \leq i<\theta$, and the others are in the orbit of $\alpha_{j}$, for some $j<\theta$. Hence there are $\theta$ roots such that $q_{\alpha}=q^{2}$, and $q_{\alpha}=q$ for the rest.

When $V$ is of type $F_{4}$, we have $q_{11}=q_{22}=q^{2}$ and $q_{33}=q_{44}=q$. There are exactly 12 roots in the union of orbits corresponding to $\alpha_{1}$ and $\alpha_{2}$, and the other 12 are in the union of orbits corresponding to $\alpha_{3}$ and $\alpha_{4}$. So

$$
\left|\left\{\alpha \in \Delta^{+}: q_{\alpha}=q\right\}\right|=\left|\left\{\alpha \in \Delta^{+}: q_{\alpha}=q^{2}\right\}\right|=12 .
$$

When $V$ is of type $D$ or $E$, all the $q_{\alpha}$ equal $q$ because $q_{i i}=q$, for all $1 \leq i \leq \theta$.

The formula for the dimension follows from Theorem 2.6(ii) and Corollary 4.2.

Now we treat the types $A_{\theta}, B_{\theta}$ and $G_{2}$.

Proposition 4.5. Let $V$ be a standard braided vector space of type $A_{\theta}$ as in Proposition 3.9. The associated Nichols algebra $\mathfrak{B}(V)$ is of finite dimension if and only if $q$ is a root of unity of order $N \geq 2$. In this case,

$$
\operatorname{dim} \mathfrak{B}(V)=2^{\left(\begin{array}{c}
\theta+1 \\
2
\end{array}\right)-\left(\begin{array}{c}
t \\
2
\end{array}\right)-\left(\begin{array}{c}
\theta+1-t \\
2
\end{array}\right)} N^{\left(\begin{array}{l}
t \\
2
\end{array}\right)+\left(\begin{array}{c}
\theta+1-t \\
2
\end{array}\right)}
$$

where $t=\theta+1-\sum_{k=1}^{j}(-1)^{j-k} i_{k}$.

Proof. First, $q$ is a root of unity of order $N \geq 2$ because the height of each PBW generator is finite. To calculate the dimension, recall that from Corollary 4.2, we have to determine $q_{\alpha}$ for $\alpha \in \Delta_{C}$. As before, $\mathbf{u}_{i j}=\sum_{k=i}^{j} \mathbf{e}_{k}, i \leq j$, and we have

$$
\Delta(\mathfrak{B}(V))=\left\{\mathbf{u}_{i j}: 1 \leq i \leq j \leq \theta\right\} .
$$

If $1 \leq i \leq j \leq \theta$, we define

$$
\kappa_{i j}:=\operatorname{card}\left\{k \in\{i, \ldots, j\}: q_{k k}=-1\right\} .
$$


We prove by induction on $j-i$ that

- if $\kappa_{i j}$ is odd, then $q_{\mathbf{u}_{i j}}=-1$;

- if $\kappa_{i j}$ is even, then $q_{\mathbf{u}_{i j}}=q_{j, j+1}^{-1} q_{j+1, j}^{-1}$.

Here $q_{\theta, \theta+1} q_{\theta+1, \theta}=q_{\theta \theta}^{-2} q_{\theta, \theta-1}^{-1} q_{\theta-1, \theta}^{-1}$.

If $j-i=0$, then $q_{\mathbf{u}_{i i}}=q_{i i}$; in this case, $\kappa_{i i}=1$ if $q_{i i}=-1$ or $\kappa_{i i}=0$ if $q_{i i}=\left(q_{i, i+1} q_{i+1, i}\right)^{-1} \neq-1$. Now assume this is valid for a certain $j$, and calculate it for $j+1$ :

$$
\begin{aligned}
q_{\mathbf{u}_{i, j+1}}= & \chi\left(\mathbf{u}_{i j}+\mathbf{e}_{j+1}, \mathbf{u}_{i j}+\mathbf{e}_{j+1}\right)=q_{\mathbf{u}_{i j}} \chi\left(\mathbf{u}_{i j}, \mathbf{e}_{j+1}\right) \chi\left(\mathbf{e}_{j+1}, \mathbf{u}_{i j}\right) q_{j+1, j+1} \\
= & q_{\mathbf{u}_{i j}} q_{j, j+1} q_{j+1, j} q_{j+1, j+1} \\
& = \begin{cases}q_{\mathbf{u}_{i j}} & \text { if } q_{j+1, j+1} \neq-1\left(\kappa_{i, j+1}=\kappa_{i j}\right), \\
(-1) q q^{-1}=-1 & \text { if } q_{j+1, j+1}=-1, \kappa_{i j} \text { even, } \\
(-1) q(-1)=q & \text { if } q_{j+1, j+1}=-1, \kappa_{i j} \text { odd } .\end{cases}
\end{aligned}
$$

This proves the inductive step; to calculate the dimension of $\mathfrak{B}(V)$ we have to calculate the number of $\mathbf{u}_{i j}$ such that

$$
q_{\mathbf{u}_{i j}}=q_{i, i+1}^{-1} q_{i+1, i}^{-1}=q^{ \pm 1}
$$

that is, $\operatorname{card}\left\{\kappa_{i j}: i \leq j, \kappa_{i j}\right.$ even $\}$.

We consider an $1 \times(\theta+1)$ board, numbered from 1 to $\theta+1$, and recursively paint its squares white or black: square $\theta+1$ is white, and square $i$ has the same color as square $i+1$ if and only if $q_{i i} \neq-1$. The possible colorings of this board are in bijective correspondence with the choices of $1 \leq i_{1}<\cdots<i_{j} \leq \theta$ for all $j$ (the positions where we put a -1 in the corresponding $q_{i i}$ of the braiding), and the number of white squares is

$$
t=1+\left(\theta-i_{j}\right)+\left(i_{j-1}-i_{j-2}\right)+\cdots=\theta+1-\sum_{k=1}^{j}(-1)^{j-k} i_{k}
$$

Thus $\operatorname{card}\left\{\kappa_{i j}: i \leq j, \kappa_{i j}\right.$ even $\}$ is the number of pairs $(a, b)$ (where $a=i$ and $b=j+1$ ) such that $1 \leq a<b \leq \theta+1$ and the squares in positions $a$ and $b$ are of the same color; this number is $\left(\begin{array}{l}t \\ 2\end{array}\right)+\left(\begin{array}{c}\theta+1-t \\ 2\end{array}\right)$. This yields (4-2).

Proposition 4.6. Let $V$ be a standard braided vector space of type $B_{\theta}$ as in Proposition 3.10. If the braiding is as in cases (a) or (b) of that proposition, the associated Nichols algebra $\mathfrak{B}(V)$ has finite dimension if and only if $q$ is a root of unity of order $N \geq 2$ in case (a), or $N>2$ in case (b).

When finite, the dimension of $\mathfrak{B}(V)$ is as follows, where $t=\theta-\sum_{k=1}^{j}(-1)^{j-k} i_{k}$ : 
- If the braiding is as in (a) of Proposition 3.10,

$$
\operatorname{dim} \mathfrak{B}(V)= \begin{cases}3^{3} N^{2} & \text { if } 3 \text { does not divide } N, \\ 3^{2} N^{2} & \text { if } 3 \text { divides both } N \text { and } \operatorname{ord}\left(\zeta^{-1} q\right), \\ 3 N^{2} & \text { if } 3 \text { divides } N \text { but not } \operatorname{ord}\left(\zeta^{-1} q\right) .\end{cases}
$$

- If the braiding is as in (b), then $0 \leq j \leq d-1$ and

$$
\operatorname{dim} \mathfrak{B}(V)= \begin{cases}2^{2 t(\theta-t)+\theta} k^{\theta^{2}-2 t \theta+2 t^{2}} & \text { if } N=2 k, \\ 2^{(2 t+1)(\theta-t)+1} N^{\theta^{2}-2 t \theta+2 t^{2}} & \text { if } N \text { is odd } .\end{cases}
$$

- If the braiding is as in (c), then

$$
\operatorname{dim} \mathfrak{B}(V)=2^{\theta(\theta-1)} 3^{\theta^{2}-2 t \theta+2 t^{2}} .
$$

Proof. It is clear that $q$ should be a root of unity if $\operatorname{dim} \mathfrak{B}(V)$ is finite.

We now calculate $\operatorname{dim} \mathfrak{B}(V)$. From Corollary 4.2, we have to determine the $q_{\alpha}$ for $\alpha \in \Delta_{C}$, and multiply their orders. As before, $\mathbf{u}_{i j}=\sum_{k=i}^{j} \mathbf{e}_{k}$ for $1 \leq i \leq j \leq \theta$ and $\mathbf{v}_{i j}=2 \sum_{k=1}^{i} e_{k}+\sum_{k=i+1}^{j} e_{k}=2 e_{1, i}+e_{i+1, j}$ for $1 \leq i<j$; hence

$$
\Delta(\mathfrak{B}(V))=\left\{\mathbf{u}_{i j}: 1 \leq i \leq j \leq \theta\right\} \cup\left\{\mathbf{v}_{i j}: 1 \leq i<j \leq \theta\right\} .
$$

We calculate $q_{\mathbf{u}_{i j}}, 1<i \leq j \leq \theta$ as above, because they correspond to a braiding of standard $A_{\theta-1}$ type. We also calculate

$$
\begin{aligned}
q_{\mathbf{v}_{i j}} & =\chi\left(\mathbf{v}_{i j}, \mathbf{v}_{i j}\right)=\chi\left(\mathbf{u}_{1 i}, \mathbf{u}_{1 i}\right)^{4} \chi\left(\mathbf{u}_{1 i}, \mathbf{u}_{i+1, j}\right)^{2} \chi\left(\mathbf{u}_{i+1, j}, \mathbf{u}_{1 i}\right)^{2} q_{\mathbf{u}_{i+1, j}} \\
& =q_{11}^{4} q_{12}^{2} q_{21}^{2}\left(\prod_{k=2}^{i} q_{k k}^{2} q_{k-1, k} q_{k-1, k} q_{k+1, k} q_{k+1, k}\right)^{2} q_{\mathbf{u}_{i+1, j}}=q_{\mathbf{u}_{i+1, j}},
\end{aligned}
$$

where we have used the equalities $q_{i j} q_{j i}=1$ if $|i-j|>1, q_{11}^{4} q_{12}^{2} q_{21}^{2}=1$, and $q_{k k}^{2} q_{k-1, k} q_{k-1, k} q_{k+1, k} q_{k+1, k}=1$ if $2 \leq k \leq \theta-1$. To calculate the other $q_{\alpha}$ 's, we analyze each case:

(a) Note that $q_{\mathbf{e}_{1}}=\zeta, q_{\mathbf{e}_{1}+\mathbf{e}_{2}}=\zeta, q_{2 \mathbf{e}_{1}+\mathbf{e}_{2}}=\zeta q^{-1}, q_{\mathbf{e}_{2}}=q$. Setting $N^{\prime}=\operatorname{ord}\left(\zeta^{-1} q\right)$, we have $N^{\prime}=3 N$ if 3 does not divide $N ; N^{\prime}=N$ if 3 divides both $N$ and $N^{\prime}$; and $N^{\prime}=N / 3$ if 3 divides $N$ but not $N^{\prime}$ ( since $q=\zeta \rho$, with $\rho \in \mathbb{G}_{N^{\prime}}$ ).

(b) We have $q_{\mathbf{u}_{1 k}}=q^{-1} q_{\mathbf{u}_{2 k}}$. This equals $q^{2} q^{-1}=q$ if $\kappa_{2 k}$ is even, and $-q^{-1}$ if $\kappa_{2 k}$ is odd; moreover $q_{11}=q$. Also, $\kappa_{2 k}$ is even if and only if $j \in\left\{i_{j}+1, \theta\right\}$, or $i \in\left\{i_{j-2}+1, i_{j-1}\right\}$, and so on. Then, with

$$
t=\left(\theta-i_{j}\right)+\left(i_{j-1}-i_{j-2}\right)+\cdots=\theta-\sum_{k=1}^{j}(-1)^{j-k} i_{k}
$$

as in the statement of the proposition, there are $t$ numbers such that $\kappa_{i, \theta-1}$ is even. There are $2\left(\left(\begin{array}{c}t \\ 2\end{array}\right)+\left(\begin{array}{c}\theta-t \\ 2\end{array}\right)\right)$ roots such that $q_{\alpha}=q^{2}, 2\left(\left(\begin{array}{c}\theta \\ 2\end{array}\right)-\left(\begin{array}{c}t \\ 2\end{array}\right)-\left(\begin{array}{c}\theta-t \\ 2\end{array}\right)\right)$ roots such 
that $q_{\alpha}=-1, t+1$ roots such that $q_{\alpha}=q$ and $\theta-1-t$ roots such that $q_{\alpha}=-q^{-1}$. If $N=2 k$, then ord $\left(-q^{-1}\right)=2 k$ and $\operatorname{ord}\left(q^{2}\right)=k$, so

$$
\begin{aligned}
\operatorname{dim} \mathfrak{B}(V) & =2^{(\theta-1) \theta-t(t-1)-(\theta-t)(\theta-t-1)} k^{t(t-1)+(\theta-t)(\theta-t-1)}(2 k)^{\theta} \\
& =2^{2 t(\theta-t)+\theta} k^{\theta^{2}-2 t \theta+2 t^{2}} .
\end{aligned}
$$

If $N$ is odd, then ord $\left(-q^{-1}\right)=2 N$ and $\operatorname{ord}\left(q^{2}\right)=N$, so

$$
\begin{aligned}
\operatorname{dim} \mathfrak{B}(V)= & 2^{\theta(\theta-1)-t(t-1)-(\theta-t)(\theta-1-t)} N^{t(t-1)+(\theta-t)(\theta-1-t)+t+1} \\
& (2 N)^{\theta-1-t}=2^{(2 t+1)(\theta-t)+1} N^{\theta^{2}-2 t \theta+2 t^{2}} .
\end{aligned}
$$

(c) In a similar way, we have $q_{\mathbf{u}_{1 i}}=\left(-\zeta^{2}\right) q_{\mathbf{u}_{2 i}}$, which equals $\left(-\zeta^{2}\right)^{2}=\zeta$ if $\kappa_{2 i}$ is even, and $(-1)\left(-\zeta^{2}\right)=\zeta^{2}$ if $\kappa_{2 i}$ is odd; moreover $q_{11}=\zeta$. There are $2\left(\left(\begin{array}{c}t \\ 2\end{array}\right)+\left(\begin{array}{c}\theta-t \\ 2\end{array}\right)\right)$ roots such that $q_{\alpha}=-\zeta^{2}, 2\left(\left(\begin{array}{c}\theta \\ 2\end{array}\right)-\left(\begin{array}{c}t \\ 2\end{array}\right)-\left(\begin{array}{c}\theta-t \\ 2\end{array}\right)\right)$ roots such that $q_{\alpha}=-1, t+1$ roots such that $q_{\alpha}=\zeta$ and $\theta-1-t$ roots such that $q_{\alpha}=\zeta^{2}$. Since ord $\zeta=$ ord $\zeta^{2}=3$ and ord $\left(-\zeta^{2}\right)=6$, we have

$$
\begin{aligned}
\operatorname{dim} \mathfrak{B}(V) & =2^{\theta(\theta-1)-t(t-1)-(\theta-t)(\theta-1-t)} 6^{t(t-1)+(\theta-t)(\theta-1-t)} 3^{\theta} \\
& =2^{\theta(\theta-1)} 3^{\theta^{2}-2 t \theta+2 t^{2}} .
\end{aligned}
$$

Proposition 4.7. Let $V$ be a standard braided vector space of type $G_{2}$ as in Proposition 3.11. If the braiding is as in case (a) of that proposition, the associated Nichols algebra $\mathfrak{B}(V)$ is of finite dimension if and only if $q$ is a root of unity of order $N \geq 4$.

When finite, the dimension of $\mathfrak{B}(V)$ is as follows:

- In case (a) of Proposition 3.11,

$$
\operatorname{dim} \mathfrak{B}(V)= \begin{cases}N^{6} & \text { if } 3 \text { does not divide } N, \\ N^{6} / 27 & \text { if } 3 \text { divides } N\end{cases}
$$

\begin{tabular}{|c|c|c|c|c|c|c|c|}
\hline$\zeta^{2} \zeta^{\zeta^{-1}}{ }^{-1}$ & 8 & 4 & 2 & 8 & 2 & 4 & $2^{12}$ \\
\hline$\zeta^{2} \zeta^{3}-1$ & 2 & 8 & 2 & 4 & 8 & 4 & $2^{12}$ \\
\hline$\zeta^{\zeta} \zeta^{5}-1$ & 2 & 4 & 8 & 4 & 2 & 8 & $2^{12}$ \\
\hline
\end{tabular}

- In case (b), $\operatorname{dim} \mathfrak{B}(V)=2^{12}$.

Proof. For (a) note that $q$ is a root of unity because $x_{1}$ has finite height, and $q_{\alpha}=q$ if $\alpha \in\left\{e_{1}, e_{1}+e_{2}, 2 e_{1}+e_{2}\right\}$, while $q_{\alpha}=q^{3}$ if $\alpha \in\left\{e_{2}, 3 e_{1}+e_{2}, 3 e_{1}+2 e_{2}\right\}$.

Case (b) can be checked as follows:

type $\quad q_{x_{2}} \quad q_{x_{1} x_{2}} \quad q_{x_{1}^{3} x_{2}^{2}} \quad q_{x_{1}^{2} x_{2}} \quad q_{x_{1}^{3} x_{2}} \quad q_{x_{1}} \quad \operatorname{dim} \mathfrak{B}(V)$

This completes the proof. 


\section{Presentation by generators and relations of Nichols algebras of standard braided vector spaces}

In this section we give presentations for the Nichols algebras of standard braided vector spaces. We start with some technical results about relations and PBW bases in Section 5A; also we calculate the coproduct of some hyperwords in $T(V)$. In Sections 5B, 5C and 5D we express the braided commutator of two PBW generators as a combination of elements of the PBW basis under some assumptions. Then we obtain the desired presentation with a proof similar to the ones in [Andruskiewitsch and Dăscălescu 2005] and [Andruskiewitsch and Schneider 2002b]. In Section 5E we solve the problem when the braiding is of Cartan type using the transformation in Section 2C.

For rank two, a set of (not necessarily minimal) relations is given in Theorem 4 of [Heckenberger 2007].

5A. Some general relations. Let $V$ be a standard braided vector space with connected Dynkin diagram and let $C$ be the corresponding Cartan matrix. In what follows we assume that $C$ is symmetrizable and of finite type. Let $x_{1}, \ldots, x_{\theta}$ be an ordered basis of $V$ and $\left\{x_{\alpha}: \alpha \in \Delta^{+}(\mathfrak{B}(V))\right\}$ a set of PBW generators as in the previous section. Here $x_{\alpha} \in \mathfrak{B}(V)$ is, by abuse of notation, the image by the canonical projection of $x_{\alpha} \in T(V)$, the hyperword corresponding to a Lyndon word $l_{\alpha}$. As before, we write

$$
q_{\alpha}:=\chi(\alpha, \alpha) \quad \text { and } \quad N_{\alpha}:=\operatorname{ord} q_{\alpha} \quad \text { for } \alpha \in \Delta^{+}(\mathfrak{B}(V)) .
$$

Each $x_{\alpha}$ is homogeneous and has the same degree as $l_{\alpha}$. Also,

$$
x_{\alpha} \in T(V)_{g_{\alpha}}^{\chi_{\alpha}},
$$

where $g_{\alpha}=g_{1}^{b_{1}} \ldots g_{\theta}^{b_{\theta}}$ and $\chi_{\alpha}=\chi_{1}^{b_{1}} \ldots \chi_{\theta}^{b_{\theta}}$ if $\alpha=b_{1} \mathbf{e}_{1}+\cdots+b_{\theta} \mathbf{e}_{\theta}$.

Proposition 5.1. If the matrix of the braiding is symmetric, the PBW basis is orthogonal with respect to the bilinear form in Proposition 1.4.

Proof. We must prove that $(u \mid v)=0$, where $u \neq v$ are ordered products of PBW generators (we also allow powers greater than the corresponding heights). We argue by induction on $k:=\max \{\ell(u), \ell(v)\}$. If $k=1$, then $v$ is some $x_{j}$ and $u$ is either 1 or $x_{i}$; since $\left(x_{i} \mid x_{j}\right)=\delta_{i j}$ for all $i, j \in\{1, \ldots, \theta\}$, the proposition holds in this case.

Suppose the statement is valid when the length of both words is less than $k$, and let $u, v \in B_{I(V)}$ be distinct hyperwords such that one (or both) has length $k$. If both are hyperletters, they have different degrees $\alpha \neq \beta \in \mathbb{Z}^{\theta}$, so $u=x_{\alpha}, v=x_{\beta}$, and $\left(x_{\alpha} \mid x_{\beta}\right)=0$, since the homogeneous components are orthogonal for $(\mid)$. 
Suppose that $u=x_{\alpha}$ and $v=x_{\beta_{1}}^{h_{1}} \ldots x_{\beta_{m}}^{h_{m}}$, for some $x_{\beta_{1}}>\cdots>x_{\beta_{m}}$. If $u$ and $v$ have different $\mathbb{Z}^{\theta}$-degrees, they are orthogonal. Hence we assume that $\alpha=\sum_{j=1}^{m} h_{m} \beta_{m}$. By [Bourbaki 1968, VI, Proposition 19], we can reorder the $\beta_{i}$ 's, using $h_{i}$ copies of $\beta_{i}$, in such a way that each partial sum is a root. By [Rosso 1999, Proposition 21], the order induced by the Lyndon words $l_{\alpha}$ is convex (the order on Lyndon words used there is the same as ours). Therefore $\beta_{m}<\alpha$. Using Lemma 1.9 and (1-8),

$$
(u \mid v)=\left(x_{\alpha} \mid w\right)\left(1 \mid x_{\beta_{m}}\right)+(1 \mid w)\left(x_{\alpha_{n}} \mid x_{\beta_{m}}\right)+\sum_{\substack{l_{1} \geq \cdots \geq l_{p}>\alpha \\ l_{i} \in L}}\left(x_{l_{1}, \ldots, l_{p}} \mid w\right)\left(\left[l_{1}\right]_{c} \cdots\left[l_{p}\right]_{c} \mid x_{\beta_{m}}\right),
$$

where $v=w x_{\beta_{n}}$. Note that $\left(1 \mid x_{\beta_{m}}\right)=(1 \mid w)=0$. Also, $\left[l_{1}\right]_{c} \cdots\left[l_{p}\right]_{c}$ is a linear combination of greater hyperwords of the same degree and an element of $I(V)$. From the inductive hypothesis and the fact that $I(V)$ is the radical of the bilinear form, we see that $\left(\left[l_{1}\right]_{c} \cdots\left[l_{p}\right]_{c} \mid x_{\beta_{m}}\right)=0$.

Now consider

$$
\begin{aligned}
& u=x_{\alpha_{1}}^{j_{1}} \ldots x_{\alpha_{n}}^{j_{n}} \text { with } x_{\alpha_{1}}>\ldots>x_{\alpha_{n}}, \\
& v=x_{\beta_{1}}^{h_{1}} \ldots x_{\beta_{m}}^{h_{m}} \text { with } x_{\beta_{1}}>\cdots>x_{\beta_{m}} .
\end{aligned}
$$

Since the bilinear form is symmetric, we may as well assume that $x_{\alpha_{n}} \leq x_{\beta_{m}}$. Using Lemma 1.9 and (1-8), we obtain

$$
\begin{aligned}
(u \mid v)=(w \mid 1)\left(x_{\alpha_{n}} \mid v\right)+\sum_{i=0}^{h_{m}}\left(\begin{array}{c}
h_{m} \\
i
\end{array}\right)_{q_{\beta_{m}}}\left(w \mid x_{\beta_{1}}^{h_{1}} \ldots x_{\beta_{m-1}}^{h_{m-1}} x_{\beta_{m}}^{i}\right)\left(x_{\alpha_{n}} \mid x_{\beta_{m}}^{h_{m}-i}\right) \\
+\sum_{\substack{l_{1} \geq \cdots \geq l_{p}>l, l_{i} \in L \\
0 \leq j \leq m}}\left(w \mid x_{l_{1}, \ldots, l_{p}}^{(j)}\right)\left(x_{\alpha_{n}} \mid\left[l_{1}\right]_{c} \ldots\left[l_{p}\right]_{c}\left[x_{\beta_{m}}\right]_{c}^{j}\right),
\end{aligned}
$$

where $w=x_{\alpha_{1}}^{h_{1}} \ldots x_{\alpha_{m}}^{h_{m}-1}$. Note that in the first summand, $(w \mid 1)=0$. In the last sum, $\left(x_{\alpha_{n}} \mid\left[l_{1}\right]_{c} \ldots\left[l_{p}\right]_{c}\left[x_{\beta_{m}}\right]_{c}^{j}\right)$ vanishes, because by earlier results $\left[l_{1}\right]_{c} \ldots\left[l_{p}\right]_{c}\left[x_{\beta_{m}}\right]_{c}^{j}$ is a combination of hyperwords of the PBW basis greater or equal than it and an element of $I(V)$, then we use induction hypothesis and the fact that $I(V)$ is the radical of this bilinear form. Since $x_{\alpha_{n}}, x_{\beta_{m}}^{h_{m}-i}$ are different elements of the PBW basis for $h_{m}-i \neq 1$, we have

$$
(u \mid v)=\left(h_{m}\right)_{q_{\beta m}}\left(w \mid x_{\beta_{1}}^{h_{1}} \ldots x_{\beta_{m-1}}^{h_{m-1}} x_{\beta_{m}}^{h_{m}-1}\right)\left(x_{\alpha_{n}} \mid x_{\beta_{m}}\right) .
$$

This is clearly zero if $\alpha_{n} \neq \beta_{m}$. To see that it is zero also if $\alpha_{n}=\beta_{m}$, note that in that case $w$ and $x_{\beta_{1}}^{h_{1}} \ldots x_{\beta_{m-1}}^{h_{m-1}} x_{\beta_{m}}^{h_{m}-1}$ are different products of PBW generators, and use the induction hypothesis.

Corollary 5.2. If $\alpha \in \Delta^{+}(\mathfrak{B}(V))$, then $x_{\alpha}^{N_{\alpha}}=0$. 
Proof. Let $\left(q_{i j}\right)$ be symmetric. If $u=x_{\alpha_{1}}^{j_{1}} \ldots x_{\alpha_{n}}^{j_{n}}, x_{\alpha_{1}}>\cdots>x_{\alpha_{n}}$, then

$$
(u \mid u)=\prod_{i=1}^{n}\left(j_{i}\right)_{q_{\alpha_{i}}} !\left(x_{\alpha_{i}} \mid x_{\alpha_{i}}\right)^{j_{i}},
$$

where $\left(x_{\alpha} \mid x_{\alpha}\right) \neq 0$ for all $\alpha \in \Delta^{+}(\mathfrak{B}(V))$.

If we consider $u=x_{\alpha}^{N_{\alpha}}$, we have $(u \mid v)=0$ for each $v$ in the PBW basis (because $v$ is an ordered product of $x_{\beta}$ 's different from $u$ ), and $(u \mid u)=0$ since $q_{\alpha} \in \mathbb{G}_{N_{\alpha}}$. Also, $\left(I(V) \mid x_{\alpha}^{N_{\alpha}}\right)=0$, because it is the radical of this bilinear form, so $\left(T(V) \mid x_{\alpha}^{N_{\alpha}}\right)=0$, and then $x_{\alpha}^{N_{\alpha}} \in I(V)$. That is, we have $x_{\alpha}^{N_{\alpha}}=0$ in $\mathfrak{B}(V)$.

For the general case, recall that a diagonal braiding is twist equivalent to a braiding with a symmetric matrix [Andruskiewitsch and Schneider 2002a, Theorem 4.5]. Also, there exists a linear isomorphism between the corresponding Nichols algebras. The corresponding $x_{\alpha}$ are related by a nonzero scalar, because they are an iteration of braided commutators between the hyperwords.

In what follows, $\mathfrak{J}$ denotes the family of $\mathbb{Z}^{\theta}$-graded (hence $\mathbb{N}$-graded) ideals of $T(V)$ that are generated by their components of degree $>1$. For each $I \in \mathfrak{J}$, $\mathfrak{B}=T(V) / I$ is a $\mathbb{Z}^{\theta}$-graded algebra such that $\mathfrak{B}^{0}=\mathrm{k} 1$ and $\mathfrak{B}^{1} \simeq V$.

We shall need some technical results about graded algebras between $T(V)$ and $\mathfrak{B}(V)$. We start with three lemmas dealing with the presence of some important roots in $\Delta(\mathfrak{B})$. Remember that a Lyndon word is a PBW generator in $\mathfrak{B}=T(V) / I$ if it is not a linear combination of greater words modulo $I$ in $T(V)$. We shall relate the absence of some roots in $\Delta(\mathfrak{B})$ (meaning that the Lyndon words of such degrees are linear combinations of greater words modulo $I$ ) with the validity of certain relations in $\mathfrak{B}$.

Lemma 5.3. Let $i, j \in\{1, \ldots, \theta\}$ be distinct, and consider $I \in \mathfrak{J}, \mathfrak{B}=T(V) / I$. Let $D_{k}, k=1, \ldots, \theta$, be skew derivations of $\mathfrak{B}$ as in Proposition 2.1, and assume that $x_{i}^{N}=0$ if $q_{i i}^{n} q_{i j} q_{j i} \neq 1$ for all $n \in \mathbb{N}_{0}$ (where $N=\operatorname{ord} q_{i i}$ ).

There exists $m \in \mathbb{N}$ such that $x_{i}^{m} x_{j}$ is a linear combination of greater hyperwords (for a fixed order such that $x_{i}<x_{j}$ ) modulo I if and only if, in $\mathfrak{B}$.

$$
\left(\operatorname{ad}_{c} x_{i}\right)^{m_{i j}+1} x_{j}=0 \text {. }
$$

Proof. If $\left(\operatorname{ad}_{c} x_{i}\right)^{m} x_{j}=0$, there exist $a_{k} \in \mathrm{k}$ such that

$$
0=\left[x_{i}^{m} x_{j}\right]_{c}=\left(\operatorname{ad}_{c} x_{i}\right)^{m} x_{j}=x_{i}^{m} x_{j}+\sum_{k=0}^{m-1} a_{k} x_{i}^{k} x_{j} x_{i}^{m-k} .
$$

Conversely, suppose there exists $m \in \mathbb{N}$ such that $x_{i}^{m} x_{j}$ is a linear combination of greater hyperwords modulo $I$. Let

$$
n=\min \left\{m \in \mathbb{N}: x_{i}^{m} x_{j} \text { is a linear combination of greater hyperwords }\right\} .
$$


If $x_{i}^{n}=0$, then $q_{i i}$ is a root of unity. In this case, if $N$ is the order of $q_{i i}$, then $x_{i}^{N}=0$ and $x_{i}^{N-1} \neq 0$. Also,

$$
\left(\operatorname{ad}_{c} x_{i}\right)^{N} x_{j}=x_{i}^{N} x_{j}+\sum_{s=1}^{N-1}\left(\begin{array}{c}
N \\
s
\end{array}\right)_{q_{i i}}+x_{j} x_{i}^{N}=0,
$$

because $\left(\begin{array}{l}N \\ s\end{array}\right)_{q_{i i}}=0$ for $0<s<N$. Hence, we can assume $x_{i}^{n} \neq 0$ and $(n)_{q_{i i}} ! \neq 0$.

Note that $\left[x_{i}^{n-k} x_{j} x_{i}^{k}\right]_{c}=\left[x_{i}^{n-k} x_{j}\right]_{c} x_{i}^{k}$. Since $\mathfrak{B}$ is graded, $x_{i}^{n} x_{j}$ is a linear combination of terms $x_{i}^{n-k} x_{j} x_{i}^{k}, 0 \leq k<n$. Hence there exist $\alpha_{k} \in \mathrm{k}$ such that

$$
\left[x_{i}^{n} x_{j}\right]_{c}=\sum_{k=1}^{n} \alpha_{k}\left[x_{i}^{n-k} x_{j}\right]_{c} x_{i}^{k} .
$$

Applying $D_{i}$ we obtain

$$
0=D_{i}\left(\left[x_{i}^{n} x_{j}\right]_{c}\right)=\sum_{k=1}^{n} \alpha_{k} D_{i}\left(\left[x_{i}^{n-k} x_{j}\right]_{c} x_{i}^{k}\right)=\sum_{k=1}^{n} \alpha_{k}(k)_{q_{i i}}\left[x_{i}^{n-k} x_{j}\right]_{c} x_{i}^{k} .
$$

By the hypothesis about $n, \alpha_{1}=0$. Since $(n)_{q_{i i}} ! \neq 0$, applying $D_{i}$ several times we conclude that $\alpha_{k}=0$ for $k=2, \ldots, n$. Hence $\left[x_{i}^{n} x_{j}\right]_{c}=0$.

Recall that (5-3) holds in $\mathfrak{B}(V)$, for $1 \leq i \neq j \leq \theta$.

The second lemma is related to Dynkin diagrams of a standard braiding which have two consecutive simple edges.

Lemma 5.4. Let $I \in \mathfrak{J}$ and $\mathfrak{B}=T(V) / I$. Assume that

- there exist skew derivations $D_{k}$ in $\mathfrak{B}$ as in Proposition 2.1;

- there exist different $j, k, l \in\{1, \ldots, \theta\}$ such that $m_{k j}=m_{k l}=1, m_{j l}=0$;

- $\left(\operatorname{ad} x_{k}\right)^{2} x_{j}=\left(\operatorname{ad} x_{k}\right)^{2} x_{l}=\left(\operatorname{ad} x_{j}\right) x_{l}=0$ hold in $\mathfrak{B}$;

- $x_{k}^{2}=0$ if $q_{k k} q_{k j} q_{j k} \neq 1$ or $q_{k k} q_{k l} q_{l k} \neq 1$.

(1) If we order the letters $x_{1}, \ldots, x_{\theta}$ such that $x_{j}<x_{k}<x_{l}$, then $x_{j} x_{k} x_{l} x_{k}$ is a linear combination of greater words modulo I if and only if, in $\mathfrak{B}$,

$$
\left[\left(\operatorname{ad} x_{j}\right)\left(\operatorname{ad} x_{k}\right) x_{l}, x_{k}\right]_{c}=0 .
$$

(2) If $V$ is standard and $q_{k k} \neq-1$, then (5-4) holds in $\mathfrak{B}$.

(3) If $V$ is standard and $\operatorname{dim} \mathfrak{B}(V)<\infty$, then (5-4) holds in $\mathfrak{B}=\mathfrak{B}(V)$.

Proof. $(1)(\Leftarrow)$ If $(5-4)$ holds, then $x_{j} x_{k} x_{l} x_{k}$ is a linear combination of greater words, by Remark 1.7, and

$$
\left[x_{j} x_{k} x_{l} x_{k}\right]_{c}=\left[\left[x_{j} x_{k} x_{l}\right]_{c}, x_{k}\right]_{c}=\left[\left(\operatorname{ad} x_{j}\right)\left(\operatorname{ad} x_{k}\right) x_{l}, x_{k}\right]_{c} .
$$


$(\Rightarrow)$ If $x_{j} x_{k} x_{l} x_{k}$ is a linear combination of greater words, then the hyperword $\left[x_{j} x_{k} x_{l} x_{k}\right]_{c}$ is a linear combination of hyperwords corresponding to words greater than $x_{j} x_{k} x_{l} x_{k}$ (of the same degree, because $\mathfrak{B}$ is homogeneous); this follows from Remark 1.7. Since $\left(\operatorname{ad} x_{k}\right)^{2} x_{j}=\left(\operatorname{ad} x_{k}\right)^{2} x_{l}=\left(\operatorname{ad} x_{j}\right) x_{l}=0$, we do not consider hyperwords with $x_{j} x_{k}^{2}, x_{k}^{2} x_{l}$ and $x_{j} x_{l}$ as factors of the corresponding words. Then $\left[x_{j} x_{k} x_{l} x_{k}\right]_{c}$ is a linear combination of

$$
\begin{array}{rlrl}
{\left[x_{k} x_{l} x_{k} x_{j}\right]_{c}} & =\left[x_{k} x_{l}\right]_{c} x_{k} x_{j}, & {\left[x_{l} x_{k} x_{j} x_{k}\right]_{c}} & =x_{l} x_{k}\left[x_{j} x_{k}\right]_{c}, \\
{\left[x_{k} x_{j} x_{k} x_{l}\right]_{c}} & =x_{k}\left[x_{j} x_{k} x_{l}\right]_{c}, & {\left[x_{l} x_{k}^{2} x_{j}\right]_{c}=x_{l} x_{k}^{2} x_{j} .}
\end{array}
$$

Since $D_{j}\left(\left[x_{j} x_{k} x_{l} x_{k}\right]_{c}\right)=D_{j}\left(x_{k}\left[x_{j} x_{k} x_{l}\right]_{c}\right)=D_{j}\left(x_{l} x_{k}\left[x_{j} x_{k}\right]_{c}\right)=0$, in that linear combination there are no hyperwords ending in $x_{j}$; indeed,

$$
D_{j}\left(\left[x_{k} x_{l}\right]_{c} x_{k} x_{j}\right)=\left[x_{k} x_{l}\right]_{c} x_{k}, \quad D_{j}\left(x_{l} x_{k}^{2} x_{j}\right)=x_{l} x_{k}^{2},
$$

and $\left[x_{k} x_{l}\right]_{c} x_{k}, x_{l} x_{k}^{2}$ are linearly independent. Therefore, there exist $\alpha, \beta \in \mathrm{k}$ such that

$$
\left[x_{j} x_{k} x_{l} x_{k}\right]_{c}=\alpha x_{l} x_{k}\left[x_{j} x_{k}\right]_{c}+\beta x_{k}\left[x_{j} x_{k} x_{l}\right]_{c} .
$$

Applying $D_{l}$, we have

$$
0=\alpha q_{k j} q_{k k} x_{l}\left[x_{j} x_{k}\right]_{c}+\alpha\left(1-q_{k j} q_{j k}\right) x_{l} x_{k} x_{j}+\beta q_{k k} q_{k j} q_{k l}\left[x_{j} x_{k} x_{l}\right]_{c} .
$$

Now $x_{l}\left[x_{j} x_{k}\right]_{c}, x_{l} x_{k} x_{j}$ and $\left[x_{j} x_{k} x_{l}\right]_{c}$ are linearly independent by Lemma 2.7, so $\alpha=\beta=0$.

(2) We assume that some quantum Serre relations hold in $\mathfrak{B}$; using them we get

$$
\begin{aligned}
x_{j} x_{k} x_{l} x_{k}= & q_{k l}^{-1}\left(1+q_{k k}\right)^{-1} x_{j} x_{k}^{2} x_{l}+q_{k k} q_{k j}\left(1+q_{k k}\right)^{-1} x_{j} x_{l} x_{k}^{2} \\
= & q_{k k}^{-1} q_{k j}^{-1} q_{k l}^{-1} x_{k} x_{j} x_{k} x_{l}+q_{k k}^{-1} q_{k j}^{-1} q_{k l}^{-1}\left(1+q_{k k}\right)^{-1} x_{k}^{2} x_{j} x_{l} \\
& \quad+q_{k k} q_{k l} q_{j k}\left(1+q_{k k}\right)^{-1} x_{l} x_{j} x_{k}^{2} .
\end{aligned}
$$

It follows that $x_{k} x_{j} x_{k} x_{l} \notin G_{I}$ for an order such that $x_{j}<x_{k}<x_{l}$. Also, $x_{j} x_{l} x_{k}^{2} \notin G_{I}$, since $\left(\operatorname{ad}_{c} x_{j}\right) x_{l}=0$ and (5-4) is valid by part (1).

(3) If $V$ is a standard braided vector space satisfying the conditions of the lemma, consider $V_{k}$ as the braided vector space obtained transforming by $s_{k}$. Then $\widetilde{m}_{j l}=0$. Therefore $\mathbf{e}_{j}+\mathbf{e}_{l} \notin \Delta^{+}\left(\mathfrak{B}\left(V_{k}\right)\right)$, so $s_{k}\left(\mathbf{e}_{j}+\mathbf{e}_{l}\right)=2 \mathbf{e}_{k}+\mathbf{e}_{j}+\mathbf{e}_{l} \notin \Delta^{+}(\mathfrak{B}(V))$. It follows that $x_{j} x_{k} x_{l} x_{k}$ is a linear combination of greater words, since it is a Lyndon word when we consider an order such that $x_{j}<x_{k}<x_{l}$.

We now prove two relations involving the double edge in the Dynkin diagram of a standard braiding of type $B_{\theta}$.

Lemma 5.5. Let $I \in \mathfrak{J}$ and $\mathfrak{B}=T(V) / I$. Assume that

- there exist $j \neq k \in\{1, \ldots, \theta\}$ such that $m_{k j}=2, m_{j k}=1$; 
- there exist skew derivations as in Proposition 2.1;

- the following relations hold in $\mathfrak{B}$ :

$$
\begin{aligned}
& \left(\operatorname{ad} x_{k}\right)^{3} x_{j}=\left(\operatorname{ad} x_{j}\right)^{2} x_{k}=0 ; \\
& x_{k}^{3}=x_{j}^{2}=0 \quad \text { if } q_{k k}^{3}=q_{j j}^{2}=1 .
\end{aligned}
$$

(1) If we order the letters $x_{1}, \ldots, x_{\theta}$ such that $x_{k}<x_{j}$, then $x_{k}^{2} x_{j} x_{k} x_{j}$ is a linear combination of greater words modulo I if and only if, in $\mathfrak{B}$,

$$
\left[\left(\operatorname{ad} x_{k}\right)^{2} x_{j},\left(\operatorname{ad} x_{k}\right) x_{j}\right]_{c}=0 .
$$

(2) If $V$ is standard, $q_{j j} \neq-1$ and $q_{k k}^{2} q_{k j} q_{j k}=1$, then (5-6) holds in $\mathfrak{B}$.

(3) If $V$ is standard and $\operatorname{dim} \mathfrak{B}(V)<\infty$, then (5-6) holds in $\mathfrak{B}=\mathfrak{B}(V)$.

Proof. $(1)(\Leftarrow)$ If $(5-6)$ holds in $\mathfrak{B}$, then $x_{k}^{2} x_{j} x_{k} x_{j}$ is a linear combination of greater words. This follows from Remark 1.7, and

$$
\left[x_{k}^{2} x_{j} x_{k} x_{j}\right]_{c}=\left[\left[x_{k}^{2} x_{j}\right]_{c},\left[x_{k} x_{j}\right]_{c}\right]_{c}=\left[\left(\operatorname{ad} x_{k}\right)^{2} x_{j},\left(\operatorname{ad} x_{k}\right) x_{j}\right]_{c} .
$$

$(\Rightarrow)$ If $x_{k}^{2} x_{j} x_{k} x_{j}$ is a linear combination of greater words, then $\left[x_{k}^{2} x_{j} x_{k} x_{j}\right]_{c}$ is a linear combination of hyperwords corresponding to words greater than $x_{k}^{2} x_{j} x_{k} x_{j}$ (of the same degree, because $\mathfrak{B}$ is homogeneous).

First, there are no hyperwords whose corresponding words have factors $x_{k}^{3} x_{j}$ or $x_{k} x_{j}^{2}$, by (5-5). Since $\left[x_{k}^{2} x_{j} x_{k} x_{j}\right]_{c} \in \operatorname{ker} D_{k}$ and

$$
\begin{aligned}
D_{k}\left(x_{j}\left[x_{k}^{2} x_{j}\right]_{c} x_{k}\right) & =x_{j}\left[x_{k}^{2} x_{j}\right]_{c}, \\
D_{k}\left(\left[x_{k} x_{j}\right]_{c}^{2} x_{k}\right) & =\left[x_{k} x_{j}\right]_{c}^{2}, \\
D_{k}\left(x_{j}\left[x_{k} x_{j}\right]_{c} x_{k}^{2}\right) & =\left(1+q_{k k}\right) x_{j}\left[x_{k} x_{j}\right]_{c} x_{k},
\end{aligned}
$$

in that linear combination there are no hyperwords ending in $x_{k}$, except $x_{j}^{2} x_{k}^{3}$ if $q_{k k} \in \mathbb{G}_{3}$. We consider $q_{j j} \neq-1$ if $q_{k k} \in \mathbb{G}_{3}$, since otherwise $x_{j}^{2} x_{k}^{3}=0$ by assumption. Then there exist $\alpha, \alpha^{\prime} \in \mathrm{k}$ such that

$$
\left[x_{k}^{2} x_{j} x_{k} x_{j}\right]_{c}=\alpha\left[x_{k} x_{j} x_{k}^{2} x_{j}\right]_{c}+\alpha^{\prime} x_{j}^{2} x_{k}^{3}=\alpha\left[x_{k} x_{j}\right]_{c}\left[x_{k}^{2} x_{j}\right]_{c}+\alpha^{\prime} x_{j}^{2} x_{k}^{3} .
$$

We prove by direct calculation that $D_{j}\left(\left[x_{k}^{2} x_{j} x_{k} x_{j}\right]_{c}\right)=0$. Applying $D_{j}$ to the previous equality,

$$
\begin{aligned}
0= & \alpha^{\prime}\left(1+q_{j j}\right) x_{j} x_{k}^{3}+\chi\left(\mathbf{e}_{k}+\mathbf{e}_{j}, 2 \mathbf{e}_{k}+\mathbf{e}_{j}\right) \alpha\left(\operatorname{ad} x_{k}\right)^{2}\left(x_{j}\right) x_{k} \\
& +\left(1-q_{k j} q_{j k}\right)\left(1-q_{k k} q_{k j} q_{j k}\right) \alpha\left(\operatorname{ad} x_{k}\right)\left(x_{j}\right) x_{k}^{2},
\end{aligned}
$$

where we use that $\left(\operatorname{ad} x_{k}\right)^{3}\left(x_{j}\right)=0$ and

$$
x_{k}\left(\operatorname{ad} x_{k}\right)^{m}\left(x_{j}\right)=\left(\operatorname{ad} x_{k}\right)^{m+1}\left(x_{j}\right)+q_{k k}^{m} q_{k j}\left(\operatorname{ad} x_{k}\right)^{m}\left(x_{j}\right) x_{k} .
$$


Since $\left(1-q_{k j} q_{j k}\right)\left(1-q_{k k} q_{k j} q_{j k}\right) \neq 0$ and $\left(\operatorname{ad} x_{k}\right)^{2}\left(x_{j}\right) x_{k},\left(\operatorname{ad} x_{k}\right)\left(x_{j}\right) x_{k}^{2}, x_{j} x_{k}^{3}$ are linearly independent, it follows that $\alpha=\alpha^{\prime}=0$.

(2) Using $\left(\operatorname{ad} x_{j}\right)^{2} x_{k}=0$ in the first equality and $\left(\operatorname{ad} x_{k}\right)^{3} x_{j}=0$ in the last expression,

$$
\begin{aligned}
x_{k}^{2} x_{j} x_{k} x_{j} & =\left(1+q_{j j}\right)^{-1} q_{j k}^{-1} x_{k}^{2} x_{j}^{2} x_{k}+\left(1+q_{j j}\right)^{-1} q_{j k} q_{j j} x_{k}^{3} x_{j}^{2} \\
& \in(3)_{q_{k k}}\left(1+q_{j j}\right)^{-1} q_{k j} q_{j k} q_{j j} x_{k}^{2} x_{j} x_{k} x_{j}+\mathrm{k} \mathbb{X}_{>x_{k}^{2} x_{j} x_{k} x_{j}} .
\end{aligned}
$$

Suppose that $(3)_{q_{k k}}\left(1+q_{j j}\right)^{-1} q_{k j} q_{j k} q_{j j}=1$; that is, $(3)_{q_{k k}}=\left(1+q_{j j}\right)$. Then $q_{j j}=q_{k k}+q_{k k}^{2}$, so

$$
1=q_{j j} q_{k j} q_{j k}=q_{k k} q_{k j} q_{j k}+q_{k k}^{2} q_{k j} q_{j k}=q_{k k} q_{k j} q_{j k}+1,
$$

which is a contradiction since $q_{k k} q_{k j} q_{j k} \in \mathrm{k}^{\times}$. It follows that $x_{k}^{2} x_{j} x_{k} x_{j}$ is a linear combination of greater words, so (5-6) follows by previous item.

(3) If $V$ is a standard braided vector space, and we consider $V_{j}$ as the braided vector space obtained transforming by $s_{j}$, then $\widetilde{m}_{k j}=2$. Therefore, $3 \mathbf{e}_{k}+\mathbf{e}_{j} \notin \Delta^{+}\left(\mathfrak{B}\left(V_{k}\right)\right)$, so $s_{j}\left(3 \mathbf{e}_{k}+\mathbf{e}_{j}\right)=3 \mathbf{e}_{k}+2 \mathbf{e}_{j} \notin \Delta^{+}(\mathfrak{B}(V))$. Since $x_{k}^{2} x_{j} x_{k} x_{j}$ is a Lyndon word of degree $3 \mathbf{e}_{k}+2 \mathbf{e}_{j}$ if $x_{k}<x_{j}$, then it is a linear combination of greater words.

Lemma 5.6. Let $I \in \mathfrak{J}$ and $\mathfrak{B}=T(V) / I$. Assume that

- there exist different $j, k, l \in\{1, \ldots, \theta\}$ such that $m_{k j}=2, m_{j k}=m_{j l}=m_{l j}=1$, $m_{k l}=0$;

- there exist skew derivations in $\mathfrak{B}$ as in Proposition 2.1;

- the following relations hold in $\mathfrak{B}:(5-4),(5-6)$,

$$
\begin{aligned}
& \left(\operatorname{ad} x_{k}\right)^{3} x_{j}=\left(\operatorname{ad} x_{j}\right)^{2} x_{k}=\left(\operatorname{ad} x_{j}\right)^{2} x_{l}=\left(\operatorname{ad} x_{k}\right) x_{l}=0, \\
& x_{k}^{3}=x_{j}^{2}=0 \text { if } q_{k k}^{3}=q_{j j}^{2}=1 .
\end{aligned}
$$

(1) If we order the letters $x_{1}, \ldots, x_{\theta}$ so that $x_{k}<x_{j}<x_{l}$, then $x_{k}^{2} x_{j} x_{l} x_{k} x_{j}$ is a linear combination of greater words modulo I if and only if, in $\mathfrak{B}$,

$$
\left[\left(\operatorname{ad} x_{k}\right)^{2}\left(\operatorname{ad} x_{j}\right) x_{l},\left(\operatorname{ad} x_{k}\right) x_{j}\right]_{c}=0 .
$$

(2) If $V$ is a standard braided vector space and $q_{k k} \notin \mathbb{G}_{3}, q_{j j} \neq-1$, then (5-8) holds in $\mathfrak{B}$.

(3) If $V$ is standard and $\operatorname{dim} \mathfrak{B}(V)<\infty$, then (5-8) holds in $\mathfrak{B}(V)$.

Proof. (1) $(\Leftarrow)$ As in the last two lemmas, if (5-8) is valid, then $x_{k}^{2} x_{j} x_{l} x_{k} x_{j}$ is a linear combination of greater words, by Remark 1.7, and

$$
\left[x_{k}^{2} x_{j} x_{l} x_{k} x_{j}\right]_{c}=\left[\left(\operatorname{ad} x_{k}\right)^{2}\left(\operatorname{ad} x_{j}\right) x_{l},\left(\operatorname{ad} x_{k}\right) x_{j}\right]_{c} .
$$


$(\Rightarrow)$ Suppose that $x_{k}^{2} x_{j} x_{l} x_{k} x_{j}$ is a linear combination of greater words. Then $\left[x_{k}^{2} x_{j} x_{l} x_{k} x_{j}\right]_{c}$ is a linear combination of hyperwords corresponding to words greater than $x_{k}^{2} x_{j} x_{l} x_{k} x_{j}$ (of the same degree, because $\mathfrak{B}$ is homogeneous). We discard those words which have $x_{k} x_{l}, x_{k}^{3} x_{j}, x_{k} x_{j}^{2}, x_{j}^{2} x_{l}, x_{k} x_{j} x_{l} x_{j}$ and $x_{k}^{2} x_{j} x_{k} x_{j}$, in view of our hypotheses about $\mathfrak{B}$.

Since $D_{k}\left(\left[x_{k}^{2} x_{j} x_{l} x_{k} x_{j}\right]_{c}\right)=0$, the coefficients of hyperwords corresponding to words ending in $x_{k}$ are 0 , as in Lemma 5.5, except for $\left[x_{j} x_{l}\right]_{c} x_{j} x_{k}^{3}, x_{l} x_{j}^{2} x_{k}^{3}$, if $q_{k k} \in \mathbb{G}_{3}$. Thus

$$
\begin{aligned}
{\left[x_{k}^{2} x_{j} x_{l} x_{k} x_{j}\right]_{c}=\alpha\left[x_{k} x_{j}\right]_{c}\left[x_{k}^{2} x_{j} x_{l}\right]_{c} } & +\beta\left[x_{k} x_{j} x_{l}\right]_{c}\left[x_{k}^{2} x_{j}\right]_{c} \\
& +\gamma x_{l}\left[x_{k} x_{j}\right]_{c}\left[x_{k}^{2} x_{j}\right]_{c}+\mu\left[x_{j} x_{l}\right]_{c} x_{j} x_{k}^{3}+v x_{l} x_{j}^{2} x_{k}^{3} .
\end{aligned}
$$

By direct calculation, $D_{j}\left(\left[x_{k}^{2} x_{j} x_{l} x_{k} x_{j}\right]_{c}\right)=D_{j}\left(\left[x_{k}^{2} x_{j} x_{l}\right]_{c}\right)=D_{j}\left(\left[x_{k} x_{j} x_{l}\right]_{c}\right)=0$, so applying $D_{j}$ to the previous equality we get

$$
\begin{aligned}
& 0=\alpha q_{j k}^{2} q_{j j} q_{j l} x_{j}\left[x_{k}^{2} x_{j} x_{l}\right]_{c}+\beta\left(1-q_{k k} q_{k j} q_{j k}\right)\left(1-q_{k k}^{2} q_{k j} q_{j k}\right)\left[x_{k} x_{j} x_{l}\right]_{c} x_{k}^{2} \\
&+\gamma\left(1-q_{k k} q_{k j} q_{j k}\right)\left(1-q_{k k}^{2} q_{k j} q_{j k}\right) x_{l}\left[x_{k} x_{j}\right]_{c} x_{k}^{2}+\gamma q_{j k}^{2} q_{j j} x_{l} x_{j}\left[x_{k}^{2} x_{j}\right]_{c} \\
&+\mu\left[x_{j} x_{l}\right]_{c} x_{k}^{3}+v\left(1+q_{j j}\right) x_{l} x_{j} x_{k}^{3},
\end{aligned}
$$

Note that $v=0$ if $q_{j j} \neq-1$; otherwise, $x_{j}^{2}=0$ by hypothesis, so we can discard this last summand. The other hyperwords appearing in this expression are linearly independent, since the corresponding words are linearly independent by Lemma 2.7. Thus $\alpha=\beta=\gamma=\mu=0$.

(2) If $q_{k k} \notin \mathbb{G}_{3}$ and $q_{j j} \neq-1$, then $x_{k}^{2} x_{j} x_{l} x_{k} x_{j}$ is a linear combination of greater words, as can be seen using the quantum Serre relations in a way similar to that in Lemma 5.6. Now apply part (1).

(3) If $V$ is a standard braided vector space, consider $V_{k}$ as the braided vector space obtained transforming by $s_{k}$. Then $\widetilde{m}_{k j}=2$. Therefore, $\mathbf{e}_{k}+2 \mathbf{e}_{j}+\mathbf{e}_{l} \notin \Delta^{+}\left(\mathfrak{B}\left(V_{k}\right)\right)$ by Lemma 5.5, so $s_{k}\left(\mathbf{e}_{k}+2 \mathbf{e}_{j}+\mathbf{e}_{l}\right)=3 \mathbf{e}_{k}+2 \mathbf{e}_{j}+\mathbf{e}_{l} \notin \Delta^{+}(\mathfrak{B}(V))$. Since $x_{k}^{2} x_{j} x_{l} x_{k} x_{j}$ is a Lyndon word, it follows that it is a linear combination of greater words, and we apply (1).

We now give explicit formulas for the comultiplication of these hyperwords.

Lemma 5.7. Consider the structure of graded braided Hopf algebra of $T(V)$ (see Section 2A). For all $k \neq j$,

$$
\begin{aligned}
\Delta\left(\left(\operatorname{ad} x_{k}\right)^{m_{k j}+1} x_{j}\right)=\left(\operatorname{ad} x_{k}\right)^{m_{k j}+1} x_{j} \otimes 1 & +1 \otimes\left(\operatorname{ad} x_{k}\right)^{m_{k j}+1} x_{j} \\
& +\prod_{1 \leq t \leq m_{k j}}\left(1-q_{k k}^{t} q_{k j} q_{j k}\right) x_{k}^{m_{i j}+1} \otimes x_{j} .
\end{aligned}
$$

Proof. We have $F_{k}\left(\left(\operatorname{ad} x_{k}\right)^{m_{k j}+1} x_{j}\right)=0$ by the definition of $m_{k j}$ and (2-5). Also, $F_{l}\left(\left(\operatorname{ad} x_{k}\right)^{m_{k j}+1} x_{j}\right)$ for $l \neq k$ by $(2-6)$ and the properties of $F_{l}$, so 


$$
\Delta_{1, m_{k j}}\left(\left(\operatorname{ad} x_{k}\right)^{m_{k j}+1} x_{j}\right)=\sum_{l=1}^{\theta} x_{l} \otimes F_{l}\left(\left(\operatorname{ad} x_{k}\right)^{m_{k j}+1} x_{j}\right)=0
$$

Now $D_{k}\left(\left[x_{k}^{i} x_{j}\right]_{c} x_{k}^{s-i}\right)=0$ from (2-3), and from (2-4)

$$
D_{j}\left(\left[x_{k}^{i} x_{j}\right]_{c} x_{k}^{s-i}\right)=\prod_{1 \leq t \leq m_{k j}}\left(1-q_{k k}^{t} q_{k j} q_{j k}\right) x_{k}^{m_{i j}+1}
$$

so we deduce that

$$
\Delta_{m_{k j}, 1}\left(\left(\operatorname{ad} x_{k}\right)^{m_{k j}+1} x_{j}\right)=\prod_{1 \leq t \leq m_{k j}}\left(1-q_{k k}^{t} q_{k j} q_{j k}\right) x_{k}^{m_{i j}+1} \otimes x_{j} .
$$

Since hyperwords form a basis of $T(V)$, we can write, for each $1<s<m_{k j}$,

$$
\begin{aligned}
\Delta_{m_{k j}+1-s, s} & \left(\left(\operatorname{ad} x_{k}\right)^{m_{k j}+1} x_{j}\right) \\
= & \sum_{t=0}^{m_{k j}+1-s} \varepsilon_{s t}\left[x_{k}^{t} x_{j}\right]_{c} x_{k}^{m_{k j}+1-s-t} \otimes x_{k}^{s}+\sum_{p=0}^{s} \rho_{s p} x_{k}^{m_{k j}+1-s} \otimes\left[x_{k}^{s-p} x_{j}\right]_{c} x_{k}^{p},
\end{aligned}
$$

for some $\varepsilon_{s t}, \rho_{s p} \in \mathrm{k}$. Then, for each $0 \leq t \leq m_{k j}+1-s$,

$$
\begin{aligned}
0 & =\left(\left(\operatorname{ad} x_{k}\right)^{m_{k j}+1} x_{j} \mid\left[x_{k}^{t} x_{j}\right]_{c} x_{k}^{m_{k j}+1-t} x_{k}^{s}\right) \\
& =\left(\left(\left(\operatorname{ad} x_{k}\right)^{m_{k j}+1} x_{j}\right)_{(1)} \mid\left[x_{k}^{t} x_{j}\right]_{c} x_{k}^{m_{k j}+1-t-s}\right)\left(\left(\left(\operatorname{ad} x_{k}\right)^{m_{k j}+1} x_{j}\right)_{(2)} \mid x_{k}^{s}\right) \\
& =\varepsilon_{s t}\left(\left[x_{k}^{t} x_{j}\right]_{c} x_{k}^{m_{k j}+1-t-s} \mid\left[x_{k}^{t} x_{j}\right]_{c} x_{k}^{m_{k j}+1-t-s}\right)\left(x_{k}^{s} \mid x_{k}^{s}\right) \\
& =\varepsilon_{s t}\left(m_{k j}+1-s-t\right)_{q_{k k}} !(s)_{q_{k k}} !\left(\left[x_{k}^{t} x_{j}\right]_{c} \mid\left[x_{k}^{t} x_{j}\right]_{c}\right),
\end{aligned}
$$

where we have used that $\left(\operatorname{ad} x_{k}\right)^{m_{k j}+1} x_{j} \in I(V)$ for the first equality, (1-8) for the second, (1-10) and the orthogonality between increasing products of hyperwords for the third, and (5-2) for the last. Since

$$
\left(m_{k j}+1-s-t\right)_{q_{k}} !(s)_{q_{k}} !\left(\left[x_{k}^{t} x_{j}\right]_{c} \mid\left[x_{k}^{t} x_{j}\right]_{c}\right) \neq 0,
$$

we conclude that $\varepsilon_{s t}=0$ for all $0 \leq t \leq m_{k j}+1-s$. In a similar way, $\rho_{s p}=0$ for all $0 \leq p \leq s$, so we obtain (5-9).

Lemma 5.8. Let $\mathfrak{B}$ be a braided graded Hopf algebra provided with an inclusion of braided vector spaces $V \hookrightarrow \mathscr{P}(\mathfrak{B})$. Assume that

- there exist $1 \leq j \neq k \neq l \leq \theta$ such that $m_{k j}=m_{k l}=1, m_{j l}=0$;

- $\left(\operatorname{ad} x_{k}\right)^{2} x_{j}=\left(\operatorname{ad} x_{k}\right)^{2} x_{l}=\left(\operatorname{ad} x_{j}\right) x_{l}=0$ in $\mathfrak{B}$;

- $x_{k}^{2}=0$ if $q_{k k} q_{k j} q_{j k} \neq 1$ or $q_{k k} q_{k l} q_{l k} \neq 1$.

Then $u:=\left[\left(\operatorname{ad} x_{j}\right)\left(\operatorname{ad} x_{k}\right) x_{l}, x_{k}\right]_{c} \in \mathscr{P}(\mathfrak{B})$. 
Proof. From (2-3), $D_{j}(u)=0$. Also, $D_{k}\left(\left(\operatorname{ad} x_{j}\right)\left(\operatorname{ad} x_{k}\right) x_{l}\right)=0$, so

$$
D_{k}(u)=\left(1-q_{k k}^{2} q_{j k} q_{k j} q_{k l} q_{l k}\right)\left(\operatorname{ad} x_{j}\right)\left(\operatorname{ad} x_{k}\right) x_{l}=0 .
$$

From (2-4) and the properties of $D_{l}$ we have

$$
\begin{aligned}
D_{l}(u) & =q_{l k}\left(1-q_{k l} q_{l k}\right)\left[x_{j} x_{k}\right]_{c} x_{k}-q_{j k} q_{k k} q_{l k}\left(1-q_{k l} q_{l k}\right) x_{k}\left[x_{j} x_{k}\right]_{c} \\
& =q_{l k}\left(1-q_{l k} q_{k l}\right)\left[\left[x_{j} x_{k}\right]_{c}, x_{k}\right]_{c}=0 .
\end{aligned}
$$

Then $\Delta_{31}(u)=0$. From (2-6) and the properties of $F_{k}$ and $F_{l}$, we have $F_{k}(u)=$ $F_{l}(u)=0$. Using $(2-5)$, we have

$$
\begin{aligned}
F_{j}(u) & =\left(1-q_{j k} q_{k j}\right)\left[x_{k} x_{l}\right]_{c} x_{k}-q_{j k} q_{k k} q_{l k} q_{k j}\left(1-q_{j k} q_{k j}\right) x_{k}\left[x_{k} x_{l}\right]_{c} \\
& =\left(1-q_{l k} q_{k l}\right)\left(1-q_{k j} q_{j k} q_{k k}^{2} q_{l k} q_{j k}\right)\left[x_{k} x_{l}\right]_{c} x_{k}=0 .
\end{aligned}
$$

Thus $\Delta_{13}(u)=0$ as well.

Also, we have

$$
\Delta(u)=\Delta\left(\left(\operatorname{ad} x_{j}\right)\left(\operatorname{ad} x_{k}\right) x_{l}\right) \Delta\left(x_{k}\right)-q_{\mathbf{e}_{k}+\mathbf{e}_{j}+\mathbf{e}_{j}, \mathbf{e}_{j}} \Delta\left(x_{k}\right) \Delta\left(\left(\operatorname{ad} x_{j}\right)\left(\operatorname{ad} x_{k}\right) x_{l}\right),
$$

and looking at the terms in $\mathfrak{B}^{2} \otimes \mathfrak{B}^{2}$,

$$
\begin{aligned}
& \Delta_{2,2}(u)=\left(1-q_{l k} q_{k l}\right)\left[x_{j} x_{k}\right]_{c} \otimes\left(x_{l} x_{k}-q_{k j} q_{j k} q_{k k}^{2} q_{l k} x_{k} x_{l}\right) \\
&+\left(1-q_{k j} q_{j k}\right) q_{l k} q_{k k}\left(x_{j} x_{k}-q_{j k} x_{k} x_{j}\right) \otimes\left[x_{k} x_{l}\right]_{c} \\
&=\left(1-q_{k j} q_{j k}-\left(1-q_{l k} q_{k l}\right) q_{k k} q_{j k} q_{k j}\right) q_{l k} q_{k k}\left[x_{j} x_{k}\right]_{c} \otimes\left[x_{k} x_{l}\right]_{c} .
\end{aligned}
$$

Now a calculation shows that $u \in \mathscr{P}(\mathfrak{B})$ :

$$
\begin{aligned}
1-q_{k j} q_{j k}-\left(1-q_{l k} q_{k l}\right) q_{k k} q_{j k} q_{k j} & =1-q_{k j} q_{j k}-q_{k k} q_{j k} q_{k j}+q_{k k}^{-1} \\
& =q_{k k}^{-1}\left(1+q_{k k}\right)\left(1-q_{k k} q_{k j} q_{j k}\right)=0 .
\end{aligned}
$$

Lemma 5.9. Let $\mathfrak{B}$ be a braided graded Hopf algebra provided with an inclusion of braided vector spaces $V \hookrightarrow \mathscr{P}(\mathfrak{B})$. Assume that

- there exist $1 \leq k \neq j \leq \theta$ such that $m_{k j}=2, m_{j k}=1$;

- $\left(\operatorname{ad} x_{s}\right)^{m_{s t}+1} x_{t}=0$, for all $1 \leq s \neq t \leq \theta$ in $\mathfrak{B}$;

- $x_{s}^{m_{s t}+1}=0$ for each $s$ such that $q_{s s}^{m_{s t}} q_{s t} q_{t s} \neq 1$, for some $t \neq s$.

(a) If $v:=\left[\left(\operatorname{ad} x_{k}\right)^{2} x_{j},\left(\operatorname{ad} x_{k}\right) x_{j}\right]_{c}$, there exists $b \in \mathrm{k}$ such that

$$
\Delta(v)=v \otimes 1+1 \otimes v+b\left(1-q_{k k}^{2} q_{k j}^{2} q_{j k}^{2} q_{j j}\right) x_{k}^{3} \otimes x_{j}^{2} .
$$

(b) Assume there exist $l \neq j, k$ such that $m_{j l}=m_{l j}=1, m_{k l}=m_{l k}=0$, and that (5-4) is valid in $\mathfrak{B}$. Set

$$
w:=\left[\left(\operatorname{ad} x_{k}\right)^{2}\left(\operatorname{ad} x_{j}\right) x_{l},\left(\operatorname{ad} x_{k}\right) x_{j}\right]_{c} .
$$


Then there exist constants $b_{1}, b_{2} \in \mathrm{k}$ such that

$$
\Delta(w)=w \otimes 1+1 \otimes w+b_{1} v \otimes x_{l}+b_{2}\left(1-q_{k k}^{2} q_{k j} q_{j k}\right) x_{k}^{3} \otimes\left(\left(\operatorname{ad} x_{j}\right) x_{l}\right) x_{j} .
$$

Proof. (a) $F_{j}(v)=0$ since $v$ is a braided commutator of two elements in ker $F_{j}$. Using (1-4) we have $\left[\left(\operatorname{ad} x_{k}\right)^{2} x_{j}, x_{j}\right]_{c}=q_{k j}\left(q_{j j}-q_{k k}\right)\left[x_{k} x_{j}\right]_{c}^{2}$, so we calculate

$$
\begin{aligned}
F_{k}(v)= & \left(1+q_{k k}\right)\left(1-q_{k k} q_{k j} q_{j k}\right)\left[x_{k} x_{j}\right]_{c}^{2}-q_{k k}^{2} q_{k j}^{2} q_{j k} q_{j j}\left(1-q_{k j} q_{j k}\right) x_{j}\left[x_{k}^{2} x_{j}\right]_{c} \\
& +q_{k k}^{2} q_{j k}\left(1-q_{k j} q_{j k}\right)\left[x_{k}^{2} x_{j}\right]_{c} x_{j}-q_{k k}^{3} q_{k j}^{2} q_{j k}^{2} q_{j j}\left(1+q_{k k}\right)\left(1-q_{k k} q_{k j} q_{j k}\right)\left[x_{k} x_{j}\right]_{c}^{2} \\
= & q_{k k}^{2} q_{j k} q_{k j}\left(1-q_{k j} q_{j k}\right)\left(q_{j j}-q_{k k}\right) \\
& \quad+\left(1+q_{k k}\right)\left(1-q_{k k} q_{k j} q_{j k}\right)\left(1-q_{k k}^{3} q_{k j}^{2} q_{j k}^{2} q_{j j}\right)\left[x_{k} x_{j}\right]_{c}^{2},
\end{aligned}
$$

which vanishes since the coefficient of $\left[x_{k} x_{j}\right]_{c}^{2}$ is zero for each possible braiding. Thus

$$
\Delta_{1,4}(v)=x_{k} \otimes F_{k}(v)=0 .
$$

Also, $D_{k}(v)=0$, and a calculation gives

$$
\begin{gathered}
D_{j}(v)=\left(1-q_{k j} q_{j k}\right)\left(\left[x_{k}^{2} x_{j}\right] x_{k}+\left(1-q_{k k} q_{k j} q_{j k}\right) q_{j k} q_{j j} x_{k}^{2}\left[x_{k} x_{j}\right]_{c}\right. \\
\left.-q_{k k}^{2} q_{k j}^{2} q_{j k} q_{j j}\left(1-q_{k k} q_{k j} q_{j k}\right)\left[x_{k} x_{j}\right]_{c} x_{k}^{2}-q_{k k}^{2} q_{k j}^{2} q_{j k}^{3} q_{j j}^{2} x_{k}\left[x_{k}^{2} x_{j}\right]\right) \\
=\left(1+\left(1+q_{k k}\right)\left(1-q_{k k} q_{k j} q_{j k}\right) q_{k k} q_{k j} q_{j k} q_{j j}-q_{k k}^{4} q_{k j}^{3} q_{j k}^{3} q_{j j}^{2}\right) \\
\left(1-q_{k j} q_{j k}\right)\left[x_{k}^{2} x_{j}\right] x_{k},
\end{gathered}
$$

where we have reordered the hyperwords and used that $\left(\operatorname{ad} x_{k}\right)^{3} x_{j}=0$; also,

$$
1+\left(1+q_{k k}\right)\left(1-q_{k k} q_{k j} q_{j k}\right) q_{k k} q_{k j} q_{j k} q_{j j}-q_{k k}^{4} q_{k j}^{3} q_{j k}^{3} q_{j j}^{2}=0,
$$

by calculation for each possible braiding. Thus

$$
\Delta_{4,1}(v)=D_{j}(v) \otimes x_{j}=0 .
$$

To finish, we use the fact that $\Delta(v)$ equals

$$
\Delta\left(\left(\operatorname{ad}_{c} x_{k}\right)^{2} x_{j}\right) \Delta\left(\left(\operatorname{ad}_{c} x_{k}\right) x_{j}\right)-\chi\left(2 e_{k}+e_{j}, e_{k}+e_{j}\right) \Delta\left(\left(\operatorname{ad}_{c} x_{k}\right) x_{j}\right) \Delta\left(\left(\operatorname{ad}_{c} x_{k}\right)^{2} x_{j}\right) .
$$

Looking at the terms in $\mathfrak{B}^{3} \otimes \mathfrak{B}^{2}$ and $\mathfrak{B}^{2} \otimes \mathfrak{B}^{3}$, and using the definition of the braided commutator, we obtain

$$
\begin{aligned}
& \Delta_{32}(v) \\
& \begin{array}{r}
=\left(1-q_{k k}^{4} q_{k j}^{3} q_{j k}^{3} q_{j j}^{2}\right)\left[x_{k}^{2} x_{j}\right]_{c} \otimes\left[x_{k} x_{j}\right]_{c} \\
+\left(1+q_{k k}\right)\left(1-q_{k k} q_{k j} q_{j k}\right) q_{k k} q_{k j} q_{j k} q_{j j}\left(x_{k}\left[x_{k} x_{j}\right]_{c}-q_{k k} q_{k j}\left[x_{k} x_{j}\right]_{c} x_{k}\right) \otimes\left[x_{k} x_{j}\right]_{c} \\
+\left(1-q_{k j} q_{j k}\right)^{2}\left(1-q_{k k}^{2} q_{k j} q_{j k}\right)\left(1-q_{k k}^{2} q_{k j}^{2} q_{j k}^{2} q_{j j}\right) x_{k}^{3} \otimes x_{j}^{2}
\end{array} \\
& \begin{array}{r}
\quad\left(1+\left(1+q_{k k}\right)\left(1-q_{k k} q_{k j} q_{j k}\right) q_{k k} q_{k j} q_{j k} q_{j j}-q_{k k}^{4} q_{k j}^{3} q_{j k}^{3} q_{j j}^{2}\right)\left[x_{k}^{2} x_{j}\right]_{c} \otimes\left[x_{k} x_{j}\right]_{c} \\
+b_{1}\left(1-q_{k k}^{2} q_{k j}^{2} q_{j k}^{2} q_{j j}\right) x_{k}^{3} \otimes x_{j}^{2}
\end{array}
\end{aligned}
$$


Also,

$$
\begin{aligned}
\Delta_{23}(v)=\left(1-q_{k k} q_{k j} q_{j k}\right)\left(1-q_{k j} q_{j k}\right) x_{k}^{2} & \\
\otimes\left(\left(1+q_{k k}\right) q_{k k} q_{j k}\left[x_{k} x_{j}\right]_{c} x_{j}-\right. & \left(1+q_{k k}\right) q_{k k}^{2} q_{k j}^{2} q_{j k}^{2} q_{j j} x_{j}\left[x_{k} x_{j}\right]_{c} \\
& \left.+x_{j}\left[x_{k} x_{j}\right]_{c}-q_{k k}^{4} q_{k j}^{2} q_{j k}^{3} q_{j j}\left[x_{k} x_{j}\right]_{c} x_{j}\right) \\
=\left(1-q_{k k}^{4} q_{k j}^{3} q_{j k}^{3} q_{j j}^{2}+\left(1+q_{k k}\right)\right. & \left.\left(1-q_{k k} q_{k j} q_{j k}\right) q_{k k} q_{k j} q_{j k} q_{j j}\right) \\
& \left(1-q_{k k} q_{k j} q_{j k}\right)\left(1-q_{k j} q_{j k}\right) x_{k}^{2} \otimes x_{j}\left[x_{k} x_{j}\right]_{c} .
\end{aligned}
$$

Using (5-12), we obtain (5-10).

(b) We set $y=\left(\operatorname{ad} x_{k}\right)^{2}\left(\operatorname{ad} x_{j}\right) x_{l}$ and $z=\left(\operatorname{ad} x_{k}\right) x_{j}$. Note that $\Delta(w)=\Delta(y) \Delta(z)-$ $\chi\left(2 \mathbf{e}_{k}+\mathbf{e}_{j}+\mathbf{e}_{l}, \mathbf{e}_{k}+\mathbf{e}_{j}\right) \Delta(z) \Delta(y)$ and that

$$
\begin{aligned}
\Delta(y)=y \otimes 1+\left(1-q_{j l} q_{l j}\right)\left(\operatorname{ad} x_{k}\right)^{2} x_{j} \otimes x_{l} \\
+\left(1-q_{k j} q_{j k}\right)\left(1-q_{k k} q_{k j} q_{j k}\right) x_{k}^{2} \otimes\left(\operatorname{ad} x_{j}\right) x_{l} \\
\quad+\left(1+q_{k k}\right)\left(1-q_{k k} q_{k j} q_{j k}\right) x_{k} \otimes\left(\operatorname{ad} x_{k}\right)\left(\operatorname{ad} x_{j}\right) x_{l}+1 \otimes y,
\end{aligned}
$$

$\Delta(z)=z \otimes 1+\left(1-q_{k j} q_{j k}\right) x_{k} \otimes x_{j}+1 \otimes z$.

From (2-3) we have $D_{k}(w)=0$, and from (2-4),

$$
\begin{aligned}
D_{l}(w) & =\left(1-q_{l j} q_{j l}\right) q_{l k} q_{l j}\left[\left(\operatorname{ad} x_{k}\right)^{2} x_{j},\left(\operatorname{ad} x_{k}\right) x_{j}\right]_{c}, \\
D_{j}(w) & =-\left(1-q_{k j} q_{j k}\right) q_{k k}^{-2} q_{k j}^{-1} q_{k l}^{-1}\left(\operatorname{ad} x_{k}\right)^{3}\left(\operatorname{ad} x_{j}\right) x_{l} \\
& =-\left(1-q_{k j} q_{j k}\right) q_{k k}^{-2} q_{k j}^{-1} q_{k l}^{-1}\left[\left(\operatorname{ad} x_{k}\right)^{3} x_{j}, x_{l}\right]_{c}=0,
\end{aligned}
$$

where in the last equality we used (1-4) and the vanishing of $\left[x_{k}, x_{l}\right]_{c}=0$. It follows that

$$
\Delta_{51}(w)=\left(1-q_{l j} q_{j l}\right) q_{l k} q_{l j}\left[\left(\operatorname{ad} x_{k}\right)^{2} x_{j},\left(\operatorname{ad} x_{k}\right) x_{j}\right]_{c} \otimes x_{l} .
$$

Also, $F_{j}(z)=F_{j}(y)=F_{l}(z)=F_{l}(y)=0$ by $(2-6)$ and the properties of these skew derivations, so $F_{j}(w)=F_{l}(w)=0$. We now calculate $F_{k}(w)$

$=\left(1+q_{k k}\right)\left(1-q_{k k} q_{k j} q_{j k}\right)\left[x_{k} x_{j} x_{l}\right]_{c}\left[x_{k} x_{j}\right]_{c}+q_{k k}^{2} q_{j k} q_{l k}\left(1-q_{k j} q_{j k}\right)\left[x_{k}^{2} x_{j} x_{l}\right]_{c} x_{j}$

$$
-\chi\left(2 \mathbf{e}_{k}+\mathbf{e}_{j}+\mathbf{e}_{l}, \mathbf{e}_{k}+\mathbf{e}_{j}\right)
$$

$\left(\left(1-q_{k j} q_{j k}\right) x_{j}\left[x_{k}^{2} x_{j} x_{l}\right]_{c}+\left(1+q_{k k}\right)\left(1-q_{k k} q_{k j} q_{j k}\right) q_{k k} q_{j k}\left[x_{k} x_{j} x_{l}\right]_{c}\left[x_{k} x_{j}\right]_{c}\right)$

$=q_{k k}^{2} q_{j k} q_{l k}\left(1-q_{k j} q_{j k}\right)\left[\left[x_{k}^{2} x_{j} x_{l}\right]_{c}, x_{j}\right]_{c}$

$-\left(1+q_{k k}\right)\left(1-q_{k k} q_{k j} q_{j k}\right) q_{k k}^{3} q_{k j}^{2} q_{j k}^{2} q_{j j} q_{l j} q_{l k}\left[\left[x_{k} x_{j}\right]_{c},\left[x_{k} x_{j} x_{l}\right]_{c}\right]_{c}$

$=q_{k k}^{2} q_{k j} q_{j k} q_{j j} q_{l j} q_{l k}$

$\left(1-q_{k j} q_{j k}-\left(1+q_{k k}\right)\left(1-q_{k k} q_{k j} q_{j k}\right) q_{k k} q_{k j} q_{j k}\right)\left[\left[x_{k} x_{j}\right]_{c},\left[x_{k} x_{j} x_{l}\right]_{c}\right]_{c}$ $=0$, 
where we used (1-4) and (5-4) in the third equality, and we calculate that

$$
1-q_{k j} q_{j k}-\left(1+q_{k k}\right)\left(1-q_{k k} q_{k j} q_{j k}\right) q_{k k} q_{k j} q_{j k}=0
$$

for each possible standard braiding. It follows that $\Delta_{15}(w)=0$.

We find each of the other terms of $\Delta(w)$ by direct calculation. First,

$$
\begin{aligned}
& \Delta_{42}(w) \\
&=\left(1-\chi\left(2 \mathbf{e}_{k}+\mathbf{e}_{j}+\mathbf{e}_{l}, \mathbf{e}_{k}+\mathbf{e}_{j}\right) \chi\left(\mathbf{e}_{k}+\mathbf{e}_{j}, 2 \mathbf{e}_{k}+\mathbf{e}_{j}+\mathbf{e}_{l}\right)\right) y \otimes z \\
&+\left(1-q_{k j} q_{j k}\right)\left(1-q_{l j} q_{j l}\right) \\
& \quad\left(q_{l k}\left[x_{k}^{2} x_{j}\right]_{c} x_{k} \otimes x_{l} x_{j}-\chi\left(2 \mathbf{e}_{k}+\mathbf{e}_{j}+\mathbf{e}_{l}, \mathbf{e}_{k}+\mathbf{e}_{j}\right) q_{j k}^{2} q_{j j} x_{k}\left[x_{k}^{2} x_{j}\right]_{c} \otimes x_{j} x_{l}\right) \\
&+\left(1-q_{k j} q_{j k}\right)\left(1-q_{k k} q_{k j} q_{j k}\right) \\
& \quad\left(\chi\left(\mathbf{e}_{j}+\mathbf{e}_{l}, \mathbf{e}_{k}+\mathbf{e}_{j}\right) x_{k}^{2} z-\chi\left(2 \mathbf{e}_{k}+\mathbf{e}_{j}+\mathbf{e}_{l}, \mathbf{e}_{k}+\mathbf{e}_{j}\right) z x_{k}^{2}\right) \otimes\left[x_{j} x_{l}\right]_{c} \\
&=\left(1-q_{k j} q_{j k}\right) q_{l k}\left(1-q_{j k} q_{k j}+\left(1+q_{k k}\right)\left(1-q_{k k} q_{k j} q_{j k}\right) q_{k k} q_{k j} q_{j k}\right) \\
& {\left[x_{k}^{2} x_{j}\right]_{c} x_{k} \otimes\left[x_{j} x_{l}\right]_{c}, }
\end{aligned}
$$

which is seen to equal 0 . In a similar way we calculate

$$
\begin{aligned}
\Delta_{33}(w) & \\
= & \left(1-q_{l j} q_{j l}\right)\left[x_{k}^{2} x_{j}\right] \otimes\left(x_{l} z-\chi\left(2 \mathbf{e}_{k}+\mathbf{e}_{j}+\mathbf{e}_{l}, \mathbf{e}_{k}+\mathbf{e}_{j}\right) \chi\left(\mathbf{e}_{k}+\mathbf{e}_{j}, \mathbf{e}_{k}+\mathbf{e}_{j}+\mathbf{e}_{l}\right) z x_{l}\right) \\
& +\left(1+q_{k k}\right)\left(1-q_{k k} q_{k j} q_{j k}\right) \chi\left(\mathbf{e}_{k}+\mathbf{e}_{j}+\mathbf{e}_{l}, \mathbf{e}_{k}+\mathbf{e}_{j}\right)\left(x_{k} z-q_{k k} q_{k j} z x_{k}\right) \otimes\left[x_{k} x_{j} x_{l}\right]_{c} \\
& +\left(1-q_{k k} q_{k j} q_{j k}\right)\left(1-q_{k j} q_{j k}\right)^{2} x_{k}^{3} \\
& \otimes\left(\chi\left(\mathbf{e}_{j}+\mathbf{e}_{l}, \mathbf{e}_{k}\right)\left[x_{j} x_{l}\right]_{c} x_{j}-\chi\left(2 \mathbf{e}_{k}+\mathbf{e}_{j}+\mathbf{e}_{l}, \mathbf{e}_{k}+\mathbf{e}_{j}\right) \chi\left(\mathbf{e}_{j}, 2 \mathbf{e}_{k}\right) x_{j}\left[x_{j} x_{l}\right]_{c}\right) \\
= & \left(\left(1+q_{k k}\right)\left(1-q_{k k} q_{k j} q_{j k}\right)-q_{k k} q_{k j} q_{j k} q_{j j}\left(1-q_{l j} q_{j l}\right)\right) \\
& \chi\left(\mathbf{e}_{k}+\mathbf{e}_{j}+\mathbf{e}_{l}, \mathbf{e}_{k}+\mathbf{e}_{j}\right)\left[x_{k}^{2} x_{j}\right]_{c} \otimes\left[x_{k} x_{j} x_{l}\right]_{c} \\
& +\left(1-q_{k k} q_{k j} q_{j k}\right)\left(1-q_{k j} q_{j k}\right)^{2}\left(1-q_{k k}^{2} q_{k j} q_{j k}\right) x_{k}^{3} \otimes\left[x_{j} x_{l}\right]_{c} x_{j},
\end{aligned}
$$

and the coefficient of $\left[x_{k}^{2} x_{j}\right]_{c} \otimes\left[x_{k} x_{j} x_{l}\right]_{c}$ is zero (we calculate it for each possible standard braiding). Finally,

$\Delta_{24}(w)$

$$
\begin{aligned}
& =\left(1-q_{k k} q_{k j} q_{j k}\right)\left(1-q_{k j} q_{j k}\right) x_{k}^{2} \\
& \otimes\left(\left(1+q_{k k}\right) \chi\left(\mathbf{e}_{k}+\mathbf{e}_{j}+\mathbf{e}_{l}, \mathbf{e}_{k}\right)\left[x_{k} x_{j} x_{l}\right]_{c} x_{j}\right. \\
& -\left(1+q_{k k}\right) \chi\left(2 \mathbf{e}_{k}+\mathbf{e}_{j}+\mathbf{e}_{l}, \mathbf{e}_{k}+\mathbf{e}_{j}\right) q_{j k} x_{j}\left[x_{k} x_{j} x_{l}\right]_{c} \\
& \left.-\chi\left(2 \mathbf{e}_{k}+\mathbf{e}_{j}+\mathbf{e}_{l}, \mathbf{e}_{k}+\mathbf{e}_{j}\right) \chi\left(\mathbf{e}_{k}+\mathbf{e}_{j}, 2 \mathbf{e}_{k}\right)\left[\left[x_{k} x_{j}\right]_{c},\left[x_{j} x_{l}\right]_{c}\right]_{c}\right) \\
& =\left(1-q_{k k} q_{k j} q_{j k}\right)\left(1-q_{k j} q_{j k}\right) \chi\left(\mathbf{e}_{j}+\mathbf{e}_{l}, \mathbf{e}_{k}+\mathbf{e}_{j}\right) q_{k j} \\
& \left(q_{k k}\left(1-q_{k k} q_{k j} q_{j k}\right)-q_{j j}\left(1-q_{j l} q_{l j}\right)\right) x_{k}^{2} \otimes x_{j}\left[x_{k} x_{j} x_{l}\right]_{c} \\
& =0 \text {. }
\end{aligned}
$$

From these calculations, we obtain (5-11). 
5B. Presentation when the type is $\boldsymbol{A}_{\theta}$. We now assume $V$ is a standard braided vector space of type $A_{\theta}$ and $\mathfrak{B}$ a $\mathbb{Z}^{\theta}$-graded algebra, provided with an inclusion of vector spaces $V \hookrightarrow \mathfrak{B}^{1}=\bigoplus_{1 \leq j \leq \theta} \mathfrak{B}^{\mathbf{e}_{j}}$. We can extend the braiding to $\mathfrak{B}$ by setting

$$
c(u \otimes v)=\chi(\alpha, \beta) v \otimes u, \quad u \in \mathfrak{B}^{\alpha}, v \in \mathfrak{B}^{\beta}, \alpha, \beta \in \mathbb{N}^{\theta} .
$$

We assume that on $\mathfrak{B}$ we have

$$
\begin{aligned}
& x_{i}^{2}=0 \text { if } q_{i i}=-1, \\
& \operatorname{ad}_{c} x_{i}\left(x_{j}\right)=0 \text { if }|j-i|>1, \\
& \left(\operatorname{ad}_{c} x_{i}\right)^{2}\left(x_{j}\right)_{c}=0 \text { if }|j-i|=1 \text {, } \\
& {\left[\left(\operatorname{ad}_{c} x_{i}\right)\left(\operatorname{ad}_{c} x_{i+1}\right) x_{i+2}, x_{i+1}\right]_{c}=0 \quad 2 \leq i \leq \theta-1 .}
\end{aligned}
$$

Using the same notation as in Section $4 \mathrm{~B}$,

$$
x_{\mathbf{e}_{i}}=x_{i}, \quad x_{\mathbf{u}_{i, j}}:=\left[x_{i}, x_{\mathbf{u}_{i+1, j}}\right]_{c} \quad(i<j) .
$$

Lemma 5.10. Let $1 \leq i \leq j<p \leq r \leq \theta$. The following relations hold in $\mathfrak{B}$ :

$$
\begin{aligned}
{\left[x_{\mathbf{u}_{i j}}, x_{\mathbf{u}_{p r}}\right]_{c} } & =0, p-j \geq 2 ; \\
{\left[x_{\mathbf{u}_{i j}}, x_{\mathbf{u}_{j+1, r}}\right]_{c} } & =x_{\mathbf{u}_{i r}} .
\end{aligned}
$$

Proof. Note that $x_{\mathbf{u}_{p r}}$ belongs to the subalgebra generated by $x_{p}, \ldots, x_{r}$, and $\left[x_{\mathbf{u}_{i j}}, x_{s}\right]_{c}=0$, for each $p \leq s \leq r$. Equation (5-14) follows from this.

We prove (5-15) by induction on $j-i$ : if $i=j$, it is exactly the definition of $x_{\mathbf{u}_{i r}}$. To prove the inductive step, we use the inductive hypothesis, (5-14) and (1-4) (the braided Jacobi identity) to obtain

$$
\begin{aligned}
{\left[x_{\mathbf{u}_{i, j+1}}, x_{\mathbf{u}_{j+2, r}}\right]_{c} } & =\left[\left[x_{\mathbf{u}_{i j}}, x_{i+1}\right]_{c}, x_{\mathbf{u}_{j+2, r}}\right]_{c}=\left[x_{\mathbf{u}_{i j}},\left[x_{i+1}, x_{\mathbf{u}_{j+2, r}}\right]_{c}\right]_{c} \\
& =\left[x_{\mathbf{u}_{i j}}, x_{\mathbf{u}_{j+1, r}}\right]_{c}=x_{\mathbf{u}_{i r}},
\end{aligned}
$$

and (5-15) is also proved.

Lemma 5.11. If $i<p \leq r<j$, the following relation holds in $\mathfrak{B}$ :

$$
\left[x_{\mathbf{u}_{i j}}, x_{\mathbf{u}_{p r}}\right]_{c}=0 .
$$

Proof. When $p=r=j-1$ and $i=j-2$, note that this is exactly

$$
\left[\left(\operatorname{ad}_{c} x_{i}\right)\left(\operatorname{ad}_{c} x_{i+1}\right) x_{i+2}, x_{i+1}\right]_{c}=0 \text {. }
$$

Then, by (1-4),

$$
\left[x_{\mathbf{u}_{i-1, j}}, x_{j-1}\right]_{c}=\left[\left[x_{i-1}, x_{\mathbf{u}_{i, j}}\right]_{c}, x_{j-1}\right]_{c}=\left[x_{i-1},\left[x_{\mathbf{u}_{i, j}}, x_{j-1}\right]_{c}\right]_{c} .
$$

We assume that $j-i>2$, so $\left[x_{i-1}, x_{j-1}\right]_{c}=0$ by the hypothesis on $\mathfrak{B}$. Now we prove the case $p=r=j-1$ by induction on $p-i$. 
Using (1-4) and (5-15), we also have

$$
\begin{aligned}
{\left[x_{\mathbf{u}_{i, j+1}}, x_{p}\right]_{c}=} & {\left[\left[x_{\mathbf{u}_{i, j}}, x_{j+1}\right]_{c}, x_{p}\right]_{c}=\left[x_{\mathbf{u}_{i, j}},\left[x_{j+1}, x_{p}\right]_{c}\right]_{c} } \\
& +q_{j+1, p}\left[x_{\mathbf{u}_{i, j}}, x_{j-1}\right]_{c} x_{j+1}-\chi\left(\mathbf{u}_{i, j}, \mathbf{e}_{j+1}\right) x_{j+1}\left[x_{\mathbf{u}_{i, j}}, x_{j-1}\right]_{c},
\end{aligned}
$$

so using that $\left[x_{j+1}, x_{p}\right]_{c}=0$ if $j>p$, by induction on $j-p$ we prove (5-16) for the case $p=r$.

For the general case, we use (1-4) one more time as follows

$$
\begin{aligned}
{\left[x_{\mathbf{u}_{i, j}}, x_{\mathbf{u}_{p, r+1}}\right]_{c} } & =\left[x_{\mathbf{u}_{i, j}},\left[x_{\mathbf{u}_{p r}}, x_{r+1}\right]_{c}\right]_{c}=\left[\left[x_{\mathbf{u}_{i, j}}, x_{\mathbf{u}_{p r}}\right]_{c}, x_{r+1}\right]_{c} \\
& -\chi\left(\mathbf{u}_{p r}, \mathbf{e}_{r+1}\right)\left[x_{\mathbf{u}_{i j}}, x_{r+1}\right]_{c} x_{\mathbf{u}_{p r}}+\chi\left(\mathbf{u}_{i j}, \mathbf{u}_{p r}\right) x_{\mathbf{u}_{p r}}\left[x_{\mathbf{u}_{i j}}, x_{r+1}\right]_{c},
\end{aligned}
$$

and we prove (5-16) by induction on $r-p$.

Lemma 5.12. The following relations hold in $\mathfrak{B}$ :

$$
\begin{array}{cc}
{\left[x_{\mathbf{u}_{i j}}, x_{\mathbf{u}_{i p}}\right]_{c}=0} & \text { if } i \leq j<p, \\
{\left[x_{\mathbf{u}_{i j}}, x_{\mathbf{u}_{p j}}\right]_{c}=0} & \text { if } i<p \leq j .
\end{array}
$$

Proof. To prove (5-17), note that if $i=j=p-1$, we have

$$
\left[x_{\mathbf{u}_{i i}}, x_{\mathbf{u}_{i, i+1}}\right]_{c}=\left[x_{i},\left[x_{i}, x_{i+1}\right]_{c}\right]_{c}=\left(\operatorname{ad} x_{i}\right)^{2} x_{i+1}=0 .
$$

Since $\left[x_{i}, x_{\mathbf{u}_{i+2, p}}\right]_{c}=0$ for each $p>i+1$ by (5-14), we use (1-4), the previous case and (5-15) to obtain

$$
\left[x_{\mathbf{u}_{i i}}, x_{\mathbf{u}_{i p}}\right]_{c}=\left[x_{\mathbf{u}_{i i}},\left[x_{\mathbf{u}_{i, i+1}}, x_{\mathbf{u}_{i+2, p}}\right]_{c}\right]_{c}=0 .
$$

Now, if $i<j<p$, from (5-14) and the relations between the $q_{s t}$ we obtain

$$
\left[x_{\mathbf{u}_{i+1, j}}, x_{\mathbf{u}_{i p}}\right]_{c}=-\chi\left(\mathbf{u}_{i p}, \mathbf{u}_{i+1, j}\right)\left[x_{\mathbf{u}_{i p}}, x_{\mathbf{u}_{i+1, j}}\right]_{c}=0 .
$$

Using (1-4) and the previous case we conclude

$$
\left[x_{\mathbf{u}_{i j}}, x_{\mathbf{u}_{i p}}\right]_{c}=\left[\left[x_{\mathbf{u}_{i i}}, x_{\mathbf{u}_{i+1, j}}\right]_{c}, x_{\mathbf{u}_{i p}}\right]_{c}=0 .
$$

The proof of (5-18) is analogous.

Lemma 5.13. If $i<p \leq r<j$, the following relation holds in $\mathfrak{B}$ :

$$
\left[x_{\mathbf{u}_{i r}}, x_{\mathbf{u}_{p j}}\right]_{c}=\chi\left(\mathbf{u}_{i r}, \mathbf{u}_{p r}\right)\left(1-q_{r, r+1} q_{r+1, r}\right) x_{\mathbf{u}_{p r}} x_{\mathbf{u}_{i j}} .
$$

Proof. We calculate

$$
\begin{aligned}
{\left[x_{\mathbf{u}_{i r}}, x_{\mathbf{u}_{p j}}\right]_{c} } & =\left[x_{\mathbf{u}_{i r}},\left[x_{\mathbf{u}_{p r}}, x_{\mathbf{u}_{r+1, j}}\right]_{c}\right]_{c} \\
& =\chi\left(\mathbf{u}_{i r}, \mathbf{u}_{p r}\right) x_{\mathbf{u}_{p r}} x_{\mathbf{u}_{i j}}-\chi\left(\mathbf{u}_{p r}, \mathbf{u}_{r+1, j}\right) x_{\mathbf{u}_{i j}} x_{\mathbf{u}_{p r}} \\
& =\left(\chi\left(\mathbf{u}_{i r}, \mathbf{u}_{p r}\right)-\chi\left(\mathbf{u}_{i j}, \mathbf{u}_{p r}\right) \chi\left(\mathbf{u}_{p r}, \mathbf{u}_{r+1, j}\right)\right) x_{\mathbf{u}_{p r}} x_{\mathbf{u}_{i j}} \\
& =\chi\left(\mathbf{u}_{i r}, \mathbf{u}_{p r}\right)\left(1-\chi\left(\mathbf{u}_{p r}, \mathbf{u}_{r+1, j}\right) \chi\left(\mathbf{u}_{r+1, j}, \mathbf{u}_{p r}\right)\right) x_{\mathbf{u}_{p r}} x_{\mathbf{u}_{i j}}
\end{aligned}
$$


where we have used (5-15) in the first equality, (1-4) in the second, (5-18) in the third and the relation between the $q_{i j}$ in the last.

We now prove the main theorem of this subsection, namely, the presentation by generators and relations of the Nichols algebra associated to $V$.

Theorem 5.14. Let $V$ be a standard braided vector space of type $A_{\theta}$, where $\theta=$ $\operatorname{dim} V$, and let $C=\left(a_{i j}\right)_{i, j \in\{1, \ldots, \theta\}}$ be the corresponding Cartan matrix of type $A_{\theta}$.

The Nichols algebra $\mathfrak{B}(V)$ is presented by the generators $x_{i}, 1 \leq i \leq \theta$, and the relations

$$
\begin{aligned}
x_{\alpha}^{N_{\alpha}} & =0, & & \alpha \in \Delta^{+} ; \\
\operatorname{ad}_{c}\left(x_{i}\right)^{1-a_{i j}}\left(x_{j}\right) & =0, & & i \neq j ; \\
{\left[\left(\operatorname{ad} x_{j-1}\right)\left(\operatorname{ad} x_{j}\right) x_{j+1}, x_{j}\right]_{c} } & =0, & & 1<j<\theta, q_{j j}=-1 .
\end{aligned}
$$

The following elements constitute a basis of $\mathfrak{B}(V)$ :

$x_{\beta_{1}}^{h_{1}} x_{\beta_{2}}^{h_{2}} \ldots x_{\beta_{P}}^{h_{P}}$, where $0 \leq h_{j}<N_{\beta_{j}}$ where $\beta_{j} \in S_{I}$, for $1 \leq j \leq P$.

Proof. From Corollary 4.2 and the definitions of the $x_{\alpha}$, we know that the last statement about the PBW basis is true.

Let $\mathfrak{B}$ be the algebra presented by generators $x_{1}, \ldots, x_{\theta}$ and the relations in the statement of the theorem. From Lemmas 5.3, 5.4 and Corollary 5.2 we have a canonical epimorphism $\phi: \mathfrak{B} \rightarrow \mathfrak{B}(V)$. The last relation also holds in $\mathfrak{B}$ for $q_{j j} \neq 1$, by Lemma 5.4(2).

The rest is similar to the proofs of [Andruskiewitsch and Dăscălescu 2005, Lemma 3.7] and [Andruskiewitsch and Schneider 2002b, Lemma 6.12]. Consider the subspace $\mathscr{I}$ of $\mathfrak{B}$ generated by the elements in (5-20). Using Lemmas 5.10, 5.11, 5.12 and 5.13 we prove that $\mathscr{I}$ is an ideal. But $1 \in \mathscr{I}$, so $\mathscr{I}=\mathfrak{B}$.

The images under $\phi$ of the elements in (5-20) form a basis of $\mathfrak{B}(V)$, so $\phi$ is an isomorphism.

The presentation and dimension of $\mathfrak{B}(V)$ agree with the results presented in [Andruskiewitsch and Dăscălescu 2005] and [Andruskiewitsch and Schneider 2002b].

5C. Presentation when the type is $\boldsymbol{B}_{\boldsymbol{\theta}}$. We now assume $V$ is a standard braided vector space of type $B_{\theta}$ and $\mathfrak{B}$ is a $\mathbb{Z}^{\theta}$-graded algebra, provided with an inclusion of vector spaces $V \hookrightarrow \mathfrak{B}^{1}=\bigoplus_{1 \leq j \leq \theta} \mathfrak{B}^{\mathbf{e}_{j}}$. Then we can extend the braiding to $\mathfrak{B}$. We assume the following relations in $\mathfrak{B}$ :

$$
\begin{aligned}
x_{i}^{2}=0 & \text { if } q_{i i}=-1, \\
x_{1}^{3}=0 & \text { if } q_{11} \in \mathbb{G}_{3}, \\
\left(\operatorname{ad}_{c} x_{i}\right) x_{j}=0 & \text { if }|j-i|>1, \\
\left(\operatorname{ad}_{c} x_{i}\right)^{2} x_{j}=0 & \text { if }|j-i|=1 \text { and } i \neq 1,
\end{aligned}
$$




$$
\begin{aligned}
{\left[\left(\operatorname{ad}_{c} x_{i}\right)\left(\operatorname{ad}_{c} x_{i+1}\right) x_{i+2}, x_{i+1}\right]_{c} } & =0 \quad \text { if } 2 \leq i \leq \theta, \\
\left(\operatorname{ad}_{c} x_{1}\right)^{3} x_{2} & =0, \\
{\left[\left(\operatorname{ad}_{c} x_{1}\right)^{2} x_{2},\left(\operatorname{ad}_{c} x_{1}\right) x_{2}\right]_{c} } & =0, \\
{\left[\left(\operatorname{ad} x_{1}\right)^{2}\left(\operatorname{ad} x_{2}\right) x_{3},\left(\operatorname{ad} x_{1}\right) x_{2}\right]_{c} } & =0 .
\end{aligned}
$$

Using the same notation as in Section 4B,

$$
x_{\mathbf{v}_{i j}}=\left[x_{\mathbf{u}_{1 i}}, x_{\mathbf{u}_{1 j}}\right]_{c}, \quad 1 \leq i<j \leq \theta .
$$

From the proof of the relations corresponding the $A_{\theta}$ case, we know that (5-14), (5-15), (5-16), (5-18) and (5-19) hold for $i \geq 1$, but for relation (5-17) we must assume $i>1$.

Lemma 5.15. Suppose $1 \leq s<t$ and $1<k \leq j$. The following relations hold in $\mathfrak{B}$ :

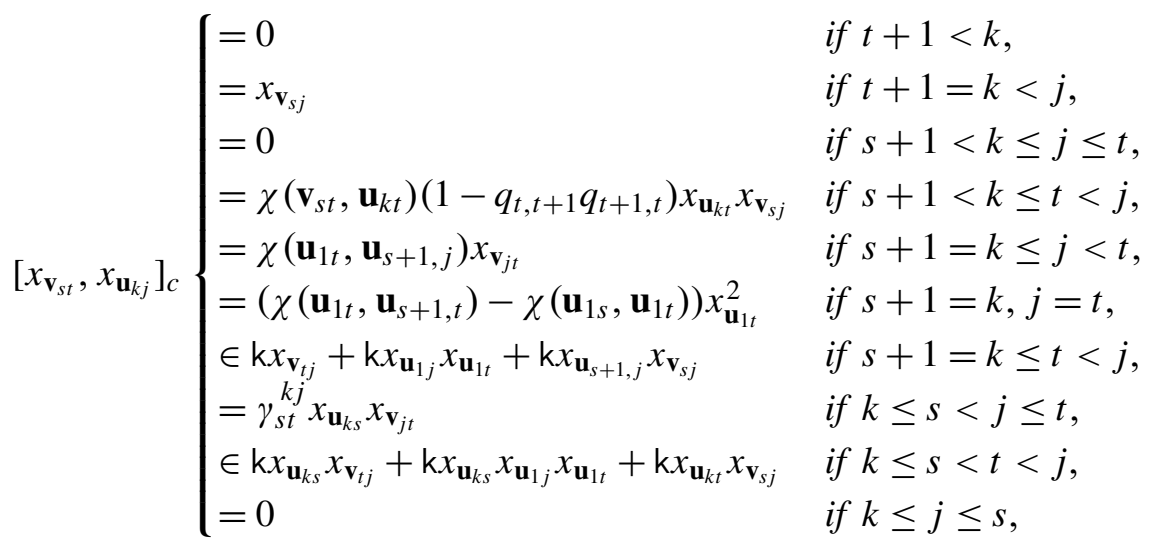

where $\gamma_{s t}^{k j}=\chi\left(\mathbf{u}_{1 t}, \mathbf{u}_{k j}\right) \chi\left(\mathbf{u}_{1 s}, \mathbf{u}_{k s}\right)\left(1-q_{s, s+1} q_{s+1, s}\right)$.

Proof. The first, third and last equalities follow from the vanishing of $\left[x_{\mathbf{u}_{1 s}}, x_{\mathbf{u}_{k j}}\right]_{c}$ and $\left[x_{\mathbf{u}_{1 t}}, x_{\mathbf{u}_{k j}}\right]_{c}=0$, using (5-14), (5-16), (5-17) or (5-18) as the case maybe, together with (1-4).

For the second case, we use that $\left[x_{\mathbf{u}_{1 s}}, x_{\mathbf{u}_{t+1, j}}\right]_{c}=0,(5-15)$ and (1-4) to obtain $x_{\mathbf{v}_{s j}}=\left[x_{\mathbf{u}_{1 s}}, x_{\mathbf{u}_{1 j}}\right]_{c}=\left[x_{\mathbf{u}_{1 s}},\left[x_{\mathbf{u}_{1 t}}, x_{\mathbf{u}_{t+1, j}}\right]_{c}\right]_{c}=\left[\left[x_{\mathbf{u}_{1 s}}, x_{\mathbf{u}_{1 t}}\right]_{c}, x_{\mathbf{u}_{t+1, j}}\right]_{c}=\left[x_{\mathbf{v}_{s t}}, x_{\mathbf{u}_{t+1, j}}\right]_{c}$.

For the fourth, we use (1-4) and the third case to calculate

$$
\begin{aligned}
{\left[x_{\mathbf{v}_{s t}}, x_{\mathbf{u}_{k j}}\right]_{c} } & =\left[x_{\mathbf{v}_{s t}},\left[x_{\mathbf{u}_{k t}}, x_{\mathbf{u}_{t+1, j}}\right]_{c}\right]_{c} \\
= & \chi\left(\mathbf{v}_{s t}, \mathbf{u}_{k t}\right) x_{\mathbf{u}_{k t}} x_{\mathbf{v}_{s j}}-\chi\left(\mathbf{u}_{k t}, \mathbf{u}_{t+1, j}\right) x_{\mathbf{v}_{s j}} x_{\mathbf{u}_{k t}} \\
& =\chi\left(\mathbf{v}_{s t}, \mathbf{u}_{k t}\right)\left(1-\chi\left(\mathbf{u}_{k t}, \mathbf{u}_{t+1, j}\right) \chi\left(\mathbf{u}_{t+1, j}, \mathbf{u}_{k t}\right)\right) x_{\mathbf{u}_{k t}} x_{\mathbf{v}_{s j}} .
\end{aligned}
$$


For the fifth, note that $\chi\left(\mathbf{u}_{1 t}, \mathbf{u}_{s+1, j}\right)^{-1}=\chi\left(\mathbf{u}_{s+1, j}, \mathbf{u}_{1 t}\right)$. Then use (5-15), (5-16) and (1-4) to prove that

$$
\begin{aligned}
{\left[x_{\mathbf{v}_{s t}}, x_{\mathbf{u}_{s+1, j}}\right]_{c} } & =\left[\left[x_{\mathbf{u}_{1 s}}, x_{\mathbf{u}_{1 t}}\right]_{c}, x_{\mathbf{u}_{s+1, j}}\right]_{c} \\
& =\chi\left(\mathbf{u}_{1 t}, \mathbf{u}_{s+1, j}\right) x_{\mathbf{u}_{1 j}} x_{\mathbf{u}_{1 t}}-\chi\left(\mathbf{u}_{1 s}, \mathbf{u}_{1 t}\right) x_{\mathbf{u}_{1 t}} x_{\mathbf{u}_{1 s}} \\
& =\chi\left(\mathbf{u}_{1 t}, \mathbf{u}_{s+1, j}\right)\left(x_{\mathbf{u}_{1 j}} x_{\mathbf{u}_{1 t}}-\chi\left(\mathbf{u}_{1 j}, \mathbf{u}_{1 t}\right) x_{\mathbf{u}_{1 t}} x_{\mathbf{u}_{1 s}}\right) .
\end{aligned}
$$

The sixth case is similar.

For the seventh case, we use (1-4), (1-5) and the previous case to calculate

$$
\begin{aligned}
& {\left[x_{\mathbf{v}_{s t}}, x_{\mathbf{u}_{s+1, j}}\right]_{c}=\left[x_{\mathbf{v}_{s t}},\left[x_{\mathbf{u}_{s+1, t}}, x_{\mathbf{u}_{t+1, j}}\right]_{c}\right]_{c}} \\
& =\left(\chi\left(\mathbf{u}_{1 t}, \mathbf{u}_{s+1, t}\right)-\chi\left(\mathbf{u}_{1 s}, \mathbf{u}_{1 t}\right)\right)\left[x_{\mathbf{u}_{1 t}}^{2}, x_{\mathbf{u}_{t+1, j}}\right] \\
& +\chi\left(\mathbf{v}_{s t}, \mathbf{u}_{s+1, t}\right) x_{\mathbf{u}_{s+1, t}} x_{\mathbf{v}_{s j}}-\chi\left(\mathbf{u}_{s+1, t}, \mathbf{u}_{t+1, j}\right) x_{\mathbf{v}_{s j}} x_{\mathbf{u}_{s+1, t}} \\
& =\left(\chi\left(\mathbf{u}_{1 t}, \mathbf{u}_{s+1, t}\right)-\chi\left(\mathbf{u}_{1 s}, \mathbf{u}_{1 t}\right)\right)\left(\left(x_{\mathbf{v}_{t j}}+\chi\left(\mathbf{u}_{1 t}, \mathbf{u}_{1 j}\right) x_{\mathbf{u}_{1 j}} x_{\mathbf{u}_{1 t}}\right)\right. \\
& \left.+\chi\left(\mathbf{u}_{1 t}, \mathbf{u}_{t+1, j}\right) x_{\mathbf{u}_{1 j}} x_{\mathbf{u}_{1 t}}\right)-\chi\left(\mathbf{u}_{s+1, t}, \mathbf{u}_{t+1, j}\right) x_{\mathbf{v}_{t j}} \\
& +\left(\chi\left(\mathbf{v}_{s t}, \mathbf{u}_{s+1, t}\right)-\chi\left(\mathbf{u}_{s+1, t}, \mathbf{u}_{t+1, j}\right) \chi\left(\mathbf{v}_{s j}, \mathbf{u}_{s+1, t}\right)\right) x_{\mathbf{u}_{s+1, t}} x_{\mathbf{v}_{s j}} .
\end{aligned}
$$

We use the previous cases, (5-16) and (5-19) to calculate for the eighth case

$$
\begin{aligned}
{\left[x_{\mathbf{v}_{s t}}, x_{\mathbf{u}_{k j}}\right]_{c}=} & {\left[\left[x_{\mathbf{u}_{1 s}}, x_{\mathbf{u}_{1 t}}\right]_{c}, x_{\mathbf{u}_{k j}}\right]_{c} } \\
= & \chi\left(\mathbf{u}_{1 t}, \mathbf{u}_{k j}\right)\left(\chi\left(\mathbf{u}_{1 s}, \mathbf{u}_{k s}\right)\left(1-q_{s, s+1} q_{s+1, s}\right) x_{\mathbf{u}_{k s}} x_{\mathbf{u}_{1 j}}\right) x_{\mathbf{u}_{1 t}} \\
& \quad-\chi\left(\mathbf{u}_{1 s}, \mathbf{u}_{1 t}\right) x_{\mathbf{u}_{1 t}}\left(\chi\left(\mathbf{u}_{1 s}, \mathbf{u}_{k s}\right)\left(1-q_{s, s+1} q_{s+1, s}\right) x_{\mathbf{u}_{k s}} x_{\mathbf{u}_{1 j}}\right) \\
& =\gamma_{s t}^{k j} x_{\mathbf{u}_{k s}}\left(x_{\mathbf{u}_{1 j}} x_{\mathbf{u}_{1 t}}-\chi\left(\mathbf{u}_{1 j}, \mathbf{u}_{1 t}\right) x_{\mathbf{u}_{1 t}} x_{\mathbf{u}_{1 j}}\right) .
\end{aligned}
$$

To conclude, we prove the ninth case in a similar way:

$$
\begin{aligned}
{\left[x_{\mathbf{v}_{s t}}, x_{\mathbf{u}_{k j}}\right]_{c}=} & {\left[x_{\mathbf{v}_{s t}},\left[x_{\mathbf{u}_{k t}}, x_{\mathbf{u}_{t+1, j}}\right]_{c}\right]_{c} } \\
= & \gamma_{s t}^{k t}\left(1-q_{\mathbf{v}_{1 t}}\right)\left[x_{\mathbf{u}_{k s}} x_{\mathbf{u}_{1 t}}^{2}, x_{\mathbf{u}_{t+1, j}}\right] \\
& \quad+\chi\left(\mathbf{v}_{s t}, \mathbf{u}_{k, t}\right) x_{\mathbf{u}_{k t}} x_{\mathbf{v}_{s j}}-\chi\left(\mathbf{u}_{k t}, \mathbf{u}_{t+1, j}\right) x_{\mathbf{v}_{s j}} x_{\mathbf{u}_{k t}} .
\end{aligned}
$$

We consider the remaining commutator $\left[x_{\mathbf{v}_{s t}}, x_{\mathbf{u}_{j k}}\right]_{c}$ : when $j=1$.

Lemma 5.16. Let $s<t$ in $\{1, \ldots, \theta\}$. The following relations hold in $\mathfrak{B}$ :

$$
\begin{aligned}
& {\left[x_{\mathbf{v}_{s t}}, x_{\mathbf{u}_{1 k}}\right]_{c}=0 \quad \text { if } s<k \leq t,} \\
& {\left[x_{\mathbf{u}_{1 s}}, x_{\mathbf{v}_{s t}}\right]_{c}=0 .}
\end{aligned}
$$

Proof. By assumption we have

$$
\begin{aligned}
{\left[x_{\mathbf{v}_{12}}, x_{\mathbf{u}_{12}}\right]_{c} } & =\left[\left(\operatorname{ad}_{c} x_{1}\right)^{2} x_{2},\left(\operatorname{ad}_{c} x_{1}\right) x_{2}\right]_{c}=0, \\
{\left[x_{\mathbf{v}_{13}}, x_{\mathbf{u}_{12}}\right]_{c} } & =\left[\left(\operatorname{ad}_{c} x_{1}\right)^{2}\left(\operatorname{ad}_{c} x_{2}\right) x_{3},\left(\operatorname{ad}_{c} x_{1}\right) x_{2}\right]_{c}=0 .
\end{aligned}
$$


For $t \geq 4,\left[x_{\mathbf{u}_{4} t}, x_{\mathbf{u}_{12}}\right]_{c}=0$ by (5-14), and using (1-4),

$$
\left[x_{\mathbf{v}_{1 t}}, x_{\mathbf{u}_{12}}\right]_{c}=\left[\left[x_{\mathbf{v}_{13}}, x_{\mathbf{u}_{4 t}}\right]_{c}, x_{\mathbf{u}_{12}}\right]_{c}=0 .
$$

For each $k \leq t$ we have $\left[x_{\mathbf{u}_{1 t}}, x_{\mathbf{u}_{3 k}}\right]_{c}=\left[x_{1}, x_{\mathbf{u}_{3 k}}\right]_{c}=0$, so $\left[x_{\mathbf{v}_{1 t}}, x_{\mathbf{u}_{3 k}}\right]_{c}=0$. Using (1-4) and (5-15) we have

$$
\left[x_{\mathbf{v}_{1 t}}, x_{\mathbf{u}_{1 k}}\right]_{c}=\left[x_{\mathbf{v}_{1 t}},\left[x_{\mathbf{u}_{12}}, x_{\mathbf{u}_{3 k}}\right]_{c}\right]_{c}=0 .
$$

Now consider $2 \leq s \leq k$. Since $\left[x_{\mathbf{v}_{1 t}}, x_{\mathbf{u}_{1 k}}\right]_{c}=\left[x_{\mathbf{u}_{2 s}}, x_{\mathbf{u}_{1 k}}\right]_{c}=0$ by previous results and (5-16), we conclude from (1-5) and Lemma 5.15 that (5-21) is valid in the general case.

To prove (5-22), we have for $s=1, t=2$

$$
\left[x_{\mathbf{u}_{11}}, x_{\mathbf{v}_{12}}\right]_{c}=\left[x_{1}, x_{\mathbf{v}_{12}}\right]_{c}=\left(\operatorname{ad}_{c} x_{1}\right)^{3} x_{2}=0 .
$$

Using that $\left[x_{1}, x_{\mathbf{u}_{3 t}}\right]_{c}=0$ if $t \geq 3$ and (1-4), we deduce that

$$
\left[x_{\mathbf{u}_{11}}, x_{\mathbf{v}_{1 t}}\right]_{c}=\left[x_{1},\left[x_{\mathbf{v}_{12}}, x_{\mathbf{u}_{3 t}}\right]_{c}\right]_{c}=0 .
$$

If $1<s<t$ we have, by the previous case,

$$
\left[x_{\mathbf{u}_{1 s}}, x_{\mathbf{v}_{1 t}}\right]_{c}=-\chi\left(x_{\mathbf{u}_{1 s}}, x_{\mathbf{v}_{1 t}}\right)\left[x_{\mathbf{v}_{1 t}}, x_{\mathbf{u}_{1 s}}\right]_{c}=0 .
$$

By (5-18), $\left[x_{\mathbf{u}_{1 s}}, x_{\mathbf{u}_{2 s}}\right]_{c}=0$. Also, $\left[x_{\mathbf{v}_{1 t}}, x_{\mathbf{u}_{2 s}}\right]_{c}=\chi\left(\mathbf{u}_{1 t}, \mathbf{u}_{2 s}\right) x_{\mathbf{v}_{s t}}$, by Lemma 5.15. Equation (5-22) follows by (1-4) and the last three equalities.

Lemma 5.17. Let $s<k<t$. The following relations hold in $\mathfrak{B}$ :

$$
\begin{aligned}
& {\left[x_{\mathbf{v}_{s k}}, x_{\mathbf{u}_{1 t}}\right]_{c}=\chi\left(\mathbf{v}_{s k}, \mathbf{u}_{1 k}\right)\left(1-q_{k, k+1} q_{k+1, k}\right) x_{\mathbf{u}_{1 k}} x_{\mathbf{v}_{s t}},} \\
& {\left[x_{\mathbf{u}_{1 s}}, x_{\mathbf{v}_{k t}}\right]_{c}=\chi\left(\mathbf{u}_{1 s}, \mathbf{u}_{1 k}\right)\left(1+q_{\mathbf{u}_{1 k}}\right)\left(1-q_{k, k+1} q_{k+1, k}\right) x_{\mathbf{u}_{1 k}} x_{\mathbf{v}_{s t}} .}
\end{aligned}
$$

Proof. The proof follows by (1-4), the second case of Lemma 5.15 and (5-22):

$$
\begin{aligned}
{\left[x_{\mathbf{v}_{s k}}, x_{\mathbf{u}_{1 t}}\right]_{c} } & =\left[x_{\mathbf{v}_{s k}},\left[x_{\mathbf{u}_{1 k}}, x_{\mathbf{u}_{k+1, t}}\right]_{c}\right]_{c} \\
& =\chi\left(\mathbf{v}_{s k}, \mathbf{u}_{1 k}\right) x_{\mathbf{u}_{1 k}} x_{\mathbf{v}_{s t}}-\chi\left(\mathbf{u}_{1 k}, \mathbf{u}_{k+1, t}\right) x_{\mathbf{v}_{s t}} x_{\mathbf{u}_{1 k}} \\
& =\chi\left(\mathbf{v}_{s k}, \mathbf{u}_{1 k}\right)\left(1-\chi\left(\mathbf{u}_{1 k}, \mathbf{u}_{k+1, t}\right) \chi\left(\mathbf{u}_{k+1, t}, \mathbf{u}_{1 k}\right)\right) x_{\mathbf{u}_{1 k}} x_{\mathbf{v}_{s t}} \\
{\left[x_{\mathbf{u}_{1 s}}, x_{\mathbf{v}_{k t}}\right]_{c} } & =\left[x_{\mathbf{u}_{1 s}},\left[x_{\mathbf{u}_{1 k}}, x_{\mathbf{u}_{1 t}}\right]_{c}\right]_{c} \\
& =\left[x_{\mathbf{v}_{s k}}, x_{\mathbf{u}_{1 t}}\right]_{c}+\chi\left(\mathbf{u}_{1 s}, \mathbf{u}_{1 k}\right) x_{\mathbf{u}_{1 k}} x_{\mathbf{v}_{s t}}-\chi\left(\mathbf{u}_{1 k}, \mathbf{u}_{1 t}\right) x_{\mathbf{v}_{s t}} x_{\mathbf{u}_{1 k}} \\
& =\chi\left(\mathbf{u}_{1 s}, \mathbf{u}_{1 k}\right)\left(q_{\mathbf{u}_{1 k}}\left(1-q_{k, k+1} q_{k+1, k}\right)+1-q_{k, k+1} q_{k+1, k}\right) x_{\mathbf{u}_{1 k}} x_{\mathbf{v}_{s t}}
\end{aligned}
$$

We next deal with the expression of the commutator of two words of type $x_{\mathbf{v}_{s t}}$. 
Lemma 5.18. Let $s<t$ and $s \leq k<j$, with $k \neq s$ or $j \neq t$. The following relations hold in $\mathfrak{B}$ :

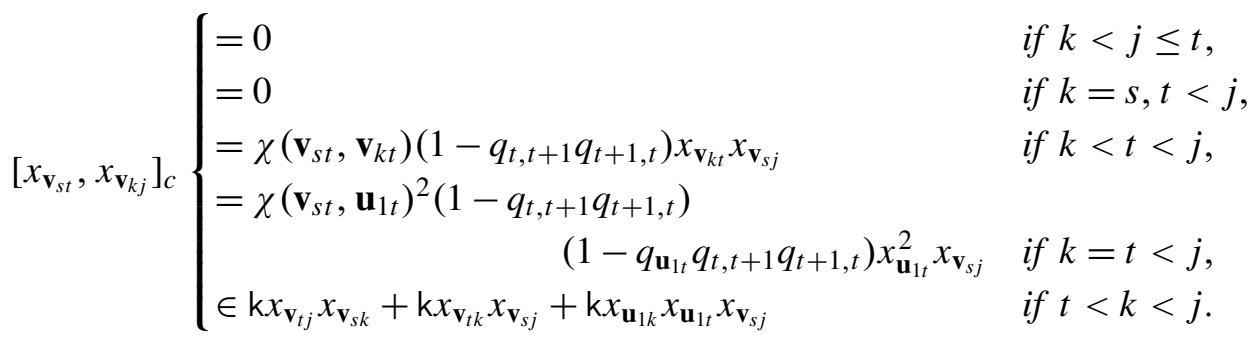

Proof. The first and second equalities follow from (1-4) and (5-21), (5-22), respectively. For the third, we use the previous one and (1-4):

$$
\begin{aligned}
& {\left[x_{\mathbf{v}_{s t}}, x_{\mathbf{v}_{k j}}\right]_{c}} \\
& =\left[x_{\mathbf{v}_{s t}},\left[x_{\mathbf{u}_{1 k}}, x_{\mathbf{u}_{1 j}}\right]_{c}\right]_{c} \\
& =\chi\left(\mathbf{v}_{s t}, \mathbf{u}_{1 k}\right) x_{\mathbf{u}_{1 k}}\left(\chi\left(\mathbf{v}_{s t}, \mathbf{u}_{1 t}\right)\left(1-q_{t, t+1} q_{t+1, t}\right) x_{\mathbf{u}_{1 t}} x_{\mathbf{v}_{s j}}\right) \\
& -\chi\left(\mathbf{u}_{1 k}, \mathbf{u}_{1 j}\right)\left(\chi\left(\mathbf{v}_{s t}, \mathbf{u}_{1 t}\right)\left(1-q_{t, t+1} q_{t+1, t}\right) x_{\mathbf{u}_{1 t}} x_{\mathbf{v}_{s j}}\right) x_{\mathbf{u}_{1 k}} \\
& =\left(1-q_{t, t+1} q_{t+1, t}\right)\left(\chi\left(\mathbf{v}_{s t}, \mathbf{u}_{1 k}\right) \chi\left(\mathbf{v}_{s t}, \mathbf{u}_{1 t}\right) x_{\mathbf{u}_{1 k}} x_{\mathbf{u}_{1 t}} x_{\mathbf{v}_{s j}}\right. \\
& \left.-\chi\left(\mathbf{u}_{1 k}, \mathbf{u}_{1 j}\right) \chi\left(\mathbf{v}_{s t}, \mathbf{u}_{1 t}\right) \chi\left(\mathbf{v}_{s j}, \mathbf{u}_{1 k}\right) x_{\mathbf{u}_{1 t}} x_{\mathbf{u}_{1 k}} x_{\mathbf{v}_{s j}}\right) \\
& =\chi\left(\mathbf{v}_{s t}, \mathbf{u}_{1 k}\right) \chi\left(\mathbf{v}_{s t}, \mathbf{u}_{1 t}\right)\left(1-q_{t, t+1} q_{t+1, t}\right)\left(x_{\mathbf{u}_{1 k}} x_{\mathbf{u}_{1 k}}-\chi\left(\mathbf{u}_{1 k}, \mathbf{u}_{1 t}\right) x_{\mathbf{u}_{1 k}} x_{\mathbf{u}_{1 k}}\right) x_{\mathbf{v}_{s j}} \text {. }
\end{aligned}
$$

The fourth case is similar to the previous one.

To prove the last case we use (1-4) and Lemma 5.17:

$$
\begin{aligned}
& {\left[x_{\mathbf{v}_{s t}}, x_{\mathbf{v}_{k j}}\right]_{c}=\left[x_{\mathbf{v}_{s t}},\left[x_{\mathbf{u}_{1 k}}, x_{\mathbf{u}_{1 j}}\right]_{c}\right]_{c}} \\
& =\left[\chi\left(\mathbf{v}_{s t}, \mathbf{u}_{1 t}\right)\left(1-q_{t, t+1} q_{t+1, t}\right) x_{\mathbf{u}_{1 t}} x_{\mathbf{v}_{s k}}, x_{\mathbf{u}_{1 j}}\right]_{c} \\
& +\chi\left(\mathbf{v}_{s t}, \mathbf{u}_{1 k}\right) x_{\mathbf{u}_{1 k}}\left(\chi\left(\mathbf{v}_{s t}, \mathbf{u}_{1 t}\right)\left(1-q_{t, t+1} q_{t+1, t}\right) x_{\mathbf{u}_{1 t}} x_{\mathbf{v}_{s j}}\right) \\
& -\chi\left(\mathbf{u}_{1 k}, \mathbf{u}_{1 j}\right)\left(\chi\left(\mathbf{v}_{s t}, \mathbf{u}_{1 t}\right)\left(1-q_{t, t+1} q_{t+1, t}\right) x_{\mathbf{u}_{1 t}} x_{\mathbf{v}_{s j}}\right) x_{\mathbf{u}_{1 k}} .
\end{aligned}
$$

The proof is finished using (1-5) and the previous identities.

Theorem 5.19. Let $V$ be a standard braided vector space of type $B_{\theta}$, where $\theta=$ $\operatorname{dim} V$, and let $C=\left(a_{i j}\right)_{i, j \in\{1, \ldots, \theta\}}$ be the corresponding Cartan matrix of type $B_{\theta}$.

The Nichols algebra $\mathfrak{B}(V)$ is presented by the generators $x_{i}, 1 \leq i \leq \theta$, and the relations

$$
\begin{aligned}
x_{\alpha}^{N_{\alpha}} & =0, & & \alpha \in \Delta^{+} ; \\
\operatorname{ad}_{c}\left(x_{i}\right)^{1-a_{i j}}\left(x_{j}\right) & =0, & & i \neq j ; \\
{\left[\left(\operatorname{ad} x_{j-1}\right)\left(\operatorname{ad} x_{j}\right) x_{j+1}, x_{j}\right]_{c} } & =0, & & 1<j<\theta, q_{j j}=-1 ; \\
{\left[\left(\operatorname{ad} x_{1}\right)^{2} x_{2},\left(\operatorname{ad} x_{1}\right) x_{2}\right]_{c} } & =0, & & q_{11} \in \mathbb{G}_{3} \text { or } q_{22}=-1 ; \\
{\left[\left(\operatorname{ad} x_{1}\right)^{2}\left(\operatorname{ad} x_{2}\right) x_{3},\left(\operatorname{ad} x_{1}\right) x_{2}\right]_{c} } & =0, & & q_{11} \in \mathbb{G}_{3} \text { or } q_{22}=-1 .
\end{aligned}
$$


The following elements constitute a basis of $\mathfrak{B}(V)$ :

$$
x_{\beta_{1}}^{h_{1}} x_{\beta_{2}}^{h_{2}} \ldots x_{\beta_{P}}^{h_{P}} \text {, where } 0 \leq h_{j}<N_{\beta_{j}}-1 \text { if } \beta_{j} \in S_{I} \text {, for } 1 \leq j \leq P \text {. }
$$

Proof. The proof is analogous to that of Theorem 5.14, since by the previous lemmas we can express the commutator of two generators $x_{\alpha}<x_{\beta}$ as a linear combination of monotone hyperwords whose greater hyperletter is great or equal to $x_{\beta}$.

5D. Presentation when the type is $\boldsymbol{G}_{2}$. We now consider standard braidings of type $G_{2}$, with $m_{12}=3, m_{21}=1$.

Lemma 5.20. Let $\mathfrak{B}:=T(V) / I$, for some $I \in \mathfrak{S}$, and suppose that

$$
x_{1}^{\text {ord } q_{11}}=0, \quad x_{2}^{\text {ord } q_{22}}=0, \quad\left(\operatorname{ad} x_{1}\right)^{4} x_{2}=\left(\operatorname{ad} x_{2}\right)^{2} x_{1}=0
$$

in $\mathfrak{B}$. Then

(a) $\left[x_{1}^{3} x_{2} x_{1} x_{2}\right]_{c}=0$ in $\mathfrak{B} \Longleftrightarrow 4 e_{1}+2 e_{2} \notin \Delta^{+}(\mathfrak{B})$.

Assume further that the equivalent conditions in (a) hold. Then

(b) $\left[\left(\operatorname{ad} x_{1}\right)^{3} x_{2},\left(\operatorname{ad} x_{1}\right)^{2} x_{2}\right]_{c}=0$ in $\mathfrak{B} \Longleftrightarrow 5 e_{1}+2 e_{2} \notin \Delta^{+}(\mathfrak{B})$ and

(c) $\left[\left[x_{1}^{2} x_{2} x_{1} x_{2}\right]_{c},\left[x_{1} x_{2}\right]_{c}\right]_{c}=0$ in $\mathfrak{B} \Longleftrightarrow 4 e_{1}+3 e_{2} \notin \Delta^{+}(\mathfrak{B})$.

Assume also that the equivalent conditions in (b) and those in (c) hold. Then

(d) $\left[\left[x_{1}^{2} x_{2}\right]_{c},\left[x_{1}^{2} x_{2} x_{1} x_{2}\right]_{c}\right]_{c}=0$ in $\mathfrak{B} \Longleftrightarrow 5 e_{1}+3 e_{2} \notin \Delta^{+}(\mathfrak{B})$.

In particular, all these relations hold when $V$ is a standard braiding and $\mathfrak{B}=$ $\mathfrak{B}(V)$ is finite-dimensional.

Proof. Take the ordering $x_{1}<x_{2}$, and consider a PBW basis as in Theorem 1.12. Define $\gamma_{k}:=\prod_{0 \leq j \leq k-1}\left(1-q_{11}^{j} q_{12} q_{21}\right)$.

(a) If $\left[x_{1}^{3} x_{2} x_{1} x_{2}\right]_{c}=0$, then $4 e_{1}+2 e_{2} \notin \Delta^{+}(\mathfrak{B})$ since there are no possible Lyndon words in $S_{I}: x_{1}^{3} x_{2} x_{1} x_{2}$ is the unique Lyndon word such that $x_{1}^{3} x_{2}$ and $x_{1} x_{2}^{2}$ are not factors, and it is not in $S_{I}$ by assumption.

Conversely, if $4 e_{1}+2 e_{2} \notin \Delta^{+}(\mathfrak{B})$, then $\left[x_{1}^{3} x_{2} x_{1} x_{2}\right]_{c}$ is a linear combination of greater hyperwords, and $\left[x_{1} x_{2} x_{1}^{3} x_{2}\right]_{c}$ and $\left[x_{1}^{2} x_{1}^{2} x_{2}\right]$ are the only greater hyperwords that are not in $S_{I}$ and do not end in $x_{1}$ (we discard words ending in $x_{1}$ since $\left[x_{1}^{3} x_{2} x_{1} x_{2}\right]_{c}$ is in ker $D_{1}$ ). Taking their Shirshov decomposition, we see that there exist $\alpha, \beta \in \mathrm{k}$ such that

$$
\left[x_{1}^{3} x_{2} x_{1} x_{2}\right]_{c}-\alpha\left[x_{1} x_{2}\right]_{c}\left[x_{1}^{3} x_{2}\right]_{c}-\beta\left[x_{1}^{2} x_{2}\right]_{c}^{2}=0 .
$$

Note that $\left[x_{1}^{3} x_{2} x_{1} x_{2}\right]_{c}=\operatorname{ad} x_{1}\left(\left[x_{1}^{2} x_{2} x_{1} x_{2}\right]_{c}\right)$, so by direct calculation,

$$
D_{2}\left(\left[x_{1}^{2} x_{2} x_{1} x_{2}\right]_{c}\right)=0 .
$$


Apply $D_{2}$ to both sides of equality (5-27) and express the result as a linear combination of $\left[x_{1}^{3} x_{2}\right]_{c} x_{1},\left[x_{1}^{2} x_{2}\right]_{c} x_{1}^{2}$ and $\left[x_{1} x_{2}\right]_{c} x_{1}^{3}$. The coefficient of $\left[x_{1} x_{2}\right]_{c} x_{1}^{3}$ is

$$
\alpha\left(1-q_{12} q_{21}\right)\left(1-q_{11} q_{12} q_{21}\right),
$$

so $\alpha=0$. Note also that $D_{1}^{2} D_{2}\left(\left[x_{-} 1^{3} x_{2} x_{1} x_{2}\right]_{c}\right)=0$; but

$$
D_{1}^{2} D_{2}\left(\left[x_{1}^{2} x_{2}\right]_{c}^{2}\right)=\left(1-q_{12} q_{21}\right)\left(1-q_{11} q_{12} q_{21}\right)\left(1+q_{11}\right)\left(q_{2 e_{1}+e_{2}}+1\right)\left[x_{1}^{2} x_{2}\right]_{c} .
$$

Looking at the proof of Proposition 4.7, we see that $q_{2 e_{1}+e_{2}} \neq-1$, so $\beta=0$.

(b) Assuming (5-26) and the condition in (a), the only possible Lyndon word of degree $5 e_{1}+2 e_{j}$ is $x_{1}^{3} x_{2} x_{1}^{2} x_{2}$, and

$$
\left[x_{1}^{2} x_{2} x_{1} x_{2} x_{1} x_{2}\right]_{c}=\left[\left(\operatorname{ad} x_{1}\right)^{3} x_{2},\left(\operatorname{ad} x_{1}\right)^{2} x_{2}\right]_{c} .
$$

Then we proceed as before. One implication is clear. For the other, if $5 e_{1}+2 e_{j} \notin$ $\Delta^{+}(\mathfrak{B})$, there exists $\alpha \in \mathrm{k}$ such that

$$
\left[\left(\operatorname{ad} x_{1}\right)^{3} x_{2},\left(\operatorname{ad} x_{1}\right)^{2} x_{2}\right]_{c}=\alpha\left(\operatorname{ad} x_{1}\right)^{2} x_{2}\left(\operatorname{ad} x_{1}\right)^{3} x_{2} .
$$

Now we apply $D_{2}$ and express the equality as a linear combination of $\left(\operatorname{ad} x_{1}\right)^{3} x_{2} x_{1}^{2}$ and $\left(\operatorname{ad} x_{1}\right)^{2} x_{2} x_{1}^{3}$ (using the hypothesis that $\left.\left(\operatorname{ad} x_{1}\right)^{4} x_{2}=0\right)$; the coefficient of $\left(\operatorname{ad} x_{1}\right)^{2} x_{2} x_{1}^{3}$ is $\alpha \gamma_{3}$, so $\alpha=0$.

(c) The proof is similar. Since we are considering Lyndon words not having $x_{1}^{3} x_{2}$ or $x_{1} x_{2}^{2}$ as a factor, the only possible Lyndon word of degree $4 e_{1}+3 e_{j}$ is $x_{1}^{2} x_{2} x_{1} x_{2} x_{1} x_{2}$, and

$$
\left[x_{1}^{2} x_{2} x_{1} x_{2} x_{1} x_{2}\right]_{c}=\left[\left[x_{1}^{2} x_{2} x_{1} x_{2}\right]_{c},\left[x_{1} x_{2}\right]_{c}\right]_{c} .
$$

If $4 e_{1}+3 e_{j} \notin \Delta^{+}(\mathfrak{B})$, there exist $\alpha_{i} \in \mathrm{k}$ such that

$$
\begin{aligned}
& {\left[x_{1}^{2} x_{2}\left(x_{1} x_{2}\right)^{2}\right]_{c}} \\
& \quad=\alpha_{1}\left[x_{1} x_{2}\right]_{c}\left[x_{1}^{2} x_{2} x_{1} x_{2}\right]_{c}+\alpha_{2}\left[x_{1} x_{2}\right]_{c}^{2}\left[x_{1}^{2} x_{2}\right]_{c}+\alpha_{3} x_{2}\left[x_{1}^{2} x_{2}\right]_{c}^{2}+\alpha_{4} x_{2}\left[x_{1} x_{2}\right]_{c}\left[x_{1}^{3} x_{2}\right]_{c},
\end{aligned}
$$

since, as above, we are discarding words greater than $x_{1}^{2} x_{2} x_{1} x_{2} x_{1} x_{2}$ ending in $x_{1}$; we also discard words with factors $x_{1}^{4} x_{2}, x_{1} x_{2}^{2}, x_{1}^{3} x_{2} x_{1}^{2} x_{2}$, by the assumption on $\mathfrak{B}$. We apply $D_{2}$ to this equality. Using the definition of the braided commutator, we express the hyperletter just considered as a linear combination of elements of the PBW basis, having degree $4 e_{1}+2 e_{2}$.

The coefficient of $x_{2}\left[x_{1} x_{2}\right]_{c} x_{1}^{3}$ is $\alpha_{4} \gamma_{3}$ since this PBW generator appears only in the expression of $D_{2}\left(x_{2}\left[x_{1} x_{2}\right]_{c}\left[x_{1}^{3} x_{2}\right]_{c}\right)$. Thus $\alpha_{4}=0$.

Using this fact, we see that the coefficient of $x_{2}\left[x_{1}^{3} x_{2}\right]_{c} x_{1}$ is

$$
\alpha_{3} \gamma_{2}\left(1+q_{11}\right) q_{11}^{2} q_{12} q_{21}^{2} q_{22}
$$

since this term appears only in the expression of $D_{j}\left(x_{2}\left[x_{1}^{2} x_{2}\right]_{c}^{2}\right)$. Thus $\alpha_{3}=0$. 
Next, the coefficient of $\left[x_{1} x_{2}\right]_{c}^{2} x_{1}^{2}$ is $\alpha_{2} \gamma_{2}$, so $\alpha_{2}=0$. Now we calculate the coefficient of $\left[x_{1}^{2} x_{2}\right]_{c}^{2}$ :

$$
\alpha_{1} \gamma_{2}\left(\chi\left(\mathbf{e}_{1}, 5 \mathbf{e}_{1}+\mathbf{e}_{2}\right)-\chi\left(2 \mathbf{e}_{1}+\mathbf{e}_{2}, \mathbf{e}_{1}+\mathbf{e}_{2}\right)\right)=\alpha_{1} \gamma_{2} q_{11} q_{12}\left(q_{11}^{3}-q_{22} q_{12} q_{21}\right) .
$$

Since $q_{11}^{3} \neq q_{22} q_{12} q_{21}$ for each standard braiding, we conclude that $\alpha_{1}=0$.

(d) If the conditions in (b) and (c) hold, the only possible Lyndon word of degree $5 e_{1}+3 e_{2}$ not having $x_{1}^{4} x_{2}$ or $x_{1} x_{2}^{2}$ as factors is $x_{1}^{2} x_{2} x_{1}^{2} x_{2} x_{1} x_{2}$, and

$$
\left[x_{1}^{2} x_{2} x_{1}^{2} x_{2} x_{1} x_{2}\right]_{c}=\left[\left[x_{1}^{2} x_{2}\right]_{c},\left[x_{1}^{2} x_{2} x_{1} x_{2}\right]_{c}\right]_{c} .
$$

This hyperword is not in $S_{I}$ if and only if there exist $v_{i} \in \mathrm{k}$ such that

$$
\begin{aligned}
{\left[x_{1}^{2} x_{2} x_{1}^{2} x_{2} x_{1} x_{2}\right]_{c}=v_{1}\left[x_{1}^{2} x_{2} x_{1} x_{2}\right]_{c}[} & \left.x_{1}^{2} x_{2}\right]_{c}+v_{2}\left[x_{1} x_{2}\right]_{c}\left[x_{1}^{2} x_{2}\right]_{c}^{2} \\
& +v_{3}\left[x_{1} x_{2}\right]_{c}^{2}\left[x_{1}^{3} x_{2}\right]_{c}+v_{4} x_{2}\left[x_{1}^{2} x_{2}\right]_{c}\left[x_{1}^{3} x_{2}\right]_{c} .
\end{aligned}
$$

Apply $D_{2}$ and note that $D_{2}\left(\left[x_{1}^{2} x_{2} x_{1}^{2} x_{2} x_{1} x_{2}\right]_{c}\right)=0$ under the hypotheses on $\mathfrak{B}$. Then express the resulting sum as a linear combination of elements of the PBW basis, which have degree $5 e_{1}+2 e_{2}$.

The hyperword $x_{2}\left[x_{1}^{2} x_{2}\right] x_{1}^{3}$ appears only for $D_{2}\left(x_{2}\left[x_{1}^{2} x_{2}\right]_{c}\left[x_{1}^{3} x_{2}\right]_{c}\right)$, and its coefficient is $v_{4} \gamma_{3}$, and since $\gamma_{3} \neq 0$ we conclude that $\nu_{4}=0$.

Analogously, $\left[x_{1} x_{2}\right]_{c}^{2} x_{1}^{3}$ appears only for $\left[x_{1} x_{2}\right]_{c}^{2}\left[x_{1}^{3} x_{2}\right]_{c}$ (due to $v_{4}=0$ ). Its coefficient is $\nu_{3} \gamma_{3}$, so $v_{3}=0$.

Note that $D_{1}^{2} D_{2}\left(\left[x_{1}^{2} x_{2} x_{1} x_{2}\right]_{c}\right)=0$. We apply $D_{1}^{2} D_{2}$ to the expression (5-28), and obtain

$$
0=v_{1} \gamma_{2}\left(1+q_{11}\right)\left[x_{1}^{2} x_{2} x_{1} x_{2}\right]_{c}+v_{2} \gamma_{2}\left(1+q_{11}\right)\left(1+q_{2 \mathbf{e}_{1}+\mathbf{e}_{2}}\right)\left[x_{1} x_{2}\right]_{c}\left[x_{1}^{2} x_{2}\right]_{c} .
$$

The terms $\left[x_{1}^{2} x_{2} x_{1} x_{2}\right]_{c}$ and $\left[x_{1} x_{2}\right]_{c}\left[x_{1}^{2} x_{2}\right]_{c}$ are linearly independent, since they are linearly independent in $\mathfrak{B}(V)$, and we have a surjection $\mathfrak{B} \rightarrow \mathfrak{B}(V)$. Then

$$
v_{1} \gamma_{2}\left(1+q_{11}\right)=v_{2} \gamma_{2}\left(1+q_{11}\right)\left(1+q_{2 \mathbf{e}_{1}+\mathbf{e}_{2}}\right)=0 .
$$

But for standard braidings of type $G_{2}$ we note that $q_{11}, q_{2 \mathbf{e}_{1}+\mathbf{e}_{2}} \neq-1$ and $\gamma_{2} \neq 0$, so $v_{1}=v_{2}=0$.

The last statement is true since

$$
\Delta^{+}(\mathfrak{B}(V))=\left\{e_{1}, e_{1}+e_{2}, 2 e_{1}+e_{2}, 3 e_{1}+e_{2}, 3 e_{1}+2 e_{2}, e_{2}\right\},
$$

if the braiding is standard of type $G_{2}$.

Remark 5.21. Let $V$ be a standard braided vector space of type $G_{2}$ and let $\mathfrak{B}$ be a braided graded Hopf algebra satisfying the hypotheses of Lemma 5.20. In a similar way to Lemma 5.5, if $q_{11} \notin \mathbb{G}_{4}$ and $q_{22} \neq-1$, then $5 e_{1}+2 e_{2}, 4 e_{1}+2 e_{2} 4 e_{1}+$ $3 e_{2}, 5 e_{1}+3 e_{2} \notin \Delta^{+}(\mathfrak{B})$. 
This follows because $x_{1}^{3} x_{2} x_{1}^{2} x_{2}, x_{1}^{2} x_{2} x_{1} x_{2} x_{1} x_{2}, x_{1}^{2} x_{2} x_{1}^{2} x_{2} x_{1} x_{2} \notin S_{I}$, using the quantum Serre relations as in the lemma cited.

Theorem 5.22. Let $V$ be a standard braided vector space of type $G_{2}$. The Nichols algebra $\mathfrak{B}(V)$ is presented by the generators $x_{1}, x_{2}$ and the relations

$$
\operatorname{ad}_{c}\left(x_{1}\right)^{4}\left(x_{2}\right)=\operatorname{ad}_{c}\left(x_{2}\right)^{2}\left(x_{1}\right)=0, \quad x_{\alpha}^{N_{\alpha}}=0, \alpha \in \Delta^{+},
$$

and, if $q_{11} \in \mathbb{G}_{4}$ or $q_{22}=-1$,

$$
\begin{aligned}
{\left[\left(\operatorname{ad} x_{1}\right)^{3} x_{2},\left(\operatorname{ad} x_{1}\right)^{2} x_{2}\right]_{c} } & =0, \\
{\left[x_{1},\left[x_{1}^{2} x_{2} x_{1} x_{2}\right]_{c}\right]_{c} } & =0, \\
{\left[\left[x_{1}^{2} x_{2} x_{1} x_{2}\right]_{c},\left[x_{1} x_{2}\right]_{c}\right]_{c} } & =0, \\
{\left[\left[x_{1}^{2} x_{2}\right]_{c},\left[x_{1}^{2} x_{2} x_{1} x_{2}\right]_{c}\right]_{c} } & =0 .
\end{aligned}
$$

The following elements constitute a basis of $\mathfrak{B}(V)$ :

$x_{2}^{h_{e_{2}}}\left[x_{1} x_{2}\right]_{c}^{h_{e_{1}+e_{2}}^{h_{2}}}\left[x_{1}^{2} x_{2} x_{1} x_{2}\right]_{c}^{h_{3 e_{1}+2 e_{2}}}\left[x_{1}^{2} x_{2}\right]_{c}^{h_{2 e_{1}+e_{2}}}\left[x_{1}^{3} x_{2}\right]_{c}^{h_{3 e_{1}+e_{2}}} x_{1}^{h_{e_{1}}}, \quad 0 \leq h_{\alpha} \leq N_{\alpha}-1$.

Proof. The statement about the PBW basis follows from Corollary 4.2 and the definitions of the $x_{\alpha}$.

Let $\mathfrak{B}$ be the algebra presented by the generators $x_{1}, x_{2}$ and the relations (5-29)(5-33). From Lemma 5.20 and Corollary 5.2, we have a canonical epimorphism of algebras $\phi: \mathfrak{B} \rightarrow \mathfrak{B}(V)$.

Consider the subspace $\mathscr{I}$ of $\mathfrak{B}$ generated by the elements in (5-34). We prove by induction on the sum $S$ of the $h_{\alpha}$ 's of a such product $M$ that $x_{1} M \in \mathscr{I}$; moreover, we prove that it is a linear combination of products whose first hyperletter is less than or equal to the first hyperletter of $M$. If $S=0$, we have $M=1$.

- If $M=x_{1}^{N_{1}}$, then $x_{1} M=x_{1}^{N_{1}+1}$, which is zero if $N_{1}=$ ord $x_{1}-1$.

- If $M=\left[x_{1}^{3} x_{2}\right]_{c} M^{\prime}$, then we use that $x_{1}\left[x_{1}^{3} x_{2}\right]_{c}=q_{11}^{3} q_{12}\left[x_{1}^{3} x_{2}\right]_{c} x_{1}$ to prove that $x_{1} M$ lies in $\mathscr{I}$ and either is zero or begins with $\left[x_{1}^{3} x_{2}\right]_{c}$.

- If $M=\left[x_{1}^{2} x_{2}\right]_{c} M^{\prime}$, we have

$$
x_{1}\left[x_{1}^{2} x_{2}\right]_{c}=\left[x_{1}^{3} x_{2}\right]_{c}+q_{11}^{2} q_{12}\left[x_{1}^{2} x_{2}\right]_{c} x_{1} .
$$

We use the inductive step and relation (5-30) to prove that $x_{1} M$ lies in $\mathscr{I}$ and is either zero or a linear combination of hyperwords starting with a hyperletter less than or equal to $\left[x_{1}^{2} x_{2}\right]_{c}$.

- If $M=\left[x_{1}^{2} x_{2} x_{1} x_{2}\right]_{c} M^{\prime}$, we deduce from (5-31) that

$$
x_{1}\left[x_{1}^{2} x_{2} x_{1} x_{2}\right]_{c}=\chi\left(\mathbf{e}_{1}, 3 \mathbf{e}_{1}+2 \mathbf{e}_{2}\right)\left[x_{1}^{2} x_{2} x_{1} x_{2}\right]_{c} x_{1}
$$


using also (5-32) and (5-33), we prove that $x_{1} M$ lies in $\Phi$ and is either zero or a linear combination of hyperwords that starting with a hyperletter less than or equal to $\left[x_{1}^{2} x_{2} x_{1} x_{2}\right]_{c}$.

- If $M=\left[x_{1} x_{2}\right]_{c} M^{\prime}$, observe that

$$
x_{1}\left[x_{1} x_{2}\right]_{c}=\left[x_{1}^{2} x_{2}\right]_{c}+q_{11} q_{12}\left[x_{1} x_{2}\right]_{c} x_{1} .
$$

Using the inductive step together with (5-31), (5-32), and the equality

$$
\left[x_{1}^{2} x_{2}\right]_{c}\left[x_{1} x_{2}\right]_{c}=\left[\left[x_{1}^{2} x_{2}\right]_{c},\left[x_{1} x_{2}\right]_{c}\right]_{c}+\chi\left(2 \mathbf{e}_{1}+\mathbf{e}_{2}, \mathbf{e}_{1}+\mathbf{e}_{2}\right)\left[x_{1} x_{2}\right]_{c}\left[x_{1}^{2} x_{2}\right]_{c},
$$

by the definition of braided commutator, we prove that $x_{1} M$ lies in $\mathscr{I}$ and is either zero or a linear combination of hyperwords starting with a hyperletter less than or equal to $\left[x_{1} x_{2}\right]_{c}$.

- If $M=x_{2} M^{\prime}$, we use the equalities $x_{1} x_{2}=\left[x_{1} x_{2}\right]_{c}+q_{12} x_{2} x_{1}$ and $\left[\left[x_{1} x_{2}\right]_{c}, x_{2}\right]_{c}=$ 0 to prove that $x_{1} M$ lies in $\mathscr{I}$ and is either zero or a linear combination of hyperwords.

Now, $x_{2} M$ is a product of nonincreasing hyperwords or is zero, for each element in (5-34), so $\mathscr{I}$ is an ideal of $\mathfrak{B}$ containing 1 ; hence $\mathscr{I}=\mathfrak{B}$. Since the elements in (5-34) are a basis of $\mathfrak{B}(V)$, the map $\phi$ is an isomorphism.

5E. Presentation when the braiding is of Cartan type. In this subsection, we present the Nichols algebra of a diagonal braiding vector space of Cartan type with matrix $\left(q_{i j}\right)$, by generators and relations. This was established in [Andruskiewitsch and Schneider 2002a, Theorem 4.5] assuming that $q_{i i}$ has odd order and that order is not divisible by 3 if $i$ belongs to a component of type $G_{2}$. The proof in loc. cit. combines a reduction to symmetric $\left(q_{i j}\right)$ by twisting, with results from [Andersen et al. 1994] and [De Concini and Procesi 1993]. We also note that some particular instances were already proved earlier in this section.

Fix a standard braided vector space $V$ with connected Dynkin diagram and an $i \in\{1, \ldots, \theta\}$. Suppose that $\mathfrak{B}$ is a quotient by an ideal $I \in \mathfrak{S}$ of $T(V)$. Assume moreover that $V$ is not of type $G_{2}$ and that

(5-3) holds in $\mathfrak{B}$ if $1 \leq i \neq j \leq \theta$;

(5-4) holds in $\mathfrak{B}$ if $m_{k j}=m_{k l}=1$ and $m_{j l}=0$;

(5-6) holds in $\mathfrak{B}$ if $m_{k j}=2$ and $m_{j k}=1$;

(5-8) holds in $\mathfrak{B}$ if $m_{k j}=2, m_{j k}=m_{j l}=1$ and $m_{k l}=0$.

Note that if (5-3) holds in an algebra with derivations $D_{k}$, then (2-11) holds also, by Lemma 2.7. By Theorem 2.6, we have an algebra $s_{i}(\mathfrak{B})$ provided with skew derivations $D_{i}$. We set $\tilde{x}_{k}=\left(\operatorname{ad}_{c} x_{i}\right)^{m_{i k}}\left(x_{k}\right) \# 1 \in s_{i}(\mathfrak{B})$, for $k \neq i$, and $\tilde{x}_{i}=1 \# y$. The elements generate $s_{i}(\mathfrak{B})^{1}$ as a vector space. 
Lemma 5.23. Conditions (5-35)-(5-38) are satisfied with $s_{i}(\mathfrak{B})$ in lieu of $\mathfrak{B}$.

Proof of (5-35). Each $m \mathbf{e}_{k}+e_{j}, 0 \leq m \leq m_{k j}$ is an element of $\Delta\left(\mathfrak{B}\left(V_{i}\right)\right)$, so $s_{i}\left(m \mathbf{e}_{k}+\mathbf{e}_{j}\right) \in \Delta(\mathfrak{B}(V))$. Since we have a surjective morphism of braided graded Hopf algebras $\mathfrak{B} \rightarrow \mathfrak{B}(V)$, we have $\Delta(\mathfrak{B}(V)) \subseteq \Delta(\mathfrak{B})$.

From Lemma 5.3, $\left(\operatorname{ad}_{c} \tilde{x}_{k}\right)^{m} \tilde{x}_{j}=0$ if and only if $\tilde{x}_{k}^{m} \tilde{x}_{j}$ is a linear combination of greater words, for an order in which $\tilde{x}_{k}<\tilde{x}_{j}$ (since we are considering the Cartan case, the condition about the ordering of the $\tilde{x}_{j}$ is satisfied). Note that $\tilde{x}_{k}^{m} \tilde{x}_{j}$ is the unique Lyndon word of degree $m \mathbf{e}_{k}+e_{j}$. Then, by the relation (2-15) between the Hilbert series of $\mathfrak{B}$ and $s_{i}(\mathfrak{B})$, the validity of (5-3) for $s_{i}(\mathfrak{B})$ is equivalent to the condition

$$
s_{i}\left(\left(m_{k j}+1\right) \mathbf{e}_{k}+\mathbf{e}_{j}\right) \notin \Delta^{+}(\mathfrak{B}) .
$$

(a) When $k=i \neq j$, this says that $-\mathbf{e}_{i}+\mathbf{e}_{j} \notin \Delta^{+}(\mathfrak{B})$, so (5-3) holds.

(b) To prove (5-3) for $s_{i}(\mathfrak{B})$ when $j=i$, we show case by case that

$$
\left(m_{k i}+1\right) \mathbf{e}_{k}+\left(\left(m_{k i}+1\right) m_{i k}-1\right) \mathbf{e}_{i} \notin \Delta^{+}(\mathfrak{B}) .
$$

- If $m_{k i}=m_{i k}=0$, we have $\mathbf{e}_{k}-\mathbf{e}_{i} \notin \Delta^{+}(\mathfrak{B})$.

- If $m_{k i}=m_{i k}=1$, then $2 \mathbf{e}_{k}+\mathbf{e}_{i} \notin \Delta^{+}(\mathfrak{B})$, because $\left(\operatorname{ad} x_{k}\right)^{2} x_{i}=0$.

- If $m_{k i}=1$ and $m_{i k}=2$, then $2 \mathbf{e}_{k}+3 \mathbf{e}_{i} \notin \Delta^{+}(\mathfrak{B})$, since we can apply Lemma 5.5 to $\mathfrak{B}$, which satisfies (5-6) by assumption.

- If $m_{k i}=2$ and $m_{i k}=1$, then $3 \mathbf{e}_{k}+2 \mathbf{e}_{i} \notin \Delta^{+}(\mathfrak{B})$, as before.

Thus (5-3) holds for each $k \neq i$.

(c) Now consider $\theta \geq 3$ and $k, j \neq i$.

- If $m_{i k}=m_{i j}=0$, then $s_{i}\left(m \mathbf{e}_{k}+\mathbf{e}_{j}\right)=m \mathbf{e}_{k}+\mathbf{e}_{j}$, and $\left(m_{k j}+1\right) \mathbf{e}_{k}+\mathbf{e}_{j} \notin \Delta^{+}(\mathfrak{B})$, since the quantum Serre relation holds in $\mathfrak{B}$.

- If $m_{i k}=1$ and $m_{i j}=0$, then $s_{i}\left(m \mathbf{e}_{k}+\mathbf{e}_{j}\right)=m \mathbf{e}_{i}+m \mathbf{e}_{k}+\mathbf{e}_{j}$. If we assume $x_{j}<x_{i}<x_{k}$ and look at the possible Lyndon words in $S_{I}$, from (5-3), these words have no factors $x_{i}^{2} x_{k}, x_{j} x_{i}$, so the only possibility is $x_{j}\left(x_{k} x_{i}\right)^{m}$.

- If $m_{k j}=0$, then $x_{j} x_{k} x_{i}=q_{j k} x_{k} x_{j} x_{i}$, so $x_{j} x_{k} x_{i} \notin S_{I}$.

- If $m_{k j}=1$, then $x_{j} x_{k} x_{l} x_{k} \notin S_{I}$ when $m_{k i}=1$, since (5-4) is valid in $\mathfrak{B}$; while if $m_{k i}=2$ we have $q_{k k} \neq-1$ and

$$
\begin{aligned}
x_{j}\left(x_{k} x_{i}\right)^{2}= & \left(1+q_{k k}\right)^{-1} q_{k i}^{-1} x_{j} x_{k}^{2} x_{i}^{2}+\left(1+q_{k k}\right)^{-1} q_{k i} q_{k k}^{2} x_{j} x_{i} x_{k}^{2} x_{i} \\
= & q_{k i}^{-1} q_{k j}^{-1} q_{k k}^{-2} x_{k} x_{j} x_{k} x_{i}^{2}+\left(1+q_{k k}\right)^{-1} q_{k i}^{-1} q_{k j}^{-2} q_{k k}^{-2} x_{k}^{2} x_{j} x_{i}^{2} \\
& +\left(1+q_{k k}\right)^{-1} q_{k i} q_{k k}^{2} q_{j i} x_{i} x_{j} x_{k}^{2} x_{i} .
\end{aligned}
$$

In both cases, $x_{j}\left(x_{k} x_{i}\right)^{2} \notin S_{I}$. 
- If $m_{k j}=2$, then $m_{k i}=m_{j k}=1$ and $q_{k k} \neq-1$. The proof is similar to the previous case.

- If $m_{i k}=2, m_{i j}=0$, then $s_{i}\left(m \mathbf{e}_{k}+\mathbf{e}_{j}\right)=2 m \mathbf{e}_{i}+m \mathbf{e}_{k}+\mathbf{e}_{j}$ and $m_{k j}=0,1$. When $m_{k j}=0$, the proof is clear as above. When $m_{k j}=1$, for $j<k<i$ and considering only the quantum Serre relations, the only possible Lyndon word is $x_{j}\left(x_{k} x_{i}^{2}\right)^{2}$. But since $\left[\left[x_{i}^{2} x_{k}\right]_{c},\left[x_{i} x_{k}\right]_{c}\right]_{c}=0$, we deduce that such a word is not in $S_{I}$.

- If $m_{i k}=0, m_{i j}=1$, then $s_{i}\left(m \mathbf{e}_{k}+\mathbf{e}_{j}\right)=\mathbf{e}_{i}+m \mathbf{e}_{k}+\mathbf{e}_{j}$. If $k<i<j$, note that from $x_{k} x_{i}, x_{k}^{m_{k j}+1} x_{j} \notin S_{I}$, there are no Lyndon words of degree $\mathbf{e}_{i}+\left(m_{k j}+1\right) \mathbf{e}_{k}+\mathbf{e}_{j}$ in $S_{I}$.

- If $m_{i k}=0, m_{i j}=2$, then $s_{i}\left(m \mathbf{e}_{k}+\mathbf{e}_{j}\right)=2 \mathbf{e}_{i}+m \mathbf{e}_{k}+\mathbf{e}_{j}$, and the proof is analogous to the previous case.

- If $m_{i k}=m_{i j}=1$, then $m_{k j}=0$, and $s_{i}\left(\mathbf{e}_{k}+\mathbf{e}_{j}\right)=2 \mathbf{e}_{i}+\mathbf{e}_{k}+\mathbf{e}_{j}$, which is not in $\Delta^{+}(\mathfrak{B})$ from Lemma 5.4.

- If $m_{i k}=2, m_{i j}=1$ (it is analogous to $m_{i k}=1, m_{i j}=2$ ), then $m_{k j}=0$ and $s_{i}\left(\mathbf{e}_{k}+\mathbf{e}_{j}\right)=3 \mathbf{e}_{i}+\mathbf{e}_{k}+\mathbf{e}_{j}$. In this way we get $q_{i i} \neq-1$, and if $x_{k}<x_{i}<x_{j}$ the unique Lyndon word without $x_{i}^{2} x_{j}$ or $x_{k} x_{i}^{3}$ as factors is

$$
\begin{aligned}
& x_{k} x_{i}^{2} x_{j} x_{i}=\left(1+q_{i i}\right)^{-1} q_{i j}^{-1} x_{k} x_{i}^{3} x_{j}+\left(1+q_{i i}\right)^{-1} q_{i i}^{2} q_{i j} x_{k} x_{i} x_{j} x_{i}^{2} \\
& \in \mathrm{k}\left(x_{i} x_{k} x_{i}^{2} x_{j}\right)+\mathrm{k}\left(x_{i}^{2} x_{k} x_{i} x_{j}\right)+\mathrm{k}\left(x_{i}^{3} x_{k} x_{j}\right)+\mathrm{k}\left(x_{k} x_{i} x_{j} x_{i}^{2}\right),
\end{aligned}
$$

using the quantum Serre relations; hence there are no Lyndon words of degree $3 \mathbf{e}_{i}+\mathbf{e}_{k}+\mathbf{e}_{j}$ in $S_{I}$.

So, (5-3) holds, for each $k, j \neq i, k \neq j$.

Proof of (5-36). Assume $m_{k j}=m_{k l}=1$. We prove case by case that

$$
s_{i}\left(2 \mathbf{e}_{k}+\mathbf{e}_{j}+\mathbf{e}_{l}\right) \notin \Delta^{+}(\mathfrak{B}) .
$$

- If $m_{i j}=m_{i k}=m_{i l}=0$, then $s_{i}\left(2 \mathbf{e}_{k}+\mathbf{e}_{j}+\mathbf{e}_{l}\right)=2 \mathbf{e}_{k}+\mathbf{e}_{j}+\mathbf{e}_{l}$, so it follows from Lemma 5.4, because $2 \mathbf{e}_{k}+\mathbf{e}_{j}+\mathbf{e}_{l} \notin \Delta^{+}(\mathfrak{B})$.

- If $m_{i j} \neq 0$ (analogously, if $m_{i l} \neq 0$ ), then $m_{i k}=m_{i l}=0$, because there are no cycles in the Dynkin diagram. Then $s_{i}\left(2 \mathbf{e}_{k}+\mathbf{e}_{j}+\mathbf{e}_{l}\right)=2 \mathbf{e}_{k}+\mathbf{e}_{j}+\mathbf{e}_{l}+m_{i j} \mathbf{e}_{i}$. If we consider $x_{k}<x_{l}<x_{j}<x_{i}$, using the equalities $x_{k} x_{i}=q_{k i} x_{i} x_{k}, x_{j} x_{l}=q_{j l} x_{l} x_{j}$ and $x_{l} x_{i}=q_{l i} x_{i} x_{l}$, and also that $x_{k}^{2} x_{l}, x_{k}^{2} x_{j} \notin S_{I}$, we conclude that no possible Lyndon words of degree $2 e_{k}+e_{j}+e_{l}+m_{i j} e_{i}$ can be an element of $S_{I}$, except $x_{k} x_{l} x_{k} x_{j} x_{i}^{m_{i j}}$; but this, too, is not an element of $S_{I}$, because $x_{k} x_{l} x_{k} x_{j} \notin S_{I}$. Hence $2 \mathbf{e}_{k}+\mathbf{e}_{j}+\mathbf{e}_{l}+m_{i j} \mathbf{e}_{i} \notin \Delta^{+}(\mathfrak{B})$.

- If $m_{i k}=1$, and therefore $m_{i j}=m_{i l}=0$, then $s_{i}\left(2 \mathbf{e}_{k}+\mathbf{e}_{j}+\mathbf{e}_{l}\right)=2 \mathbf{e}_{k}+\mathbf{e}_{j}+\mathbf{e}_{l}+$ $2 m_{i k} \mathbf{e}_{i}$. If we consider $x_{l}<x_{i}<x_{k}<x_{j}$, using the equalities $x_{j} x_{i}=q_{j i} x_{i} x_{j}$, 
$x_{j} x_{l}=q_{j l} x_{l} x_{j}$ and $x_{l} x_{i}=q_{l i} x_{i} x_{l}$, and also that $x_{k}^{2} x_{l}, x_{k}^{2} x_{j} \notin S_{I}$, we discard as before all possible Lyndon words of degree $2 \mathbf{e}_{k}+\mathbf{e}_{j}+\mathbf{e}_{l}+2 m_{i k} \mathbf{e}_{i}$, except $x_{l} x_{k} x_{j} x_{k} x_{i}^{2 m_{i j}}$; but this is not an element of $S_{I}$, because $x_{k} x_{l} x_{k} x_{j} \notin S_{I}$. Thus $2 \mathbf{e}_{k}+\mathbf{e}_{j}+\mathbf{e}_{l}+2 m_{i j} \mathbf{e}_{i} \notin \Delta^{+}(\mathfrak{B})$.

- If $i=j$ (analogously, if $i=l$ ), then $s_{j}\left(2 \mathbf{e}_{k}+\mathbf{e}_{j}+\mathbf{e}_{l}\right)=2 \mathbf{e}_{k}+\mathbf{e}_{j}+\mathbf{e}_{l} \notin \Delta^{+}(\mathfrak{B})$ if $m_{j k}=1$ by Lemma 5.4, or $s_{j}\left(2 \mathbf{e}_{k}+\mathbf{e}_{j}+\mathbf{e}_{l}\right)=2 \mathbf{e}_{k}+3 \mathbf{e}_{j}+\mathbf{e}_{l} \notin \Delta^{+}(\mathfrak{B})$ if $m_{j k}=2$ by Lemma 5.5 .

- If $i=k$, then $s_{k}\left(2 \mathbf{e}_{k}+\mathbf{e}_{j}+\mathbf{e}_{l}\right)=\mathbf{e}_{j}+\mathbf{e}_{l} \notin \Delta^{+}(\mathfrak{B})$, since $m_{j l}=0$.

Also, if $\mathbf{u} \in\left\{\mathbf{e}_{k}+\mathbf{e}_{j}, \mathbf{e}_{k}+\mathbf{e}_{l}, \mathbf{e}_{k}, \mathbf{e}_{j}, \mathbf{e}_{l}\right\}$, then $\mathbf{u} \in \Delta\left(\mathfrak{B}\left(V_{i}\right)\right)$, so $s_{i}(\mathbf{u}) \in \Delta(\mathfrak{B}(V))$. The canonical surjective algebra morphisms from $T(V)$ to $\mathfrak{B}$ and $\mathfrak{B}(V)$ induce a surjective algebra morphism $\mathfrak{B} \rightarrow \mathfrak{B}(V)$, so $\Delta(\mathfrak{B}(V)) \subseteq \Delta(\mathfrak{B})$; in particular, each $s_{i}(\mathbf{u})$ lies in $\Delta(\mathfrak{B})$.

Consider a basis as in Proposition 1.11 for an order such that $x_{j}<x_{k}<x_{l}$. From Lemma 2.7, $x_{j} x_{k}, x_{k} x_{l}, x_{j} x_{k} x_{l}$ are elements of this basis, since they are not linear combinations of greater words modulo $I_{i}$, the ideal of $T\left(V_{i}\right)$ such that $s_{i}(\mathfrak{B})=$ $T\left(V_{i}\right) / I_{i}$. In the same way, $\left(x_{k} x_{l}\right)\left(x_{j} x_{k}\right), x_{l} x_{k}\left(x_{j} x_{k}\right),\left(x_{k} x_{l}\right) x_{k} x_{j}, x_{k}\left(x_{j} x_{k} x_{l}\right), x_{l} x_{k}^{2} x_{j}$ (if $x_{k}^{2} \neq 0$ ) are elements of this basis, where the parenthesis indicates the Lyndon decomposition as nonincreasing products of Lyndon words. Also, $x_{j} x_{l}, x_{j} x_{k}^{2}, x_{k}^{2} x_{l}$ are not in this basis, by (5-3). By the relation (2-15) between Hilbert series and the fact that $2 \mathbf{e}_{k}+\mathbf{e}_{j}+\mathbf{e}_{l} \notin s_{i}\left(\Delta^{+}(\mathfrak{B})\right)$, we note that $x_{j} x_{k} x_{l} x_{k}$ is not an element of the basis. Thus this word is a linear combination of greater words. By Lemma 5.4, this implies that (5-4) holds in $s_{i}(\mathfrak{B})$.

Proof of (5-37). As before, we prove first that $s_{i}\left(3 \mathbf{e}_{k}+2 \mathbf{e}_{j}\right) \notin \Delta^{+}(\mathfrak{B})$ case by case:

- If $m_{i k}=m_{i j}=0$, then $s_{i}\left(3 \mathbf{e}_{k}+2 \mathbf{e}_{j}\right)=3 \mathbf{e}_{k}+2 \mathbf{e}_{j} \notin \Delta^{+}(\mathfrak{B})$ by assumption.

- If $m_{i k}=0, m_{i j}=1$, then $s_{i}\left(3 \mathbf{e}_{k}+2 \mathbf{e}_{j}\right)=2 \mathbf{e}_{i}+3 \mathbf{e}_{k}+2 \mathbf{e}_{j}$. If we consider an order such that $x_{k}<x_{i}<x_{j}$, a Lyndon word of degree $2 e_{i}+3 e_{k}+2 e_{j}$ in $S_{I}$ begins with $x_{k}$, and $x_{k} x_{i}$ is not a factor, because $x_{k} x_{i}=q_{k i} x_{i} x_{j}$. Thus the possible Lyndon words with these conditions are $x_{k}^{2} x_{j} x_{i} x_{k} x_{j} x_{i}$ and $x_{k}^{2} x_{j} x_{k} x_{j} x_{i}^{2}$; the first is not in $S_{I}$ because from (5-4) for $j, k, i$ we can express $x_{j} x_{i} x_{k} x_{j}$ as a linear combination of greater words, and the second is not in $S_{I}$ because $x_{k}^{2} x_{j} x_{k} x_{j} \notin S_{I}$.

- If $m_{i k}=1, m_{i j}=0$, then $s_{i}\left(3 \mathbf{e}_{k}+2 \mathbf{e}_{j}\right)=3 \mathbf{e}_{i}+3 \mathbf{e}_{k}+2 \mathbf{e}_{j}$. If we consider an order such that $x_{j}<x_{i}<x_{k}$, a Lyndon word of degree $3 e_{i}+3 e_{k}+2 e_{j}$ in $S_{I}$ begins with $x_{j}$, and $x_{j} x_{i}$ is not a factor. Using that also $x_{i}^{2} x_{k}, x_{j}^{2} x_{k} \notin S_{I}$, the possible Lyndon word under these conditions is $x_{j} x_{k} x_{i} x_{j} x_{k} x_{i} x_{k} x_{i}$. But from the condition on the $m_{r s}$, we are in cases $C_{\theta}$ or $F_{4}$, and we use that $\left(\operatorname{ad} x_{i}\right)^{2} x_{k}=0$, $q_{i i} \neq-1$ to replace $x_{i} x_{k} x_{i}$ by a linear combination of $x_{i}^{2} x_{k}$ and $x_{k} x_{i}^{2}$, and also use $x_{j} x_{i}=q_{j i} x_{i} x_{j}$, so we conclude that $x_{j} x_{k} x_{i} x_{j} x_{k} x_{i} x_{k} x_{i} \notin S_{I}$.

- If $i=j$, then $s_{j}\left(3 \mathbf{e}_{k}+2 \mathbf{e}_{j}\right)=3 \mathbf{e}_{k}+\mathbf{e}_{j} \notin \Delta^{+}(\mathfrak{B})$, since $m_{k j}=2$. 
- If $i=k$, then $s_{k}\left(3 \mathbf{e}_{k}+2 \mathbf{e}_{j}\right)=\mathbf{e}_{k}+2 \mathbf{e}_{j} \notin \Delta^{+}(\mathfrak{B})$, since $m_{j k}=1$.

If $\mathbf{v} \in\left\{\mathbf{e}_{k}+\mathbf{e}_{j}, 2 \mathbf{e}_{k}+\mathbf{e}_{j}, \mathbf{e}_{k}, \mathbf{e}_{j}\right\}$, then $\mathbf{v} \in \Delta\left(\mathfrak{B}\left(V_{i}\right)\right)$, so $s_{i}(\mathbf{v}) \in \Delta(\mathfrak{B}(V))$. Since $\Delta(\mathfrak{B}(V)) \subseteq \Delta(\mathfrak{B})$; in particular, each $\mathbf{v}$ lies in $s_{i}(\Delta(\mathfrak{B}))$.

As in (a), consider a basis as in Proposition 1.11 for an order such that $x_{k}<x_{j}$. In a similar way, $x_{k} x_{j}, x_{k}^{2} x_{j}$ are elements of this basis, but $x_{k}^{3} x_{j}$ and $x_{k} x_{j}^{2}$ are not in this basis by (5-3). By Lemma 2.7, $\left(x_{k} x_{j}\right)\left(x_{k}^{2} x_{j}\right), x_{j}\left(x_{k}^{2} x_{j}\right) x_{k},\left(x_{k} x_{j}\right)^{2} x_{k}, x_{j}\left(x_{k} x_{j}\right) x_{k}^{2}$, $x_{j}^{2} x_{k}^{3}$ (the last if $x_{j}^{2}, x_{k}^{3} \neq 0$ ) are not linear combinations of greater words modulo $I_{i}$, so they are elements of the chosen basis. By the relation (2-15) between Hilbert series and the fact that $3 \mathbf{e}_{k}+2 \mathbf{e}_{j} \notin s_{i}\left(\Delta^{+}(\mathfrak{B})\right)$, the Lyndon word $x_{k}^{2} x_{j} x_{k} x_{j}$ is not an element of the basis. Thus this word is a linear combination of greater words, and by Lemma 5.5, this implies that (5-6) holds in $s_{i}(\mathfrak{B})$.

Proof of (5-38). We prove case by case that

$$
s_{i}\left(3 \mathbf{e}_{k}+2 \mathbf{e}_{j}+\mathbf{e}_{l}\right) \notin \Delta^{+}(\mathfrak{B}) .
$$

- If $m_{i k}=m_{i j}=m_{i l}=0$, then $s_{i}\left(3 \mathbf{e}_{k}+2 \mathbf{e}_{j}+\mathbf{e}_{l}\right)=3 \mathbf{e}_{k}+2 \mathbf{e}_{j}+\mathbf{e}_{l}$, and this is not in $\Delta^{+}(\mathfrak{B})$ by Lemma 5.6.

- If $i \neq j, k, l$ and $m_{i k} \neq 0$, the only possibility is $m_{i k}=m_{k i}=1$, so $V$ is of type $F_{4}$. Thus $s_{i}\left(3 \mathbf{e}_{k}+2 \mathbf{e}_{j}+\mathbf{e}_{l}\right)=3 \mathbf{e}_{i}+3 \mathbf{e}_{k}+2 \mathbf{e}_{j}+\mathbf{e}_{l}$. For the order $x_{l}<x_{j}<x_{k}<x_{i}$, the only possible Lyndon word without the factors $x_{l} x_{j}^{2}, x_{l} x_{k}, x_{l} x_{i}, x_{j}^{2} x_{k}, x_{j} x_{i}$, $x_{k} x_{i}^{2}, x_{k}^{2} x_{i}$ is $x_{l} x_{j} x_{k} x_{i} x_{j} x_{k} x_{i} x_{k} x_{i}$. Using the quantum Serre relations and the fact that $q_{i i}=q_{k k} \neq-1$, we see that this Lyndon word is not in $S_{I}$. Thus $3 \mathbf{e}_{i}+3 \mathbf{e}_{k}+2 \mathbf{e}_{j}+\mathbf{e}_{l} \notin \Delta^{+}(\mathfrak{B})$.

- $i \neq j, k, l$ and $m_{i j} \neq 0$ : there are no standard braided vector spaces with these values.

- If $i \neq j, k, l$ and $m_{i l} \neq 0$, the unique possibility is $m_{i l}=m_{l i}=1$. In this case $s_{i}\left(3 \mathbf{e}_{k}+2 \mathbf{e}_{j}+\mathbf{e}_{l}\right)=3 \mathbf{e}_{k}+2 \mathbf{e}_{j}+\mathbf{e}_{l}+\mathbf{e}_{i}$. If we consider $x_{k}<x_{j}<x_{l}<x_{i}$, the only possible Lyndon word of this degree without the factors $x_{k} x_{l}, x_{k} x_{i}, x_{j} x_{i}$, $x_{k}^{3} x_{j}, x_{k} x_{j}^{2}$ is $x_{k}^{2} x_{j} x_{l} x_{i} x_{k} x_{i}$. But by assumption,

$$
\left[\left[x_{k}^{2} x_{j} x_{l}\right]_{c},\left[x_{k} x_{j}\right]_{c}\right]_{c}=\left[x_{i},\left[x_{k} x_{j}\right]_{c}\right]_{c}=0,
$$

so $\left[x_{k}^{2} x_{j} x_{l} x_{i} x_{k} x_{i}\right]_{c}=\left[\left[x_{k}^{2} x_{j} x_{l} x_{i}\right]_{c},\left[x_{k} x_{j}\right]_{c}\right]_{c}=0$, and $x_{k}^{2} x_{j} x_{l} x_{i} x_{k} x_{i} \notin S_{I}$.

- If $i=k$, then $s_{i}\left(3 \mathbf{e}_{i}+2 \mathbf{e}_{j}+\mathbf{e}_{l}\right)=\mathbf{e}_{i}+2 \mathbf{e}_{j}+\mathbf{e}_{l} \notin \Delta^{+}(\mathfrak{B})$, by Lemma 5.4.

- If $i=j$, then $s_{i}\left(3 \mathbf{e}_{k}+2 \mathbf{e}_{i}+\mathbf{e}_{l}\right)=3 \mathbf{e}_{k}+2 \mathbf{e}_{i}+\mathbf{e}_{l} \notin \Delta^{+}(\mathfrak{B})$, by Lemma 5.6.

- If $i=k$, then $s_{i}\left(3 \mathbf{e}_{k}+2 \mathbf{e}_{j}+\mathbf{e}_{i}\right)=\mathbf{e}_{k}+2 \mathbf{e}_{j}+\mathbf{e}_{i} \notin \Delta^{+}(\mathfrak{B})$, as before.

Now, if $\mathbf{w} \in\left\{\mathbf{e}_{k}, \mathbf{e}_{j}, \mathbf{e}_{l}, \mathbf{e}_{k}+\mathbf{e}_{j}, \mathbf{e}_{k}+\mathbf{e}_{j}+\mathbf{e}_{l}, 2 \mathbf{e}_{k}+\mathbf{e}_{j}, 2 \mathbf{e}_{k}+\mathbf{e}_{j}+\mathbf{e}_{l}, 2 \mathbf{e}_{k}+2 \mathbf{e}_{j}+\mathbf{e}_{l}\right\}$, then $\mathbf{w} \in \Delta\left(\mathfrak{B}\left(V_{i}\right)\right)$, so $s_{i}(\mathbf{w}) \in \Delta(\mathfrak{B}(V))$, hence $s_{i}(\mathbf{w}) \in \Delta(\mathfrak{B})$. 
Consider a basis as in Proposition 1.11 for an order such that $x_{k}<x_{j}<x_{l}$. Then $x_{j} x_{k}$ and $x_{k} x_{l}$ are elements of this basis. We know that $x_{k} x_{l}, x_{k}^{3} x_{j}, x_{k} x_{j}^{2}$, $x_{k} x_{j} x_{l} x_{k}, x_{k}^{2} x_{j} x_{k} x_{j}$ are not elements of the basis, since (5-3), (5-4) and (5-6) hold in $\mathfrak{B}$. By Lemma 2.7, the relation (2-15) between Hilbert series and the fact that $3 \mathbf{e}_{k}+2 \mathbf{e}_{j}+\mathbf{e}_{l} \notin s_{i}\left(\Delta^{+}(\mathfrak{B})\right)$, the Lyndon word $x_{k}^{2} x_{j} x_{l} x_{k} x_{j}$ is not an element of the basis. Thus this word is a linear combination of greater words. By Lemma 5.6, this implies that (5-8) holds in $s_{i}(\mathfrak{B})$.

This concludes the proof of Lemma 5.23. Note also that $s_{i}(\mathfrak{B})$ is of the same type as $\mathfrak{B}$.

Let $V$ be of a type different from $G_{2}$. We define the algebra $\hat{\mathfrak{B}}(V):=T(V) / \mathfrak{I}(V)$, where $\mathfrak{I}(V)$ is the two-sided ideal of $T(V)$ generated by

- $\left(\operatorname{ad}_{c} x_{k}\right)^{m_{k j}+1} x_{j}, k \neq j$;

- $\left[\left(\operatorname{ad}_{c} x_{j}\right)\left(\operatorname{ad}_{c} x_{k}\right) x_{l}, x_{k}\right]_{c}, l \neq k \neq j, q_{k k}=-1, m_{k j}=m_{k l}=1$;

- $\left[\left(\operatorname{ad}_{c} x_{k}\right)^{2} x_{j},\left(\operatorname{ad}_{c} x_{k}\right) x_{j}\right]_{c}, k \neq j, q_{k k} \in \mathbb{G}_{3}$ or $q_{j j}=-1, m_{k j}=2, m_{j k}=1$;

- $\left[\left(\operatorname{ad}_{c} x_{k}\right)^{2}\left(\operatorname{ad}_{c} x_{j}\right) x_{l},\left(\operatorname{ad}_{c} x_{k}\right) x_{j}\right]_{c}, k \neq j \neq l, q_{k k} \in \mathbb{G}_{3}$ or $q_{j j}=-1, m_{k j}=2$, $m_{j k}=m_{j l}=1$.

(Compare with the definitions in Section 4 of [Andruskiewitsch and Schneider 2002a].) Since $V$ is of Cartan type, $\mathfrak{I}(V)$ is a Hopf ideal, by Lemmas 5.7-5.9. Since $\mathfrak{I}(V)$ also is $\mathbb{Z}^{\theta}$-homogeneous, we have $\mathfrak{I}(V) \in \mathfrak{S}$.

By Lemmas 5.4-5.6, the canonical epimorphism $T(V) \rightarrow \mathfrak{B}(V)$ induces an epimorphism of braided graded Hopf algebras

$$
\pi_{V}: \hat{\mathfrak{B}}(V) \rightarrow \mathfrak{B}(V) .
$$

Also, $\hat{\mathfrak{B}}(V)$ satisfies the conditions in Theorem 2.6 for each $i \in\{1, \ldots, \theta\}$, so we can transform it.

Lemma 5.24. With the notation above, $s_{i}(\hat{\mathfrak{B}}(V)) \cong \hat{\mathfrak{B}}\left(V_{i}\right)$.

Proof. By Lemma 5.23, the relations defining $\Im\left(V_{i}\right)$ are satisfied in $s_{i}(\hat{\mathfrak{B}}(V))$. Thus the canonical projections from $T\left(V_{i}\right)$ onto $\hat{\mathfrak{B}}\left(V_{i}\right)$ and $s_{i}(\hat{\mathfrak{B}}(V))$ induce a surjective algebra map $\hat{\mathfrak{B}}\left(V_{i}\right) \rightarrow s_{i}(\hat{\mathfrak{B}}(V))$. Conversely, each relation defining $\Im(V)$ is satisfied in $s_{i}\left(\hat{\mathfrak{B}}\left(V_{i}\right)\right)$, so we have the following situation:

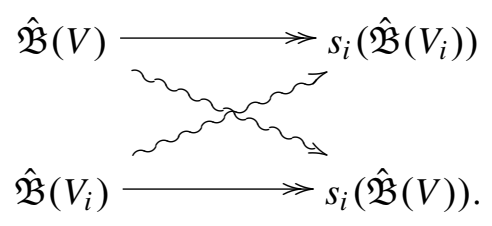


From the relation (2-15) between Hilbert series, we have, for each $\mathbf{u} \in \mathbb{N}^{\theta}$,

$$
\operatorname{dim} s_{i}(\hat{\mathfrak{B}}(V))^{\mathbf{u}}=\sum_{\substack{k \in \mathbb{N}: \mathbf{u}-k \mathbf{e}_{i} \in \mathbb{N}^{\theta} \\ s_{i}\left(\mathbf{u}-k \mathbf{e}_{i}\right) \in \mathbb{N}^{\theta}}} \operatorname{dim} \hat{\mathfrak{B}}(V)^{s_{i}\left(\mathbf{u}-k \mathbf{e}_{i}\right)},
$$

and a analogous relation for $\operatorname{dim} s_{i}\left(\hat{\mathfrak{B}}\left(V_{i}\right)\right)^{\mathbf{u}}$. But in view of the previous surjections we have

$$
\operatorname{dim} s_{i}(\hat{\mathfrak{B}}(V))^{\mathbf{u}} \leq \operatorname{dim} \hat{\mathfrak{B}}\left(V_{i}\right)^{\mathbf{u}}, \quad \operatorname{dim} s_{i}\left(\hat{\mathfrak{B}}\left(V_{i}\right)\right)^{\mathbf{u}} \leq \operatorname{dim} \hat{\mathfrak{B}}(V)^{\mathbf{u}},
$$

for each $\mathbf{u} \in \mathbb{N}^{\theta}$. Since $s_{i}^{2}=\mathrm{id}$, each of these inequalities is in fact an equality, and $s_{i}(\hat{\mathfrak{B}}(V))=\hat{\mathfrak{B}}\left(V_{i}\right)$.

We are now able to prove one of the main results of this paper.

Theorem 5.25. Let $V$ be a braided vector space of Cartan type, of dimension $\theta$, and $C=\left(a_{i j}\right)_{i, j \in\{1, \ldots, \theta\}}$ the corresponding finite Cartan matrix, where $a_{i j}:=-m_{i j}$.

The Nichols algebra $\mathfrak{B}(V)$ is presented by the generators $x_{i}$, for $1 \leq i \leq \theta$, and the relations

$$
\begin{gathered}
x_{\alpha}^{N_{\alpha}}=0, \quad \alpha \in \Delta^{+}, \\
\operatorname{ad}_{c}\left(x_{k}\right)^{1-a_{k j}}\left(x_{j}\right)=0, \quad k \neq j .
\end{gathered}
$$

If there exist $j \neq k \neq l$ such that $m_{k j}=m_{k l}=1, q_{k k}=-1$, then

$$
\left[\left(\operatorname{ad} x_{k}\right) x_{j},\left(\operatorname{ad} x_{k}\right) x_{l}\right]_{c}=0 .
$$

If there exist $k \neq j$ such that $m_{k j}=2, m_{j k}=1, q_{k k} \in \mathbb{G}_{3}$ or $q_{j j}=-1$, then

$$
\left[\left(\operatorname{ad} x_{k}\right)^{2} x_{j},\left(\operatorname{ad} x_{k}\right) x_{j}\right]_{c}=0 .
$$

If there exist $k \neq j \neq l$ such that $m_{k j}=2, m_{j k}=m_{j l}=1, q_{k k} \in \mathbb{G}_{3}$ or $q_{j j}=-1$, then

$$
\left[\left(\operatorname{ad} x_{k}\right)^{2}\left(\operatorname{ad} x_{j}\right) x_{l},\left(\operatorname{ad} x_{k}\right) x_{j}\right]_{c}=0 .
$$

If $\theta=2, V$ if of type $G_{2}$, and $q_{11} \in \mathbb{G}_{4}$ or $q_{22}=-1$, then

$$
\begin{aligned}
{\left[\left(\operatorname{ad} x_{1}\right)^{3} x_{2},\left(\operatorname{ad} x_{1}\right)^{2} x_{2}\right]_{c} } & =0, \\
{\left[x_{1},\left[x_{1}^{2} x_{2} x_{1} x_{2}\right]_{c}\right]_{c} } & =0, \\
{\left[\left[x_{1}^{2} x_{2} x_{1} x_{2}\right]_{c},\left[x_{1} x_{2}\right]_{c}\right]_{c} } & =0, \\
{\left[\left[x_{1}^{2} x_{2}\right]_{c},\left[x_{1}^{2} x_{2} x_{1} x_{2}\right]_{c}\right]_{c} } & =0 .
\end{aligned}
$$

The following elements constitute a basis of $\mathfrak{B}(V)$ : $x_{\beta_{1}}^{h_{1}} x_{\beta_{2}}^{h_{2}} \ldots x_{\beta_{P}}^{h_{P}}$, where $0 \leq h_{j} \leq N_{\beta_{j}}-1$, if $\beta_{j} \in S_{I}$, for $1 \leq j \leq P$. 
Proof. We may assume that $C$ is connected. For $V$ of type $G_{2}$, the result was proved in Theorem 5.22. So we can assume $m_{k j} \neq 3, k \neq j$.

The statement about the PBW basis was proved in Corollary 4.2; see the definition of the $x_{\alpha}$ in Section 4B.

Consider the images of the $x_{\alpha}$ in $\hat{\mathfrak{B}}(V)$; they correspond in $\mathfrak{B}(V)$ with the $x_{\alpha}$, and are PBW generators for a basis constructed as in Theorem 1.12, considering the same order in the letters. As we observed in (5-39), there exists a surjective morphism of braided Hopf algebras $\hat{\mathfrak{B}}(V) \rightarrow \mathfrak{B}(V)$, so

$$
\Delta(\mathfrak{B}(V)) \subseteq \Delta(\hat{\mathfrak{B}}(V)) .
$$

Also, $\hat{\mathfrak{B}}(V)$ satisfies the conditions in Theorem 2.6 for each $i \in\{1, \ldots, \theta\}$, so we can transform it. By Lemma 5.24, the new algebra is $\hat{\mathfrak{B}}\left(V_{i}\right)$, so we can continue. Consider the sets

$$
\hat{\Delta}:=\bigcup\left\{\Delta\left(s_{i_{1}} \ldots s_{i_{k}} \hat{\mathfrak{B}}\right): k \in \mathbb{N}, 1 \leq i_{1}, \ldots, i_{k} \leq \theta\right\}, \quad \hat{\Delta}^{+}:=\Delta \cap \mathbb{N}^{\theta} ;
$$

$\hat{\Delta}$ is invariant by the $s_{i}$. Also, $\Delta(\mathfrak{B}(V)) \subseteq \Delta$, and

$$
\Delta\left(s_{i_{1}} \ldots s_{i_{k}} \hat{\mathfrak{B}}(V)\right)=s_{i_{1}} \ldots s_{i_{k}} \Delta(\hat{\mathfrak{B}}(V)) .
$$

Consider $\alpha \in \hat{\Delta}^{+} \backslash \Delta^{+}(\mathfrak{B}(V))$. Suppose that $\alpha$ is not of the form $m \alpha_{i}$ for $m \in \mathbb{N}$ and $i \in\{1, \ldots, \theta\}$, and that it is of minimal height among such roots. For each $s_{i}$, since $\alpha$ is not a multiple of $\alpha_{i}$, we have $s_{i}(\alpha) \in \Delta^{+} \backslash \Delta^{+}(\mathfrak{B}(V))$; hence $\operatorname{deg} s_{i}(\alpha)-\operatorname{deg} \alpha \geq 0$. But $\alpha=\sum_{i=1}^{\theta} b_{i} \mathbf{e}_{i}$, so $\sum_{i=1}^{\theta} b_{i} a_{i j} \leq 0$, and since $b_{i} \geq 0$, we have $\sum_{i, j=1}^{\theta} b_{i} a_{i j} b_{j} \leq 0$. This contradicts the fact that $\left(a_{i j}\right)$ is definite positive, and $\left(b_{i}\right) \geq 0,\left(b_{i}\right) \neq 0$.

Also, $m \mathbf{e}_{i} \in \Delta^{+}(\hat{\mathfrak{B}}) \Longleftrightarrow m=N_{\mathbf{e}_{i}}$ or $m=1$, since $x_{i}^{N_{\mathbf{e}_{i}}} \neq 0$. Hence

$$
\Delta(\hat{\mathfrak{B}}(V))=\Delta(\mathfrak{B}(V)) \cup\left\{N_{\alpha} \alpha: \alpha \in \Delta(\mathfrak{B}(V))\right\} .
$$

This follows since by Corollary 4.2 each $\alpha \in \Delta^{+}(\mathfrak{B}(V))$ is of the form

$$
\alpha=s_{i_{1}} \cdots s_{i_{m}}\left(\mathbf{e}_{j}\right), \quad i_{1}, \ldots, i_{m}, j \in\{1, \ldots, \theta\} .
$$

Now, $N_{\mathbf{e}_{j}} \mathbf{e}_{j} \in \Delta(\hat{\mathfrak{B}}(V))$, so

$$
N_{\alpha} \alpha=N_{\mathbf{e}_{j}} \alpha=s_{i_{1}} \ldots s_{i_{m}}\left(N_{\mathbf{e}_{j}} \mathbf{e}_{j}\right) \in \Delta(\hat{\mathfrak{B}}(V)) .
$$

Also, each degree $N_{\alpha} \alpha$ has multiplicity one in $\Delta(\hat{\mathfrak{B}}(V))$.

Suppose there exist Lyndon words of degree $N_{\alpha} \alpha$, and consider one such word $u$ of minimal height. Let $u=v w$ be a Shirshov decomposition thereof, and put

$$
\beta:=\operatorname{deg} v, \gamma:=\operatorname{deg} w \in \Delta^{+}(\hat{\mathfrak{B}}(V)) .
$$


By the preceding assumption, $\beta, \gamma \in \Delta^{+}(\mathfrak{B}(V))$. Write

$$
\alpha=\sum_{k=1}^{\theta} a_{k} \mathbf{e}_{k}, \quad \beta=\sum_{k=1}^{\theta} b_{k} \mathbf{e}_{k}, \quad \gamma=\sum_{k=1}^{\theta} c_{k} \mathbf{e}_{k},
$$

so $N_{\alpha} a_{k}=b_{k}+c_{k}$, for each $k \in\{1, \ldots, \theta\}$. We can assume, by taking a subdiagram if necessary, that $a_{1}, a_{\theta} \neq 0$.

Now, if $V$ is of type $F_{4}$ and $\beta=2 \mathbf{e}_{1}+3 \mathbf{e}_{2}+4 \mathbf{e}_{3}+3 \mathbf{e}_{4}$, then $c_{1}=0, a_{1}=1$, $N_{\alpha}=2$, or $a_{1}=c_{1}=1, N_{\alpha}=3$, since $\alpha, \gamma \neq \beta$.

- If $N_{\alpha}=3$, then $3 a_{2}=3+c_{2}$. Hence $c_{2}=0$, so $c_{3}=c_{4}=0$, or $c_{2}=3$, and $c_{3}=4, c_{4}=2$. But in both cases we have a contradiction to $\alpha \in \mathbb{N}^{4}$.

- If $N_{\alpha}=2, c_{1}=0$, then $c_{2}$ and $c_{4}$ are odd, and $c_{3}$ is even and nonzero. The only possibility is $\gamma=\mathbf{e}_{2}+2 \mathbf{e}_{3}+\mathbf{e}_{4}$, so $\alpha=\mathbf{e}_{1}+2 \mathbf{e}_{2}+3 \mathbf{e}_{3}+2 \mathbf{e}_{4}$. But $q_{\alpha}=q \neq-1$, so $N_{\alpha} \neq 2$, which is a contradiction.

Thus we can assume $b_{1}, c_{1} \leq 1$ or $b_{\theta}, c_{\theta} \leq 1$, so $a_{1}=b_{1}=c_{1}=1$ or $a_{\theta}=b_{\theta}=$ $c_{\theta}=1$; in both cases, $N_{\alpha}=2$. For each possible $\beta$ with $b_{1} \neq 0$ (by the assumption that $a_{1} \neq 0$, we have $b_{1} \neq 0$ or $c_{1} \neq 0$ ), we look for $\gamma$ such that $\beta+\gamma$ has even coordinates. In types $A, D$ and $E$ there are no such pairs of roots. As for the other types:

- $B_{\theta}: \beta=\mathbf{v}_{i \theta}, \gamma=\mathbf{u}_{i+1, \theta}$. Then $\alpha=\mathbf{u}_{1 \theta}$, but $q_{\alpha}=q_{11} \neq-1$, which is a contradiction.

- $C_{\theta}: \beta=\mathbf{w}_{11}, \gamma=\mathbf{e}_{\theta}$. Then $\alpha=\mathbf{u}_{1 \theta}$, but $q_{\alpha}=q_{\theta \theta} \neq-1$, which is a contradiction.

- $F_{4}: \beta=\mathbf{e}_{1}+\mathbf{e}_{2}+2 \mathbf{e}_{3}+2 \mathbf{e}_{4}, \gamma=\mathbf{e}_{1}+\mathbf{e}_{2}$, or $\beta=\mathbf{e}_{1}+2 \mathbf{e}_{2}+2 \mathbf{e}_{3}+2 \mathbf{e}_{4}, \gamma=\mathbf{e}_{1}$. In both cases, $\alpha=\mathbf{e}_{1}+\mathbf{e}_{2}+\mathbf{e}_{3}+\mathbf{e}_{4}$, but $q_{\alpha}=q \neq-1$, which is a contradiction.

Thus each root $N_{\alpha} \alpha$ corresponds to $x_{\alpha}^{N_{\alpha}}$, and each $x_{\alpha}$ has infinite height, as before. The elements

$$
x_{\beta_{1}}^{h_{1}} x_{\beta_{2}}^{h_{2}} \ldots x_{\beta_{P}}^{h_{P}}, \quad \text { where } 0 \leq h_{j}<\infty \text {, if } \beta_{j} \in S_{I} \text {, for } 1 \leq j \leq P,
$$

form a basis of $\hat{\mathfrak{B}}(V)$ as a vector space.

Now let $\bar{I}(V)$ be the ideal of $T(V)$ generated by the relations (5-41)-(5-44) and (5-40). We have $\Im(V) \subseteq \bar{I}(V) \subseteq I(V)$, so the corresponding projections induce a surjective morphism of algebras $\phi: \mathfrak{B} \rightarrow \mathfrak{B}(V)$, where $\mathfrak{B}:=T(V) / \bar{I}(V)$ :

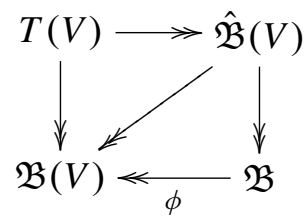


Also, the elements

$$
x_{\beta_{1}}^{h_{1}} x_{\beta_{2}}^{h_{2}} \ldots x_{\beta_{P}}^{h_{P}}, \quad \text { where } 0 \leq h_{j}<N_{\beta_{j}} \text {, if } \beta_{j} \in S_{I} \text {, for } 1 \leq j \leq P,
$$

generate $\mathfrak{B}$ as a vector space, because they correspond to images of generators of $\hat{\mathfrak{B}}(V)$ and are nonzero (as before, each nonincreasing product of hyperwords such that $h_{j} \geq N_{\beta_{j}}$ is zero in $\mathfrak{B}$ ). But $\phi$ is surjective, and the corresponding images of these elements form a basis of $\mathfrak{B}(V)$, so $\phi$ is an isomorphism.

\section{Acknowledgments}

I thank Nicolás Andruskiewitsch for his guidance, his important suggestions and his careful reading of this work. I also thank the referee for many useful comments, especially the suggestion to shorten the proofs of Lemma 2.4 and Theorem 4.1.

\section{References}

[Andersen et al. 1994] H. H. Andersen, J. C. Jantzen, and W. Soergel, Representations of quantum groups at a pth root of unity and of semisimple groups in characteristic $p$ : independence of $p$, Astérisque 220, Soc. Math. France, Paris, 1994. MR 95j:20036 Zbl 0802.17009

[Andruskiewitsch 2002] N. Andruskiewitsch, "About finite dimensional Hopf algebras", pp. 1-57 in Quantum symmetries in theoretical physics and mathematics (Bariloche, 2000), edited by R. Coquereaux et al., Contemp. Math. 294, Amer. Math. Soc., Providence, RI, 2002. MR 2003f:16059 Zbl 1135.16306

[Andruskiewitsch and Angiono 2008] N. Andruskiewitsch and I. Angiono, "On Nichols algebras with generic braiding", pp. 47-64 in Modules and comodules (Porto, Portugal, 2006), edited by T. Brzeziński et al., Birkhäuser, Basel, 2008.

[Andruskiewitsch and Dăscălescu 2005] N. Andruskiewitsch and S. Dăscălescu, "On finite quantum groups at -1”, Algebr. Represent. Theory 8:1 (2005), 11-34. MR 2006a:16061 Zbl 1136.16032

[Andruskiewitsch and Schneider 1998] N. Andruskiewitsch and H.-J. Schneider, "Lifting of quantum linear spaces and pointed Hopf algebras of order $p^{3}$, J. Algebra 209:2 (1998), 658-691. MR 99k:16075 Zbl 0919.16027

[Andruskiewitsch and Schneider 2000] N. Andruskiewitsch and H.-J. Schneider, "Finite quantum groups and Cartan matrices", Adv. Math. 154:1 (2000), 1-45. MR 2001g:16070 Zbl 1007.16027

[Andruskiewitsch and Schneider 2002a] N. Andruskiewitsch and H.-J. Schneider, "Finite quantum groups over abelian groups of prime exponent”, Ann. Sci. École Norm. Sup. (4) 35:1 (2002), 1-26. MR 2003a:16055 Zbl 1007.16028

[Andruskiewitsch and Schneider 2002b] N. Andruskiewitsch and H.-J. Schneider, "Pointed Hopf algebras", pp. 1-68 in New directions in Hopf algebras, edited by S. Montgomery and H.-J. Schneider, Math. Sci. Res. Inst. Publ. 43, Cambridge Univ. Press, Cambridge, 2002. MR 2003e:16043 Zbl 1011.16025

[Andruskiewitsch and Schneider 2004] N. Andruskiewitsch and H.-J. Schneider, "A characterization of quantum groups", J. Reine Angew. Math. 577 (2004), 81-104. MR 2005i:16083 Zbl 1084.16027

[Andruskiewitsch and Schneider 2005] N. Andruskiewitsch and H.-J. Schneider, "On the classification of finite-dimensional pointed Hopf algebras", preprint, 2005. To appear in Ann. Math. arXiv math.QA/0502157 
[Andruskiewitsch et al. 2008] N. Andruskiewitsch, I. Heckenberger, and H.-J. Schneider, "The Nichols algebra of a semisimple Yetter-Drinfeld module", preprint, 2008. arXiv 0803.2430

[Bourbaki 1968] N. Bourbaki, Groupes et algèbres de Lie, chap. IV-VI, Actualités Scientifiques et Industrielles 1337, Hermann, Paris, 1968. Zbl 0186.33001

[De Concini and Procesi 1993] C. De Concini and C. Procesi, "Quantum groups", pp. 31-140 in Dmodules, representation theory, and quantum groups (Venice, 1992), edited by G. Zampieri and A. D’Agnolo, Lecture Notes in Math. 1565, Springer, Berlin, 1993. MR 95j:17012 Zbl 0795.17005

[Drinfel'd 1987] V. G. Drinfel'd, “Quantum groups”, pp. 798-820 in Proceedings of the International Congress of Mathematicians (Berkeley, 1986), vol. 1, edited by A. M. Gleason, Amer. Math. Soc., Providence, RI, 1987. MR 89f:17017 Zbl 0667.16003

[Graña 2000] M. Graña, "On Nichols algebras of low dimension”, pp. 111-134 in New trends in Hopf algebra theory (La Falda, Argentina, 1999), edited by N. Andruskiewitsch et al., Contemp. Math. 267, Amer. Math. Soc., Providence, RI, 2000. MR 2001j:16059 Zbl 0974.16031

[Heckenberger 2006a] I. Heckenberger, "Classification of arithmetic root systems", preprint, 2006. arXiv math.QA/0605795

[Heckenberger 2006b] I. Heckenberger, "The Weyl groupoid of a Nichols algebra of diagonal type", Invent. Math. 164:1 (2006), 175-188. MR 2007e:16047

[Heckenberger 2007] I. Heckenberger, "Examples of finite-dimensional rank 2 Nichols algebras of diagonal type", Compos. Math. 143:1 (2007), 165-190. MR 2008b:16070

[Heckenberger 2008] I. Heckenberger, "Rank 2 Nichols algebras with finite arithmetic root system", Algebr. Represent. Theory 11:2 (2008), 115-132. MR 2009a:16080

[Jimbo 1985] M. Jimbo, “A $q$-difference analogue of $U(\mathfrak{g})$ and the Yang-Baxter equation", Lett. Math. Phys. 10:1 (1985), 63-69. MR 86k:17008 Zbl 0587.17004

[Kharchenko 1999] V. K. Kharchenko, "A quantum analogue of the Poincaré-Birkhoff-Witt theorem”, Algebra i Logika 38:4 (1999), 476-507. In Russian; translated in Algebra and Logic 38:4 (1999), 259-276. MR 2001f:16075 Zbl 0936.16034

[Lalonde and Ram 1995] P. Lalonde and A. Ram, "Standard Lyndon bases of Lie algebras and enveloping algebras", Trans. Amer. Math. Soc. 347:5 (1995), 1821-1830. MR 95h:17013 Zbl 0833.17003

[Lusztig 1990a] G. Lusztig, "Finite-dimensional Hopf algebras arising from quantized universal enveloping algebra”, J. Amer. Math. Soc. 3:1 (1990), 257-296. MR 91e:17009 Zbl 0695.16006

[Lusztig 1990b] G. Lusztig, "Quantum groups at roots of 1", Geom. Dedicata 35:1-3 (1990), 89113. MR 91j:17018 Zbl 0714.17013

[Lusztig 1993] G. Lusztig, Introduction to quantum groups, Progress in Math. 110, Birkhäuser, Boston, 1993. MR 94m:17016 Zbl 0788.17010

[Montgomery 1993] S. Montgomery, Hopf algebras and their actions on rings, CBMS Regional Conf. Series in Math. 82, Amer. Math. Soc., Providence, 1993. MR 94i:16019 Zbl 0793.16029

[Rosso 1999] M. Rosso, "Lyndon words and universal R-matrices", lecture delivered at the Math. Sci. Res. Inst., Berkeley, October 26, 1999, Available at http://www.msri.org/publications/ln/ msri/1999/hopfalg/rosso/1/index.html. See also the preprint "Lyndon basis and the multiplicative formula for R-matrices", 2002.

[Ufer 2004] S. Ufer, "PBW bases for a class of braided Hopf algebras", J. Algebra 280:1 (2004), 84-119. MR 2005g:16079 Zbl 1113.16044 
Communicated by Susan Montgomery

Received 2008-04-03

Revised 2008-10-02

Accepted 2008-11-11

angiono@mate.uncor.edu

Universidad Nacional of Córdoba, Facultad of Matemática, Astronomía y Física, Medina Allende s/n, Ciudad Universitaria, 5000 Córdoba, Argentina 


\title{
Frobenius splittings of toric varieties
}

\author{
Sam Payne
}

\begin{abstract}
We discuss a characteristic free version of Frobenius splittings for toric varieties and give a polyhedral criterion for a toric variety to be diagonally split. We apply this criterion to show that section rings of nef line bundles on diagonally split toric varieties are normally presented and Koszul, and that Schubert varieties are not diagonally split in general.
\end{abstract}

\section{Introduction}

Fix an integer $q$ greater than one. Let $T=\operatorname{Spec} \mathbb{Z}[M]$ be the torus with character lattice $M$, and let $N$ be the dual lattice. Let $\Sigma$ be a complete fan in $N_{\mathbb{R}}$, with $X=X(\Sigma)$ the associated toric variety over $\mathbb{Z}$. Multiplication by $q$ preserves the fan and maps the lattice $N$ into itself, and therefore gives an endomorphism

$$
F: X \rightarrow X \text {. }
$$

Each $T$-orbit in $X$ is a torus that is preserved by $F$, which acts by taking a point $t$ to $t^{q}$. For example, if $X$ is projective space, then $F$ is given in homogeneous coordinates by

$$
\left[x_{0}: \cdots: x_{n}\right] \mapsto\left[x_{0}^{q}: \cdots: x_{n}^{q}\right] .
$$

If $q$ is prime and $k$ is the field with $q$ elements, then the restriction of $F$ to the variety $X_{k}$ is the absolute Frobenius morphism. Pulling back functions by $F$ gives a natural inclusion of $\mathrm{O}_{X}$-algebras $F^{*}: \mathrm{O}_{X} \hookrightarrow F_{*} \mathrm{O}_{X}$.

Definition 1.1. A splitting of $X$ is an $\mathrm{O}_{X}$-module map $\pi: F_{*} \mathrm{O}_{X} \rightarrow \mathrm{O}_{X}$ such that the composition $\pi \circ F^{*}$ is the identity on $O_{X}$.

Standard results from the theory of Frobenius splittings generalize in a straightforward way to these splittings of toric varieties. See Section 2 for details.

If $Y$ is a subvariety of $X$ cut out by an ideal sheaf $I_{Y}$ and $\pi\left(F_{*} I_{Y}\right)$ is contained in $I_{Y}$ then we say that $\pi$ is compatible with $Y$. If $Y$ is a toric variety embedded equivariantly in $X$, the closure of a subtorus of an orbit in $X$, then a splitting compatible with $Y$ induces a splitting of $Y$. We say that $X$ is diagonally split if

MSC2000: primary 14M25; secondary 13A35, 14M15, $16 \mathrm{~S} 37$.

Keywords: Frobenius splitting, toric variety, diagonal splitting, Koszul.

Supported by the Clay Mathematics Institute. 
there is a splitting of $X \times X$ that is compatible with the diagonal, for some $q$. Such splittings are of particular interest; by classic arguments of Mehta, Ramanan and Ramanathan, if $X$ is diagonally split then every ample line bundle on $X$ is very ample and defines a projectively normal embedding.

Our main result is a polyhedral criterion for a toric variety to be diagonally split. Let $v_{\rho}$ denote the primitive generator of a ray, or one-dimensional cone, in $\Sigma$. Let $M_{\mathbb{R}}=M \otimes_{\mathbb{Z}} \mathbb{R}$, and let the diagonal splitting polytope $\mathbb{F}_{X}$ be defined by

$$
\mathbb{F}_{X}=\left\{u \in M_{\mathbb{R}} \mid-1 \leq\left\langle u, v_{\rho}\right\rangle \leq 1 \text { for all } \rho \in \Sigma\right\} .
$$

We write $\frac{1}{q} M$ for the subgroup of $M_{\mathbb{R}}$ consisting of fractional lattice points $u$ such that $q u$ is in $M$.

Theorem 1.2. The toric variety $X$ is diagonally split if and only if the interior of $\mathbb{F}_{X}$ contains representatives of every equivalence class in $\frac{1}{q} M / M$.

While the existence of a compatible splitting of the diagonal in $X \times X$ implies that section rings of ample line bundles on $X$ are generated in degree one, compatible splittings of large semidiagonals in products of multiple copies of $X$ give further information on these section rings. For example, if the union of $\Delta \times X$ and $X \times \Delta$ is compatibly split in $X \times X \times X$, where $\Delta$ is the diagonal in $X \times X$, then it follows from standard arguments that the section ring of each ample line bundle on $X$ is normally presented, that is, generated in degree one with relations generated in degree two. For fixed $n$ greater than one, let $\Delta_{i}$ be the large semidiagonal

$$
\Delta_{i}=X^{i-1} \times \Delta \times X^{n-i-1},
$$

for $1 \leq i<n$.

Theorem 1.3. Let $X$ be a diagonally split toric variety. Then $\Delta_{1} \cup \cdots \cup \Delta_{n-1}$ is compatibly split in $X^{n}$.

In particular, if $X$ is diagonally split then the union of $\Delta \times X$ and $X \times \Delta$ is compatibly split in $X \times X \times X$, so the section ring of any ample line bundle on $X$ is normally presented. Analogous results hold for any finite collection of nef line bundles on $X$, as we now discuss.

For line bundles $L_{1}, \ldots, L_{r}$ on $X$, let $R\left(L_{1}, \ldots, L_{r}\right)$ be the section ring

$$
R\left(L_{1}, \ldots, L_{r}\right)=\bigoplus_{\left(\alpha_{1}, \ldots, \alpha_{r}\right) \in \mathbb{N}^{r}} H^{0}\left(X, L_{1}^{\alpha_{1}} \otimes \cdots \otimes L_{r}^{\alpha_{r}}\right) .
$$

We consider $R\left(L_{1}, \ldots, L_{r}\right)$ as a graded ring, where the degree of

$$
H^{0}\left(X, L_{1}^{\alpha_{1}} \otimes \cdots \otimes L_{r}^{\alpha_{r}}\right)
$$

is $\alpha_{1}+\cdots+\alpha_{r}$. In particular, the degree zero part of $R\left(L_{1}, \ldots, L_{r}\right)$ is $\mathbb{Z}$. Recall that a graded ring $R$ is Koszul if the ideal generated by elements of positive degree 
has a linear resolution as an $R$-module. See [Polishchuk and Positselski 2005] for background on Koszul rings and further details.

Theorem 1.4. Let $X$ be a complete, diagonally split toric variety, and let

$$
L_{1}, \ldots, L_{r}
$$

be nef line bundles on $X$. Then the section ring $R\left(L_{1}, \ldots, L_{r}\right)$ is normally presented and Koszul.

In particular, if $X$ is diagonally split then the section ring of any ample line bundle on $X$ is normally presented and Koszul. Well-known open problems ask whether every ample line bundle on a smooth projective toric variety gives a projectively normal embedding [Oda 1997] and, if so, whether its section ring is normally presented [Sturmfels 1996, Conjecture 13.19]. When the section ring is normally presented, it is natural to ask whether it is also Koszul. Addressing these questions and their analogues for singular toric varieties in as many cases as possible is one of the main motivations behind this work.

Remark 1.5. The section ring $R\left(L_{1}, \ldots, L_{r}\right)$ associated to a finite collection of line bundles is canonically identified with the section ring of the line bundle $O(1)$ on the projectivized vector bundle $\mathbb{P}\left(L_{1} \oplus \cdots \oplus L_{r}\right)$, which is also a toric variety. If $L_{1}, \ldots, L_{r}$ are nef and correspond to polytopes $P_{1}, \ldots, P_{r}$, then the Cayley sum is the polytope associated to $O(1)$. Cayley sums have also appeared prominently in recent work related to boundedness questions in toric mirror symmetry [Batyrev and Nill 2008; 2007; Haase et al. 2008].

Remark 1.6. Frobenius morphisms and their lifts to characteristic zero have been used powerfully in several other contexts related to the geometry of toric varieties, including by Buch, Lauritzen, Mehta and Thomsen [1997] to prove Bott vanishing and degeneration of the Hodge to de Rham spectral sequence, by Totaro [199?] to give a splitting of the weight filtration on Borel-Moore homology, by Smith [2000] to prove global $F$-regularity, by Brylinski and Zhang [2003] to prove degeneration of a spectral sequence computing equivariant cohomology with rational coefficients, and by Fujino [2007] to prove vanishing theorems for vector bundles and reflexive sheaves. Frobenius splittings have also played a role in unsuccessful attempts to show that section rings of ample line bundles on smooth toric varieties are normally presented [Bøgvad 1995]. We hope that this work will help revive the insight of Bøgvad and others into the potential usefulness of Frobenius splittings as a tool for understanding ample line bundles on toric varieties.

We conclude the introduction with an example illustrating Theorem 1.2 for Hirzebruch surfaces. As mentioned earlier, the proofs that section rings of ample line bundles on Schubert varieties are normally presented and Koszul via Frobenius 
splittings involved compatible splittings of semidiagonals in $(G / B)^{n}$. It has been an open question for over twenty years whether Schubert varieties themselves are diagonally split (see [Ramanathan 1987, Remark 3.6] and [Brion and Kumar 2005, p. 81]). The following example gives a negative answer: the Hirzebruch surface $F_{3}$ is a Schubert variety in the $G_{2}$-flag variety, and $F_{3}$ is not diagonally split.

Remark 1.7. To see that $F_{3}$ occurs as a Schubert variety in the $G_{2}$-flag variety, first note that for any $G, G / B$ is a $\mathbb{P}^{1}$-bundle over $G / P$, where $P$ is a minimal parabolic subgroup. If $w=s_{1} s_{2}$ is an element of length two in the Weyl group of $G$, and $P$ is the minimal parabolic corresponding to $s_{1}$, then $X_{w}$ is a $\mathbb{P}^{1}$-bundle over its image, which is a rational curve in $G / P$. In particular, $X_{w}$ is a Hirzebruch surface. Then $X_{s_{1}}$ is a rational curve in $X_{w}$ with self-intersection $\left\langle\alpha_{2}, \alpha_{1}^{\vee}\right\rangle$, where $\alpha_{i}$ is the simple root corresponding to $s_{i}$, and $\alpha_{i}^{\vee}=2 \alpha_{i} /\left\langle\alpha_{i}, \alpha_{i}\right\rangle$. See [Kempf 1976, Section 2] for details. For $G_{2}$, we can choose coordinates identifying the root lattice with the sublattice of $\mathbb{Z}^{3}$ consisting of those $\left(a_{1}, a_{2}, a_{3}\right)$ such that $a_{1}+a_{2}+a_{3}=0$, with simple roots $\alpha_{1}=(1,-1,0)$ and $\alpha_{2}=(-1,2,-1)$. Then $X_{s_{1}}$ is a curve of self-intersection -3 in $X_{w}$, and hence $X_{w}$ is isomorphic to $F_{3}$. See also [Anderson 2007] for a detailed study of the $G_{2}$-flag variety and its Schubert varieties.

Example 1.8. Let $a$ be a nonnegative integer, and let $\Sigma$ be the complete fan in $\mathbb{R}^{2}$ whose rays are spanned by $(1,0),(0,1),(0,-1)$, and $(-1, a)$. Then $X(\Sigma)$ is isomorphic to the Hirzebruch surface $F_{a}$, the projectivization of the vector bundle $\mathcal{O}_{\mathbb{P}^{1}} \oplus \mathcal{O}_{\mathbb{P}^{1}}(a)$ [Fulton 1993, pp. 7-8]. Let $q \geq 2$ be an integer. By Theorem 1.2, $X$ is diagonally split if and only if the fractional lattice points in the interior of $\mathbb{F}_{X}$ represent every equivalence class in $\frac{1}{q} \mathbb{Z}^{2} / \mathbb{Z}^{2}$. The polytopes $\mathbb{F}_{X}$ for different values of $a$ are shown below.

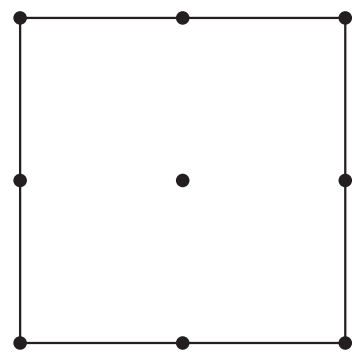

$a=0$

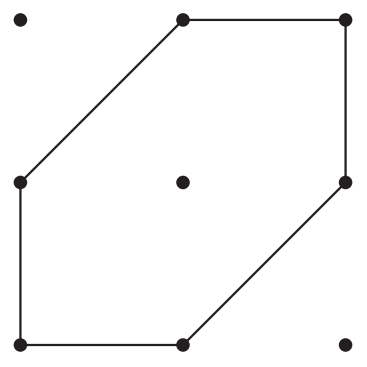

$a=1$

If $a$ is equal to 0 or 1 , the interior of $\mathbb{F}_{X}$ contains the half open unit square $[0,1) \times[0,1)$, which contains representatives of every equivalence class in $\frac{1}{q} \mathbb{Z}^{2} / \mathbb{Z}^{2}$. Therefore, $F_{0}$ and $F_{1}$ are diagonally split for all $q$.

If $a=2$, then $\mathbb{F}_{X}$ is the parallelogram with vertices $( \pm 1,0), \pm(1,1)$. For $0 \leq m<q$, the interior of this intersection contains the fractional lattice points 
$(m / q, n / q)$ for $(m-q) / 2<n<(m+q) / 2$. If $q$ is odd, then these represent every equivalence class in $\frac{1}{q} \mathbb{Z}^{2} / \mathbb{Z}^{2}$. In particular, $F_{2}$ is diagonally split for $q$ odd.

If $a$ is greater than two, then $\mathbb{F}_{X}$ is the parallelogram with vertices $( \pm 1,0)$, $\pm(1,2 / a)$. The only points in the interior of this intersection whose first coordinate is integral, are of the form $(0, y)$ for $-1 / a<y<1 / a$. In particular, the equivalence class of $(0,\lfloor q / 2\rfloor / q)$ in $\frac{1}{q} \mathbb{Z}^{2} / \mathbb{Z}^{2}$ is not represented by any point in the interior of this intersection. Therefore, $F_{a}$ is not diagonally split for $a$ greater than two.
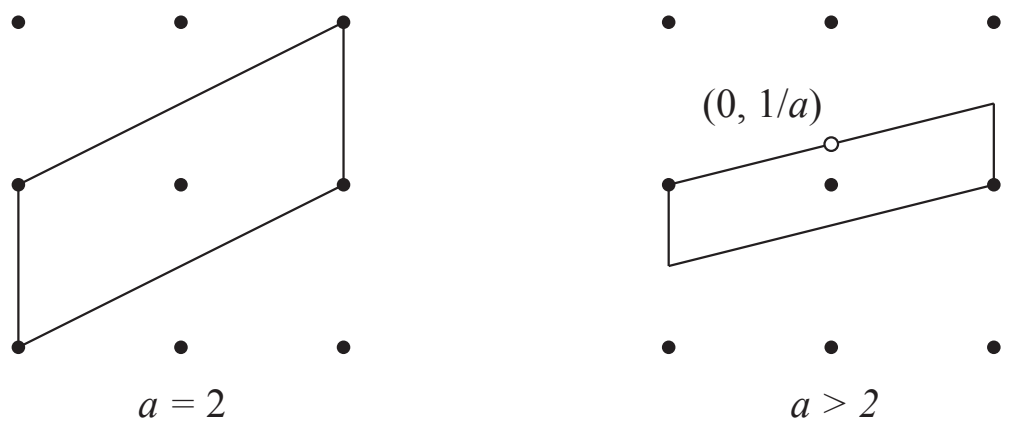

\section{Preliminaries}

Frobenius splittings were introduced and developed by Mehta, Ramanathan, and their collaborators in the 1980s. The original paper of Mehta and Ramanathan is exceedingly well written and remains an excellent first introduction to the subject [1985]. Frobenius splittings were rapidly applied to give elegant unified proofs that all ample line bundles on generalized Schubert varieties of all types are very ample and give projectively normal embeddings whose images are cut out by quadrics [Ramanan and Ramanathan 1985; Ramanathan 1987]. Inamdar and Mehta [1994], and independently Bezrukavnikov [1995], later showed that the homogeneous coordinate rings of these embeddings are Koszul. In characteristic zero, these results are deduced from the positive characteristic case using general semicontinuity theorems. See the recent book of Brion and Kumar [2005] for a unified exposition of these results, along with further details, references, and applications. On toric varieties, the Frobenius endomorphisms lift to endomorphisms over $\mathbb{Z}$, and it seems easiest and most natural to work independently of the characteristic using these lifted endomorphisms. One feature of this approach is that we can prove results about section rings of toric varieties over $\mathbb{Z}$, or an arbitrary field, by producing a splitting of the diagonal in $X \times X$ for a single $q$.

We begin by considering the structure of $F_{*} \mathrm{O}_{X}$ as an $\mathrm{O}_{X}$-module. As a sheaf of groups, $F_{*} \mathrm{O}_{X}$ evaluated on the invariant affine open set $U_{\sigma}$ associated to a cone $\sigma \in \Sigma$ is the coordinate ring $\mathbb{Z}\left[U_{\sigma}\right]$, which is usually identified with the semigroup ring $\mathbb{Z}\left[\sigma^{\vee} \cap M\right]$. However, the module structure on $F_{*} \mathbb{Z}\left[U_{\sigma}\right]$ is different from the 
action of $\mathbb{Z}\left[U_{\sigma}\right]$ on itself. For this reason, we identify $F_{*} \mathbb{Z}\left[U_{\sigma}\right]$ with the semigroup ring of fractional lattice points

$$
F_{*} \mathbb{Z}\left[U_{\sigma}\right]=\mathbb{Z}\left[\sigma^{\vee} \cap \frac{1}{q} M\right],
$$

taking a monomial $x^{u} \in \mathbb{Z}\left[U_{\sigma}\right]$ to $x^{u / q}$. The action of $\mathbb{Z}\left[U_{\sigma}\right]$ on $F_{*} \mathbb{Z}\left[U_{\sigma}\right]$ is then induced by the natural action of $M$ on $\frac{1}{q} M$, so

$$
x^{u} \cdot x^{u^{\prime}}=x^{u+u^{\prime}}
$$

for $u \in M$ and $u^{\prime} \in \frac{1}{q} M$. If $Y$ is a toric variety embedded equivariantly in $X$, then a splitting $\pi$ is compatible with $Y$ if and only if the induced map

$$
\mathbb{Z}\left[U_{\sigma}\right] \stackrel{\sim}{\rightarrow} \mathbb{Z}\left[\sigma^{\vee} \cap \frac{1}{q} M\right] \stackrel{\pi}{\rightarrow} \mathbb{Z}\left[U_{\sigma}\right]
$$

maps $I_{Y}\left(U_{\sigma}\right)$ into $I_{Y}\left(U_{\sigma}\right)$ for every $\sigma \in \Sigma$.

We now summarize some basic properties of compatible splittings and their applications to section rings of ample line bundles. Let $X$ be a complete toric variety, and let $L$ be a line bundle on $X$. A splitting $\pi$ of $X$ makes $O_{X}$ a direct summand of $F_{*} \mathrm{O}_{X}$ and hence $L$ a direct summand of $L \otimes F_{*} \mathrm{O}_{X}$. By the projection formula, $L \otimes F_{*} \mathcal{O}_{X}$ is isomorphic to $F_{*}\left(F^{*} L\right)$, and we claim that $F^{*} L$ is isomorphic to $L^{q}$. To see this, note that there is a $T$-invariant Cartier divisor $D$ such that $L$ is isomorphic to $O(D)$ [Fulton 1993, Section 3.4]. The restriction of $D$ to an invariant affine open $U_{\sigma}$ is the divisor of a rational function $x^{u}$ for some $u \in M$, and hence the restriction of $F^{*} D$ to $U_{\sigma}$ is the divisor of $x^{q u}$. It follows that $F^{*} L$ is isomorphic to $L^{q}$, as claimed. Now, since cohomology commutes with direct sums, $\pi$ induces a split injection

$$
H^{i}(X, L) \hookrightarrow H^{i}\left(X, L^{q}\right),
$$

for every $i$. Iterating this argument gives split injections of $H^{i}(X, L)$ in $H^{i}\left(X, L^{q^{r}}\right)$ for all positive integers $r$. In particular, if $H^{i}\left(X, L^{q^{r}}\right)$ vanishes for some $r$, as is the case when $L$ is ample, then $H^{i}(X, L)$ vanishes as well.

The proofs of the following five propositions are essentially identical to the standard proofs of the analogous results for Frobenius splittings, and are omitted. See [Brion and Kumar 2005]; Proposition 1.2.1, Theorem 1.2.8, and Exercises 1.5.E.1, 1.5.E.2, and 1.5.E.3, respectively, for the case where the line bundles in question are ample. The extensions to nef bundles can be deduced following the arguments in [Inamdar 1994], using the fact that any nef line bundle on $X$ is the pullback of an ample line bundle on some toric variety $X^{\prime}$ under a proper birational toric morphism $f: X \rightarrow X^{\prime}$.

Proposition 2.1. Let $Y$ and $Y^{\prime}$ be toric varieties equivariantly embedded in $X$. If $Y \cup Y^{\prime}$ is split compatibly in $X$ then $Y, Y^{\prime}$, and $Y \cap Y^{\prime}$ are split compatibly in $X$. 
Proposition 2.2. Let $Y$ be a compatibly split subvariety of $X$. If $L$ is a nef line bundle on $X$ then the restriction map

$$
H^{0}(X, L) \rightarrow H^{0}(Y, L)
$$

is surjective and $H^{1}\left(X, I_{Y} \otimes L\right)=0$.

Proposition 2.3. Let $L_{1}, \ldots, L_{r}$ be nef line bundles on $X$. If the diagonal is compatibly split in $X \times X$ then the section ring $R\left(L_{1}, \ldots, L_{r}\right)$ is normally generated.

Proposition 2.4. Let $L_{1}, \ldots, L_{r}$ be nef line bundles on $X$. If the union of $\Delta \times X$ and $X \times \Delta$ is compatibly split in $X \times X \times X$ then the section ring $R\left(L_{1}, \ldots, L_{r}\right)$ is normally presented.

Proposition 2.5. Let $L_{1}, \ldots, L_{r}$ be nef line bundles on $X$. If $\Delta_{1} \cup \cdots \cup \Delta_{n-1}$ is compatibly split in $X^{n}$ for every $n$ then the section ring $R\left(L_{1}, \ldots, L_{r}\right)$ is normally presented and Koszul.

\section{Canonical splittings}

Every toric variety has a splitting, and among all splittings of $X$ there is a unique one that extends to every toric compactification $X^{\prime} \supset X$ and lifts to every proper birational toric modification $X^{\prime \prime} \rightarrow X^{\prime}$ of such a compactification. If $q$ is prime and $k$ is the field with $q$ elements, then the restriction of this splitting to $X_{k}$ is the unique Frobenius splitting that is canonical in the sense of Mathieu [Brion and Kumar 2005, Chapter 4]. We now describe this canonical splitting, starting with its restriction to the dense torus $T$.

Let $\pi_{0}$ be the map of $\mathbb{Z}[T]$-modules from $F_{*} \mathbb{Z}[T]$ to $\mathbb{Z}[T]$ given by

$$
\pi_{0}\left(x^{u}\right)= \begin{cases}x^{u} & \text { if } u \in M \\ 0 & \text { otherwise }\end{cases}
$$

The pullback map $F^{*}: \mathbb{Z}[T] \rightarrow F_{*} \mathbb{Z}[T]$ is induced by the inclusion of $M$ in $\frac{1}{q} M$; if $q$ is prime and $k$ is the field with $q$ elements, then the induced map $k[T] \rightarrow F_{*} k[T]$ may be identified with the inclusion of $k[T]$ in $k[T]^{1 / q}$.

In particular, $\pi_{0} \circ F_{*}$ is the identity, and hence gives a splitting of $T$.

Proposition 3.1. For any toric variety $X, \pi_{0}$ extends to a splitting of $X$.

Proof. The composition $\pi_{0} \circ F^{*}$ is the identity, and for each affine open $U_{\sigma}, \pi_{0}$ maps $\mathbb{Z}\left[\sigma^{\vee} \cap \frac{1}{q} M\right]$ into $\mathbb{Z}\left[\sigma^{\vee} \cap M\right]$.

Properties of this canonical splitting $\pi_{0}$ are closely related to Smith's proof [2000, Proposition 6.3] that toric varieties are globally $F$-regular.

Proposition 3.2. The canonical splitting $\pi_{0}$ is compatible with every $T$-invariant subvariety. 
Proof. First we claim that $\pi_{0}$ is compatible with the union of the $T$-invariant divisors. To see this, note that the ideal sheaf $I$ of the union of the invariant divisors is given by

$$
I\left(U_{\sigma}\right)=\mathbb{Z}\left[\operatorname{int}\left(\sigma^{\vee}\right) \cap M\right],
$$

where $\operatorname{int}\left(\sigma^{\vee}\right)$ is the interior of $\sigma^{\vee}$. If $u$ is a fractional lattice point in the interior of $\sigma^{\vee}$ then $\pi_{0}\left(x^{u}\right)$ is either zero or $x^{u}$, and so is contained in $I\left(U_{\sigma}\right)$, which proves the claim. The proposition then follows from Proposition 2.1, since every $T$-invariant subvariety is an intersection of invariant divisors.

However, if $X$ is positive dimensional then the canonical splitting $\pi_{0}$ of $X \times X$ is not compatible with the diagonal $\Delta$. To see this, observe that if $u \in \frac{1}{q} M$ is not in $M$, then $1-x^{u} \otimes x^{-u}$ is in $F_{*} I_{\Delta}$, but

$$
\pi_{0}\left(1-x^{u} \otimes x^{-u}\right)=1,
$$

which is not in $I_{\Delta}$. To apply the standard techniques relating splittings to section rings of ample line bundles discussed in Section 2, we must look for other splittings of $X \times X$, and $X^{r}$ for $r$ greater than two, that are compatible with the diagonal and the union of the large semidiagonals, respectively.

\section{Splittings of diagonals}

We now describe the space of all splittings of a toric variety and use this description to characterize diagonally split toric varieties. First, it is helpful to consider the structure of $F_{*} \mathrm{O}_{X}$ as an $\mathrm{O}_{X}$-module in more detail.

Recall that an equivariant structure, or $T$-linearization, on a coherent sheaf $\mathscr{F}_{F}$ on $X$ is an isomorphism of sheaves on $T \times X$,

$$
\varphi: \mu^{*} \mathscr{F} \rightarrow p^{* \mathscr{F}},
$$

where $\mu: T \times X \rightarrow X$ is the torus action and $p$ is the second projection, that satisfies the usual cocycle condition [Brion and Kumar 2005, Section 2.1]. For example, the natural equivariant structure on $\mathrm{O}_{X}$ is given by $1 \otimes x^{u} \mapsto x^{-u} \otimes x^{u}$. In general, the push forward of an equivariant sheaf under an equivariant morphism does not carry a natural equivariant structure. However, the equivariant endomorphism $F$ has the property that $F_{*} O_{X}$ is equivariantizable [Bøgvad 1998; Thomsen 2000]; it is possible to choose an equivariant structure as follows. First, choose representatives $u_{1}, \ldots, u_{s}$ of the cosets in $\frac{1}{q} M / M$. Let $\varphi$ be the map from $\mu^{*} F_{*} \mathrm{O}_{X}$ to $p^{*} F_{*} \mathcal{O}_{X}$ that takes $1 \otimes x^{u}$ to $x^{u_{i}-u} \otimes x^{u}$, for $u$ in the coset $u_{i}+M$. It is straightforward to check that $\varphi$ is an isomorphism and gives an equivariant structure on $F_{*} \mathrm{O}_{X}$, as required.

A splitting of $X$ restricts to a splitting of $T$, and two splittings of $X$ agree if and only if they agree on $T$, so we describe the space of all splittings of $X$ in terms of 
splittings of $T$ that extend to $X$, as follows. For fractional lattice points $a \in \frac{1}{q} M$, let

$$
\pi_{a}: F_{*} \mathbb{Z}[T] \rightarrow \mathbb{Z}[T]
$$

be the map given by

$$
\pi_{a}\left(x^{u}\right)= \begin{cases}x^{a+u} & \text { if } a+u \text { is in } M \\ 0 & \text { otherwise. }\end{cases}
$$

Lemma 4.1. The set of maps $\pi_{a}$, for a in $\frac{1}{q} M$, is a $\mathbb{Z}$-basis for

$$
\operatorname{Hom}\left(F_{*} \mathbb{Z}[T], \mathbb{Z}[T]\right) .
$$

Proof. The maps $\pi_{a}$ are independent, and the free generators $x^{u_{1}}, \ldots, x^{u_{s}}$ for $F_{*} \mathbb{Z}[T]$ can be sent to an arbitrary $s$-tuple of elements of $\mathbb{Z}[T]$ by a suitable linear combination of the maps $\pi_{a}$.

If we choose an equivariant structure for $\operatorname{Hom}\left(F_{*} \mathbb{Z}[T], \mathbb{Z}[T]\right)$, as above, then the maps $\pi_{a}$ form a $T$-eigenbasis. Therefore, a rational section

$$
\pi=c_{1} \pi_{a_{1}}+\cdots+c_{r} \pi_{a_{r}}
$$

of the sheaf $\mathscr{H o m}\left(F_{*} \mathrm{O}_{X}, \mathrm{O}_{X}\right)$, with each $c_{i}$ nonzero, extends to $X$ if and only if each $\pi_{a_{i}}$ is regular on $X$. For a ray, or one-dimensional cone $\rho$ in $\Sigma$, we write $v_{\rho}$ for the primitive generator of $\rho$.

Proposition 4.2. Let $U_{\sigma}$ be an affine toric variety. Then $\pi_{a}$ is regular on $U_{\sigma}$ if and only if $\left\langle a, v_{\rho}\right\rangle$ is greater than minus one for each ray $\rho$ in $\sigma$.

Proof. The map $\pi_{a}$ is regular on $U_{\sigma}$ if and only if it takes

$$
\mathbb{Z}\left[\sigma^{\vee} \cap \frac{1}{q} M\right]
$$

into $\mathbb{Z}\left[\sigma^{\vee} \cap M\right]$. Suppose $\left\langle a, v_{\rho}\right\rangle$ is greater than minus one for each ray $\rho$ in $\sigma$ and $u$ is in $\sigma^{\vee} \cap \frac{1}{q} M$. Either $\pi_{a}\left(x^{u}\right)$ is zero or $a+u$ is in $M$ and $\left\langle u, v_{\rho}\right\rangle$ is a nonnegative integer for all rays $\rho$ in $\sigma$, and hence $a+u$ is in $\sigma^{\vee}$. Therefore $\pi_{a}$ extends to $U_{\sigma}$. Conversely, if $\left\langle a, v_{\rho}\right\rangle$ is less than or equal to minus one for some $\rho$, then it is straightforward to produce points $u \in \sigma^{\vee}$ such that $a+u$ is in $M$, but not in $\sigma^{\vee}$. In this case, $\pi_{a}$ is not regular on $U_{\sigma}$.

We follow the usual toric convention fixing $K=-\sum D_{\rho}$, the sum of the prime $T$-invariant divisors each with multiplicity minus one, as a convenient representative of the canonical class. The polytope associated to a divisor $D=\sum d_{\rho} D_{\rho}$ is

$$
P_{D}=\left\{u \in M_{\mathbb{B}} \mid\left\langle u, v_{\rho}\right\rangle \geq-d_{\rho} \text { for all } \rho\right\} .
$$

In particular, the polytope $P_{-K}$ associated to the anticanonical divisor is

$$
P_{-K}=\left\{u \in M_{\mathbb{R}} \mid\left\langle u, v_{\rho}\right\rangle \geq-1 \text { for all } \rho\right\} .
$$


The interior of the polytope $P_{-K}$ controls the space of $\mathrm{O}_{X}$-module maps from $F_{*} \mathcal{O}_{X}$ to $\mathscr{O}_{X}$ as follows.

Proposition 4.3. The set of maps $\pi_{a}$ for fractional lattice points $a$ in the interior of $P_{-K}$ is a basis for $\operatorname{Hom}\left(F_{*} \mathrm{O}_{X}, \mathrm{O}_{X}\right)$.

Proof. If $a$ is not in the interior of $P_{-K}$ then $\left\langle a, v_{\rho}\right\rangle$ is less than or equal to minus one for some ray $\rho \in \Sigma$ and then $\pi_{a}$ is not regular on $U_{\rho}$. Conversely, if $a$ is in the interior of $P_{-K}$, then $\pi_{a}$ extends to every invariant affine open subvariety of $X$, by Proposition 4.2, and therefore is regular on $X$.

Remark 4.4. When $X$ is smooth, Proposition 4.3 corresponds to the natural identification between $\operatorname{Hom}\left(F_{*} \mathrm{O}_{X}, \mathrm{O}_{X}\right)$ and $H^{0}\left(X, K_{X}^{1-q}\right)$ given by duality for finite flat morphisms [Brion and Kumar 2005, Section 1.3].

Proposition 4.5. A map $\sum c_{a} \pi_{a}$ in $\operatorname{Hom}\left(F_{*} O_{X}, O_{X}\right)$ is a splitting if and only if $c_{0}=1$.

Proof. Zero is the only lattice point in the interior of $P_{-K} \cap M$, so the image of $x^{u}$ under $\pi=\sum c_{a} \pi_{a}$ is equal to $c_{0} x^{u}$ for $u \in M$. In particular, since $F^{*}$ maps $x^{u}$ to $x^{u}$ in $F_{*} O_{X}, \pi \circ F^{*}$ is the identity if and only if $c_{0}$ is equal to one.

The set of splittings of $X$ is an affine hyperplane in $\operatorname{Hom}\left(F_{*} \mathrm{O}_{X}, \mathrm{O}_{X}\right)$ by Proposition 4.5. For any subvariety $Y \subset X$, the condition that $\pi\left(F_{*} I_{Y}\right)$ is contained in $I_{Y}$ cuts out a linear subspace of $\operatorname{Hom}\left(F_{*} \mathrm{O}_{X}, \mathrm{O}_{X}\right)$. So the set of splittings of $X$ that are compatible with $Y$ is an affine subspace of $\operatorname{Hom}\left(F_{*} \mathrm{O}_{X}, \mathrm{O}_{X}\right)$, which may be empty. We now prove Theorem 1.2, which gives a necessary and sufficient condition for the space of splittings of $X \times X$ that are compatible with the diagonal to be nonempty.

Proof of Theorem 1.2. Suppose

$$
\pi=\sum c_{a, a^{\prime}} \pi_{a, a^{\prime}}
$$

is a splitting of $X \times X$ that is compatible with the diagonal. Then the restriction of $\pi$ to the dense torus is a splitting compatible with the diagonal in $T \times T$. For any $u \in \frac{1}{q} M$, we have

$$
1-x^{u} \otimes x^{-u}
$$

in $F_{*} I_{\Delta}$, where $I_{\Delta}$ is the ideal of the diagonal in $T \times T$. Since $\pi$ (1) is equal to one, the restriction of $\pi\left(x^{u} \otimes x^{-u}\right)$ to the diagonal must also be equal to one. Now the restriction of $\pi\left(x^{u} \otimes x^{-u}\right)$ to the diagonal is a Laurent polynomial in $\mathbb{Z}[T]$ whose constant term is

$$
\sum_{a \in[u]} c_{-a, a}
$$

where $[u]$ is the coset of $u$ in $\frac{1}{q} M / M$. Since the polytope associated to $-K_{X \times X}$ is $P_{-K_{X}} \times P_{-K_{X}}$, there must be a representative $a$ of $[u]$ such that both $a$ and $-a$ are 
contained in the interior of $P_{-K_{X}}$, which means that $a$ is contained in the interior of the diagonal splitting polytope

$$
\mathbb{F}_{X}=P_{-K_{X}} \cap-P_{-K_{X}} .
$$

For the converse, suppose that every nonzero equivalence class $\left[u_{i}\right]$ in $\frac{1}{q} M / M$ has a representative $a_{i}$ in the interior of $\mathbb{F}_{X}$. Then

$$
\pi_{\Delta}=\pi_{0}+\sum_{i=1}^{s} \pi_{a_{i},-a_{i}}
$$

is a splitting, and we claim that $\pi_{\Delta}$ is compatible with the diagonal. To see this, note that the ideal of the diagonal in $U_{\sigma} \times U_{\sigma}$ is generated by the Laurent polynomials $1-x^{u} \otimes x^{-u}$ for $u$ in $\sigma^{\vee} \cap M$. Then $F_{*} I_{\Delta}$ is generated as a $\mathbb{Z}\left[U_{\sigma} \times U_{\sigma}\right]$-module by the

$$
x^{b}-x^{b} \cdot\left(x^{u} \otimes x^{-u}\right),
$$

as $b=\left(b_{1}, b_{2}\right)$ ranges over $\frac{1}{q}(M \times M)$ and $u$ ranges over $\frac{1}{q} M$. Now the restriction of $\pi_{\Delta}\left(x^{b}\right)$ to the diagonal is $x^{b_{1}+b_{2}}$ if $b_{1}+b_{2}$ is in $M$ and zero otherwise. In particular, the restriction of $\pi_{\Delta}\left(x^{b}-x^{b} \cdot\left(x^{u} \otimes x^{-u}\right)\right)$ to the diagonal vanishes, as required.

Proof of Theorem 1.3. Suppose the diagonal is compatibly split in $X \times X$. Then every nonzero equivalence class $\left[u_{j}\right]$ in $\frac{1}{q} M / M$ is represented by a fractional lattice point $a_{j}$ in the interior of $\mathbb{F}_{X}$, by Theorem 1.2.

A splitting of $X^{n}$ is compatible with the union $\Delta_{1} \cup \cdots \cup \Delta_{n-1}$ if it is compatible with each $\Delta_{i}$. For $u \in M$, let $u^{(i)}$ denote the lattice point in $M^{n}$ whose only nonzero coordinate is the $i$-th one, which is equal to $u$. The ideal of $\Delta_{i}$ is generated by the functions

$$
1-x^{u^{(i)}} \cdot x^{-u^{(i+1)}}
$$

We claim that the splitting

$$
\pi=\pi_{0}+\sum_{i=1}^{n-1} \sum_{j} \pi_{a_{j}^{(i)}-a_{j}^{(i+1)}}
$$

is compatible with $\Delta_{i}$ for $1 \leq i<n$, and hence with the union $\Delta_{1} \cup \cdots \cup \Delta_{n-1}$. The proof of the claim is then similar to the proof of Theorem 1.2 above, and the theorem follows.

Proof of Theorem 1.4. Suppose the diagonal is compatibly split in $X \times X$. Then $\Delta_{1} \cup \cdots \cup \Delta_{n-1}$ is compatibly split in $X^{n}$ for every $n$, by Theorem 1.3. Therefore, for any nef line bundles $L_{1}, \ldots, L_{r}$ on $X$ the section ring $R\left(L_{1}, \ldots, L_{r}\right)$ is normal and Koszul, by Proposition 2.5. 
Remark 4.6. Pairs of opposite lattice points $u$ and $-u$ in the polytopes associated to anticanonical divisors have also appeared in relation to the classification of smooth toric Fano varieties. In particular, Ewald [1988] conjectured twenty years ago that if $X$ is a smooth toric Fano variety then $\mathbb{F}_{X}$ contains a basis for the character lattice $M$. Ewald's conjecture has been verified for smooth toric Fano varieties of dimension less than or equal to seven by Øbro [2007, Section 4.1]. However, it remains unknown in higher dimensions whether there exists a single nonzero lattice point $u$ in $\mathbb{F}_{X}$ [Kreuzer and Nill 2007, Section 4.6].

\section{Acknowledgments}

This work grew out of an expository talk at a mini-workshop on normality of lattice polytopes at Oberwolfach. I thank the organizers, C. Haase, T. Hibi, and D. Maclagan, as well as the other participants, for the chance to be part of such a stimulating event, and the MFO for their hospitality. While preparing this paper, I benefited from helpful discussions with D. Anderson, D. Eisenbud, N. Fakhruddin, B. Howard, and A. Paffenholz, and from the referee's thoughtful comments. Finally, I am grateful to N. Lauritzen and J. Thomsen for pointing out that the methods in a first version of this paper can be used to give the example of the nondiagonally split Schubert variety presented here.

\section{References}

[Anderson 2007] D. Anderson, "Chern class formulas for $G_{2}$ Schubert loci”, preprint, 2007. arXiv 0712.2641

[Batyrev and Nill 2007] V. Batyrev and B. Nill, "Multiples of lattice polytopes without interior lattice points", Mosc. Math. J. 7:2 (2007), 195-207, 349. MR 2008g:52018 Zbl 1134.52020

[Batyrev and Nill 2008] V. Batyrev and B. Nill, "Combinatorial aspects of mirror symmetry", pp. 35-66 in Integer points in polyhedra-geometry, number theory, representation theory, algebra, optimization, statistics (Snowbird, UT, 2006), edited by M. Beck et al., Contemp. Math. 452, Amer. Math. Soc., Providence, RI, 2008. MR 2405763 arXiv math/0703456

[Bezrukavnikov 1995] R. Bezrukavnikov, "Koszul property and Frobenius splitting of Schubert varieties", preprint, 1995. arXiv alg-geom/9502021

[Bøgvad 1995] R. Bøgvad, "On the homogeneous ideal of a projective nonsingular toric variety", preprint, 1995. arXiv alg-geom/9501012

[Bøgvad 1998] R. Bøgvad, "Splitting of the direct image of sheaves under the Frobenius", Proc. Amer. Math. Soc. 126:12 (1998), 3447-3454. MR 99h:14051 Zbl 0902.14038

[Brion and Kumar 2005] M. Brion and S. Kumar, Frobenius splitting methods in geometry and representation theory, Progress in Mathematics 231, Birkhäuser, Boston, 2005. MR 2005k:14104 Zbl 1072.14066

[Brylinski and Zhang 2003] J.-L. Brylinski and B. Zhang, "Equivariant Todd classes for toric varieties", preprint, 2003. arXiv math/0311318

[Buch et al. 1997] A. Buch, J. F. Thomsen, N. Lauritzen, and V. Mehta, "The Frobenius morphism on a toric variety", Tohoku Math. J. (2) 49:3 (1997), 355-366. MR 98j:14020 Zbl 0899.14026 
[Ewald 1988] G. Ewald, "On the classification of toric Fano varieties", Discrete Comput. Geom. 3:1 (1988), 49-54. MR 88m:14037 Zbl 0686.14042

[Fujino 2007] O. Fujino, "Multiplication maps and vanishing theorems for toric varieties", Math. Z. 257:3 (2007), 631-641. MR 2008g:14092 Zbl 1129.14029

[Fulton 1993] W. Fulton, Introduction to toric varieties, Annals of Mathematics Studies 131, Princeton University Press, Princeton, NJ, 1993. MR 94g:14028 Zbl 0813.14039

[Haase et al. 2008] C. Haase, B. Nill, and S. Payne, "Cayley decompositions of lattice polytopes and upper bounds for $h^{*}$-polynomials", arXiv 0804.3667

[Inamdar 1994] S. P. Inamdar, "A note on Frobenius splitting of Schubert varieties and linear syzygies”, Amer. J. Math. 116:6 (1994), 1587-1590. MR 96a:14055 Zbl 0819.14020

[Inamdar and Mehta 1994] S. P. Inamdar and V. B. Mehta, "Frobenius splitting of Schubert varieties and linear syzygies", Amer. J. Math. 116:6 (1994), 1569-1586. MR 96a:14054 Zbl 0817.14032

[Kempf 1976] G. R. Kempf, "Linear systems on homogeneous spaces", Ann. of Math. (2) 103:3 (1976), 557-591. MR 53 \#13229 Zbl 0327.14016

[Kreuzer and Nill 2007] M. Kreuzer and B. Nill, "Classification of toric Fano 5-folds", preprint, 2007. arXiv math/0702890

[Mehta and Ramanathan 1985] V. B. Mehta and A. Ramanathan, "Frobenius splitting and cohomology vanishing for Schubert varieties", Ann. of Math. (2) 122:1 (1985), 27-40. MR 86k:14038 Zbl 0601.14043

[Øbro 2007] M. Øbro, Classification of smooth Fano polytopes, PhD Thesis, University of Århus, Århus, Denmark, 2007.

[Oda 1997] T. Oda, "Problems on Minkowski sums of convex lattice polytopes", preprint, 1997. arXiv 0812.1418

[Polishchuk and Positselski 2005] A. Polishchuk and L. Positselski, Quadratic algebras, University Lecture Series 37, American Mathematical Society, Providence, RI, 2005. MR 2006f:16043 Zbl 1145.16009

[Ramanan and Ramanathan 1985] S. Ramanan and A. Ramanathan, "Projective normality of flag varieties and Schubert varieties", Invent. Math. 79:2 (1985), 217-224. MR 86j:14051 Zbl 0553.14023

[Ramanathan 1987] A. Ramanathan, "Equations defining Schubert varieties and Frobenius splitting of diagonals", Inst. Hautes Études Sci. Publ. Math. 65 (1987), 61-90. MR 88k:14032 Zbl 0634. 14035

[Smith 2000] K. E. Smith, "Globally F-regular varieties: applications to vanishing theorems for quotients of Fano varieties”, Michigan Math. J. 48 (2000), 553-572. MR 2001k:13007 Zbl 0994.14012

[Sturmfels 1996] B. Sturmfels, Gröbner bases and convex polytopes, University Lecture Series 8, American Mathematical Society, Providence, RI, 1996. MR 97b:13034 Zbl 0856.13020

[Thomsen 2000] J. F. Thomsen, "Frobenius direct images of line bundles on toric varieties", $J$. Algebra 226:2 (2000), 865-874. MR 2001f:14095 Zbl 0957.14036

[Totaro 199?] B. Totaro, "Chow groups, Chow cohomology, and linear varieties", preprint, 199?, Available at http://www.dpmms.cam.ac.uk/ bt219/linear.dvi. To appear in J. Algebraic Geom.

Communicated by Vasudevan Srinivas

Received 2008-05-08

Revised 2008-10-15

Accepted 2008-11-22

spayne@stanford.edu

Department of Mathematics, Building 380, Sloan Hall, Stanford University, Stanford, CA 94305, United States 


\section{Guidelines for Authors}

Authors may submit manuscripts in PDF format on-line at the Submission page at the ANT website.

Originality. Submission of a manuscript acknowledges that the manuscript is original and and is not, in whole or in part, published or under consideration for publication elsewhere. It is understood also that the manuscript will not be submitted elsewhere while under consideration for publication in this journal.

Language. Articles in ANT are usually in English, but articles written in other languages are welcome.

Required items. A brief abstract of about 150 words or less must be included. It should be self-contained and not make any reference to the bibliography. If the article is not in English, two versions of the abstract must be included, one in the language of the article and one in English. Also required are keywords and subject classifications for the article, and, for each author, postal address, affiliation (if appropriate), and email address.

Format. Authors are encouraged to use $\mathrm{LT}_{\mathrm{E}} \mathrm{X}$ but submissions in other varieties of $\mathrm{T}_{\mathrm{E}} \mathrm{X}$, and exceptionally in other formats, are acceptable. Initial uploads should be in PDF format; after the refereeing process we will ask you to submit all source material.

References. Bibliographical references should be complete, including article titles and page ranges. All references in the bibliography should be cited in the text. The use of $\mathrm{BibT}_{\mathrm{E}} \mathrm{X}$ is preferred but not required. Tags will be converted to the house format, however, for submission you may use the format of your choice. Links will be provided to all literature with known web locations and authors are encouraged to provide their own links in addition to those supplied in the editorial process.

Figures. Figures must be of publication quality. After acceptance, you will need to submit the original source files in vector graphics format for all diagrams in your manuscript: vector EPS or vector PDF files are the most useful.

Most drawing and graphing packages (Mathematica, Adobe Illustrator, Corel Draw, MAT$\mathrm{LAB}$, etc.) allow the user to save files in one of these formats. Make sure that what you are saving is vector graphics and not a bitmap. If you need help, please write to graphics@mathscipub.org with details about how your graphics were generated.

White Space. Forced line breaks or page breaks should not be inserted in the document. There is no point in your trying to optimize line and page breaks in the original manuscript. The manuscript will be reformatted to use the journal's preferred fonts and layout.

Proofs. Page proofs will be made available to authors (or to the designated corresponding author) at a Web site in PDF format. Failure to acknowledge the receipt of proofs or to return corrections within the requested deadline may cause publication to be postponed. 


\section{Algebra \& Number Theory}

$\begin{array}{lll}\text { Volume } 3 & \text { No. } 1 \quad 2009\end{array}$

On the additive dilogarithm

SINAN ÜNVER

Nichols algebras with standard braiding

IVAN EZEQUIEL ANGIONO

Frobenius splittings of toric varieties

SAM PAYNE 